\title{
Impacts of government incentives to R\&D, innovation and productivity : a microeconometric analysis of the Québec case
}

Citation for published version (APA):

Baghana, R. S. A. (2013). Impacts of government incentives to $R \& D$, innovation and productivity : a microeconometric analysis of the Québec case. [Doctoral Thesis, Maastricht University]. Universitaire Pers Maastricht. https://doi.org/10.26481/dis.20130517rb

Document status and date:

Published: 01/01/2013

DOI:

10.26481/dis.20130517rb

Document Version:

Publisher's PDF, also known as Version of record

Please check the document version of this publication:

- A submitted manuscript is the version of the article upon submission and before peer-review. There can be important differences between the submitted version and the official published version of record.

People interested in the research are advised to contact the author for the final version of the publication, or visit the DOI to the publisher's website.

- The final author version and the galley proof are versions of the publication after peer review.

- The final published version features the final layout of the paper including the volume, issue and page numbers.

Link to publication

\footnotetext{
General rights rights.

- You may freely distribute the URL identifying the publication in the public portal. please follow below link for the End User Agreement:

www.umlib.nl/taverne-license

Take down policy

If you believe that this document breaches copyright please contact us at:

repository@maastrichtuniversity.nl

providing details and we will investigate your claim.
}

Copyright and moral rights for the publications made accessible in the public portal are retained by the authors and/or other copyright owners and it is a condition of accessing publications that users recognise and abide by the legal requirements associated with these

- Users may download and print one copy of any publication from the public portal for the purpose of private study or research.

- You may not further distribute the material or use it for any profit-making activity or commercial gain

If the publication is distributed under the terms of Article $25 \mathrm{fa}$ of the Dutch Copyright Act, indicated by the "Taverne" license above, 


\section{Impacts of Government Incentives to R\&D, Innovation and Productivity: \\ A Microeconometric Analysis of the Québec \\ Case}


(C) 2013 Rufin S. A. Baghana All rights reserved

Cover design by Datawyse Maastricht

Published by Universitaire Pers Maastricht

ISBN 9789461592262

Printed in the Netherlands by Datawyse Maastricht 


\section{Impacts of Government Incentives to R\&D, Innovation and Productivity: A Microeconometric Analysis of the Québec Case}

\section{DISSERTATION}

to obtain the degree of doctor at Maastricht University, on the authority of the Rector Magnificus Prof. Dr. L.L.G. Soete, in accordance with the decision of the Board of Deans, to be defended in public on Friday May $17^{\text {th }} 2013$, at $16: 00$ hours

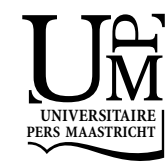




\section{Supervisor}

Prof. Dr. Pierre Mohnen

\section{Assesment Committee}

Prof. Dr. Bart Verspagen (chairman)

Prof. Dr. Isabel Busom (Universitat Autonoma de Barcelona, Spain)

Prof. Dr. Jacques Mairesse 
"Who lacks knowledge is being constantly at the mercy of change."

Rémi Belleau 


\section{Disclaimer:}

The views expressed in this book are the sole responsibility of the author and do not necessarily reflect those of the Ministère des Finances du Québec, the Government of Québec or the Government of Canada. Additionally, all errors and omissions remain the author's sole responsibility. While best efforts have been used in preparing this book, the author makes no representations or warranties of any kind and assumes no liabilities of any kind with respect to the accuracy or completeness of the contents. 


\section{Acknowledgements}

There is a popular proverb that says, "No matter how long the night is, the morning is sure to come." As I conclude this thesis, I would like to send these encouraging words to all those who are in the course of writing their theses, and especially to those who are still at the beginning of this process. It is true that, as the saying goes, this process can be very lonely. Working on this project will remain a memorable accomplishment for me, because each day represented a huge challenge, especially due to the need to reconcile family, work and thesis writing. The joys, sorrows and feelings of discouragement were confronted with the vicissitudes of this long desert crossing. There is a great bench of people behind this work, and I would like to thank them all from the bottom of my heart.

First and foremost, I would like to thank my advisor, Pierre Mohnen, who supported me over the years and gave me so much freedom to explore and discover new areas. Pierre, I would like to stress how much I enjoyed the way you have guided me through this research project. I was able to take advantage of your experience and availability, and these are capital elements when carrying out such a project. I don't think this is news to anyone who has already written a doctoral thesis. Since you were one of my favourite teachers during my master's studies at the University of Québec in Montreal, it was very easy for me to decide to come to Maastricht to complete my thesis. I would also like to highlight the contribution of Pierre Mohnen as co-author of a chapter in this thesis that was published as "Effectiveness of R\&D Tax Incentives in Small and Large Enterprises in Québec" in the journal Small business economics ((2009) 33:91-107).

I also thank the Institut de la Statistique du Québec (ISQ). Indeed, this thesis would not have been possible without the ISQ which provided me with access to the databases used in this project. This was a major challenge because most data was dictated by rules of strict confidentiality. To override this restriction, the ISQ had to make arrangements to allow access to the data while complying with federal and provincial rules and obtaining approval from both governments. Moreover, in addition to legal issues, there were all the difficulties related to data preparation, particularly with matching large and complex databases from different sources. Here again, I was able to benefit from the resources and expertise of the ISQ, and so, I want to thank any staff member at ISQ who, directly or indirectly, helped me in the various stages of this project. In this regard, I would especially like to thank Brigitte Poussart from the Science, Technology and Innovation team, whose contribution was crucial to the accomplishment of this project. She accompanied me through the long process to obtain authorizations from provincial and federal 
authorities to access the data. In addition to the logistical support, I also benefited from her knowledge and expertise, in particular, regarding the data sources used in this project and the creation of the final database. A thank you to Brigitte also for her meticulous proofreading and suggestions. Brigitte, I owe you one, and I am forever grateful for your contribution to this project.

I would also like to thank other members of the Science, Technology and Innovation team who contributed or helped me carry out this project. In particular, I thank Geneviève Renaud whose contribution as co-author of a chapter in this thesis is relevant. I thank Jean Berselli, whose expertise and knowledge helped me build part of the databases. Thank you also to Line Lainesse, Marguerite Simo, Marie-Eve St-Amand and Quynh-Van Tran. And of course, I cannot forget the head of this team, Camille Courchesne, and his successor, Pierre Cauchon, who supported this project as well.

I thank my superiors at the Ministry of Finance who encouraged me throughout this project, provided the logistical support and facilitated the reconciliation between work and the writing of this thesis. In particular, Pierre Dupont, Yves Lafrance, Denis Dufresne and Brigitte Duchesne. They find in these few words all my gratitude for their support.

I would like to thank my colleagues in Québec with whom I have had the pleasure of working over the years and whose contribution was very useful. Especially Michel Falardeau, who introduced me to the fiscal schemes for R\&D of the Government of Québec. I also benefited from useful information from Christian Perodeau, Louis Castonguay, Martin Picard, all from the Ministère des Finances du Québec, and Marie-Odile Koch from the Ministère du Développement économique, de I'Innovation et de l'Exportation. To Bernard Barrucco and Martial Audet, I would like to say thank you for your words of encouragement.

Finally, I could not finish without a word of thanks to my friends and relatives for their encouragement. I thank in particular Pie Landry lloud for his unfailing support, and Christian Tchimenga, Olivier Baghana, Davy Baghana and Aymar Baghana for their moral support.

June 2012 


\section{Contents}

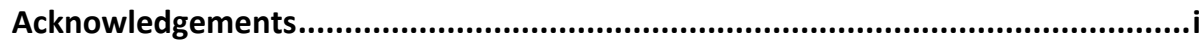

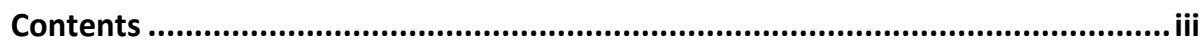

List of Figures ............................................................................................ vii

List of Tables ................................................................................................. ix

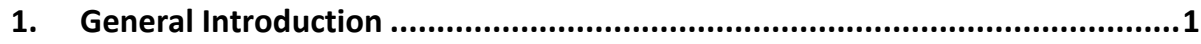

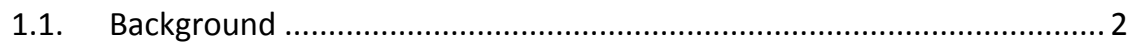

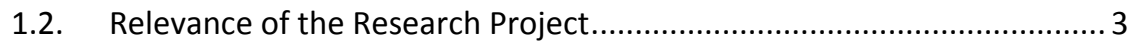

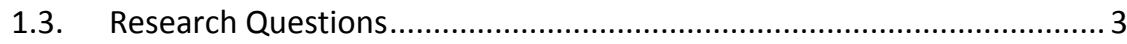

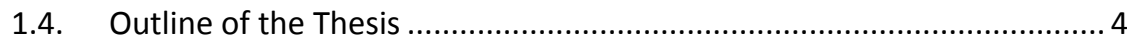

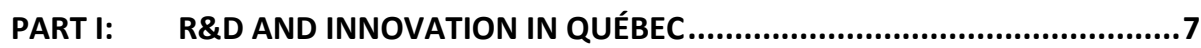

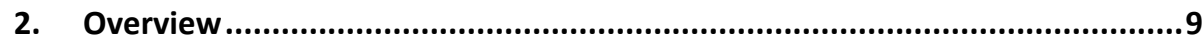

2.1. Defining R\&D and Innovation for the Purposes of Public Assistance Programs ........................................................................ 10

2.2. Rationale for Government Support................................................... 13

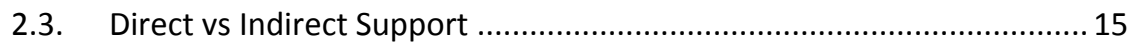

2.4. Institutional Environment ……………………….......................... 17

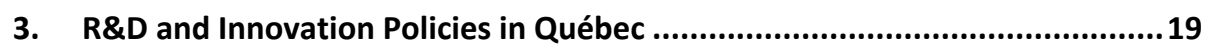

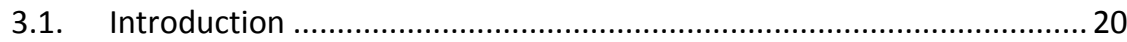

3.2. Historical Overview of R\&D and Innovation Policies in Québec ............ 21

3.2.1. From the 1960s to the beginning of the 1980s ..................... 22

3.2.2. Since the beginning of the 1980s ........................................... 23

3.3. Support Measures of the Government of Québec................................ 25

3.3.1. Indirect Support Measures ..................................................... 25

3.3.2. Direct Support Measures....................................................... 31

3.3.3. Total Costs for the Government of Québec ............................. 35

3.4. Support Measures of the Federal Government ......................................36

3.4.1. Indirect Support Measures .................................................... 36

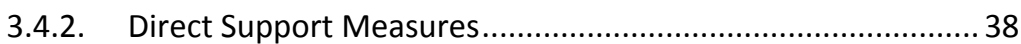

3.5. Net Cost of the Combined Federal-Provincial Indirect Support

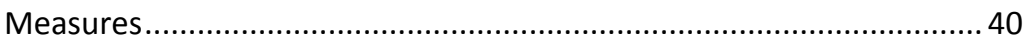

3.6. Interprovincial Comparison of the Main Tax Credits for R\&D................ 42

3.7. Statistics on R\&D and Innovation in Québec ...................................... 43

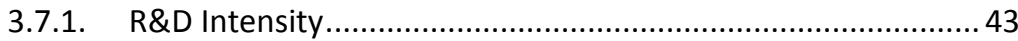




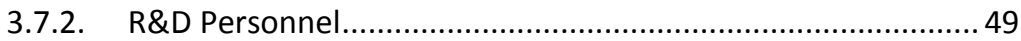

3.8. R\&D Intensity and the Québec's Productivity gap Issue .......................52

Chapter Appendices .................................................................................. 54

3.A. Science and Technology Policies in Québec: Key Highlights, 1960-

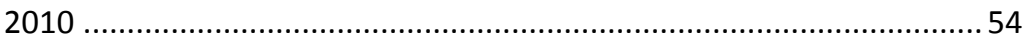

3.B. Summary of the Provincial and Federal Tax Credits Schemes ................ 57

3.C. Costs of R\&D and Innovation Measures...............................................65 65

3.D. Net Cost of R\&D Expenditures for Business Enterprises .......................68

3.E. Main Statistics on R\&D and Innovation......................................... 70

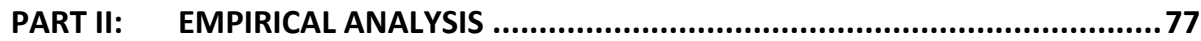

4. Data Sources and Construction of Datasets..............................................79

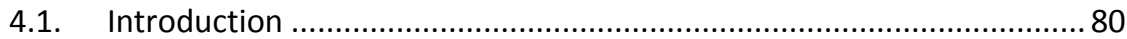

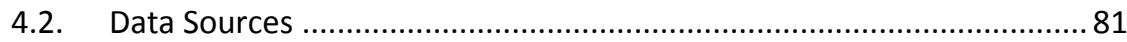

4.2.1. Survey of Research and Development in Canadian

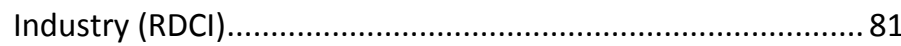

4.2.2. Annual Survey of Manufactures (ASM) .............................. 81

4.2.3. Canadian Survey of Innovation 2005................................... 82

4.2.4. Administrative Data from Revenu Québec (ADRQ) ................ 83

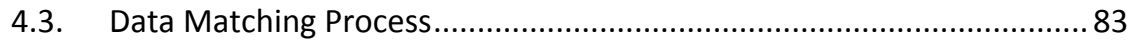

4.3.1. RDCl-ADRQ Matched Data.................................................... 83

4.3.2. RDCI-ADRQ-ASM Matched Data .......................................... 84

4.3.3. IS2005-ASM-RDCI-ADRQ Matched Data ................................ 86

5. Effectiveness of R\&D Tax Incentives in Manufacturing Sector in Québec...............................................................................................89

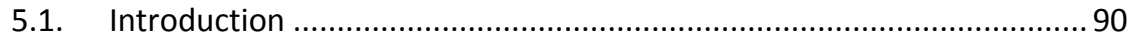

5.2. Data and Firms Characteristics..................................................... 92

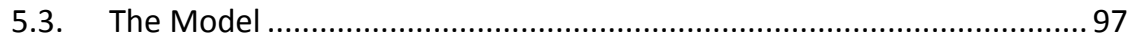

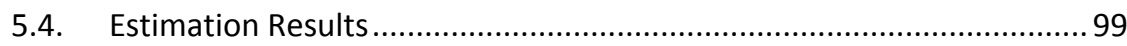

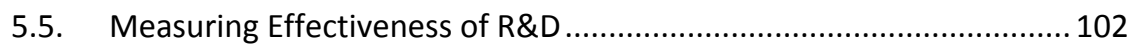

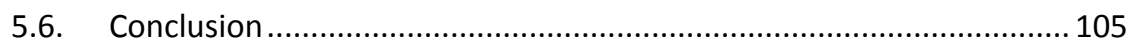

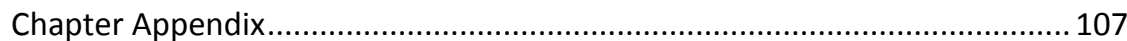

5.A. The User Cost of the R\&D in Québec ........................................... 107

6. Public R\&D Subsidies and Productivity: Evidence from Firm-Level Data

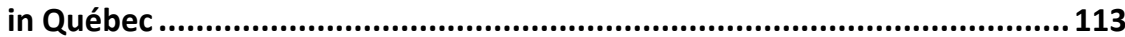

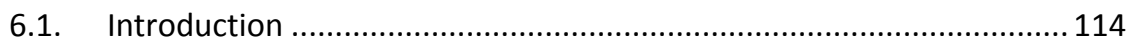

6.2. Public Direct Subsidies toward R\&D in Québec .................................. 116

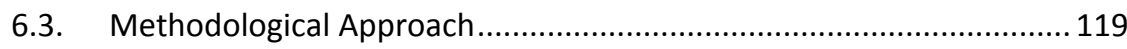

6.3.1. Step 1: The Matching Estimator Framework ....................... 120

6.3.2. Step2: The Production Function Framework....................... 124 


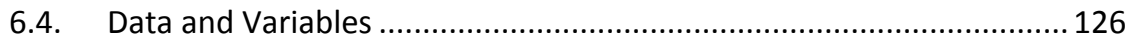

6.4.1. Data ............................................................................. 126

6.4.2. Variables for the Estimation of ATT .................................... 127

6.4.3. Variables Entering the Production Function......................... 128

6.5. Empirical Analysis and Results ..................................................... 129

6.5.1. Assessing the Input Additionality of R\&D Grants .................. 129

6.5.2. Assessing the Impact of Privately and Publicly Funded R\&D on Productivity Growth .......................................... 137

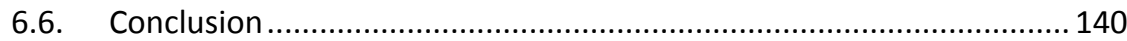

Chapter Appendices ......................................................................... 142

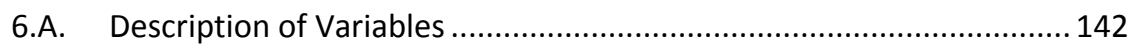

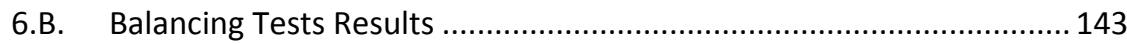

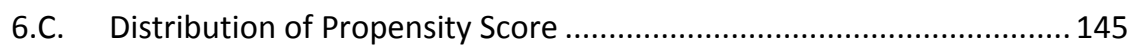

6.D. Classification of Manufacturing Industries......................................... 147

6.E. Derivation of the Labour Productivity Equation ............................... 149

7. Is Private R\&D Spending Sensitive to Increments in Fiscal incentives? Evidence from the Superdeductions Program in Québec .......................... 151

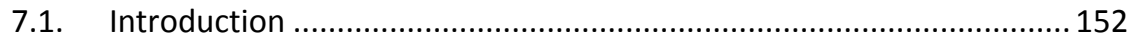

7.2. General Portrait of the Program of Superdeductions ......................... 153

7.2.1. Eligibility for Assistance ........................................................ 153

7.2.2. Nature of Assistance......................................................... 153

7.2.3. Cost of Superdeductions and Characteristics of Firms receiving the Superdeductions ............................................. 155

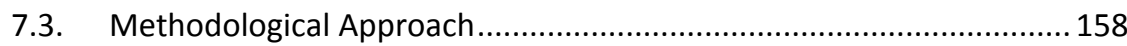

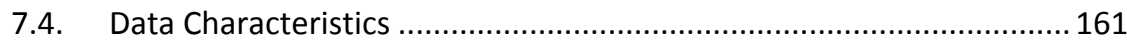

7.5. Assessing the Impact of the Program of Superdeductions ................. 162

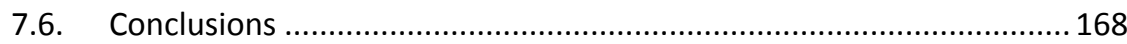

8. Impact of Government Funding on R\&D, Innovation, and Productivity: Results from the Innovation Survey Microdata in Québec........................ 169

8.1. Introduction ............................................................................. 170

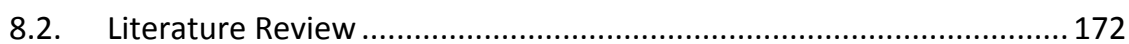

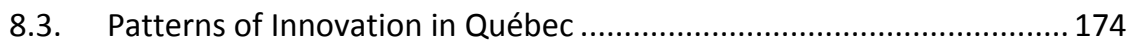

8.3.1. Characteristics of Innovative Firms ..................................... 174

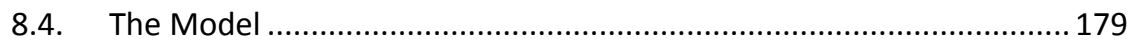

8.4.1. The OECD Core Model ........................................................ 179

8.4.2. Extending the OECD Model: The Québec Model................... 181

8.5. Data and Variables ............................................................................. 183

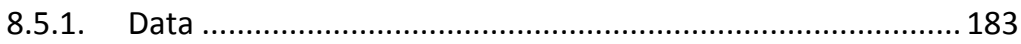

8.5.2. Correspondance CIS 4 - IS2005 ....................................... 183

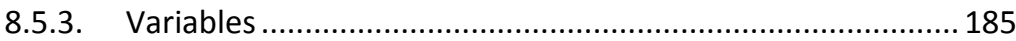




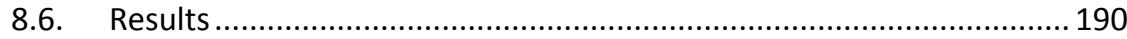

8.6.1. Results of the Québec Extended Model ............................... 190

8.6.2. Comparing Québec to International Econometric Findings (OECD Model) .................................................. 201

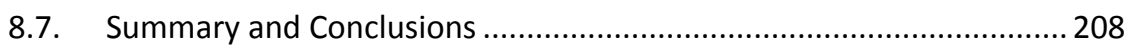

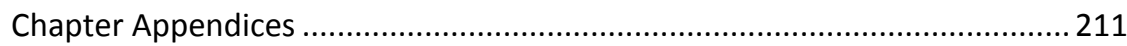

8.A. Descriptive Statistics of Groups ........................................................ 211

8.B. Econometric Findings from the Extended Model for Québec (SubSamples) .................................................................................... 213

8.C. Main Economic Regions of Québec................................................... 214

8.D. Survey of Innovation 2005 - Questionnaire and Corresponding variables ............................................................................................... 215

8.E. CIS 4 Questionnaire and Corresponding Variables .......................... 233

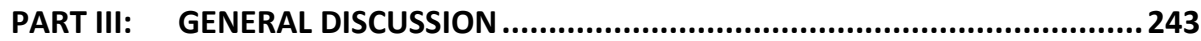

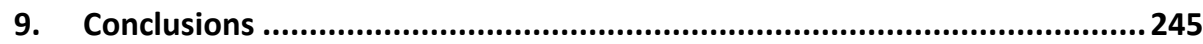

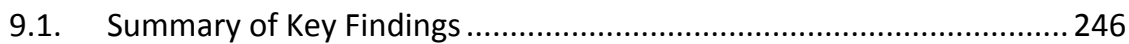

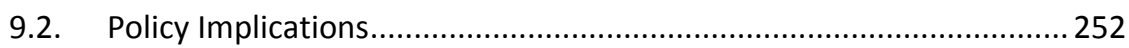

9.3. Limitations and Suggestions for Future Research ............................. 254

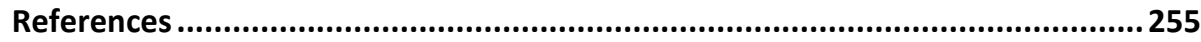

List of Abbreviations and Acronyms ..................................................... 265

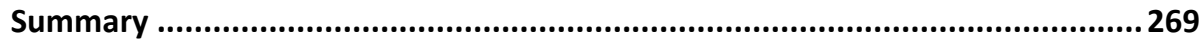

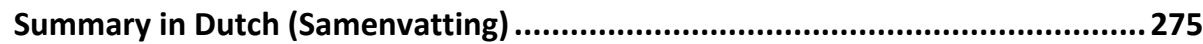

Summary in French (Resumé) ........................................................... 281 


\section{List of Figures}

Figure 2.2-1: The private and social return to $R \& D$

14

Figure 2.3-1: Direct and indirect government funding of business R\&D and tax incentives for R\&D, 2007. 16

Figure 3.3-1: Evolution of the estimated cost of fiscal incentives for R\&D and innovation, 1997-2007

Figure 3.3-2: Distribution of the costs of direct programs for R\&D, by sectors, 2003-2004

Figure 3.3-3: Distribution of the costs of direct programs for innovation, by sectors, 2003-2004

Figure 3.3-4: Distribution of the costs of $R \& D$ and innovation programs, total direct and indirect measures, 2003-2004 35

Figure 3.3-5: Comparative evolution of the total R\&D expenditures of Canadian provincial governments, 1994-1995 to 2002-2003

Figure 3.4-1: Federal expenditure on direct programs for $R \& D$, by sectors, 2003-2004

Figure 3.4-2: Evolution of Federal expenditure on direct programs for $R \& D$, by sectors, 2003-2004 to 2008-2009 40

Figure 3.5-1: Rate of fiscal subsidy for a dollar of R\&D, Québec, Ontario,

Canada and selected countries, large firms, 2006-2007

Figure 3.5-2: Rate of fiscal subsidy for a dollar of R\&D, Québec, Ontario, Canada and selected countries, SMEs, 2006-2007

Figure 3.7-1: Evolution of R\&D intensity, Québec and Canadian provinces, 1981-2008.

Figure 3.7-2: R\&D intensity, Québec and Canadian provinces, 2008 45

Figure 3.7-3: Evolution of real GERD, by sector, Québec, 1981-2008 46

Figure 3.7-4: Comparison of R\&D intensity, by performer, Canada, Québec and Ontario, 2008.

Figure 3.7-5: Evolution of R\&D intensity, business sector, Québec and Canadian provinces, 1981-2008

Figure 3.7-6: Evolution of R\&D intensity, Québec, Canada and selected OECD countries, 1997-2008. 
Figure 3.7-7: Evolution of R\&D intensity, business sector, Québec, Canada and selected OECD countries, 1997-2008

Figure 3.7-8: Proportion of R\&D personnel, by Sector, Québec, Canada and selected provinces, 2005 . 50

Figure 3.7-9: Ratio of researchers to R\&D personnel, all sectors, Canada and provinces, 2005

Figure 3.7-10: Ratio of researchers to R\&D personnel, business sector, Canada and provinces, 2005

Figure 5.5-1: Evolution of the bang for the buck when increasing by $10 \%$ the level-based provincial rate of R\&D tax credit..... 105

Figure 6.C-1: Distribution of propensity score before and after matching, all firms, 1998-2004 145

Figure 8.4-1: The OECD core model and the extended model for Québec 183 


\section{List of Tables}

Table 2.4-1: Main federal and provincial bodies funding R\&D and innovation of corporations

Table 3.3-1: $\quad$ Rates applicable to superdeductions ........................................... 28

Table 3.4-1: $\quad$ Estimate of tax expenditure, total Canada, 2005-2010..................... 38

Table 3.B-1: $\quad$ Summary of the Québec's SR\&ED tax credit rates, 2010 rates .........57

Table 3.B-2: $\quad$ Summary of the Federal SR\&ED tax credit rates, 2010 rates ........... 58

Table 3.B-3: $\quad$ Summary of provincial indirect measures for Innovation ................ 59

Table 3.B-4: Interprovincial comparison of the main tax credits for R\&D............61 61

Table 3.C-5: Estimated cost of fiscal incentives for R\&D and innovation, Québec, 1997-2007

Table 3.C-6: Cost of direct programs for R\&D and innovation, 2000-2001 to 2003-2004

Table 3.C-7: $\quad$ Cost of direct programs for R\&D, federal Government, 20032004 to $2008-2009$

Table 3.C-8: Total expenditures of provincial governments on R\&D, 1994 1995 to $2002-2003$

Table 3.D-9: Net cost of $\$ 100,000$ R\&D expenditures incurred in Québec by an SME in the manufacturing sector, 2003 tax rates

Table 3.D-10: Net cost of $\$ 100,000$ R\&D expenditures incurred in Québec by a large corporation or a foreign-controlled corporation in the manufacturing sector, 2003 tax rates

Table 3.E-11: Gross domestic expenditure on R\&D (GERD), percentage of GDP, Canada and Canadian provinces, 1981-2008

Table 3.E-12: Business expenditure on R\&D (BERD), percentage of GDP, Canada and Canadian provinces, 1981-2008

Table 3.E-13: GERD, percentage of GDP, Québec, Canada and OECD countries, 1997-2008.

Table 3.E-14: BERD, percentage of GDP, Québec, Canada and OECD countries, 1997-2008.

Table 3.E-15: GERD, by performer, Québec, 1981-2008, 2002 constant prices 
Table 3.E-16: R\&D Personnel, Québec, Ontario and Canada, 2002 to 2008 75

Table 3.E-17: R\&D Personnel, by Sector, Canada and selected provinces, 2005 75

Table 4.3-1: Summary of matching success in first step and R\&D expenditures in samples

Table 4.3-2: $\quad$ Summary of matching success in the second step and R\&D expenditures in samples

Table 5.2-1: Use of Québec's R\&D tax credits by size class in the manufacturing sector (\% of firms), 1997-2003. 95

Table 5.2-2: Use of Québec's R\&D tax credits by industry group in the manufacturing sector (\% of firms), 1997-2003. 96

Table 5.4-1: Variable constructions and descriptive statistics 99

Table 5.4-2: Estimation results of R\&D equation 101

Table 5.5-1: $\quad$ Trajectory of R\&D flows and stocks under two scenarios 102

Table 5.A-1: Estimated annual average user ost of R\&D, all firms, 19972003 109

Table 5.A-2: Chapter symbols and parameter values. 110

Table 6.2-1: $\quad$ Comparative evolution of public direct incentives and tax credits aimed at R\&D in the manufacturing sector in Québec, 1997-2004

Table 6.2-2: Use of public grants by size class in the manufacturing sector, 1997-2004

Table 6.2-3: Use of public grants by technology level, 1997-2004 118

Table 6.5-1: Description of the samples used in the matching 130

Table 6.5-2: Probit estimates for the propensity score estimation, all firms, 1998-2004

Table 6.5-3: Probit estimates for the propensity score estimation, Lowmedium technology firms, 1998-2004

Table 6.5-4: Probit estimates for the propensity score estimation, High technology firms, 1998-2004.

Table 6.5-5: Estimated effect of treatment on R\&D intensity based on conditional difference-in-differences and local regression matching

Table 6.5-6: Estimation results of OLS-HC and GMM models for the productivity growth, all firms 
Table 6.A-1: Variables construction and descriptive statistics

142

Table 6.B-2: Balancing tests before propensity score matching for the average effect of treatment on R\&D intensity, all firms, 19982004

Table 6.B-3: Balancing tests after propensity score matching for the average effect of treatment on R\&D intensity, all firms, 19982004

Table 6.D-4: OECD Classification of manufacturing industries based on technology using ISIC rev. 3 activity breakdown

Table 6.D-5: Classification of manufacturing industries based on the type of activity using NAICS 3 digit code breakdown

Table 7.2-1: $\quad$ Superdeductions rates vs SR\&ED tax credit rates, 1999 rates 154

Table 7.2-2: Comparison between the superdeduction and SR\&ED tax credit: net cost for a corporation of an expenditure of $R \& D$ salaries of CAN \$100 incurred in Québec, 1999

Table 7.2-3: Cost of superdeductions and of other fiscal incentives to R\&D granted by the Government of Québec and number of recipients enterprises, $1999-2002$.

Table 7.2-4: Distribution of the value of superdeduction by size class, 2000 .... 157

Table 7.2-5: Distribution of the value of superdeduction by sectors, $2000 \ldots . . .157$

Table 7.4-1: Number of superdeductions and of other fiscal incentive recipients to $R \& D$ in the study sample, 1998-2002.

Table 7.5-1: Probit estimates for the propensity score estimation. 163

Table 7.5-2: Estimated average treatment effects (ATT) on R\&D intensity change 165

Table 7.5-3: Balancing tests before propensity score matching, all firms..... 166

Table 7.5-4: Balancing tests after propensity score matching, all firms. 167

Table 8.3-1: Distribution of innovating firms in Québec's manufacturing sector, 2002-2004.

Table 8.3-2: Product innovations distribution following STAN aggregation, 2002-2004.

Table 8.3-3: Innovation rate by technology level, 2002-2004 .......................... 177

Table 8.3-4: Innovation rate by size class, 2002-2004 ...................................... 177

Table 8.3-5: Innovation rate by geographic localization, 2002-2004 …............. 178

Table 8.3-6: Firms usage of government sponsored programs, 2002-2004....... 178 
Table 8.5-1: Correspondance between CIS 4 and IS2005.

Table 8.5-2: Causality of the OECD core model and the extended model for Québec

Table 8.5-3: Variables construction and sample descriptive statistics, all firms.

Table 8.6-1: Description of the samples for the Québec extended model 191

Table 8.6-2: Results for the Québec extended model, first stage, full sample

Table 8.6-3: $\quad$ Results for the Québec extended model, second and third stages 200

Table 8.6-4: Which firms are more likely to be innovative? ..............................2 204

Table 8.6-5: Which firms spend more on innovation? ....................................... 206

Table 8.6-6: What is the impact of product innovation on labour productivity?................................................................................. 207

Table 8.A-1: Group means comparison between innovators and noninnovators, total sample............................................................ 211

Table 8.B-2: Findings from SME's and technology industries samples.............. 213

Table 8.C-3: Main economic regions of Québec............................................ 214 
CHAPTER 1

General Introduction 


\subsection{Background}

Economic theory teaches that productivity growth is the fundamental determinant of the growth of a country's standard of living. Increasing labour productivity depends on investment and saving, physical capital, human capital and new technology. For a long time, economists and policy makers have been interested in this last factor, in particular in how to increase the stock of knowledge from which new technologies emerge. One way to do this is to increase investment in R\&D and other innovation activities. However, for obvious economic reasons, the private sector does not necessarily realize the required level of investment in R\&D or does not cover all sectors or all innovations needed. Thus, the government's use of incentives to encourage private investment in $R \& D$, either by direct methods such as grants or indirect methods such as tax credits, has become essential to dealing with this issue. In recent decades, governments, mainly in industrialized countries, have implemented significant direct or indirect methods of intervention, or a combination of both depending on the country, within a context of more or less structured national policies designed to encourage the private sector, mainly business enterprises, to increase their R\&D investment. Over the years, companies have been subsidized to amounts totaling billions of dollars annually in several OECD countries.

However, given the fact that the additionality of government support is not directly observable, the government spending in R\&D and innovation raises a debate that remains open regarding its effectiveness. Criticisms were voiced about the effects of government spending on private R\&D investment and firms' productivity. It is frequently questioned whether an increase in government spending crowds out private investment. In terms of policy evaluation, the debate continues and critics raise methodological weaknesses due to the fact that the models used in the assessment of the effectiveness of government spending do not reflect the heterogeneity of the public support received by companies.

Through exploration of recent developments in econometrics and using data from Québec firms, this study attempts to assess the impacts of government incentives on private investment of $R \& D$, especially that of business enterprises, and on their innovative performance and productivity. Although Québec is part of the Canadian federation, a study based on Québec data is interesting in several regards. First, Québec owns and administers its own policies and programs to encourage R\&D and innovation and its economy is comparable to, if not more important than, that of many industrialized countries. Therefore, the issues mentioned above arise to the same degree. Second, for a long time Canada has been recognized as one of the countries in the world offering the most 
advantageous R\&D package to private business and this is, in part, attributable to the province of Québec, which offers, as will be shown in upcoming chapters, the most advantageous R\&D incentives in Canada. Third, as just mentioned, despite the fact that Québec offers incentives for R\&D among the largest in the world, its case is puzzling, as it continues to lag far behind its neighbors with regards to productivity. Fourth, in recent years, particularly in the context of the global recession, voices were raised in Canada against the high growth of expenditures for incentives to $R \& D$ payments to businesses, which are considered as already benefiting largely from the tax advantages available to corporations. Fifth and finally, there is a gap in empirical studies on the issues raised above with regards to Québec. To date, the few econometric studies concerning the evaluation of public support for innovation in Québec have often been conducted within Canada-wide analyses. Moreover, these studies are mostly limited to assessing the impact of induced tax credits on private R\&D funding.

\subsection{Relevance of the Research Project}

The originality of this study comes at least from two aspects: first, it exploits the actual R\&D tax credits and subsidies received by every firm, instead of just relying on the statutory tax rates and eligibility conditions and then attributing incentives to every eligible firm. Second, to date, no study of this magnitude has been conducted on data from Québec. It is certainly true that only a few years ago, such a project would not have been possible given the lack of information that prevailed. However, in recent years, significant efforts undertaken by Statistics Canada, the Institut de la statistique du Québec and Revenu Québec (formerly the Ministère du Revenu du Québec) have helped fill this gap. Also, recent developments in the literature of econometric evaluation and applied statistics were decisive factors for conducting this project.

\subsection{Research Questions}

As mentioned above, this study aims to empirically assess the effectiveness of direct and indirect measures of government support to R\&D and innovation on both the private investment of firms in $R \& D$ and on their economic performance, in particular in terms of innovation output and labour productivity. This is a broad subject from which several research questions may be explored. However, expectations in this perspective are often confronted to the issue of data availability. In our case, a thorough examination of the data allowed to cover a wide 
range of relevant questions to help shed light on the subject of research. Following are the eight research questions developed:

1) Does fiscal policy subsidizing salaries and wages of researchers translate into increased ratios of researchers in total employment? (Chapter 3)

2) Are R\&D tax incentives (indirect incentives) effective in Québec? In particular, how much private R\&D increases per dollar of $R \& D$ tax receipts foregone? (Chapter 5)

3) Are level-based R\&D tax incentives more efficient than incremental tax incentives? (Chapter 5)

4) Is private R\&D spending sensitive to increments in fiscal incentives? (Chapter 7)

5) Do public subsidies (direct incentives) increase private $R \& D$ investment? How do these incentives impact firms' productivity? (Chapter 6)

6) Are the combinations of public intervention methods currently used in Québec to encourage R\&D optimal? (Chapter 6)

7) What is the impact of overall public incentives (direct and indirect) on firms' $R \& D$, innovation, and productivity in Québec? How do firms in Québec compare to firms in other jurisdictions? (Chapter 8)

8) Can we attribute the causes of the lag experienced by Québec in its labour productivity to the underperformance of firms in innovation? (Chapter 3 and Chapter 8)

\subsection{Outline of the Thesis}

In Québec, businesses that undertake R\&D and innovative activities benefit from strong federal and provincial support. Various public incentive programs for R\&D and innovation have been available to these firms for decades. In this project our goal is to assess whether firms respond adequately to these incentives and whether the incentives in place are optimal. To achieve this, we use longitudinal firm level data, not only sourced from survey data, but also from administrative data. As is often the case, a firm located in Québec can receive during a given year several types of government support from both the governments of Canada and Québec. This research project does not attempt to distinguish, in the first place, the impact of government support that each government provides firms. This project rather focuses on how firms respond to different types of government incentives. Thus, an effort is made to isolate the effect of direct government incentives (subsidies, 
grants) to those of indirect government incentives (tax credits, fiscal deductions). It is this element that determined the structure of this thesis as presented below.

In addition to this introductory chapter, this research project contains eight chapters divided into three parts: the first part, consisting of Chapters 2 and 3, briefly defines the main concepts used and presents a review of policies for R\&D and innovation in Québec and the main R\&D and innovation indicators. The second part consists of Chapters 4, 5, 6, 7 and 8 and is devoted to the empirical analysis of the research questions presented above. Lastly, the third part, consisting solely of Chapter 9, contains the conclusions and policy recommendations. Here is a more detailed description of Chapters 2-9:

Chapter 2, Overview, defines some key concepts such as "research and experimental development" according to the legislation applicable in Québec. It also addresses the justification of government support to R\&D and innovation, and finally, presents the provincial and federal administrative environment in which companies conduct business.

Chapter 3, R\&D and Innovation Policies in Québec, first presents a historical overview of R\&D and innovation policies in Québec. It then presents an in-depth review of direct and indirect incentives, including their costs, offered to R\&D performers and innovators located in Québec by the governments of Québec and Canada. The emphasis, however, is rather on the policies of the Government of Québec than on those of the Government of Canada since the former are more specific to Québec firms. This chapter includes an interprovincial comparison of the $R \& D$ incentives offered to firms as well as a presentation of the statistics on $R \& D$ and innovation in Québec, in particular those linked to private and public investments in R\&D and innovation. A comparison at the global scale is also presented in order to situate Québec in the world. Finally, we discuss the R\&D intensity and the issue of Québec's productivity gap in light of the statistics.

Chapter 4, Data Sources and Construction of Datasets, describes the sources of databases and the construction of datasets used in the empirical analysis. The approach used and the hurdles encountered in the process of linking the files from Statistics Canada surveys and administrative data from Revenu Québec are addressed. The results of the matching process data are presented as well.

Chapter 5, Effectiveness of R\&D Tax Incentives in Manufacturing Sector in Québec, evaluates the effectiveness of R\&D tax incentives in Québec, using manufacturing firm data from 1997 to 2003 originating from R\&D surveys, annual surveys of manufactures and administrative data of the actual amounts of government support. Relying on the estimation of a structural factor demand model for R\&D with R\&D tax parameters included in the user cost of R\&D, an evaluation of the so-called "bang for the buck" cost-benefit analysis that permits to estimate how much private R\&D increases per dollar of R\&D tax receipts foregone is performed. This is done by running simulation analyses of the effects of short-run and long-run 
tax changes on small and large firms in order to show differences in the effectiveness of R\&D tax incentives between both groups. In addition, simulation analyses of $R \& D$ tax experiments to calculate the ratio of additional R\&D per dollar of tax expenditure for different modules of the R\&D tax credit are performed in order to assess whether a deadweight loss is associated with these modules.

Chapter 6, Public R\&D Subsidies and Productivity: Evidence from Firm-Level Data in Québec, empirically analyzes the impacts of public R\&D grants on private $R \& D$ investments and on the productivity growth of the manufacturing firms in a context where fiscal incentives are present. The methodological approach is performed in two stages: first, a conditional semiparametric difference-indifferences estimator on longitudinal data from Québec is used to assess the comparative performance of firms that use public grants in conjunction with tax credits and firms that use only tax credits in terms of R\&D input additionality. Second, a production function is used to assess the effectiveness of public R\&D grants in terms of the productivity growth of firms.

Chapter 7, Is Private R\&D Spending Sensitive to Increments in Fiscal Incentives? Evidence from the Superdeductions Program in Québec. In this chapter we seek to determine the impact of superdeductions on the R\&D efforts of companies who chose this option. Specifically, we seek to determine whether companies receiving superdeductions have passed the surplus tax gain of this new aid provided in addition to the tax credits on to their R\&D spending.

Chapter 8, Impact of Government Funding on R\&D, Innovation, and Productivity: Results from the Innovation Survey Microdata in Québec, evaluates the impacts of government funding on innovation investments, on firms' innovative sales (innovation output) and on labour productivity using data from manufacturing firms in Québec from 2002 to 2004. An econometric selection model in which government incentives may increase the profit from innovating is used. This model is developed from a multicountry model constructed by an OECD team from the Crépon, Duguet and Mairesse (1998) framework. In a subsequent step, the performance of manufacturing firms from Québec is compared internationally in terms of innovation output and labour productivity using an adapted version of the OECD model to Québec data. From the estimation results, the issue of the lag experienced by Québec in its labour productivity is also addressed.

Chapter 9, Conclusions, first summarizes the impact assessment of government incentives and the main issues of R\&D and innovation framework and policies in Québec. It then presents findings for the specific research questions explored. Lastly, it presents policy implications and draws avenues for future research.

Before closing this chapter, it is important to note that, since some chapters of this thesis have been submitted or published as individual papers, there might be some overlap between the chapters. 
PART I

R\&D AND INNOVATION

IN QUÉBEC 

CHAPTER 2

Overview 


\subsection{Defining R\&D and Innovation for the Purposes of Public Assistance Programs}

Contrary to some members of the Organisation for Economic Co-operation and Development $(\mathrm{OECD})^{1}$, fiscal legislations in Canada (federal or provincial) aimed at innovation activities do not provide a definition of the term "technological innovation". The Canadian Income Tax Act rather defines the term "scientific research and experimental development". Hence, to ensure uniformity in the determination of firms' eligible R\&D related expenditures, also refer to as Qualified Research Expenditure (QR\&E), a single definition of scientific research and experimental development (SR\&ED) in accordance with the guidelines of the OECD Frascati Manual (OECD (2002)), first published in 1963, has been enacted and used by the fiscal authorities of both the Government of Québec and the Canadian federal government.

Definition of scientific research and experimental development (based on the Canadian Income Tax Act (R.S.C., 1985, c. 1 (5th Supp.)))

"[S]cientific research and experimental development" means systematic investigation or search that is carried out in a field of science or technology by means of experiment or analysis and that is

(a) basic research, namely, work undertaken for the advancement of scientific knowledge without a specific practical application in view,

(b) applied research, namely, work undertaken for the advancement of scientific knowledge with a specific practical application in view, or

(c) experimental development, namely, work undertaken for the purpose of achieving technological advancement for the purpose of creating new, or improving existing, materials, devices, products or processes, including incremental improvements thereto,

and, in applying this definition in respect of a taxpayer, includes

\footnotetext{
${ }^{1}$ In recent years, authorities in some OECD countries, such as Spain, have begun to incorporate in their tax legislation the Oslo Manual definition of innovation in order to target the technological innovation as a single issue within a combined tax incentive policy, rather than targeting different innovation activities with separate policies.
} 
(d) work undertaken by or on behalf of the taxpayer with respect to engineering, design, operations research, mathematical analysis, computer programming, data collection, testing or psychological research, where the work is commensurate with the needs, and directly in support, of work described in paragraph $(a),(b)$, or $(c)$ that is undertaken in Canada by or on behalf of the taxpayer,

but does not include work with respect to

(e) market research or sales promotion,

(f) quality control or routine testing of materials, devices, products or processes,

(g) research in the social sciences or the humanities,

(h) prospecting, exploring or drilling for, or producing, minerals, petroleum or natural gas,

(i) the commercial production of a new or improved material, device or product or the commercial use of a new or improved process,

(j) style changes, or

(k) routine data collection.

Using a single definition ensure uniformity in the administration of public programs from both levels of government. To that end, as firms claiming tax credits in Québec are also tax claimants at the federal level government, Revenu Québec arranged with the federal taxation agency that is, Canada Revenue Agency, to apply the same definition for eligible R\&D activities. Furthermore, to ease its application at both levels, QR\&E are solely determined by Canada Revenue Agency.

Concerning innovation, even if the tax code does not provide a definition per se, provincial and federal authorities responsible for the administration of government programs use a definition based on the OSLO manual. This research project focuses mainly on the two broad forms of innovation, namely technological product innovation and technological process innovation, which were defined in the $2^{\text {nd }}$ edition of the Oslo Manual (OECD (1997)) as follows:

"A technological product innovation is the implementation/commercialisation of a product with improved performance characteristics such as to deliver objectively new or improved services to the consumer. A technological process innovation is the implementation/adoption of new or significantly improved 
production or delivery methods. It may involve changes in equipment, human resources, working methods or a combination of these."

The new edition of the Oslo manual broadens this definition and adds two additional forms of innovation, marketing and organizational innovation, such as follows:

\section{Definition of Innovation (Based on “Oslo Manual”, $3^{\text {rd }}$ edition, OECD (2005))}

An innovation is the implementation of a new or significantly improved product (good or service), or process, a new marketing method, or a new organizational method in business practices, workplace organization or external relations.

The minimum requirement for an innovation is that the product, process, marketing method or organizational method must be new (or significantly improved) to the firm.

Innovation activities are all scientific, technological, organizational, financial and commercial steps which actually, or are intended to, lead to the implementation of innovations. Innovation activities also include R\&D that is not directly related to the development of a specific innovation.

An innovative firm is one that has implemented an innovation during the period under review.

Main types of innovation:

1) A product innovation is the introduction of a good or service that is new or significantly improved with respect to its characteristics or intended uses. This includes significant improvements in technical specifications, components and materials, incorporated software, user friendliness or other functional characteristics. Product innovations can utilize new knowledge or technologies, or can be based on new uses or combinations of existing knowledge or technologies.

2) A process innovation is the implementation of a new or significantly improved production or delivery method. This includes significant changes in techniques, equipment and/or software. Process innovations can be intended to decrease unit costs of production or delivery, to increase quality, or to produce or deliver new or significantly improved products.

3) A marketing innovation is the implementation of a new marketing method involving significant changes in product design or packaging, product placement, product promotion or pricing. Marketing innovations are aimed at better 
addressing customer needs, opening up new markets, or newly positioning a firm's product on the market, with the objective of increasing the firm's sales.

4) An organizational innovation is the implementation of a new organizational method in the firm's business practices, workplace organization or external relations. Organizational innovations can be intended to increase a firm's performance by reducing administrative costs or transaction costs, improving workplace satisfaction (and thus labor productivity), gaining access to non-tradable assets (such as non-codified external knowledge) or reducing costs of supplies.

\subsection{Rationale for Government Support}

For a long time, economists explained the government involvement in technological advance and innovation activities by the Arrow-Nelson rationale, which is the key argument of the neo-classical approach of market failure (Nelson (1959), Arrow (1962)). The neo-classical approach of market failure focuses in the inefficiency of the market in generating to the whole economy a sufficient supply of knowledge in order to achieve optimality. This unattractive feature of the market is due principally to the fact that, in general, a social rate of return from R\&D is higher than the private rate of return leading to an imperfect appropriation of returns by innovative firms. In the other hand, R\&D activities have also the inherent feature of uncertainty. These elements lead to an underinvestment from private firms in R\&D leading to a supply offer of knowledge in the economy that is less than what would be socially desirable (OECD (1998). The Arrow-Nelson argument identifies three basic factors that prevent the realisation of a social optimum through private profit optimisation:

- Outcomes of knowledge generating processes are uncertain.

- Knowledge is a quasi-public good, implying inappropriability.

- There are substantial indivisibilities in knowledge generation.

The Arrow-Nelson rationale led to policy recommendation favoring the implementation of public framework to correct market failures by providing funding to innovation activities. However, this raised the question of knowing what would be the optimal subsidy for innovation activities and how will it be measured? An answer may be given intuitively from a graphical interpretation of the the private and social return to R\&D by Hall (1993). In Figure 2.2-1, the vertical and horizontal axes represent the marginal return to the last $R \& D$ dollar and the level of $R \& D$ projects respectively. The private return is represented by a downward sloping curve to take into account the diminishing marginal returns that face firms as the 
best projects are taken. The cost is represented by an upward sloping curve to capture the expected rising cost of each additional dollar. Firms will choose only all projects that fall to the left of point $\mathrm{C}$ as the anticipated return of such projects is higher than their costs. However, from point $C$ which is the equilibrium point of firms facing such cost curve, returns to society are higher than those received by firms. From that point it is clear that reliance on market processes alone will result in an underinvestment in R\&D from a social point of view. In order to get firms undertake the optimal level of R\&D, it is necessary to implement a subsidy to move firms from point Rc to point Rs in a way that the private rate of return equals the social rate of return at the socially optimal level of R\&D spending. The cost between point $P$ and $S$ is the optimal subsidy. Thus, in other words, government intervention raises the firm's private returns towards the higher rate of return of the economy as a whole, thus increasing R\&D levels over time to the optimum level for the society which is at point $S$.

Figure 2.2-1: The private and social return to R\&D

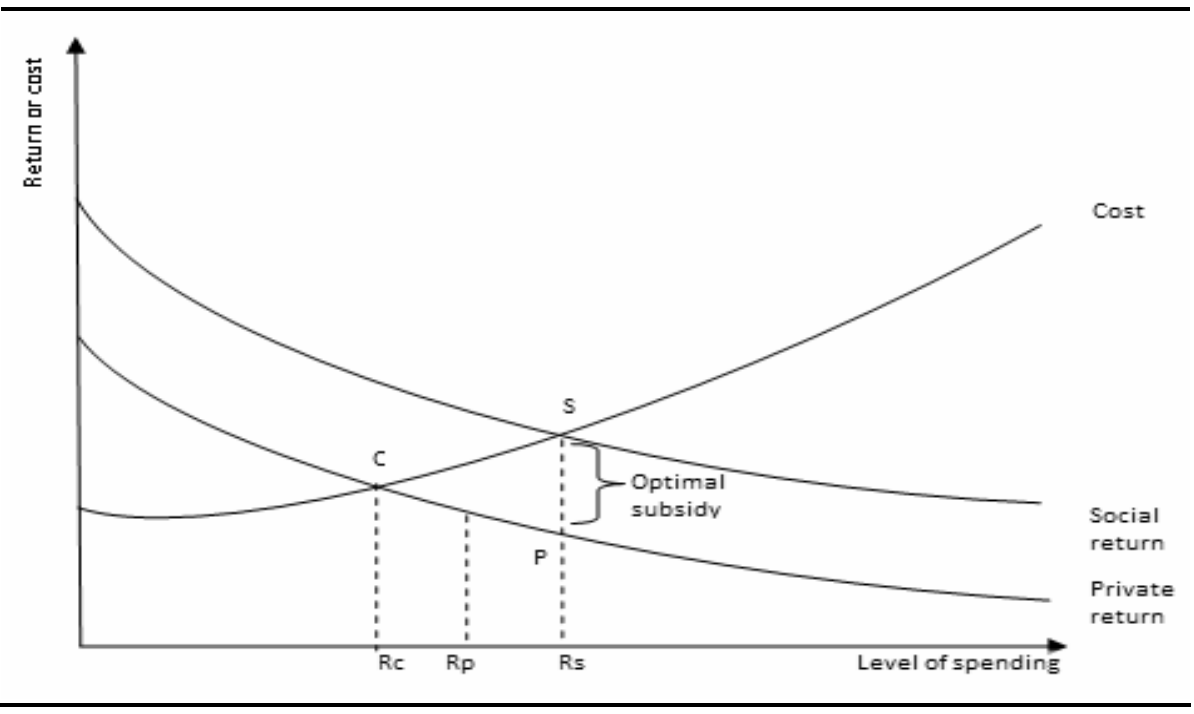

As outlined by Hall, the problem with the optimal subsidy is that this is a theoretic point that is in fact a counterfactual so it is not known. Hence, this point may vary across countries. Trying to answer to the first question, several countries now set targets with the goal of leveraging investment in R\&D. This is for example the case for Member States of the European Union that have set national investment targets in 2005 to be met in 2010 following the strategic goal fixed by the European Council in Lisbon in 2000 - the "Lisbon strategy" - and Barcelona in 
2002 aiming to raise overall R\&D investment to $3 \%$ of GDP by $2010^{2}$, of which two thirds should be financed by the business sector and one third by the public funds (OECD (2011)). In this setting, the efficient use of public support measures is viewed as a precondition in order to contribute to this objective (Dinges, Berger, Frietsch and Kaloudis (2007)).

Another problem is that once granted, the firm might just take the subsidy, but not increase its spending in R\&D. It could also affect some of the money received to other activities. As can be seen from the graph, in such a situation the effect of the subsidy policy is less, because the firm moves only to the point Rp, rather than to point Rs. This is a classic case of crowding out, as it will be explained in more details in the next section, which often leads to the following question: How much bang for the buck gets the society from the public Subsidies? Chapters 5 to 8 provide new evidence to this question.

The Department of finance Canada (Department of Finance (2010b)) justifies the SR\&ED tax credit in the following terms: "The federal income tax incentives for SR\&ED provide broadly based support for all types of SR\&ED performed in every industrial sector in Canada. The rationale for this tax support is that the benefits of SR\&ED extend beyond the performers themselves to other firms and sectors of the economy. The existence of these spillovers or externalities means that, in the absence of government support, firms would perform less SR\&ED than desirable for the economy."

\subsection{Direct vs Indirect Support}

This study examines the direct and indirect measures of support for R\&D and innovation which are the two main types of instruments by which the government usually intervenes to address the market failure issue discussed above. According to the definition given in a recent OECD study (OECD (2010)), direct public funding of $R \& D$ includes grants, loans and procurement while the indirect public funding of $R \& D$ includes tax incentives such as tax credits, tax deductions for $R \& D$, reducing taxes on wages in R\&D and social contributions, and the accelerated amortization of $R \& D$ assets. These two policy instruments have different effects: direct subsidies have the effect of increasing the marginal rate of return on private investment while the tax incentives reduce the marginal cost of R\&D. For the government, the choice of using either of these instruments resides mainly on the comparison of their advantages and disadvantages. Direct measures are often credited with the

\footnotetext{
${ }^{2}$ In 2009 most member States remained far from the national 2010 targets they set for themselves in 2005. In the Europe 2020 Strategy adopted in 2010, the EU maintained the $3 \%$ objective for 2020 and in the following months, member States adopted their 2020 national R\&D intensity targets.
} 
desirable feature that they allow the government to target more precisely the projects it wishes to be made by companies. Thus, in a sense, government can steer R\&D performed by firms in sectors deemed strategic.

Figure 2.3-1: Direct and indirect government funding of business $R \& D$ and tax incentives for $R \& D$, 2007

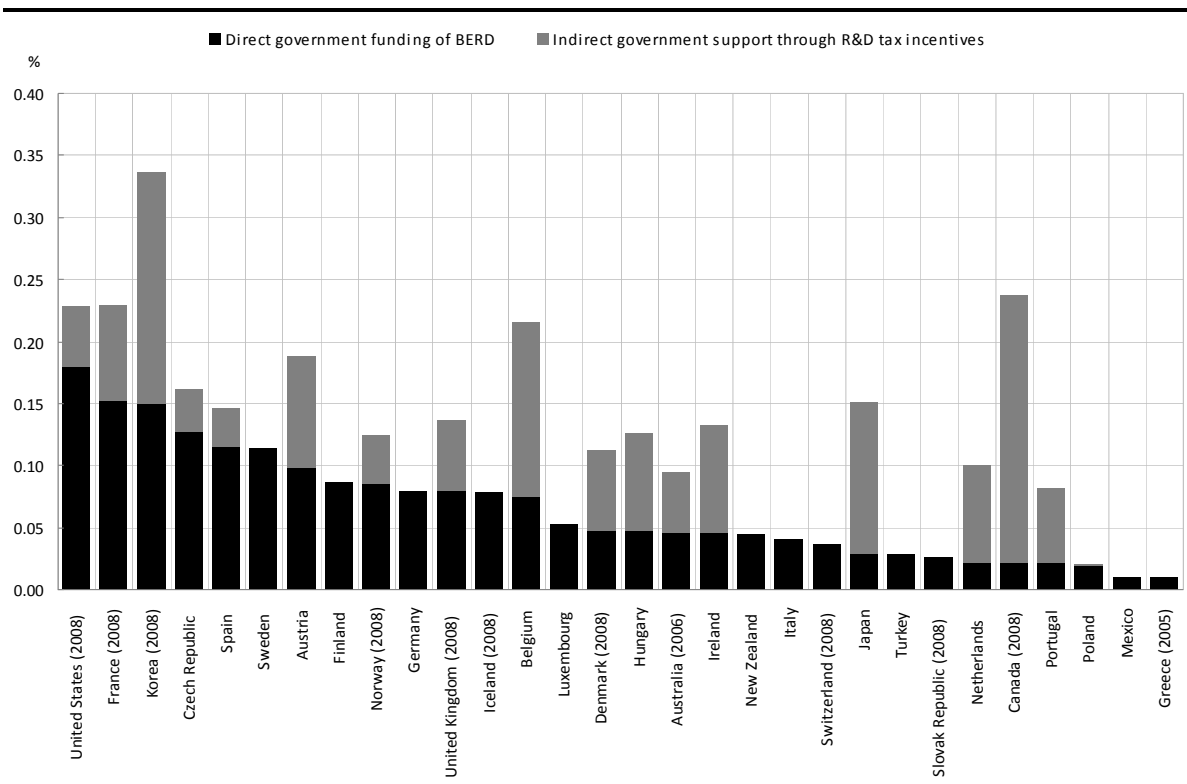

Notes: The R\&D tax expenditures estimates do not cover sub-national R\&D tax incentives. Austria estimate covers the refundable research premium but exclude other R\&D allowances. The United States estimate covers the research tax credit but excludes the expensing of R\&D Italy (volume tax credit of 10\%), Turkey (R\&D tax allowance of 200\%) and Greece (tax credit of 50\% for incremental R\&D) provided R\&D tax incentives in 2007 but the cost of those incentives was not available.

Source: $\quad$ OECD, based on national estimates from the Working Party of National Experts in Science and Technology (NESTI) R\&D tax incentives questionnaire, January 2010; and OECD, Main Science and Technology Indicators Database, December 2009.

In Canada, as in Québec, the policy instrument used by the government is the indirect measures, and this is the case for several decades as will be discussed in more details in next chapter concerning the review of the policies for R\&D and innovation in Québec. This choice is not similar to that of the majority of countries offering R\&D incentives. Indeed, an international comparison of the use of both types of instruments in 2007 (Figure 2.3-1) shows that, out of 30 countries, 19 use more direct measures than indirect and one country (Australia) uses both instruments equally. Of all countries, there are 13 countries that use only direct 
measures. In the group of the 10 countries using indirect measures, Canada is the country whose share of funds spent on indirect measures over direct measures is highest: the amounts spent on indirect measures represented almost 10 times that of direct measures. Then follow Japan and Portugal with amounts of indirect measures 4 and 3 times higher than direct measures. In the remaining 7 countries, this ratio doesn't exceed 2 times. Canada appears as an outlier compared to other countries. The situation should not be different in the case of Québec since it is a major R\&D funder in Canada as it will be shown in the next chapter.

\subsection{Institutional Environment}

One of the key elements of the innovation system of a country is the institutional environment in which evolves innovation business, in particular, the institutional channels through which government incentives are distributed and how the public support programs are administered. In Québec, as in some Canadian provinces, there is the presence of a multitude of provincial and federal bodies that administer support programs which, in some cases, overlap each other without being complementary between them. In addition to direct and indirect support, both the federal and provincial governments also support companies through their agencies by investing funds in the form of equity, capital venture, loans or loan guarantees. For corporations, the consequence is the multiplicity of agencies and rules to deal with for accessing the government support. For example, a small and medium sized enterprise (SME) installed in Québec wishing to benefit from direct and indirect support available may have to deal with at least four bodies: Canada Revenue Agency (fiscal support from the federal government), a federal agency providing direct support (e.g.: National Research Council of Canada (NRC)), Revenu Québec (provincial fiscal support) and a provincial body for direct support such as the Ministère du Développement économique, de I'Innovation et de l'Exportation du Québec (MDEIE). Table 2.4-1 shows the main federal and provincial bodies defining and providing funding to Québec corporations engaged in R\&D and innovation. This situation often forces companies to turn to the services of consultants. Consequently, a private consulting industry increasingly important has developed, offering support and assistance in obtaining government support from both the federal and provincial. The amounts paid by companies to consulting agencies are not well known, but according to some estimates they are significant. These costs reduce the value of the financial support. 
Table 2.4-1: Main federal and provincial bodies funding R\&D and innovation of corporations

\begin{tabular}{|c|c|c|}
\hline FEDERAL & INDIRECT MEASURES & QUÉBEC \\
\hline \multicolumn{3}{|c|}{ Role or nature of assistance } \\
\hline $\begin{array}{l}\text { Department of Finance } \\
\text { Canada }\end{array}$ & $\begin{array}{l}\text { - Responsible for defining the fiscal } \\
\text { policy }\end{array}$ & $\begin{array}{l}\text { - Ministère des Finances du } \\
\text { Québec }\end{array}$ \\
\hline \multirow[t]{2}{*}{ - Canada Revenue Agency } & $\begin{array}{l}\text { Responsible for the administration } \\
\text { of fiscal programs for R\&D }\end{array}$ & - Revenu Québec \\
\hline & $\begin{array}{l}\text { Responsible for the administration } \\
\text { of fiscal incentives for innovation } \\
\text { (on behalf of Revenu Québec) and } \\
\text { interim financing of refundable tax } \\
\text { credits }\end{array}$ & - Investissement Québec \\
\hline \multirow[t]{2}{*}{ FEDERAL } & DIRECT MEASURES & QUÉBEC \\
\hline & Role or nature of assistance & \\
\hline $\begin{array}{l}\text { - Industry Canada (through } \\
\text { NRC) }\end{array}$ & $\begin{array}{l}\text { - Responsible for defining direct } \\
\text { assistance policy } \\
\text { - Direct support }\end{array}$ & $\begin{array}{l}\text { - MDEIE (with support from } \\
\text { the Conseil de la science et } \\
\text { de la technologie }(\mathrm{CST})^{3} \text { ) }\end{array}$ \\
\hline - $\quad \mathrm{NRC}$ & $\begin{array}{l}\text { - Undertaking, assisting or } \\
\text { promoting R\&D throughout Canada } \\
\text { - Direct support (e.g: PARI program) }\end{array}$ & \\
\hline \multirow[t]{4}{*}{$\begin{array}{l}\text { - Industrial Technologies } \\
\text { Office (on behalf of } \\
\text { Industry Canada) }\end{array}$} & - Direct support (e.g: SADI program) & \\
\hline & - Direct support & $\begin{array}{l}\text { - Ministère de l'Agriculture, } \\
\text { des Pêcheries et de } \\
\text { l'Alimentation }\end{array}$ \\
\hline & - Direct support & $\begin{array}{l}\text { - Ministère de l’Éducation, } \\
\text { du Loisir et du Sport }\end{array}$ \\
\hline & - Direct support & $\begin{array}{l}\text { - } \quad \text { Ministères des Ressources } \\
\text { naturelles et de la Faune }\end{array}$ \\
\hline $\begin{array}{l}\text { Business Development } \\
\text { Bank of Canada }\end{array}$ & $\begin{array}{l}\text { - Direct support to venture capital } \\
\text { - Loans }\end{array}$ & \\
\hline \multirow[t]{3}{*}{$\begin{array}{l}\text { - Export Development } \\
\text { Canada }\end{array}$} & - Loans and credit insurance & \\
\hline & $\begin{array}{l}\text { - Loans loans guarantees } \\
\text { - Direct support to venture capital }\end{array}$ & - Investissement Québec \\
\hline & - Direct support to venture capital & - Innovatechs \\
\hline
\end{tabular}

Note: See the list of abbreviations in page 265 .

${ }^{3}$ Since 1 July 2011, the CST was abolished and its activities were transferred to the MDEIE. 


\section{CHAPTER 3}

R\&D and Innovation Policies in
Québec 


\subsection{Introduction}

Profile of Québec's R\&D and innovation policy makes it a low direct subsidy and high fiscal support jurisdiction. This situation is primarily the result of a historical process rather than that of an integrated policy for R\&D and innovation. Indeed, public support for R\&D and innovation has historically developed around the mission of agencies responsible for administering public assistance programs, namely the tax vocation under the auspices of the Ministries of Finance of Canada and Québec, and the vocation of direct support under the responsibility of departments such as Industry Canada (federal government) and the Ministère du Développement économique, de I'Innovation et de l'Exportation du Québec (provincial government) through their funding agencies in particular. It is trivial to understand that the presence of several provincial and federal actors offering a multitude of programs to support R\&D and innovation has not favored the establishment of a R\&D-innovation and federal-provincial built policy, quite the contrary. Indeed, in recent decades, while the federal government, like other OECD countries, continued to consolidate and increase its activities in R\&D and innovation in the provinces, the provincial governments, notably the Government of Québec, followed the same path but often separately. Thus, today there is in the Canadian provinces a superposition of federal and provincial measures targeting sometimes the same sectors. In this respect, Trépanier (1992) (p. 128) writes:

"In Québec, the scientists are in some way, faced with two policies: the Government of Québec and the federal government. Each level of government has its own science and technology policy and its own agencies to define and implement programs to support the scientific and technical activity. This duplication also applies to the selection of priority areas for R\&D: each level of government establishes its own project lists sectors, which are never exactly the same".

That said, it is noteworthy that the heterogeneity or the diversity of policies do not always create a sub-optimal situation as evidenced by studies conducted in other jurisdictions. For example, in a review of the U.S. government technology policies, Alic, Mowery and Rubin (2003) note that although the United States has never had a coherent set of innovation policies, government actions have profoundly influenced the rate and direction of technological change since the founding of the republic. They go on to state that despite the heterogeneity in federal policies - or perhaps because of it, given the high levels of uncertainty that characterize innovation-government actions have been remarkably effective.

What can we say about the situation in Québec despite several decades of heterogeneity and active federal and provincial policies? What are the incentives 
that have been and continue to be offered to Québec businesses by both levels of government? How do the incentives offered in Québec compare to those of the other Canadian provinces? What are the costs of these incentives? What are the results in terms of overall $R \& D$ investment growth in comparison to the result obtained in other jurisdictions or in terms of reducing the productivity lag of Québec? These are among many questions for which an analysis of indicators and other descriptive statistics is useful before addressing in-depth econometric studies. This is one of the objectives of this chapter.

To better understand the current profile of Québec, we start this chapter by a historical overview of the major developments concerning the policies pursued by the federal and provincial governments with regard to direct and indirect incentives in the last decades (Section 3.2). The emphasis is primarily on the policy of the Government of Québec since it is more specific to Québec companies. In the following section (Section 3.3), we present the description of the Québec government's support measures of R\&D and innovation in recent years. Because of the lack of a coordinated federal-provincial policy, the presence of a multitude of programs and the lack of information about some of these programs, it is quite impossible to draw up a complete and accurate taxonomy of support measures to $R \& D$ and innovation in Québec. Consequently, we merely classify them according to their nature (indirect or direct). Estimates of the costs of the different measures are also presented. In Section 3.4, we do the same exercise with the Canadian government's support measures by presenting indirect measures first followed by direct measures. In Section 3.5, we describe the net cost of the combined federalprovincial indirect incentive. We then present in the last three sections of this chapter the statistics on the state of R\&D and innovation in Québec, with the aim to draw lessons on the success of the policies presented in the preceding sections. More specifically, Section 3.6 presents a comparison of the R\&D measures offered in each Canadian province and by the federal government, Section 3.7 presents the main statistics on R\&D and highlights the place of Québec in the world, and lastly, in the light of the statistics previously presented, Section 3.8 discusses the Québec's productivity gap issue.

\subsection{Historical Overview of R\&D and Innovation Policies in Québec}

The history of science policy of Québec can be divided into two periods. The first period that goes from the 1960s to the beginning of the 1980s during which the Government of Québec sets up its own scientific policy as well as the public institutions for research and research councils that act as advisors on major policy orientations in key areas or as research granting agencies. The second period that 
began in the 1980s is that of the implementation and strengthening of Québec's fiscal policy as the main instrument of policy to support R\&D and innovation. Appendix 3.A presents key highlights of the major developments that affected the science and technology policies in Québec since the 1960s.

\subsubsection{From the 1960 s to the beginning of the $1980 \mathrm{~s}$}

During the 60s and 70s, the Government of Québec had not yet defined its strategy to support companies engaged in research and innovation and there were no direct subsidy programs for R\&D performed in companies. Public support for R\&D and innovation for companies in Québec came almost exclusively from the federal government. Indeed, the later offered a wide range of direct support measures, including special programs to support innovation, contracts and loans from the Industrial Development Bank $(I D B)^{4}$ or the intramural R\&D performed in government laboratories for the benefit of enterprises. Among the special programs to support innovation, one can cite mainly the Program for Advancement of Industrial Technology (PAIT), the Industrial research and development incentives act (IRDIA), the Defence Industry Productivity Program (DIP), the Defence Industrial Research Program (DIRP) of the Defence Research Board (DRB) and the Industrial Research Assistance Program of the National Research Council of Canada.

During this period, the Government of Québec started the establishment of its agencies of intervention in key areas. It's the case in 1962 with the creation of the the Société générale de financement (SGF) and in 1964 of the Medical Research Council of Québec which is the first provincial agency of research funding. In the same vein, the governement also created in 1969 two major research organizations, namely the Centre de recherche industrielle du Québec (CRIQ) and the Institut national de la recherche scientifique (INRS), affiliated to the University of Québec created just one year earlier, in 1968. In the turn of the 1960s and in the 1970s, other provincial agencies of intervention have been created, including the Société de développement industriel (SDI), the Société SOQUIP (Société québecoise d'initiative pétrolière) and REXFOR (Société de récupération, d'exploitation et de développement forestiers du Québec). Through these agencies, the Government of Québec increasingly supported industry towards the end of the 1970s, but its subsidies were granted primarily through collaborations between industry and government research laboratories and university research centres. This vision was mainly symbolized by the CRIQ which was the main body of government intervention through its mission, that is, promoting and carrying out in its own laboratory the development of industrial innovations. Thus, by engaging itself in research and development and making the output available to the industry, the

\footnotetext{
${ }^{4}$ The Industrial Development Bank is the ancestor of the Business Development Bank of Canada (BDC).
} 
government hoped to stimulate industrial growth. This approach that was also experimented in other jurisdictions lasted until the late 70 s.

Concerning the indirect measures, things were not different from the situation described above with respect to direct measures, i.e. R\&D and innovation support came primarily from federal programs. This is also the case in other Canadian provinces: the Government of Canada was the only one to offer fiscal incentive for $R \& D$ across the country. Businesses located in Québec and in other provinces could benefit from some traditional tax measures such as the deduction and the tax credit that were in place since the 40s. From 1977, the Government of Canada introduced the SR\&ED tax credit, a non-refundable tax credit with a base rate varying between 5 and $10 \%$ of current and capital expenditure on R\&D, according to company size. In addition to this credit, the Government of Canada also introduced two tax measures between 1977 and 1978. In these cases, the novelty was that firms performing R\&D activities in Québec could also receive the same tax benefit in their provincial income tax. These tax measures consisted mainly of:

- an immediate tax deduction of current expenses and capital devoted to $R \& D$, the year they are incurred. This deduction was available to both individuals and corporations;

- an additional deduction of 50 percent of current expenditures and R\&D capital that exceeded the average of the last three years. This deduction was only available to companies.

\subsubsection{Since the beginning of the 1980s}

During the federal Budget Speech of 19 April 1983, the Finance Minister of Canada announced the abolition of the additional deduction of 50 percent in response to noticed abuses. To this end, he filed a consultation document entitled "Tax policy in research and development" in which he proposed to improve the non-refundable tax credit by increasing its base rate from 10 to $20 \%$. Following this proposal, the Government of Québec decided in the Budget Speech of May 10, 1983 to remove the additional deduction of 50 percent as suggested by the federal Minister of Finance. However, instead of establishing the non-refundable tax credit of $20 \%$ suggested, the Government of Québec rather established a tax credit of $10 \%$ on salaries paid in Québec under the R\&D expenditures. Unlike the federal tax credit, the new provincial credit is refundable. This is the first time the Government of Québec established a tax measure to encourage scientific research and experimental development. It is, moreover, the first time as well that a Canadian province introduced a fiscal measure aiming to support R\&D. It is important to note that this decision was taken in a context where the Governement of Québec advocated the need for a technological shift in response to the global recession and, 
as such, he introduced an economic action plan over four years to support this technological change ${ }^{5}$.

Even though the implementation of this tax credit in 1983 allowed the introduction in Québec of indirect measures as policy instrument, the tax incentives have become the main and preferred instrument of policy for R\&D and innovation of the Government of Québec only four years after that date, namely in 1987. Thus, the government announced in the Budget Speech of April 30, 1987 its intention to stimulate job creation through increased support to the innovation process and also its willingness to increase the ratio of R\&D expenditures to GDP to the average performance of $\mathrm{G7}$ countries. For this purpose, four new measures were announced: First, an enhancement of the support to human capital in businesses, including an increase from 10 to $20 \%$ of the refundable tax credit for R\&D salaries. In addition, to attract foreign researchers of high caliber, a tax holiday from the tax on personal income was also introduced. Second, the tax credit of $20 \%$ for R\&D salaries as part of cooperation projects between universities and businesses was increased to $40 \%$ and extended to all R\&D expenditures in universities. Third, support to facilitate the raising of venture capital to finance $R \& D$ was made available, including a tax benefit for individuals providing the venture capital by investing in research and development. Specifically, this tax benefit consisted of an additional deduction of $331 / 3 \%$ with respect to the total R\&D expenditures in Québec, which was added to the deduction up to $100 \%$. Finally, these tax measures were complemented by increased direct funding to universities to enhance their research capacity. For the 1987-1988 financial year only, the additional amounts intended to increase the base funding, reduce deficits and pay their expenses for the purchase of equipment totaled $\$ 84$ million.

In 1988, the Government of Québec held a summit on technology. On the occasion of the opening of the summit, Prime Minister of Québec, Robert Bourassa, reiterated Québec's choice to focus on fiscal measures rather than subsidy programs by stating:

"We believe that fiscal approach is better suited to business needs, and this, mainly for four reasons:

\footnotetext{
${ }^{5}$ In the early 80s, following the global economic recession, the government advocated the need for a technological shift in the fight against unemployment resulting from the recession. The objective of technological change was, in a global context of increasing trade liberalization, to make Québec products competitive in terms of quality and price and this, by fostering businesses growth, by developing skills and building on new areas such as new electronic technologies and biotechnology. In this respect, the government implemented in 1982 a four-year economic program comprising new measures to support technological change. These were published in a paper entitled: Technological change: Building Québec Phase 2. Economic Action Program 1982-1986. At the same time, the government took a number of measures, including the adoption of the Act to Promote Scientific and Technological Development, the creation of the Fonds FCAR and the FRSQ, and the transformation of the Conseil de la Politique Scientifique (CPS), the provincial structure established in 1971 to advise scientific policy, in the Conseil de la Science et de la Technologie (CST).
} 
The first is that Québec's tax credits are refundable so they give the same monetary benefits as grants without the drawbacks.

The second is the fact that companies prefer to take advantage of tax breaks rather than subsidies for obvious reasons of simplicity and confidentiality.

The third is to the effect that the tool of taxation minimizes bureaucratic controls that are inevitable when government assistance is provided through programs.

The fourth is that tax credits do not include administrative costs and, thus, allow for every public dollar spent on research and development, transfer to the company an amount of assistance greater than when it comes to subsidies as part of a program."

As we will see in the following sections concerning the review of direct and indirect measures available to businesses in Québec, the policy pursued by the Government of Québec since then until today has not really changed: the government has maintained and even strengthened the strategy of using generic fiscal measures as the main tool for public intervention.

\subsection{Support Measures of the Government of Québec}

\subsubsection{Indirect Support Measures}

As mentioned previously, indirect support measures for R\&D activities in Québec materialize principally in the form of refundable tax credits. Hence, over the past decades, several refundable tax credits targeted to firms have been implemented in Québec and some of them have been withdrawn already. However, the most important refundable tax credit ever launched, that is, the SR\&ED tax credit that was introduced since 1983 is still in place. Furthermore, this refundable tax credit has been improved along time. In order to maintain the competitiveness of its tax system compared to other Canadian provinces or to cover a specific area or sector improperly covered by the generic support provided by the SR\&ED tax credit, the Government of Québec also introduced measures other than refundable tax credits such as superdeductions for R\&D that allowed special R\&D expenditure deductions to firms in the calculation of their taxable income. We present below the main fiscal measures that took place in Québec in the last three decades, namely, the SR\&DE refundable tax credit; the superdeductions for $R \& D$ and the refundable tax credit based on the increase in R\&D expenditures. 


\subsubsection{The refundable Tax Credit for Scientific Research and Experimental Development}

This tax credit is the main R\&D fiscal incentive in Québec. Introduced in the Budget speech of March 1983, it was the first fiscal measure aimed at supporting R\&D in Québec. As reveals Table 3.B-4, the SR\&ED has evolved a lot through time. At its introduction this tax credit was essentially based on the salaries of researchers, but it has been greatly enhanced along time. In addition to human capital, the Government of Québec put the emphasis on both human capital and more intense cooperation between businesses, universities and research centres in designing the tax credit. This has lead to the following four measures:

\section{Fiscal measures targeted to human capital:}

\section{a. The Refundable Tax Credit for Salaries and Wages of Researchers}

As noted above, this tax credit was the sole component of the SR\&ED at its introduction in June 2003 and it still accounts as its major component with a proportion of more than $95 \%$ in the last years for overall fiscal incentives in Québec. The tax credit can be claimed by corporations (or taxpayers in general) that conduct R\&D activities in Québec themselves, or that have such activities conducted in Québec on their behalf.

As of December 5, 2006, the incentive provided by Revenu Québec permits taxpayers that are corporations to claim a refundable tax credit on the salaries and wages $^{6}$ they pay in Québec in a given taxation year that amount to:

- $37.5 \%$ in case of $\mathrm{SMEs}^{7}$;

- between $37.5 \%$ and $17.5 \%$, in the case of corporations with assets between $\$ 50$ and $\$ 75$ million. A linear reduction in the rate of the tax credit (from $37.5 \%$ to $17.5 \%$ ) is applied with respect to asset increase as follows:

$37.5 \%-\{[(\mathrm{A}-50000000 \$ \times 17.5 \%] / 50000000 \$\}$, with $A$ being corporation asset;

- $17.5 \%$, in the case of corporations with assets of $\$ 75$ million or more.

The rate applicable to qualified expenditures that exceed the first \$2 million is $17.5 \%$, regardless of the date of the expenditures or the amount of the corporation's assets.

\footnotetext{
${ }^{6}$ The tax credit is calculated on the full amount of salaries and wages paid to employees who devote at least $90 \%$ of their time to R\&D activities or support. The qualifying amount paid to employees devoting less than $90 \%$ of their time to R\&D activities is calculated according to the time spent by each employee on such activities.

${ }^{7}$ Québec's Taxation Act defines a corporation as an SME, a business with asset, including those of associated corporation, less than 50 million. In addition, the business must not be controlled by one or more persons not residents in Canada.
} 
Taxpayers other other than corporations may claim a refundable tax credit that amount to $17.5 \%$.

The evolution of the rates and rules that applied to this tax credit can be found in Table 3.B-4.

\section{Fiscal measures targeted to R\&D expenditures}

b. The Refundable Tax Credit for University Research or Research Carried Out by a Public Research Centre or a Research Consortium

This tax credit permits firms that conclude a university research contract with an eligible university entity, a public research centre or a research consortium to claim a refundable credit of $35 \%$ of the eligible R\&D expenditures. In the case where the research is done by an eligible university entity, a public research centre or a research consortium that is not affiliated to the taxpayer, $20 \%$ is subtracted from the eligible expenditures in calculating the tax credit (the credit is calculated on $80 \%$ of qualified expenditures). However, entitlement to the tax credit for each contract entered into need to be authorized by an advance ruling from Revenu Québec before any amounts provided for in the contract are paid.

\section{c. The Refundable Tax Credit for for private partnerships Pre-competitive Research}

The Québec tax credit for private partnerships pre-competitive research replaced the refundable tax credit for pre-competitive research since March $23^{\text {th }}, 2006$. Under this scheme, a corporation that enter into a cooperative agreement with non affiliated corporations to carry out work themselves, or to have work carry out on their behalf, under a pre-competitive research project, may claim a refundable tax credit of $35 \%$ of the eligible R\&D expenditures incurred in Québec. As in the case above, to be valid for a refundable tax credit, the cooperative agreement needs to be authorized by the government.

\section{d. Tax Credit for Fees or Dues Paid to a Research Consortium}

A corporation that is a member of a research consortium may claim a refundable tax credit of $35 \%$ of its total fees or dues paid to a research consortium to conduct $R \& D$ in Québec related to its activities. The research consortium must be recognized by the government in order to be entitled to the tax credit.

A summary of the Québec's SR\&ED tax credit is given in Table 3.B-1, while Table 3.B-4 highlights the main changes to this tax credit from its introduction in 1983 to 2010.

\subsubsection{The Superdeductions for R\&D (1999-2000)}

Introduced in the Budget speech of March 1999 and withdrawn a year later, the superdeductions for R\&D were an alternative to the refundable SR\&ED tax credits. 
Under this scheme, firms could deduct from their taxable income labour expenditures associated with the R\&D conducted by a company and expenditures related to research contracts concluded with third parties according to the rates shown in Table 3.3-1.

Table 3.3-1: $\quad$ Rates applicable to superdeductions

\begin{tabular}{lcc}
\hline Activities & SME & Large corporations \\
\hline In house R\&D and certain outside contracts $^{\mathrm{a}}$ & $460 \%$ & $230 \%$ \\
R\&D carried out by another entity $^{\mathrm{b}}$ & $460 \%$ & $460 \%$ \\
\hline
\end{tabular}

a) For companies which conduct R\&D in house and whose assets are between $\$ 25$ million and $\$ 50$ million, the $460 \%$ rate is reduced linearly to $230 \%$.

b) University research centres, public research centres, research consortia or business combinations conducting or contracting out pre-competitive research.

Source: Ministère des Finances du Québec, Budget 1999-2000, Additional Information on the Budgetary Measures.

From the perspective of the Government of Québec, this measure was equivalent to its own SR\&DE tax credit described above since the tax benefit to an SME applying the $460 \%$ rate of the superdeduction was similar to the amount it would have had if a tax credit rate of $40 \%$ had applied. In reality, firms choosing the superdeductions reduced substantially their net investment cost far below the investment cost in the case of refundable tax credit. The reason for this is that, the reduction of the cost of $R \& D$ arising from the superdeductions was greater than that from the SR\&ED tax credit due to the differential in the fiscal treatment applied by the federal government to both measures. For example, the net cost incurred by an SME for a $\$ 100$ wage expenditure would be only $\$ 9$ in the case of superdeductions compared to $\$ 27$ if a tax credit were claimed (see Section 7.2.2 for additional details on the mechanism of superdeductions).

It should be noted that when the superdeductions were introduced, firms still had the option to choose between the refundable tax credit and the superdeductions so that, in practice, firms with sufficient income were better off choosing superdeductions in order to reduce their eligible business income to zero and those with an operating loss were better off choosing the refundable tax credits. The superdeductions lasted only a fiscal year, but as one can expect, they were very popular with firms. The Government of Québec, however, announced its withdrawal after the decision of the federal government to apply the same rules in the treatment between refundable tax credits and superdeductions for R\&D (more on superdeductions in Chapter 7 devoted to the evaluation of this program). 


\subsubsection{The Refundable Tax Credit Based on the Increase in R\&D Expenditures (1999-2004)}

The Government of Québec announced this tax credit along with the superdeductions in the Budget speech of March 1999. It was implemented for a five year period as part of the effort to increase the ratio of R\&D expenditures to GDP to the average performance of G7 countries. The credit applied only to SMEs, i.e. companies with assets under $\$ 25$ million. Under this tax credit, SMEs could claim an additional $15 \%$ tax credit on the eligible $R \& D$ expenditures exceeding a reference amount equal to the average R\&D expenditures for the preceding three years. SMEs that opted for superdeductions could also benefit from this tax credit with an additional deduction of $190 \%$ of their eligible R\&D expenditures.

\subsubsection{Fiscal Measures Targeting other Innovation Activities}

In the late 1990s and early 2000s, the Government of Québec implemented several indirect measures to stimulate the expansion of innovation projects, mainly in sectors related to the knowledge-based economy such as biotechnologies or technology information and communications sectors. Among these, one can cite the following six main fiscal measures, all administered by Investissement Québec, relating to the carrying out of activities in certain designated sites, i.e. measures relating to information technology development centres (CDTIs), the Cité du multimédia de Montréal (CMM), the Centre national des nouvelles technologies de Québec (CNNTQ), the new economy centres (CNEs), E-Commerce Place (CCE) and the biotechnology development centres (CDBs). Of these measures, only the last, the tax credit for the CDB continues to offer financial assistance. The first five measures were abolished by the 2003-2004 Budget Speech of June 12, 2003. However, the government decided to allow companies who benefited from these measures before that date to continue to claim the tax credits granted as prescribed before June 12,2003 , provided for these companies to have obtained, before June 12, 2003, an eligibility certificate issued by Investissement Québec.

In general, the nature of assistance provided by these measures mirrors that of the tax credit for research and development in that, the terms of these measures follow the same principle: financial support is primarily granted for human capital. Indeed, for most of these measures, the tax assistance takes the form of a refundable tax credit of up to $40 \%$ calculated on the salary paid to a specified employee. Additional benefits to the corporation carrying out an innovative project in a designated site enable the latter to receive refundable tax credits for the acquisition or leasing of eligible specialized equipment. Such a corporation is also entitled to receive non $R \& D$ advantage such as a five-year tax holiday regarding income tax, the tax on capital and a refundable credit of the employer contribution to the Health Services Fund (HSF). It should be noted however that according to the 
usual rules regarding the tax credit refundable, the amount of wages must be reduced by the amount of any government assistance and any profit or advantage attributable to those wages.

One limitation to the scope of such measures is that they target only companies based on designated geographic locations. In other words, the tax assistance granted by these measures was not or is not available throughout the province of Québec.

The estimated costs of all indirect measures for innovation of the Government of Québec from 1997 to 2007 are presented in the next section along with the costs of indirect measures for R\&D. The cost of each measure, for the major ones, is presented in Table 3.C-5. Finally, Table 3.B-3 provides a summary of the activities covered by the measures mentioned above and the nature of the aid paid.

\subsubsection{Costs of Indirect Measures for the Governement of Québec}

The costs of tax incentives for R\&D and innovation have historically experienced a significant increase due to the policy adopted by the Government of Québec to use fiscal measures as the main policy tool to support R\&D and innovation. Table 3.C-5 provides an estimate of costs associated with each tax measure during the period from 1997 to 2007. It thus includes, for each year, an estimate of the total tax support for R\&D and innovation of the Government of Québec. As shown in Figure 3.3-1, over a period of ten years, the cost almost tripled, going from just under $\$ 350$ million in 1997 to over a billion dollars in 2007. The majority of these amounts was allocated to fiscal measures for R\&D, which was predominated by the component "salaries of researchers" of the SR\&ED tax Credit. Indeed, as can be seen in Table 3.C-5, this tax credit has been the most used by companies, totaling alone around 90 percent of the total amounts allocated to the tax support for R\&D in Québec. This popularity is due to the favorable terms offered by the tax credit (see Section 3.3.1.1). We did not find any other Canadian or foreign jurisdiction providing such tax benefit (a comparative summary of the tax systems of the Canadian provinces is given in Table 3.B-4). This means that the fiscal policy of the Government of Québec in respect of R\&D targets primarily human capital rather than on overall R\&D expenditures as is the case for Canadian tax policy. 
Figure 3.3-1: Evolution of the estimated cost of fiscal incentives for R\&D and innovation, 1997-2007

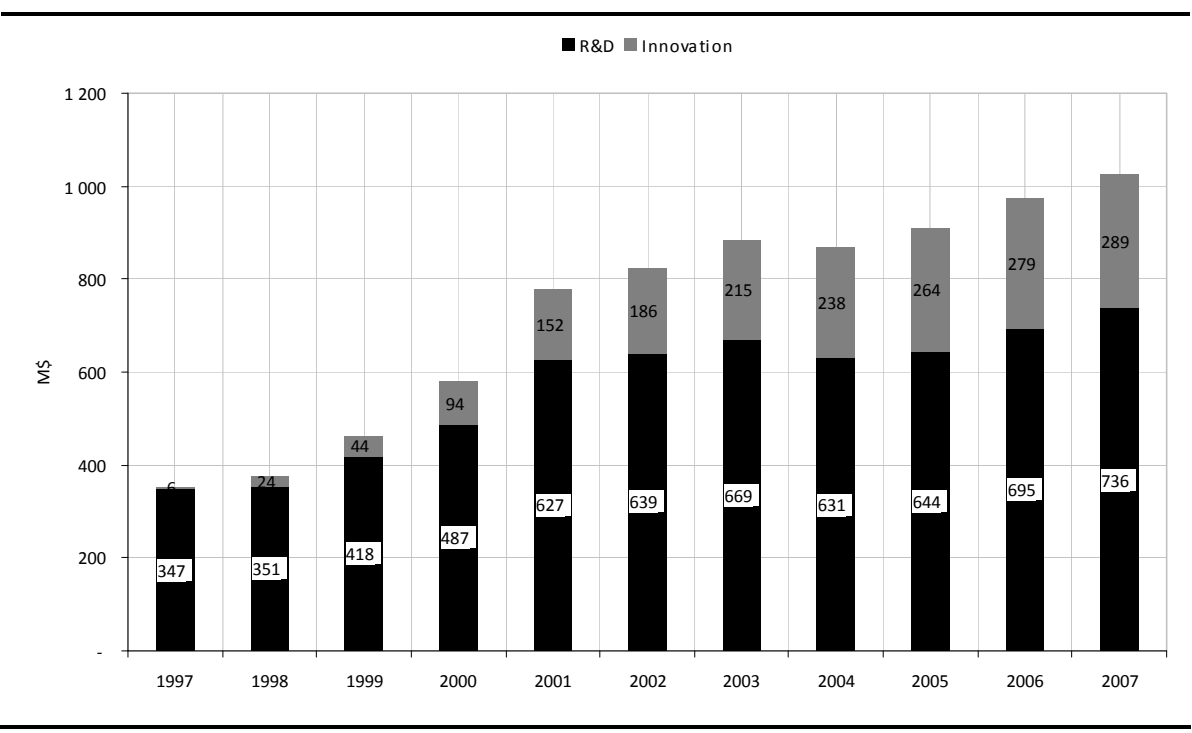

Source: Ministère des Finances du Québec, Tax expenditures, 2005, 2008, 2009 and 2010 Editions.

Despite the predominance of the tax credits for $R \& D$, we note that the fiscal measures to support innovation activities experienced the largest growth over the past decade. In 1997, the share of the costs of these measures in the total tax Budget for R\&D and innovation did not exceed 2\%. In 2007, this proportion increased to over $30 \%$. The introduction of several new tax measures to support innovation initiatives is the main reason for this increase.

\subsubsection{Direct Support Measures}

Historically, direct subsidies to R\&D and innovation of the Government of Québec were set up according to priority sectors defined by the government. Thus, the administration of these programs has been delegated to departments and agencies responsible for these sectors. In recent decades several programs have been implemented by these bodies and, unlike the tax credit program for SR\&ED whose terms are defined in the law, these programs were often temporary. The MDEIE is the lead ministry of the Government of Québec that provides direct support to enterprises. It also provides financial support to $\mathrm{CCTT}^{8}$ which are college centers for

\footnotetext{
${ }^{8}$ First introduced in 1983, the mandate of CCTTs is to exercise in a particular area of applied research, technical assistance to the company and information to assist in the development and implementation of technological innovation projects, to implantation of new technologies and their dissemination. These centers intervene in various stages of a process of change in technology: technology transfer, research-
} 
technology transfer from public research to the industry. Other ministries such as the MAPAQ, MELS or MRNF also provide financial support to enterprises through their program for technological innovation support, but more modestly.

More recently, the MDEIE has implemented a number of programs following the adoption of the Québec strategy for research and innovation (SQRI) in 2006 and the Action plan of for the manufacturing sector in 2007. The most important of these programs are the Program for the Support of R\&D and the Program for the Support of innovation described below.

\subsubsection{Program for the Support of Research (PSR)}

This program aims to support research and innovation projects or initiatives. The assistance covers three components: the first, PSR 1 aims to promote structuring initiatives to support the development of research, PSR 2 aims at supporting the modernization, acquisition and development of infrastructure research, and PSR 3 has the goal to increase the participation of companies and strategic alliances in international consortia of research and innovation. It aims particularly at non-profit organizations involved in the different areas of research, innovation and diffusion of science and technology, research institutions or public health networks and the education and business. In the latter case, the target companies in priority are those with fewer than 250 employees or with assets not exceeding $\$ 50$ million, and the projects concerned are those of the PSR 3. Here are, briefly, details of these three components:

For PSR 1, the eligible project are initiatives to support the development of research such as, projects or activities for the development of research in industries, sectors or the development of economic niche, social and cultural projects to promote the creation, development or strengthening of partnerships or research networks involving academic, institutional and industrial projects.

For PSR 2, eligible projects concern the repair, expansion, renovation and construction of infrastructure and the acquisition of structural facilities required for research, innovation, promotion and dissemination of research with an eligible expenditure of at least $\$ 100000$. The financial assistance is in the form of nonrefundable contribution of up to $80 \%$ of eligible expenses. However, the combination of government assistance (federal, provincial and municipal) may not exceed $80 \%$, excluding contributions to the functioning of the body. The contribution of the sector to the project must be at least $20 \%$.

Finally, for PSR 3, financial aid varies depending on the type of project and does not exceed $50 \%$ of eligible expenses related to the achievement of all project activities in the Québec portion. As for the eligible expenses, the following expenses

oriented design of a new product or a new production process, technical assistance to facilitate the implementation of changes in the company, etc. 
are allowed: the cost of living and traveling abroad Québec researchers, student scholarships, salary costs of R\&D personnel involved in the project (depending on the type organization), the fees of consultants and subcontractors; the rental or purchase of new equipment useful to the project consumables, the costs related to the exploitation of research and innovation results, and protection of intellectual property.

\subsubsection{Program for the Support of Innovation (PAI)}

This program is intended to support technological innovation in enterprises. The program is open to legally constituted companies, including cooperatives and social economy enterprises, operating in Québec. It aims, in particular, companies of 200 or fewer employees. Financial assistance takes the form of a non-repayable contribution of up to $40 \%$ of eligible expenses. These include professional fees for specialized services, the direct costs of labor assigned to the project, direct costs (equipment, materials and inventory, moving), and charges for obtaining the intellectual property or rights to acquire or license intellectual property.

However, capital expenditures and expenses eligible for a refundable tax credit are excluded. The total financial assistance for a project is limited to a maximum of $\$ 400000$, including a limit of $\$ 100000$ to complete the studies necessary for project planning or development of a marketing plan.

\subsubsection{Costs of Direct Programs for the Government of Québec}

The effort of the Government of Québec for the programs of direct support to R\&D and innovation is significant. Table 3.C- 6 shows the evolution of the Cost of R\&D and Innovation programs in Québec from fiscal years 2000-2001 to 2003-2004. In 2003-2004, total spending for direct support programs to R\&D and innovation totaled $\$ 572$ million. However, the bulk of this funding goes to the government priority sectors, whose mandate is entrusted mainly to universities and colleges, hospitals and its own research organizations like the CRIQ.

Thus, concerning direct assistance programs for R\&D, the sector of high education, comprising colleges and universities, with about $\$ 377$ million, is the sector that received the largest share of funds allocated to direct support programs in 2003-2004 (Figure 3.3-2). This amount represented $76 \%$ of the money allocated by the Government of Québec to direct subsidy programs.

The other sectors received respectively: hospitals and health agencies (7\%), enterprises (7\%) and research agencies of the Government of Québec (1\%). The remaining, about $9 \%$, was paid to other organizations not included in the above categories. 
Figure 3.3-2: Distribution of the costs of direct programs for R\&D, by sectors, 2003-2004

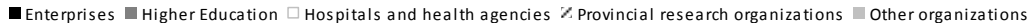

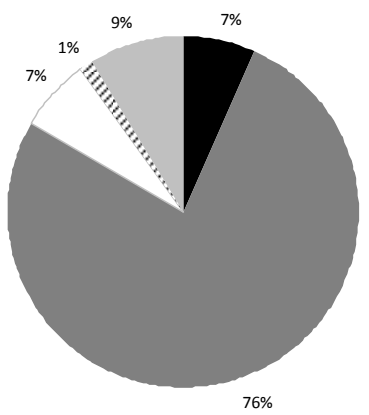

Figure 3.3-3: Distribution of the costs of direct programs for innovation, by sectors, 2003-2004

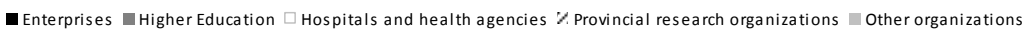

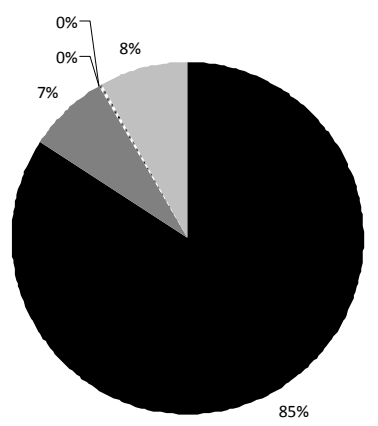

Source: Institut de la statistique du Québec, Survey of research, science, technology and innovation expenditures in the Québec public administration (1999-2000 to 2003-2004).

With regard to assistance programs for innovation, the same pattern is observed as in the case described above: $\$ 79.5$ million (85\%) were allocated to direct support programs for innovation against \$252 million (18\%) for tax programs.

As we described in Section 3.2, this distribution reflects the choice of the Government of Québec and confirms its profile as a high tax incentives and low subsidies jurisdiction. 


\subsubsection{Total Costs for the Government of Québec}

The addition of the total cost of direct and indirect measures provides an overview of the distribution of expenditure of the Government of Québec for its support to $R \& D$ and innovation. As shown in Figure 3.3-4, for the base year 2003-2004, it appears that tax expenditures, composed almost entirely of tax credits, are the largest spending with more than $60 \%$ of the total amount allocated to support R\&D and innovation.

Figure 3.3-4: Distribution of the costs of $R \& D$ and innovation programs, total direct and indirect measures, 2003-2004

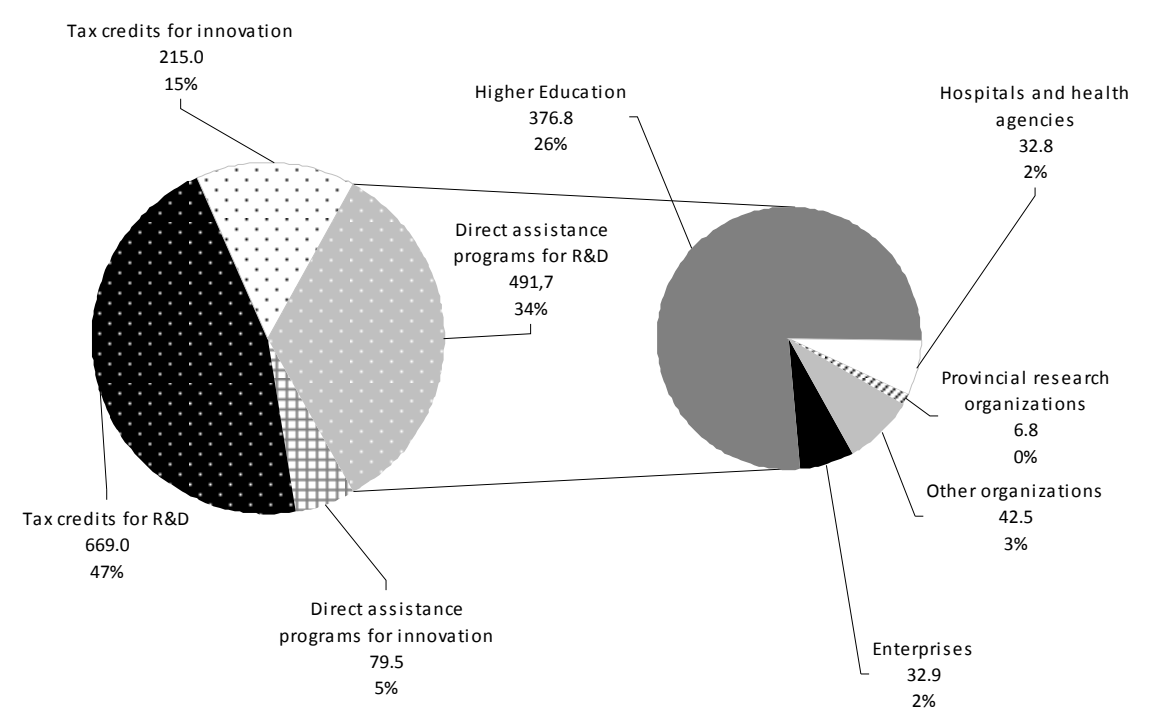

Source: Institut de la satatistique du Québec, Survey of research, science, technology and innovation expenditures in the Québec public administration; Ministère des Finances du Québec, Tax expenditures, 2005 Edition.

The comparison of the Québec government's effort in R\&D with that of other Canadian provinces shows that the former has grown tremendously over the years. Over the period in which we conducted our work, from 1997 to 2004, the total spending in R\&D of the Government of Québec has more than doubled, from just more than $\$ 200$ million over a half-billion. Québec has maintained its second place ranking after the province of Ontario between 1994 and 2004 in terms of absolute spending and spending ratio to GDP. 
Figure 3.3-5: Comparative evolution of the total R\&D expenditures of Canadian provincial governments, 1994-1995 to 2002-2003

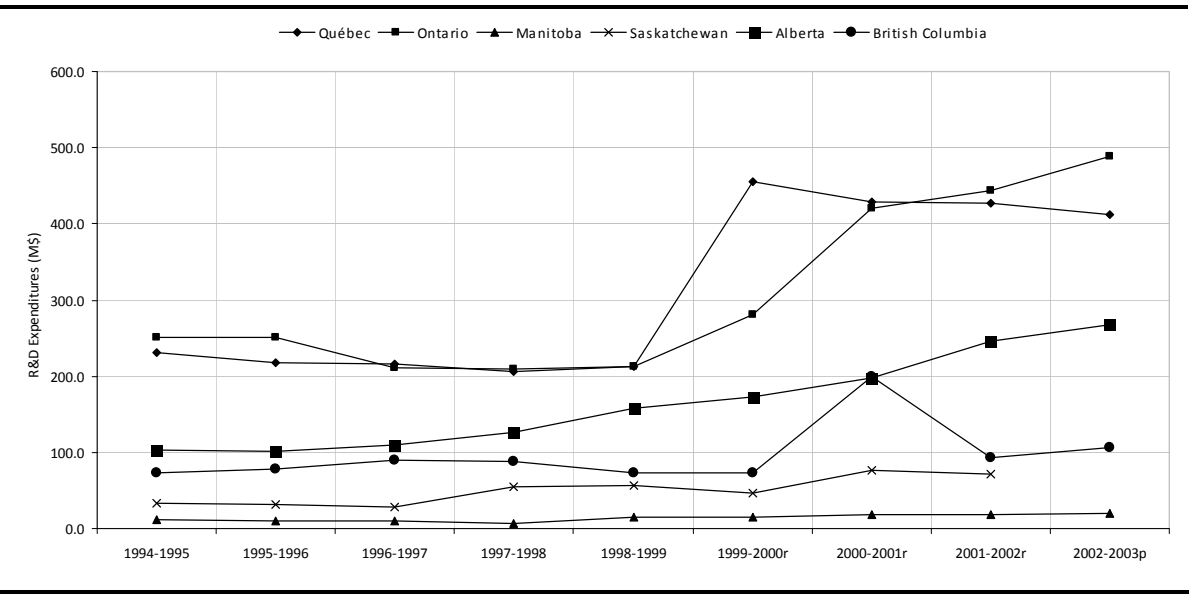

Source: Statistics Canada, Scientific and technological (S\&T) activities of provincial governments, 199495 to 2002-03, 88-001-x2004006, June 2004.

\subsection{Support Measures of the Federal Government}

\subsubsection{Indirect Support Measures}

The Canadian SR\&ED tax credit is the oldest and largest tax credit aimed to support $R \& D$ introduced under this name in 1977 by the federal government. However, the history of fiscal support for R\&D by the federal government goes back much further in time, especially during the 40s. In a report dating from 1989 (updated in 2006), Odette Madore, of the Economics Division, Library of Parliament of Canada, presents a historical review of tax incentives the Government of Canada, divided over three periods:

- From 1944 to 1986: establishment of traditional tax measures such as the deduction and the tax credit, together with some additional tax measures found wanting after testing.

- From 1987 to 1994: refinement of the deduction and the tax credit for ease of use and improve administration.

- Since 1995: broadening and facilitating access to the SR\&ED tax system.

In its form in 1977, the SR\&ED tax credit allowed businesses a tax credit varying between 5 and $10 \%$ of current expenditure and capital, according to company size. Today, the tax base of the SR\&ED tax credit of is much broader and covers more taxpayers than at the beginning (for historical review of the main changes to the federal tax see Madore (1989)). The expenses eligible for the tax credit are eligible 
current and capital expenditures in respect of SR\&ED in Canada performed by, or on behalf of, a taxpayer and related to a business of the taxpayer.

Since the Federal Budget Speech of 2009, the incentive provided by Revenue Canada permits:

- Small Canadian-controlled private corporations (CCPCS) -i.e. with prioryear taxable income of up to $\$ 500,000$ (increased from $\$ 300,000$ in Budget 2006 , and from $\$ 400,000$ in Budget 2009) and taxable capital of up to $\$ 10$ million - to claim a refundable investment tax credit (ITC) of 35\% of qualifying current and capital SR\&ED expenditures, to a maximum of $\$ 3$ million of expenditures per year. Over the \$3 million SR\&ED expenditure limit, the credit rate is reduced to $20 \%$, of which $40 \%$ may be refundable.

- Large CCPCs with prior-year taxable income between $\$ 500,000$ and $\$ 800,000$ or with prior-year taxable capital employed in Canada between $\$ 10$ million and $\$ 50$ million to claim a refundable ITC of $35 \%$ of qualifying current and capital SR\&ED expenditures, to a maximum of $\$ 3$ million of expenditures per year. Over the \$3 million SR\&ED expenditure limit, the credit rate is reduced to $20 \%$, but not refundable. The $\$ 3$ million expenditure limit is reduced to zero on a phased basis as the CCPC's taxable income rises from $\$ 500,000$ to $\$ 800,000$ and its taxable capital increases from $\$ 10$ million to $\$ 50$ million.

- Large CCPCs with prior-year taxable income of $\$ 800,000$ or more or with prior-year taxable capital employed in Canada of $\$ 50$ million or more or non-CCPCs (foreign controlled corporations) to claim an ITC of $20 \%$ of qualifying current and capital SR\&ED expenditures. The tax credit is not refundable.

The upper limit on taxable income was increased from $\$ 600,000$ in Budget 2008 and $\$ 700,000$ in Budget 2009. The upper limit for taxable capital was increased from $\$ 15$ million in Budget 2008. A summary of this tax credit can be found in Table 3.B-2.

\subsubsection{Costs of Indirect Measures of the Federal Government}

With respect to tax expenditures on $R \& D$, there is to date no official data or estimates by province of the costs of the federal government. Neither the Canadian federal Department of Finance, nor Statistics Canada, publishes these data. The only data available are aggregated expenditure published by the Department of Finance (see for example Department of Finance (2010b)).

The panel expert mandated by the federal government for all key programs of the federal portfolio of incentives to support business R\&D and R\&D for commercial purposes addressed in its final report (Industry Canada (2011), page 3-12) the issue of the lack of information on federal spending by province and found it difficult to ascertain the geographical distribution of tax credits for SR\&ED. Based on work 
covering the period 2000 to 2007, they found that between 40 and $50 \%$ the tax credit for SR\&ED was requested by companies belonging to several provinces, but have provided, as a rule, the data according to the head office location. Therefore, it was not possible to establish precisely which region these firms operate in SR\&ED, nor to draw general conclusions about the distribution of SR\&ED claims by region. Given this important caveat, the analysis of the expert group indicates that the average distribution of the estimated tax credit for SR\&ED for companies operating in only one province seems to closely correspond to the average provincial distribution of BERD, that is to say, especially in Ontario and Québec, to a lesser extent in the four western provinces, and some in the Atlantic provinces.

Table 3.4-1: Estimate of tax expenditure, total Canada, 2005-2010

\begin{tabular}{|c|c|c|c|c|c|c|}
\hline & \multicolumn{6}{|c|}{ (\$millions) } \\
\hline & \multicolumn{4}{|c|}{ Estimates } & \multicolumn{2}{|c|}{ Projections } \\
\hline & 2005 & 2006 & 2007 & 2008 & 2009 & 2010 \\
\hline \multicolumn{7}{|l|}{ Scientific Research and Experimental Development } \\
\hline Earned and claimed in current year & 2050 & 2135 & 2220 & 2420 & 2310 & 2450 \\
\hline Claimed in current year but earned in prior years & 580 & 590 & 1045 & 900 & 860 & 910 \\
\hline Earned in current year but carried back to prior years & 90 & 90 & 85 & 165 & 110 & 110 \\
\hline Total tax expenditure & 2720 & 2815 & 3350 & 3485 & 3280 & 3470 \\
\hline
\end{tabular}

Source: Tax Expenditures and Evaluations 2010, Canada, Department of Finance, Canada.

If we assume that this hypothesis is true, and knowing that the proportion of BERD for Québec and Ontario were respectively $29.1 \%$ and $47.9 \%$ of the Canadian total (BERD data in Table 3.E-14), one can quickly estimate that, for Québec and Ontario, the total amounts of tax credits for 2008 were approximately $\$ 1,014$ million (3 $485 \times 29.1$ ) and \$1,669 million (3 $485 \times 47.9$ ) respectively, all other things being equal. However, it is very rough estimates because the total tax credit to which they are calculated include companies operating in several provinces and those that operate in only one province.

\subsubsection{Direct Support Measures}

The Government of Canada provides direct funding for R\&D and innovation primarily through the NRC which is the Government of Canada's leading resource for R\&D and technology-based innovation. The main instrument available to the NRC to stimulate the innovative capacity of SME is the NRC Industrial Research Assistance Program (NRC-IRAP), a program implemented at least 60 years ago. Support from the program could cover up to $50 \%$ of R\&D costs of certain projects. Funding usually ranges between $\$ 5000$ and $\$ 350000$ depending on the complexity of the project. Besides financial support, NRC also offers technical support to SMEs at all stages of their process of innovation through its 20 institutes 
and national programs, spanning a wide variety of disciplines and offering a broad array of services.

Other federal R\&D incentives are offered by various governmental agencies such as the Industrial Technologies Office (ITO), an Agency of Industry Canada, which provides financial support for leading edge R\&D by Canadian industries. The agency delivers the Strategic Aerospace and Defence Initiative (SADI) and manages existing projects from the Technology Partnerships Canada (TPC) ${ }^{9}$ program and the Program for Strategic Industrial Projects (PSIP). SADI supports Canadian aerospace and defence industries, specifically by encouraging strategic $R \& D$ that will result in innovation in new products and services and foster collaboration between research institutes, universities, colleges and the private sector. ITO's financial support for this program may equal 30 percent of a project's total eligible costs.

Finally, another federal direct support program available to firms in Québec is the Canadian Space Agency (CSA) Class Grant and Contribution Program that provides financial support to organizations that conduct space related R\&D in CSA priority areas. Financial support for this program varies accordingly to project.

\subsubsection{Costs of Direct Measures of the Federal Government}

As shown in Figure 3.4-1, the subsidies to the sector of high education topped the list with more than $66 \%$ of the total amount spent in Québec in 2003-2004 by the federal government and its agencies, what is also similar to the treatment of direct support programs by the Government of Québec. However, the resemblance of treatment stops there. First, in absolute value, the total federal spending for subsidies to R\&D is much more important than those of the Government of Québec for the same year; they represent $\$ 777$ million compared to $\$ 492$ million (Table 3.C-7 and Table 3.C-6). Also, with regard to the other performers, the treatment of subsidies by the federal government is not the same as the Government of Québec: the federal payments to companies exceeded \$221 million in 2003-2004, giving a higher percentage than that of the Government of Québec, $28 \%$ against $7 \%$.

\footnotetext{
${ }^{9}$ Technology Partnerships Canada was a special operating agency of Industry Canada with a mandate to provide funding support for strategic R\&D, including environmental technologies, life sciences, information and communications technologies and advanced manufacturing. The terms and conditions of the Technology Partnerships Canada program expired on December 31, 2006.
} 
Figure 3.4-1: $\quad$ Federal expenditure on direct programs for R\&D, by sectors, 2003-2004

- Business enterprise Higher education ${ }^{-}$Other performers

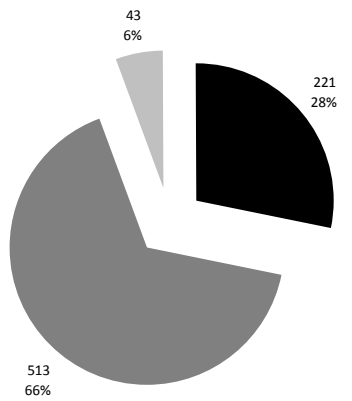

Source: Statistics Canada, Statistics Canada, Table 358-0150, CANSIM.

Figure 3.4-2: Evolution of Federal expenditure on direct programs for R\&D, by sectors, 2003-2004 to 2008-2009

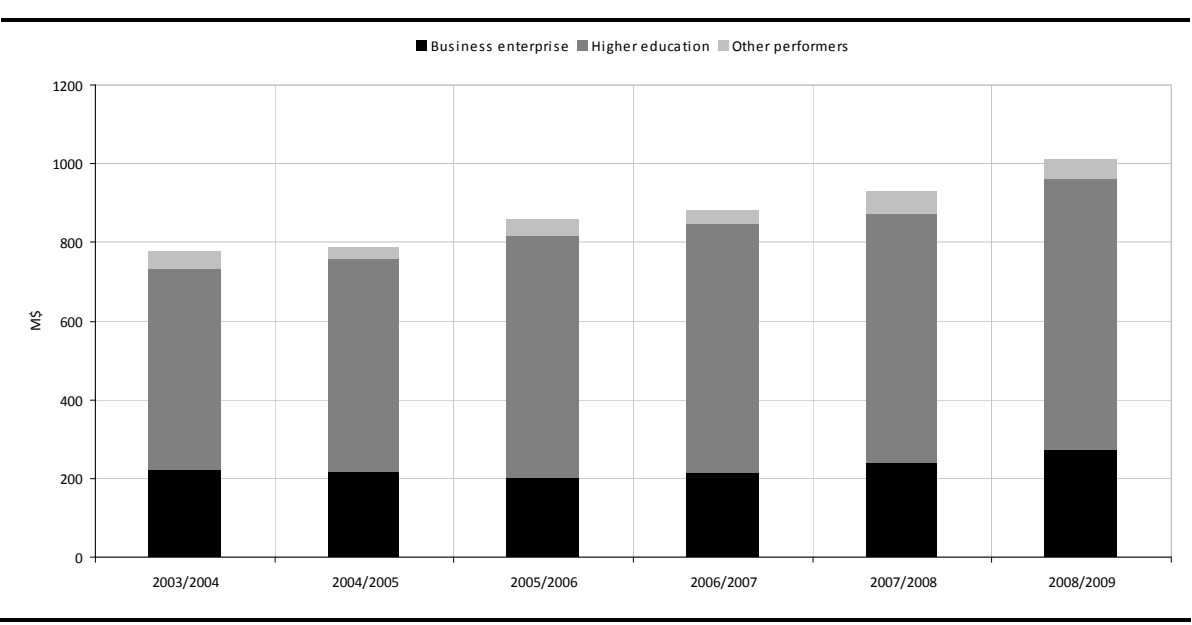

Source: Statistics Canada, Statistics Canada, Table 358-0150, CANSIM.

\subsection{Net Cost of the Combined Federal-Provincial Indirect Support Measures}

A firm located in Québec that incurred R\&D expenditures may claim the SR\&ED tax credit for salaries and wages of researchers at the provincial level and the SR\&ED tax credit at the federal level according to formulas and rates respectively in 
Section 3.3.1 (provincial) and Section 3.4.1 (federal). In Table 3.D-9 and Table 3.D-9 we present a few examples of the net cost of $\$ 100,000$ R\&D expenditures incurred respectively by SMEs and by large corporations in the manufacturing sector. Note that, the tax rates shown in these appendices apply to the year 2003. It appears, for example, that the net cost for an SME is $\$ 28,600$ and $\$ 58,400$ for a large firm. In other words, for every dollar spent, an SME may receive a fiscal subsidy of $\$ 72.4$ and a large firm receive \$41.6.

In order to make these net costs more meaningful, we compare them to those of Ontario, Canada as a whole and several countries around the world. As can be seen in Figure 3.5-1, in 2006-2007, in the case of large firms, only Spain provided a tax subsidy (c0.39) higher than that offered by Québec (c0.37). The Canadian average was less than twice the subsidy offered by Québec and the United States

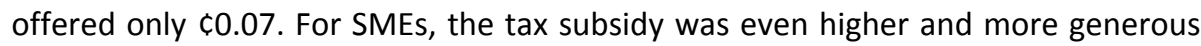
among all the countries examined: it was $\ 0.73$, followed by Ontario with $\$ 0.65$. The difference is significant between these two neighboring provinces and other countries generally recognized as offering generous tax subsidies such as Spain (c0.39), Mexico (c0.37) and China (c0.34). Thus, these figures confirm that Québec has one of the most generous tax regimes in the world.

Figure 3.5-1: Rate of fiscal subsidy for a dollar of R\&D, Québec, Ontario, Canada and selected countries, large firms, 2006-2007

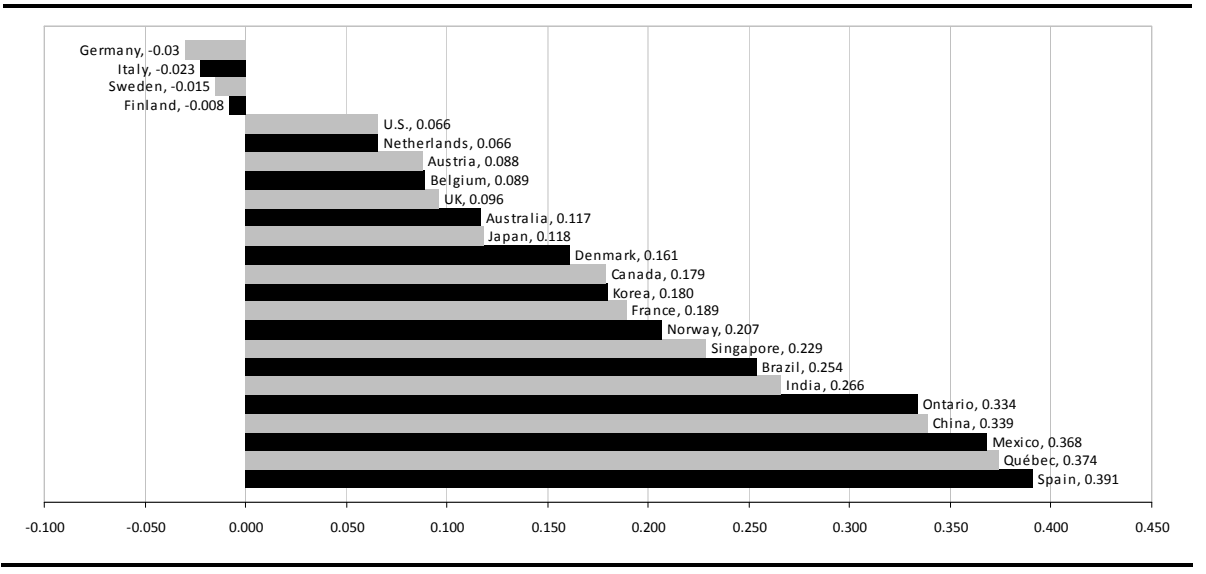

\section{Adapted from: $\quad$ CST (2010).}

Note: For Québec and Ontario, the data reflect the provincial and federal tax assistance while for Canada, only federal assistance is considered. For all countries, only the central government tax assistance is considered. To our knowledge, in almost all countries, only the central government provides tax assistance for R\&D. 
Figure 3.5-2: Rate of fiscal subsidy for a dollar of R\&D, Québec, Ontario, Canada and selected countries, SMEs, 2006-2007

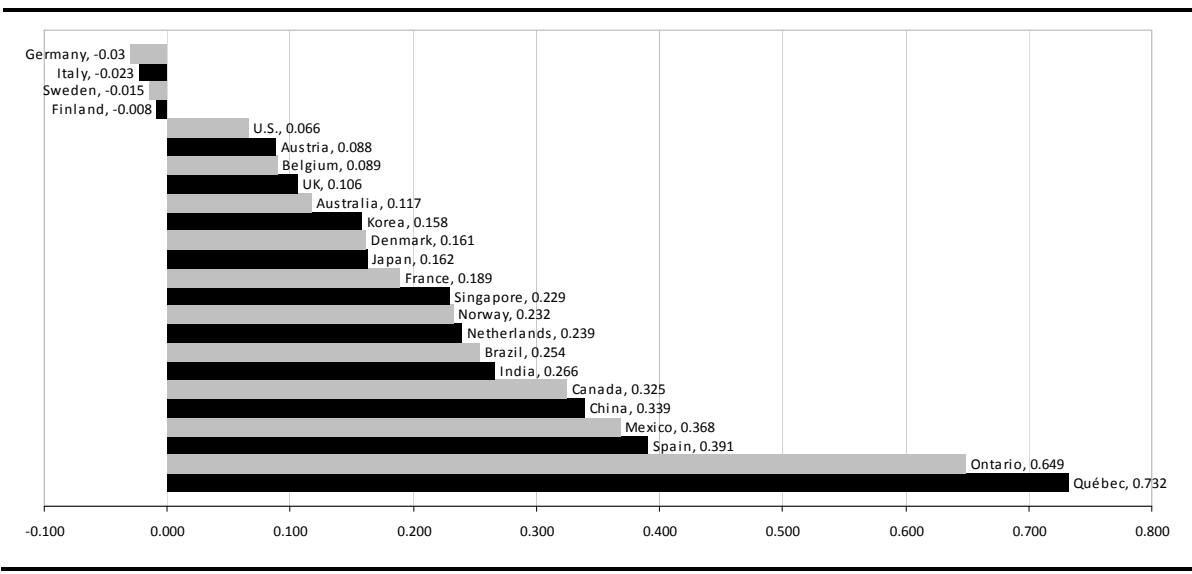

Adapted from: $\quad$ CST (2010)

Note: For Québec and Ontario, the data reflect the provincial and federal tax assistance while for Canada, only federal assistance is considered. For all countries, only the central government tax assistance is considered. To our knowledge, in almost all countries, only the central government provides tax assistance for R\&D.

\subsection{Interprovincial Comparison of the Main Tax Credits for R\&D}

The comparison of the tax systems of Canadian provinces and territories shows that the province of Québec stands out significantly by the extent and nature of the measures in place. This is partly due to historical reasons, as Québec was the first Canadian province to offer and to administer its own incentives for R\&D by introducing the SR\&ED tax credit in 1983. As can be seen in Table 3.B-4 that gives the summary of the various support measures for all Canadian provinces and territories as well as those of the Government of Canada in historical perspective, other Canadian provinces and territories have been added over time and, since 2000, the Territory of Yukon is the last Canadian jurisdiction to provide tax assistance for R\&D. To date, a province, Prince Edward Island and two territories, Nunavut and Northwest territories, do not offer tax incentives for R\&D. Most of the Canadian provinces that offer tax incentives made a tax collection agreement with the federal government entrusting the administration of their programs of R\&D tax credit to Canada Revenue Agency. Thus, provincial tax credit is claimed in conjunction with federal SR\&ED tax credits. With Québec, Alberta is the only other Canadian province that administers itself its tax system to encourage research and development. However, in Alberta as in other Canadian provinces, except Québec, 
the nature of tax credit usually mirrors that granted by the federal government. In these provinces, tax support for corporations is generally based on current expenditures and capital eligible with R\&D credit rate up to $20 \%$ so far. Meanwhile, in Québec, the tax credits are primarily based on expenditures related to salaries of researchers. Québec is the only Canadian province that specifically targets human capital with its R\&D wage tax credit whose rates reach $40 \%$.

\subsection{Statistics on R\&D and Innovation in Québec}

\subsubsection{R\&D Intensity}

By examining the evolution over the last thirty years of the main statistical measure for the level of investment in Québec, that is, the Gross domestic expenditure on $R \& D$, abbreviated as GERD, which includes expenditure on research and development by business enterprises, Higher education institutions, as well as government and private non-profit organizations, we see that it has more than quadrupled, passing from $\$ 1,465$ million in 1981 to $\$ 6,503$ million in 2008 , in constant dollars of 2002 (Table 3.E-11). This represents an average annual growth rate of $5.68 \%$.

However, a measure that gives a better picture of the investment effort is the ratio of GERD to GDP, also known as the intensity of R\&D. It has also become the standard for target levels of investment in industrialized countries.

In Figure 3.7-1 that shows the evolution of the intensity of R\&D one can see that in the early 80's, Québec ranked in third position of the Canadian provinces, after Ontario and Nova Scotia and was lagging behind Canada as a Whole. This trend was reversed during the 90s, making Québec the first province in terms of intensity of R\&D since 1998. In 2008, this ratio was $2.6 \%$ after reaching a Canadian peak of $2.8 \%$ between 2001 and 2004 and in 2006. Ontario with 2.4\% in 2008 ranks second and has maintained this position since 1998. Moreover, among Canadian provinces, Ontario is the only Canadian province with Québec to remain for years above the national average which stood at 1.9\% in 2008 (Figure 3.7-2).

However, despite this strong growth in Québec's R\&D intensity, when looking at the evolution of the intensity of $R \& D$ over the period 1981 to 2008 in Figure 3.7-1, it appears clearly that since the 2001 break in the trend of the curve of the investments in R\&D in Québec that followed the peak of $2.8 \%$ mentioned above, R\&D intensity seems to stagnate. This decline in trend is also reflected in the curve of the investments in R\&D in Canada and in Ontario. The evidence suggests that this stagnation is the result of the economic effects arising from September 11 attacks. 
Figure 3.7-1: Evolution of R\&D intensity, Québec and Canadian provinces, 1981-2008

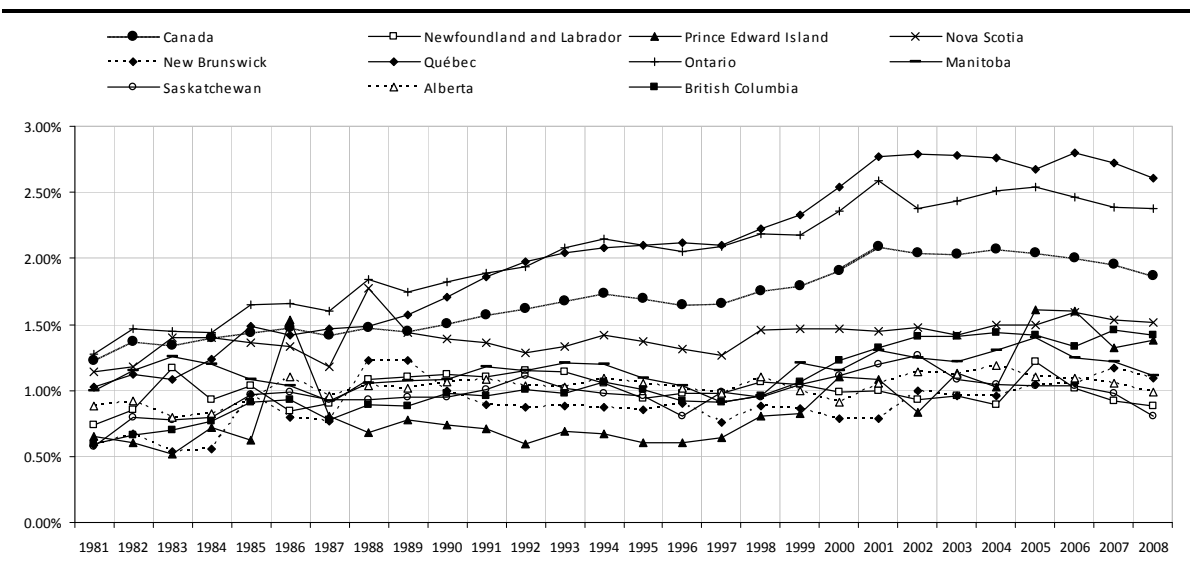

Source: Statistics Canada, Table 358-0001 - Gross domestic expenditure on research and development, Table 384-0002 - Gross domestic product (GDP), CANSIM (database).

The break in the trend is not without consequences in the government policy. Indeed, one of the main policy objectives of R\&D and innovation conducted in Québec in recent decades by both the Canadian and the Government of Québec is to promote the overall level of investment in R\&D which is considered essential to productivity growth and to the competitiveness of the economy as a whole. Thus, like the OECD countries, Québec has also set its own targets for R\&D intensity. The first goal was set in the Action Plan 1988-1992 (Gouvernement du Québec (1988)), the government's goal was to raise total R\&D spending to $2 \%$ of GDP in 1992 . In 2001, the Government of Québec published a new policy of science and innovation which aimed at inducing increased investment effort so that the percentage of R\&D spending relative to GDP, which stood at $2.09 \%$ in 1998 , joins the average of the G-7, set to $2.47 \%$ for that year, by the year 2006-2010 (Gouvernement du Québec (2001), pp. 37). In 2004, in a new policy statement, the Government of Québec announced the objective to increase the share of R\&D spending to $3 \%$ of GDP by the end of the mandate, i.e. in 2007 (Gouvernement du Québec (2004)). In 2005, in a statement on its economic development strategy (Ministère du Développement économique (2005)), the government made an update to its goal. Thus, the target of $3 \%$ of GDP in research expenditure is pushed back to 2010. At this rate, the government plans were to increase R\&D spending to nearly $\$ 10$ billion in 2010, representing an average annual growth of $5.2 \%$, and also aims to raise the share of firms in financing the R\&D of $60 \%$ in 2002 to over $66 \%$ in 2010, that is, from $\$ 3.9$ billion to $\$ 6.6$ billion. The examination of the ratio GERD/GDP, in current prices since it reflects changes in price as well as real changes in the level of expenditure, shows that the overall target of $3 \%$ were not reached in 2008 (Figure 3.7-1). 
In an update of its economic development strategy, published in 2010 (Ministère du Développement économique (2010)), the government announced no new target. It merely states that the government maintains the target it set for itself, i.e. to reach $3 \%$ of GDP in R\&D expenditures.

Figure 3.7-2: R\&D intensity, Québec and Canadian provinces, 2008

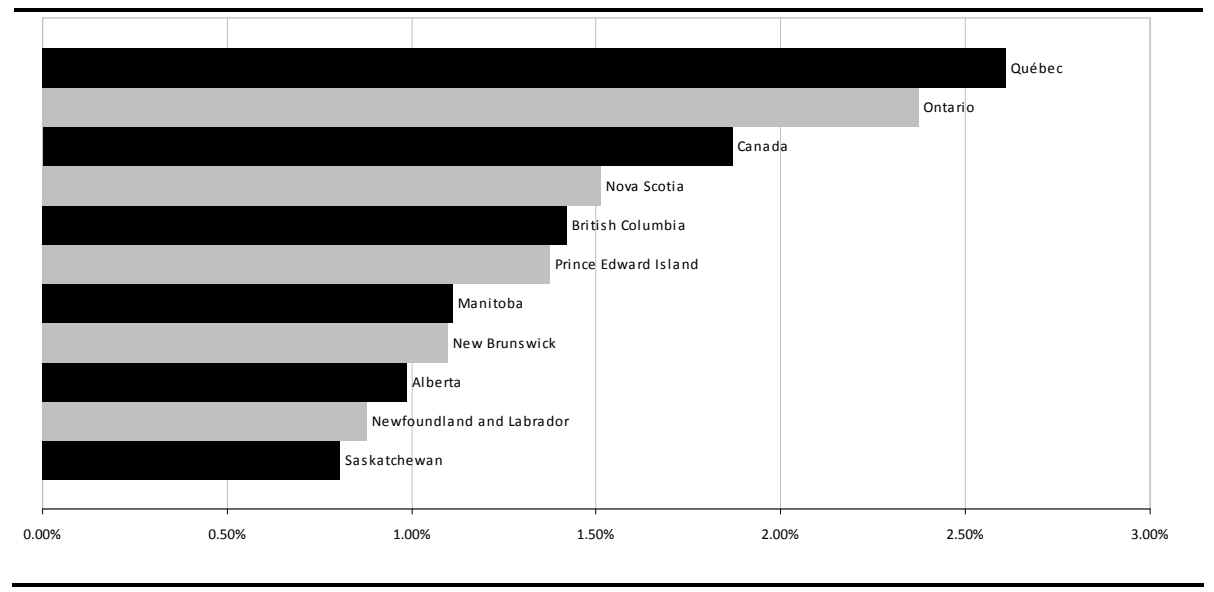

Source: Statistics Canada, Table 358-0001 - Gross domestic expenditure on research and development, Table 384-0002 - Gross domestic product (GDP), CANSIM (database).

Dividing the Gross domestic expenditure on R\&D by performer provides an overview of the source of funds contributing to R\&D intensity. Thus, Figure 3.7-3 shows the evolution of real GERD in Québec from 1981 to 2008 along with the contribution of each R\&D sector. As it appears, there are six main R\&D investors in Québec which are in order of importance, the business enterprise sector, the higher education sector, the federal government and its agencies, the Government of Québec and its research organizations and private non profit organizations. Over the period between 1981 and 2008, the first two, i.e the business enterprise sector and the higher education sector, have contributed annually to over $88 \%$ of total R\&D in Québec. Investment in R\&D of business sector, which exceeded $58.2 \%$ in 2008 , has grown by an average of $5.63 \%$ over the same period. On the other hand, the sector of higher education, the second most important investor with $35.4 \%$ of the total investment, recorded a faster growth than the sector of business enterprise over this period, i.e. $6.42 \%$ against $5.63 \%$. The reason resides in the payment in recent years to the sector of higher education of increasingly significant subsidies, notably by the Government of Québec, to encourage commercially oriented university research through cooperation programs between universities; provincial research organizations and industry. 
The other R\&D investors share the remaining 7\%, in particular the federal government and its agencies (5.3\%), the Government of Québec and its research organizations (1.2\%) and the rest by non-profit organizations.

Figure 3.7-3: Evolution of real GERD, by sector, Québec, 1981-2008

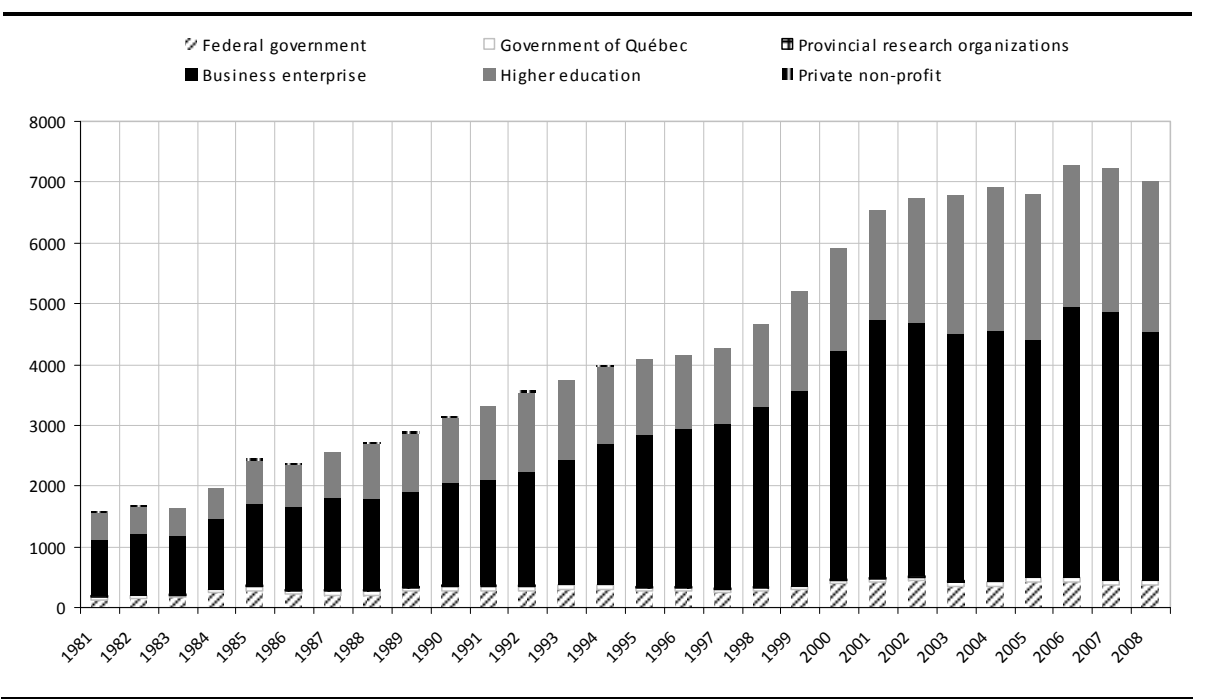

Source: Statistics Canada. Table 358-0001 - Gross domestic expenditure on research and development, Table 384-0036 - Implicit price indexes, gross domestic product (GDP), provincial economic accounts, annual (index, 2002=100), CANSIM (database).

The comparison of sources of funds for investment in R\&D in Québec with those of Ontario and Canada shows a similar distribution in general (Figure 3.7-4). However, we note that the investments of the Government of Canada in percentage of GDP are higher in Ontario than in Québec.

Turning to our area of interest, the business enterprise sector, we first examine the evolution of R\&D investments in this sector. As shown in Figure 3.7-5, the curve of the investments in R\&D of business enterprise mirrors that of the total investments in $R \& D$, first passing from $0.6 \%$ in 1981 to $1.8 \%$ in 2001 with sustained growth and then remained stagnant after this period with rates ranging between $1.5 \%$ and $1.7 \%$. This confirms that Québec businesses were also affected by the financial crisis that followed the events of September 11, 2001. 
Figure 3.7-4: Comparison of R\&D intensity, by performer, Canada, Québec and Ontario, 2008

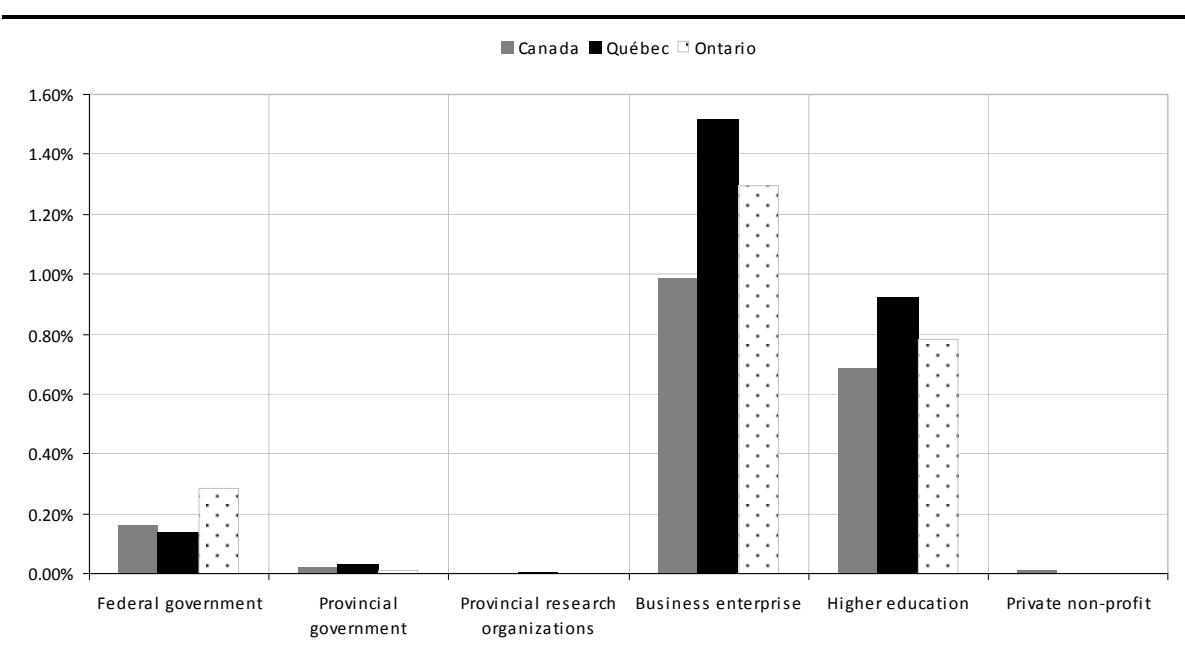

Source: Statistics Canada, Table 358-0001 - Gross domestic expenditure on research and development, Table 384-0002 - Gross domestic product (GDP), CANSIM (database).

Figure 3.7-5: Evolution of R\&D intensity, business sector, Québec and Canadian provinces, 1981-2008

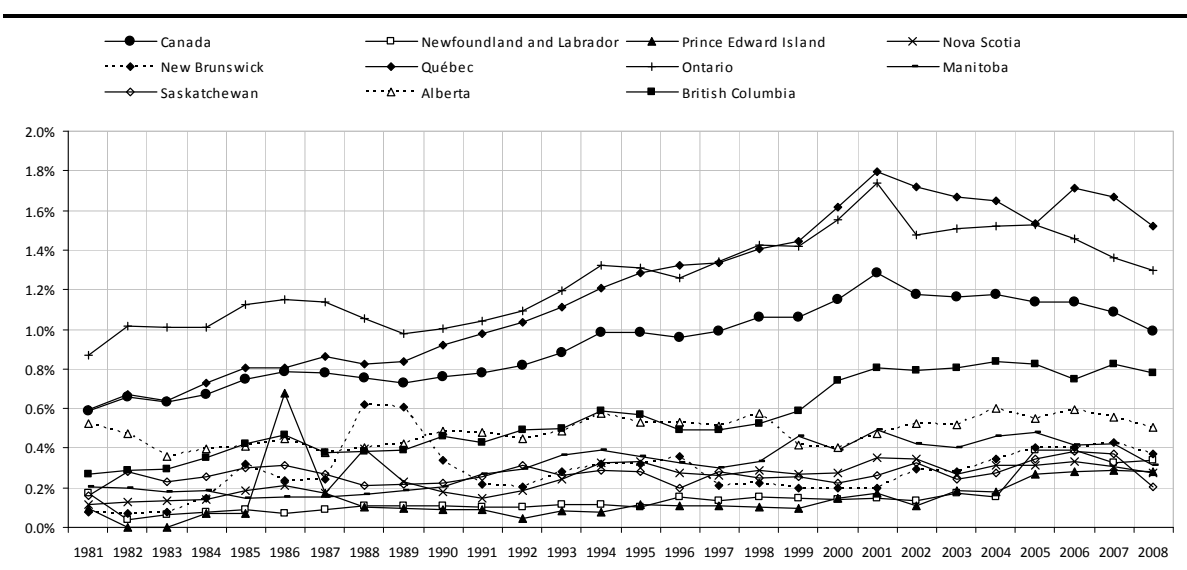

Source: Statistics Canada, Table 358-0001 - Gross domestic expenditure on research and development, Table 384-0002 - Gross domestic product (GDP), CANSIM (database).

To put the above indicators in perspective to the global scale, we compare them to those of the largest investors in R\&D worldwide. Figure 3.7-6 shows the comparative evolution of Gross domestic expenditure on R\&D in percentage of GDP in Québec, Canada, Finland, Japan, USA and OECD between 1997 and 2003. GERD of Québec passed from $2.13 \%$ to $2.78 \%$ of its gross domestic product in 2003. In terms 
of R\&D expenditures, this represents about 5.5 billion dollars in PPP. For the same year, only four OECD countries showed a ratio higher than that of Québec, namely Sweden, Finland, Japan and Iceland (see Table 3.E-13 for full data of Canadian provinces and OECD countries, 1997-2003).

Figure 3.7-6: Evolution of R\&D intensity, Québec, Canada and selected OECD countries, 1997-2008

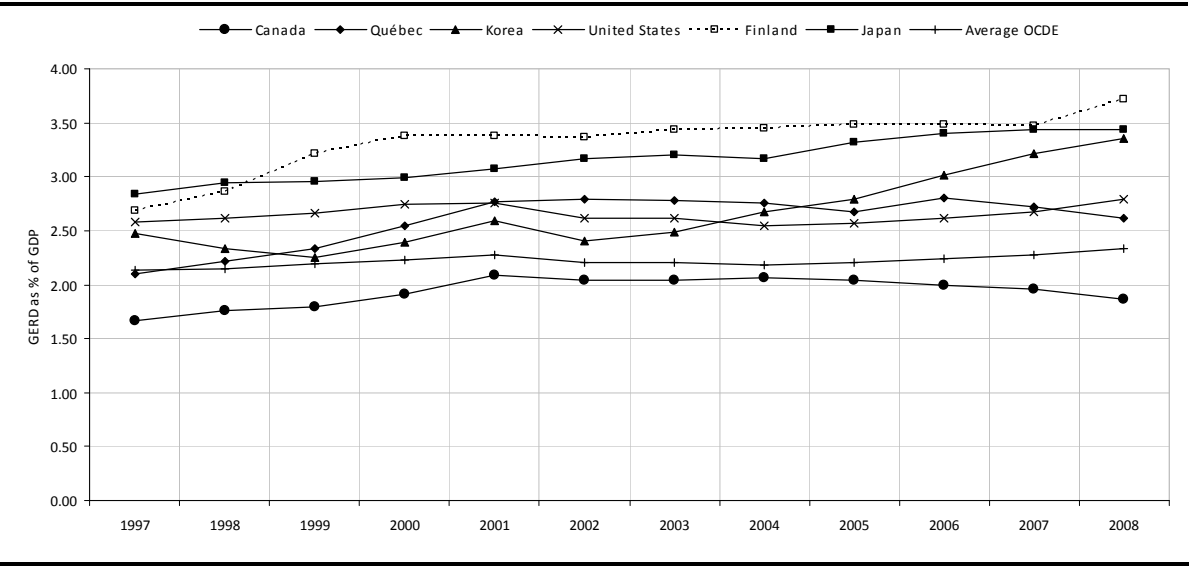

Sources: Institut de la statistique du Québec, Indicateurs de l'activité scientifique et technologique au Québec, Compendium, Éditions 2006 et 2011. For Québec and Canada data, Statistics Canada., Table 358-0001, CANSIM.

In the business sector, R\&D spending as a percentage of Québec's GDP grew by $1.36 \%$ to $1.66 \%$ between 1997 and 2003 with a peak of $1.82 \%$ in 2001 . This is reflected significantly in R\&D spending in Canada which, as shown in Figure 3.7-7, followed the same trend as in Québec. Compared to OECD countries, R\&D spending as a percentage of Québec's GDP in the business sector has placed itself in seventh position behind Sweden, Finland, Japan, Korea, United States and Denmark (see Table 3.E-14 for full data of Canadian provinces and OECD countries in 1991 and 1997-2003). 
Figure 3.7-7: Evolution of R\&D intensity, business sector, Québec, Canada and selected OECD countries, 1997-2008

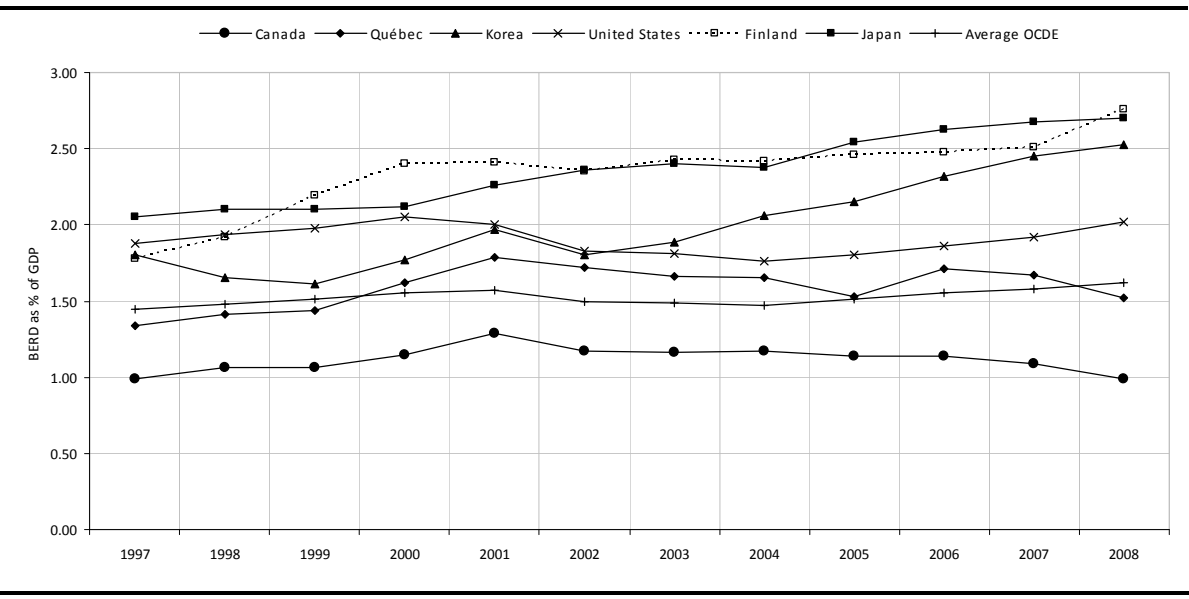

Sources: Institut de la statistique du Québec, Indicateurs de l'activité scientifique et technologique au Québec, Compendium, Éditions 2006 et 2011. For Québec and Canada data, Statistics Canada. Table 358-0001, CANSIM.

\subsubsection{R\&D Personnel}

The number of employees assigned to $R \& D$ activities, or simply $R \& D$ personnel, is composed of persons employed directly within R\&D, as well as those supplying direct services to R\&D, such as managers, administrative staff and clerical staff. These do not include persons providing an indirect service, such as canteen and security staff. Among the personnel engaged in R\&D, are the researchers whose statistics are closely monitored. Researchers include professionals engaged in the conception or creation of new knowledge, products, processes, methods and systems, and in the management of the projects concerned. Given the fact that supply and demand of human resources devoted to R\&D and innovation are critical to the development of knowledge and competitiveness of an economy, R\&D personnel is a key indicator for measuring the knowledge-based economy and its developments. Furthermore, in the case of Québec, given the fact that the fiscal programs supporting R\&D are heavily weighted towards subsidizing R\&D staff, this statistic is of particular importance and may be considered as an outcome measure for the effectiveness of these programs.

The portrait of the recent situation with regard to R\&D staff is as follows in Québec. Over the period 2002-2008, the number of employees $\left(\mathrm{FTE}^{10}\right)$ assigned to

\footnotetext{
${ }^{10} \mathrm{FTE}$, acronym for full-time equivalent, may be thought as a unit to measure employed persons in a way that makes them comparable although they may work a different number of hours per week. The unit is obtained by comparing an employee's average number of hours worked to the average number of hours
} 
R\&D in Québec has increased, passing from 57040 to 75180 (see Table 3.E-16). This represents an average annual growth rate of $4.7 \%$. In comparison, the growth rate is slightly lower than that of Canada as a whole (4.8\%) and higher than that of Ontario (4.3\%) for the same period. However, one should note in the case of Ontario that the lag in R\&D personnel is not attributable to a trend, but a significant decline between 2007 and 2008.

The examination of the ratio of the number of researchers on the total number of workers in R\&D over the period 2002-2008 in Table 3.E-16 also reveals that the proportion of researchers in Québec remained lower than those of Ontario and Canada as a whole. Thus, in 2008, the share of researchers in total employees engaged in R\&D in Québec stood at $57.6 \%$ while it stood at $63.4 \%$ in Ontario and $61.4 \%$ for Canada. In addition, over the same period, this ratio has declined slightly from $60.7 \%$ and to $57.6 \%$. But this last statement is also true in the cases of Canada and Ontario.

Figure 3.7-8: Proportion of R\&D personnel, by Sector, Québec, Canada and selected provinces, 2005

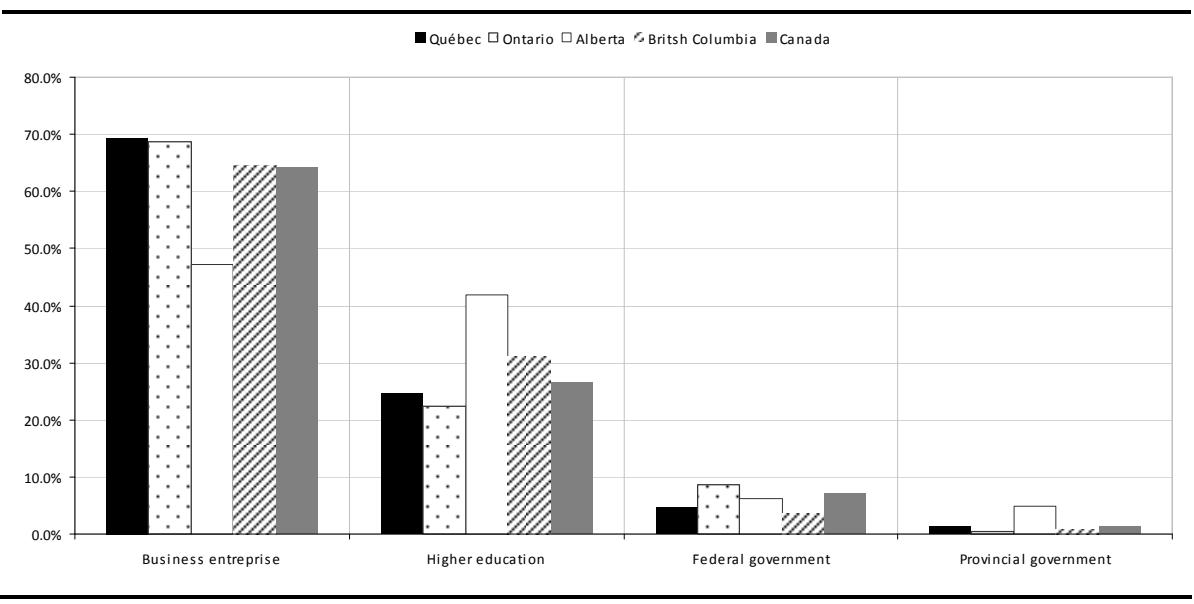

Source: Statistics Canada, no 88-001-X, May 2008, compiled by the author.

A breakdown of R\&D personnel by sector in 2005 (Figure 3.7-8, full data in Table 3.E-17) shows that nearly $70 \%$ of the total were concentrated in the business enterprise sector and the higher education sector (25\%). The federal and provincial government sectors share the remaining, $4 \%$ and $1 \%$ respectively. The pattern was quite similar in Canada, Ontario and British Columbia. In Alberta the ratio of the corporate sector and higher education were more evenly distributed. As can be seen, with a ratio of nearly $70 \%$, the province of Québec ranked first among

of a full-time worker. Hence, a full-time R\&D worker who spends $30 \%$ of his time on R\&D and the rest on other activities should is counted as 0.3 FTE. 
Canadian provinces in term of R\&D personnel in the business sector. However, the gap with other top R\&D performers in Canada was not important. Indeed, Ontario and British Columbia followed closely with ratios around $69 \%$ and $65 \%$ respectively.

Figure 3.7-9: Ratio of researchers to R\&D personnel, all sectors, Canada and provinces, 2005

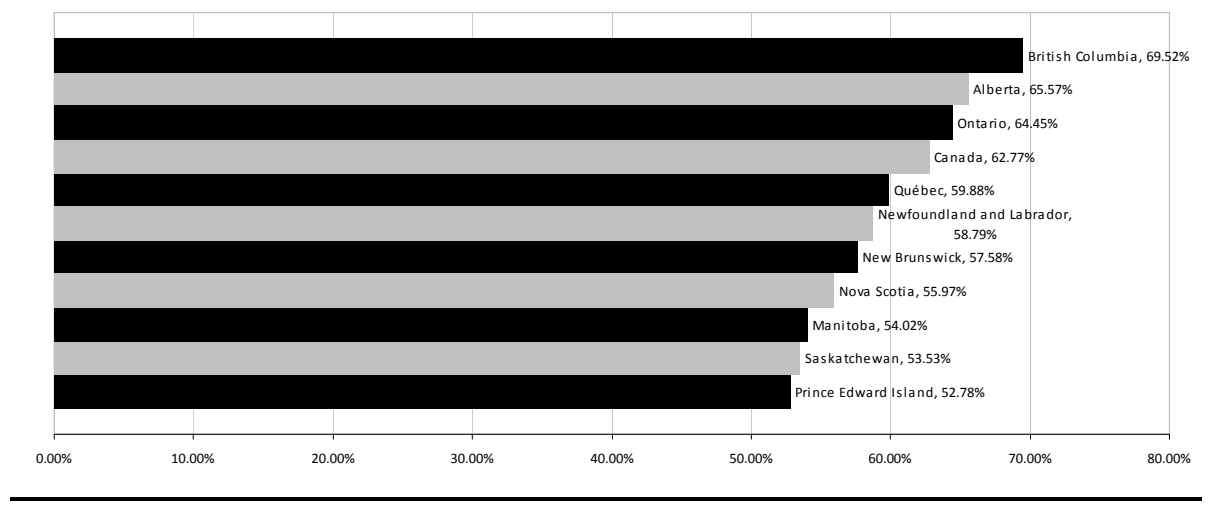

Figure 3.7-10: Ratio of researchers to R\&D personnel, business sector, Canada and provinces, 2005

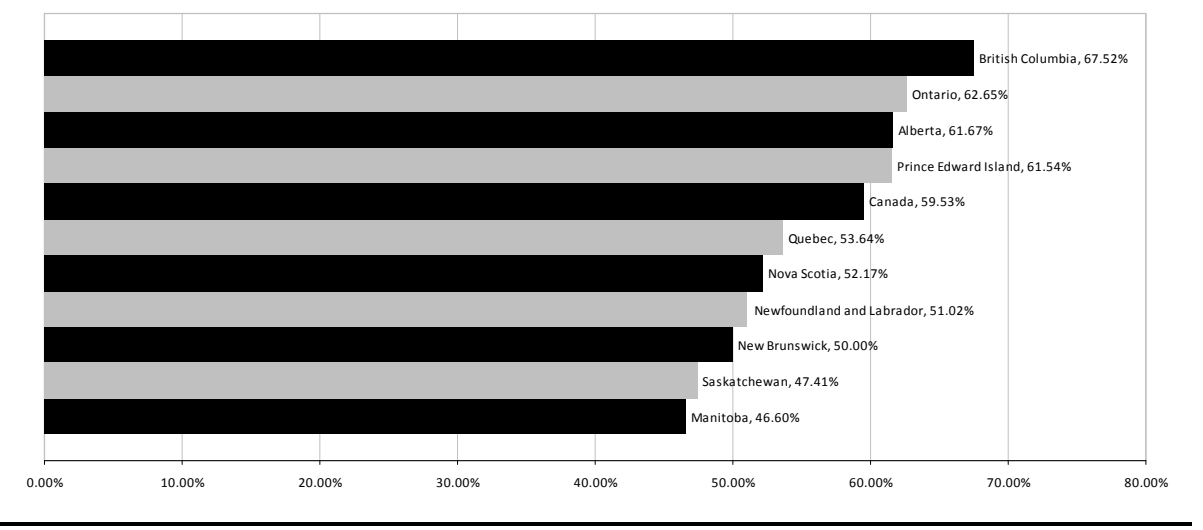

Source: Statistics Canada, no 88-001-X, May 2008, compiled by the author.

Finally, by comparing the ratio of the number of researchers to R\&D personnel for all sectors (Figure 3.7-9) and in the business sector (Figure 3.7-10) in 2005, one can see that in both cases Québec ranked below the Canadian average.

In the light of the statistics presented above, one conclusion that emerges is that, since the early 1980s, despite the fact that Québec businesses receive the most advantageous tax assistance program in Canada for jobs related to R\&D, the 
proportion of researchers has, so far, failed to increase significantly compared to the national average. Indeed, as shown in the data, this proportion has continued to lag behind Ontario and Canada as a whole in recent years.

\subsection{R\&D Intensity and the Québec's Productivity gap Issue}

Labour productivity growth is a key indicator to measure the prosperity of an economy. It positively affects the GDP and thus, the standard of living. Most economists agree that the intensity of R\&D is a prime contributor to its growth. Numerous studies have shown a positive link between investment in R\&D and labour productivity growth (Wakelin (2001), Smith, Dilling-Hansen, Eriksson and Madsen (2004), Maté-Garcia and Rodriguez-Fernandez (2008)). The business sector is considered as the one that plays the leading role. We have shown in the previous section that R\&D investments of companies in Québec experienced a strong growth over the last thirty years and this has enabled them to reach and surpass the Canadian average. Today, Québec leads the Canadian provinces and OECD countries. This progress is often attributed to fiscal policy in place. A policy statement of the Government of Québec on science and innovation published in 2001 stated that this is attributed to tax credits for nearly two thirds of the catching up made by Québec since about twenty years (Gouvernement du Québec (2001), page 114). However, despite the convergence of the R\&D investments of Québec enterprises with those of the main countries investors in R\&D, the overall productivity of Québec continues to lag behind the latter and even behind its immediate neighbor, Ontario, whose growth rate of firms R\&D investments was less important during the same period. In a recent study, the HEC Montréal Centre for Productivity and Prosperity (CPP (2010)) emphasizes that Québec still lags far behind most OECD economies in terms of its standard of living, and over the period from 1981 to 2008, the standard of living gap between Québec and its immediate neighbours widened. Then, it shows that labour productivity has almost always been the main source of the standard of living gap between Québec and the rest of Canada and the United States before to conclude that it is attributable to the fact that Québec's industrial structure is more oriented toward lower-productivity sectors. For example, Québec's goods production sector has contributed very little to overall labour productivity growth since the mid-1980s and most of the labour productivity growth occurred in the service sector where the contribution to labour productivity has been historically low compared to Ontario's or Canada's service sector.

This finding raises the question of knowing whether this is due to poor performance of companies in this sector in Québec compared to those of other Canadian provinces, or merely to the small size of the goods production sector 
compared to the service and non-commercial sectors. Our analysis in Chapter 8 attempts to answer to this question, at least partially: from an econometric analysis, we compare the labour productivity of innovative manufacturing companies in Québec to that of innovative manufacturing companies from Canada as a whole and from OECD countries. 


\section{Chapter Appendices}

\section{A. Science and Technology Policies in Québec: Key Highlights, 1960-2010}

\begin{tabular}{|c|c|}
\hline Decade & Government intervention \\
\hline \multirow[t]{6}{*}{1960} & $\begin{array}{l}\text { - Creation of the Medical Research Council of Québec, ancestor of the Health Research } \\
\text { Fund of Québec (FRSQ). }\end{array}$ \\
\hline & o Creation of the Science Council of Canada, 1966. \\
\hline & - Creation of the Research Institute in electricity (IREQ), Hydro-Québec, 1967. \\
\hline & - Creation of the Industrial Research Centre of Québec (CRIQ), 1969. \\
\hline & - Creation of the Institute of Scientific Research (INRS), 1969. \\
\hline & $\begin{array}{l}\text { Creation of the Medical Research Council of Canada (MRC), ancestor of the Canadian } \\
\text { Institutes of Health Research, } 1969 .\end{array}$ \\
\hline \multirow[t]{7}{*}{1970} & $\begin{array}{l}\text { - Formation of a ministerial committee of science policy in Québec and publication of a } \\
\text { Statement of orientations. }\end{array}$ \\
\hline & $\begin{array}{l}\text { - Creation of the Training of researchers and concerted action (FCAC), ancestor of the } \\
\text { Fund for Training of researchers and research support (Fonds FCAR), } 1970 .\end{array}$ \\
\hline & $\begin{array}{l}\text { - Creation of the Conseil de la Politique Scientifique (CPS), the ancestor of the Conseil } \\
\text { de la Science et de la Technologie (CST), } 1971 .\end{array}$ \\
\hline & - Creation of the Social Sciences and Humanities Research Council (SSHRC), 1977. \\
\hline & $\begin{array}{l}\text { Creation of the Natural Sciences and Engineering Research Council of Canada } \\
\text { (NSERC), } 1978 .\end{array}$ \\
\hline & $\begin{array}{l}\text { - Publication of the green book Pour une politique québécoise de la recherche } \\
\text { scientifique, } 1979 .\end{array}$ \\
\hline & - Creation of the Québec Council of Social Research (CQRS), 1979. \\
\hline \multirow[t]{12}{*}{1980} & $\begin{array}{l}\text { - Adoption of the government policy of science and technology development Un projet } \\
\text { collectif, } 1980 .\end{array}$ \\
\hline & $\begin{array}{l}\text { - Publication of the economic agenda Technological change. Building Québec - Phase } 2 . \\
\text { Economic Action Program 1982-1986, } 1982 .\end{array}$ \\
\hline & $\begin{array}{l}\text { Elimination of the additional tax deduction of } 50 \text { per cent of increases in a taxpayer's } \\
\text { R\&D expenditures, } 1983 \text {. }\end{array}$ \\
\hline & - Implementation of the refundable tax credits of 10 per cent for salaries of R\&D, 1983. \\
\hline & $\begin{array}{l}\text { - Adoption of the Act to promote scientific and technological development, } \\
\text { transforming the CPS in the CST and creating the Fonds FCAR and the FRSQ, } 1983 .\end{array}$ \\
\hline & $\begin{array}{l}\text { - Establishment of centers of connections and transfer (CLT) and college technology } \\
\text { transfer centers (CCTT), } 1983 .\end{array}$ \\
\hline & - Creation of the National Advisory Board on Science and Technology (NABST), 1987 \\
\hline & ○ National Conference on Technology and Innovative, 1988. \\
\hline & o Creation of the Networks of Centres of Excellence (NCE), 1989. \\
\hline & $\begin{array}{l}\text { - Establishment of the laboratory of electrochemical technologies and } \\
\text { electrotechnology (LTÉÉ). }\end{array}$ \\
\hline & - Publication of Government strategy to support research and business development: \\
\hline & - Creation of the Technology Development Fund (FDT). \\
\hline
\end{tabular}




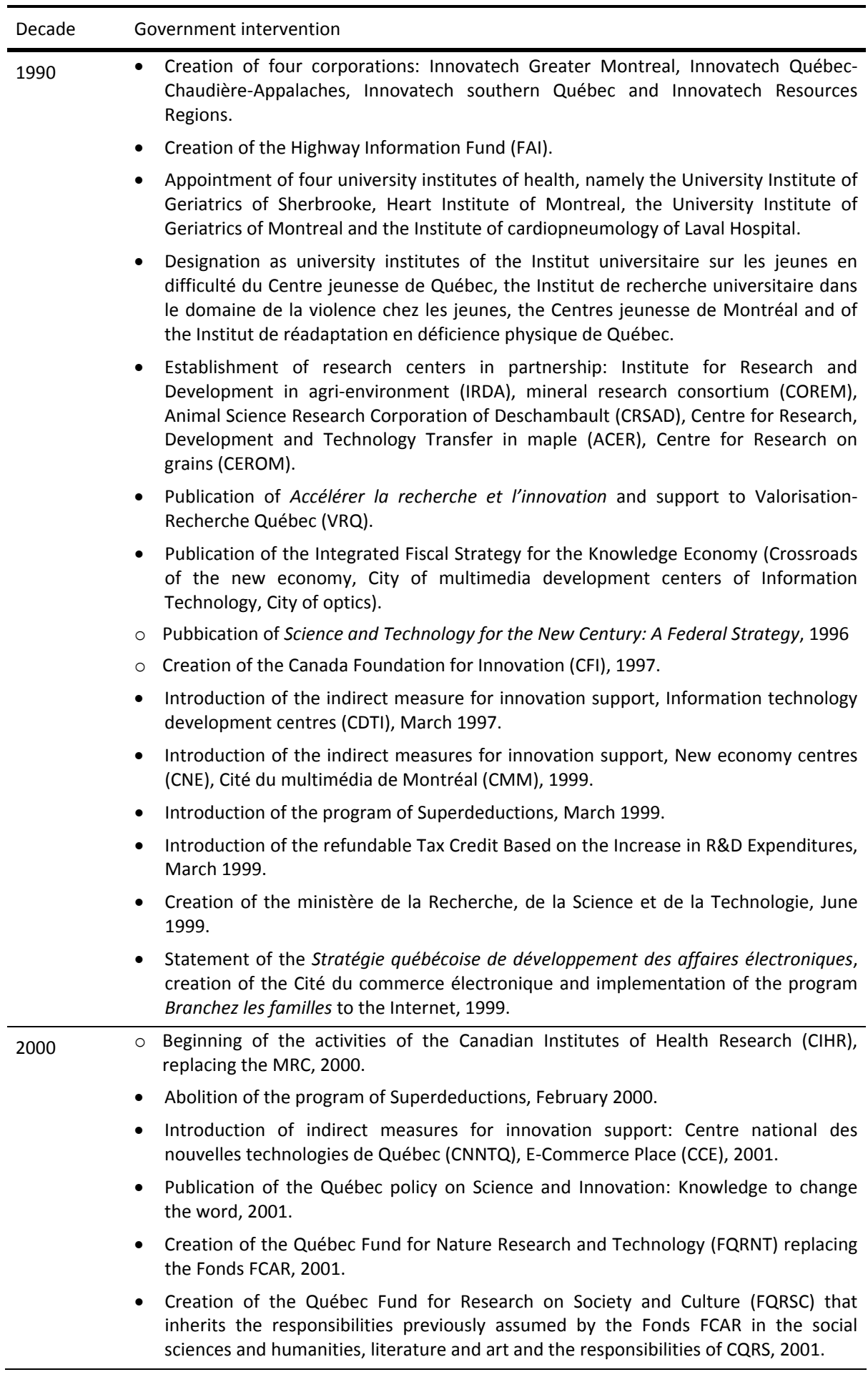




\section{Decade Government intervention}

- Introduction of the indirect innovation measure of the Montréal and Québec Innovation Centres, March 2002.

- Abolition of indirect measures for innovation support: CDTI, CNNTQ, CNE, CMM, CCE, Innovation Centres, 2003.

- Abolition of the refundable Tax Credit Based on the Increase in R\&D Expenditures, 2004.

- Publication of the L'Avantage québécois, 2005.

- Publication of the Stratégie québécoise de la recherche et de l'innovation: Un Québec innovant et prospère, 2006.

- Publication of the SQRI, Stratégie québécoise de la recherche et de l'innovation 20102013 : mobiliser innover prospérer, 2010.

Legend: • Events in Québec

- Events in Canada

Sources: Gouvernement du Québec (1988), CST (2002), Gouvernement du Québec (2001), Ministère du Développement économique (2005), Ministère du Développement économique (2006), Ministère du Développement économique (2010), author's compilation.

Note: The abbreviations used in this table are listed in page 265. 


\section{B. Summary of the Provincial and Federal Tax Credits Schemes}

Table 3.B-1: $\quad$ Summary of the Québec's SR\&ED tax credit rates, 2010 rates

\begin{tabular}{|c|c|c|c|}
\hline & Tax credit base & Tax credit rate & $\begin{array}{l}\text { Rate of } \\
\text { refundability }\end{array}$ \\
\hline \multicolumn{4}{|l|}{ Credit for salaries and wages* } \\
\hline $\begin{array}{l}\text { R\&D work carried out within } \\
\text { corporation }\end{array}$ & $\begin{array}{l}100 \% \text { of salaries and wages or } \\
100 \% \text { of sub-contracted work }\end{array}$ & $\begin{array}{l}37.5 \% \text { for } \text { SMEs }^{a} \\
17.5 \% \text { for large } \\
\text { firms }^{b}\end{array}$ & $100 \%$ \\
\hline \multicolumn{4}{|l|}{ Credits for R\&D expenditures } \\
\hline $\begin{array}{l}\text { R\&D work contracted out to: } \\
\text { University entity } \\
\text { Public research centre } \\
\text { Research consortium }\end{array}$ & $\begin{array}{l}80 \% \text { of the value of sub- } \\
\text { contracted work }\end{array}$ & $\begin{array}{l}35 \% \text { (effective } \\
\text { rate of } 28 \% \text { ) }\end{array}$ & $100 \%$ \\
\hline $\begin{array}{l}\text { R\&D work carried out by two } \\
\text { or more corporations : } \\
\text { Private partnership pre- } \\
\text { competitive research project }\end{array}$ & $100 \%$ of work done & $35 \%$ & $100 \%$ \\
\hline $\begin{array}{l}\mathrm{R} \& \mathrm{D} \text { work carried out by a } \\
\text { research consortium }\end{array}$ & $\begin{array}{l}100 \% \text { of membership fees or } \\
\text { dues paid }\end{array}$ & $35 \%$ & $100 \%$ \\
\hline
\end{tabular}

Source: Revenu Québec, Revenu Québec (2008), compiled by the author.

\section{Notes:}

${ }^{a}$ The $37.5 \%$ tax credit rate applies on the first $\$ 2$ million of annual salaries for corporations with assets under $\$ 50$ million. The rate applicable to qualified expenditures that exceed the first $\$ 2$ million is $17.5 \%$, regardless of the date of the expenditures or the amount of the corporation's assets. A linear reduction in the rate of the tax credit (from $37.5 \%$ to 17.5 ) applies to corporations with assets between $\$ 50$ and $\$ 75$ million.

${ }^{\mathrm{b}}$ The $17.5 \%$ tax credit rate applies to corporations with assets of $\$ 75$ million or more. 
Table 3.B-2: $\quad$ Summary of the Federal SR\&ED tax credit rates, 2010 rates

\begin{tabular}{|c|c|c|c|}
\hline \multirow[b]{2}{*}{ Business Type } & \multirow[b]{2}{*}{$\begin{array}{l}\text { Credit } \\
\text { Rates }\end{array}$} & \multicolumn{2}{|c|}{ Refundability Rates } \\
\hline & & $\begin{array}{l}\text { Current } \\
\text { Expenditures }\end{array}$ & $\begin{array}{l}\text { Capital } \\
\text { Expenditures }\end{array}$ \\
\hline Unincorporated businesses & $20 \%$ & $40 \%$ & $40 \%$ \\
\hline \multicolumn{4}{|c|}{$\begin{array}{l}\text { CCPCs with prior-year taxable income of } \$ 500,000 \text { or less and } \\
\text { prior-year taxable capital employed in Canada of } \$ 10 \text { million } \\
\text { or less: }\end{array}$} \\
\hline Expenditures up to expenditure limit ${ }^{a}$ & $35 \%$ & $100 \%$ & $40 \%$ \\
\hline Expenditures over expenditure limit & $20 \%$ & $40 \%$ & $40 \%$ \\
\hline \multicolumn{4}{|c|}{$\begin{array}{l}\text { CCPCs with prior-year taxable income between } \$ 500,000 \text { and } \\
\$ 800,000 \text { or with prior-year taxable capital employed in } \\
\text { Canada between } \$ 10 \text { million and } \$ 50 \text { million: }\end{array}$} \\
\hline Expenditures up to expenditure limit ${ }^{b}$ & $35 \%$ & $100 \%$ & $40 \%$ \\
\hline Expenditures over expenditure limit & $20 \%$ & - & - \\
\hline $\begin{array}{l}\text { CCPCs with prior-year taxable income of } \$ 800,000 \text { or } n \\
\text { with prior-year taxable capital employed in Canada of } \\
\$ 50 \text { million or more or non-CCPCs }\end{array}$ & $20 \%$ & - & - \\
\hline
\end{tabular}

Source: Department of Finance (2010a).

\section{Notes:}

${ }^{a}$ Expenditure limit is generally $\$ 3$ million per annum.

b Expenditure limit for CCPCs is phased out for prior-year taxable income between $\$ 500,000$ and $\$ 800,000$ and for prior-year taxable capital employed in Canada between $\$ 10$ million and $\$ 50$ million. 
Table 3.B-3: $\quad$ Summary of provincial indirect measures for Innovation

\begin{tabular}{|c|c|c|c|c|}
\hline $\begin{array}{l}\text { Programs and designated } \\
\text { sites }\end{array}$ & Eligible activities $^{\mathrm{a}}$ & $\begin{array}{l}\text { Tax credit } \\
\text { base }\end{array}$ & $\begin{array}{l}\text { Refundable tax } \\
\text { credit rate }^{b}\end{array}$ & Duration \\
\hline \multirow{3}{*}{$\begin{array}{l}\text { Biotechnology } \\
\text { Development Centres } \\
\text { (CDB) } \\
\text { - Laval } \\
\text { - Lévis } \\
\text { - Saint-Hyacinthe } \\
\text { - Sherbrooke }\end{array}$} & \multirow{3}{*}{$\begin{array}{l}\text { Innovative activities in } \\
\text { biotechnologies: } \\
\text { - Human health, animal } \\
\text { plant } \\
\text { agrobiotechnology, the } \\
\text { environment, and } \\
\text { human or animal } \\
\text { nutrition }\end{array}$} & $\begin{array}{l}\text { Wage paid to } \\
\text { an eligible } \\
\text { employee }\end{array}$ & $\begin{array}{l}30 \% \text { (up to } \\
\$ 11,250 \text { per } \\
\text { employee) }\end{array}$ & $\begin{array}{l}\text { Up to } 10 \text { years } \\
\text { (not to extend } \\
\text { beyond Dec. } \\
\text { 31, 2013) }\end{array}$ \\
\hline & & Eligible asset & $30 \%^{c}$ & $\begin{array}{l}3 \text { years } \\
\text { (acquisition) } \\
5 \text { years } \\
\text { (rental) }\end{array}$ \\
\hline & & $\begin{array}{l}\text { Special } \\
\text { facilities }\end{array}$ & $30 \%^{c}$ & $\begin{array}{l}5 \text { years } \\
\text { (rental) }\end{array}$ \\
\hline $\begin{array}{l}\text { Information technology } \\
\text { development centres } \\
\text { (CDTI) })^{\text {e }} \\
\quad \text { - Laval } \\
\text { - Montréal }\end{array}$ & $\begin{array}{l}\text { Innovative projects in } \\
\text { information technology } \\
\text { sectors }\end{array}$ & $\begin{array}{l}\text { Wage paid to } \\
\text { an eligible } \\
\text { employee }\end{array}$ & $\begin{array}{l}40 \% \text { (up to } \\
\$ 15,000 \text { per } \\
\text { employee) }\end{array}$ & $\begin{array}{l}\text { Up to } 10 \text { years } \\
\text { (not to extend } \\
\text { beyond Dec. } \\
31,2013 \text { ) }\end{array}$ \\
\hline $\begin{array}{l}\text {-Sherbrooke } \\
\text { —Québec City } \\
\text { — Gatineau }\end{array}$ & & $\begin{array}{l}\text { Specialized } \\
\text { equipment }\end{array}$ & $\begin{array}{l}40 \% \\
\text { (acquisition or } \\
\text { rental) }\end{array}$ & \\
\hline
\end{tabular}

\begin{tabular}{|c|c|c|c|c|}
\hline $\begin{array}{l}\text { New economy centres } \\
\text { (CNE) }^{\text {d }} \\
-\quad \text { All regions of } \\
\text { Québec }\end{array}$ & $\begin{array}{l}\text { Innovative activities in: } \\
\text { - ICT } \\
\text { - Production } \\
\text { technologies } \\
\text { - Biotechnologies } \\
\text { - Materials technology } \\
\text { - Services of a scientific } \\
\text { or technological nature }\end{array}$ & $\begin{array}{l}\text { Wage paid to } \\
\text { an eligible } \\
\text { employee }\end{array}$ & $\begin{array}{l}40 \% \text { (up to } \\
\$ 15500)\end{array}$ & $\begin{array}{l}\text { Up to } 10 \text { years } \\
\text { (not to extend } \\
\text { beyond Dec. } \\
31,2013 \text { ) }\end{array}$ \\
\hline $\begin{array}{c}\text { Innovation Centres }{ }^{\mathrm{df}} \\
-\quad \text { Montréal }^{-} \\
-\quad \text { Québec }\end{array}$ & Same as for CNE & $\begin{array}{l}\text { Increase in } \\
\text { payroll } \\
\text { attributable to } \\
\text { eligible } \\
\text { employees of } \\
\text { corporation } \\
\text { that moves } \\
\text { into a } \\
\text { designated } \\
\text { site }\end{array}$ & $40 \%$ & $\begin{array}{l}\text { Up to } 5 \text { years } \\
\text { (not to extend } \\
\text { beyond Dec. } \\
31,2006 \text { ) }\end{array}$ \\
\hline
\end{tabular}

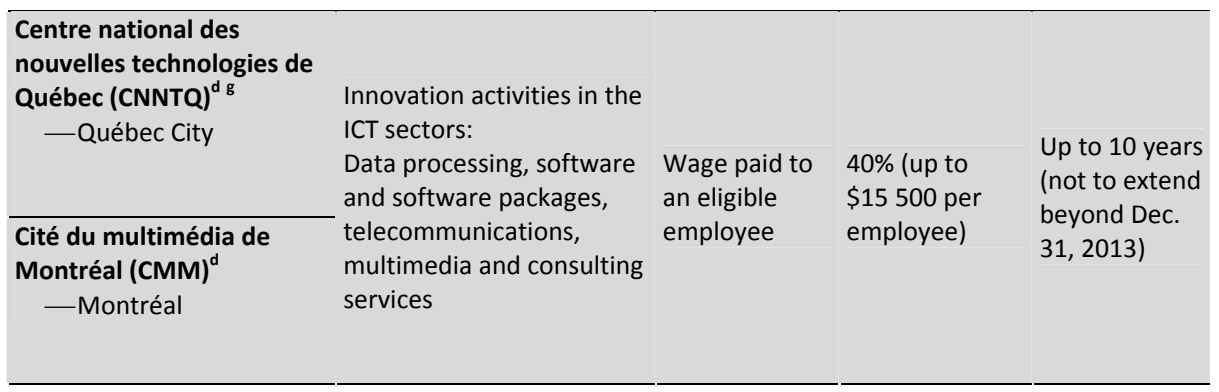




\begin{tabular}{|c|c|c|c|c|}
\hline $\begin{array}{l}\text { Programs and designated } \\
\text { sites }\end{array}$ & Eligible activities $^{a}$ & $\begin{array}{l}\text { Tax credit } \\
\text { base }\end{array}$ & $\begin{array}{l}\text { Refundable tax } \\
\text { credit rate }^{b}\end{array}$ & Duration \\
\hline $\begin{array}{c}\text { E-Commerce Place }(\mathrm{CCE})^{\mathrm{d} g} \\
-\quad \text { Montréal }\end{array}$ & $\begin{array}{l}\text { Section 1: Development } \\
\text { and supply of products } \\
\text { and services relating to } \\
\text { E-Business } \\
\text { Section 2: Operation of } \\
\text { E-Business solutions }\end{array}$ & $\begin{array}{l}\text { Wage paid to } \\
\text { an eligible } \\
\text { employee }\end{array}$ & $\begin{array}{l}35 \% \text { (up to } \$ 12 \\
500 \text { per } \\
\text { employee) }^{\mathrm{h}}\end{array}$ & $\begin{array}{l}\text { Up to } 10 \text { years } \\
\text { (not to extend } \\
\text { beyond Dec. } \\
31,2013 \text { ) }\end{array}$ \\
\hline
\end{tabular}

Sources: Ministère des Finances du Québec (2003a); Investissement Québec: http://www.investQuébec.com/fr/index.aspx?page=1287 (February 17, 2012); compiled by the author.

\section{Notes:}

${ }^{a}$ Corporations must obtain an eligibility attestation issued by Investissement Québec.

${ }^{b}$ According to the usual rules applicable to refundable tax credits, the amount of salaries must be reduced by the amount of any government assistance, any non-government assistance and any gain or advantage attributable to such salaries.

c The tax credit rate was reduced from $40 \%$ to $30 \%$ by the $2003-2004$ Budget Speech.

d The program was abolished by the 2003-2004 Budget Speech of June 12, 2003. However, the government decided to allow companies who benefited from these measures before that date to continue to claim the tax credits granted as prescribed before June 12, 2003, provided for these companies to have obtained, before June 12,2003 , an eligibility certificate issued by Investissement Québec.

Introduced by the Budget speech of Mar. 25, 1997.

${ }^{\mathrm{f}}$ Introduced by the Budget speech of Mar. 19, 2002.

${ }^{g}$ Introduced by the Budget speech of Nov. 1, 2001.

${ }^{\mathrm{h}}$ For the first five years of operation the rate of the tax credit regarding eligible salaries is equal to $35 \%$. Beginning in the sixth year of operation: the rate of the tax credit can vary depending on whether or not the eligible corporation created a sufficient number of jobs in Québec. 
Table 3.B-4: Interprovincial comparison of the main tax credits for R\&D

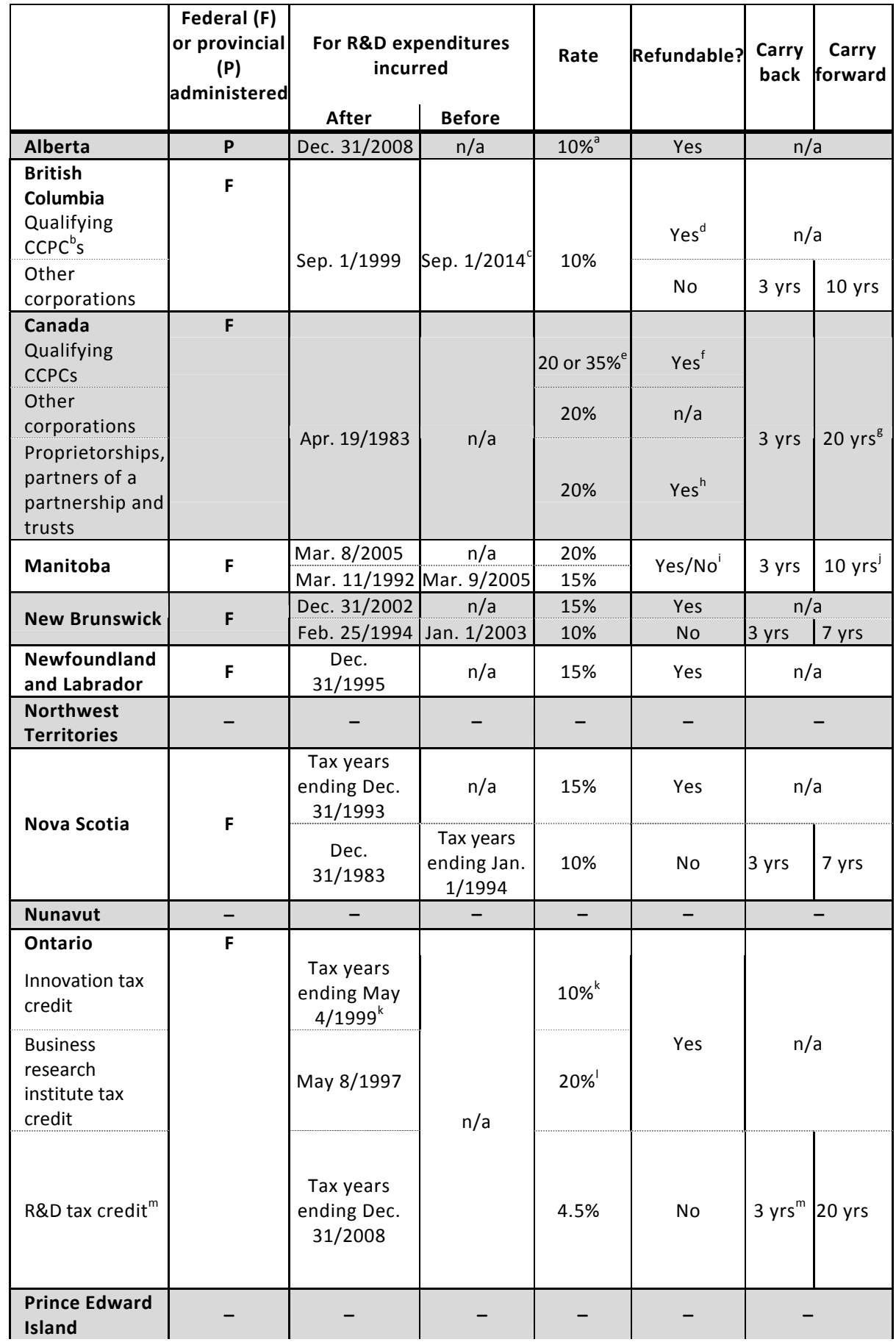




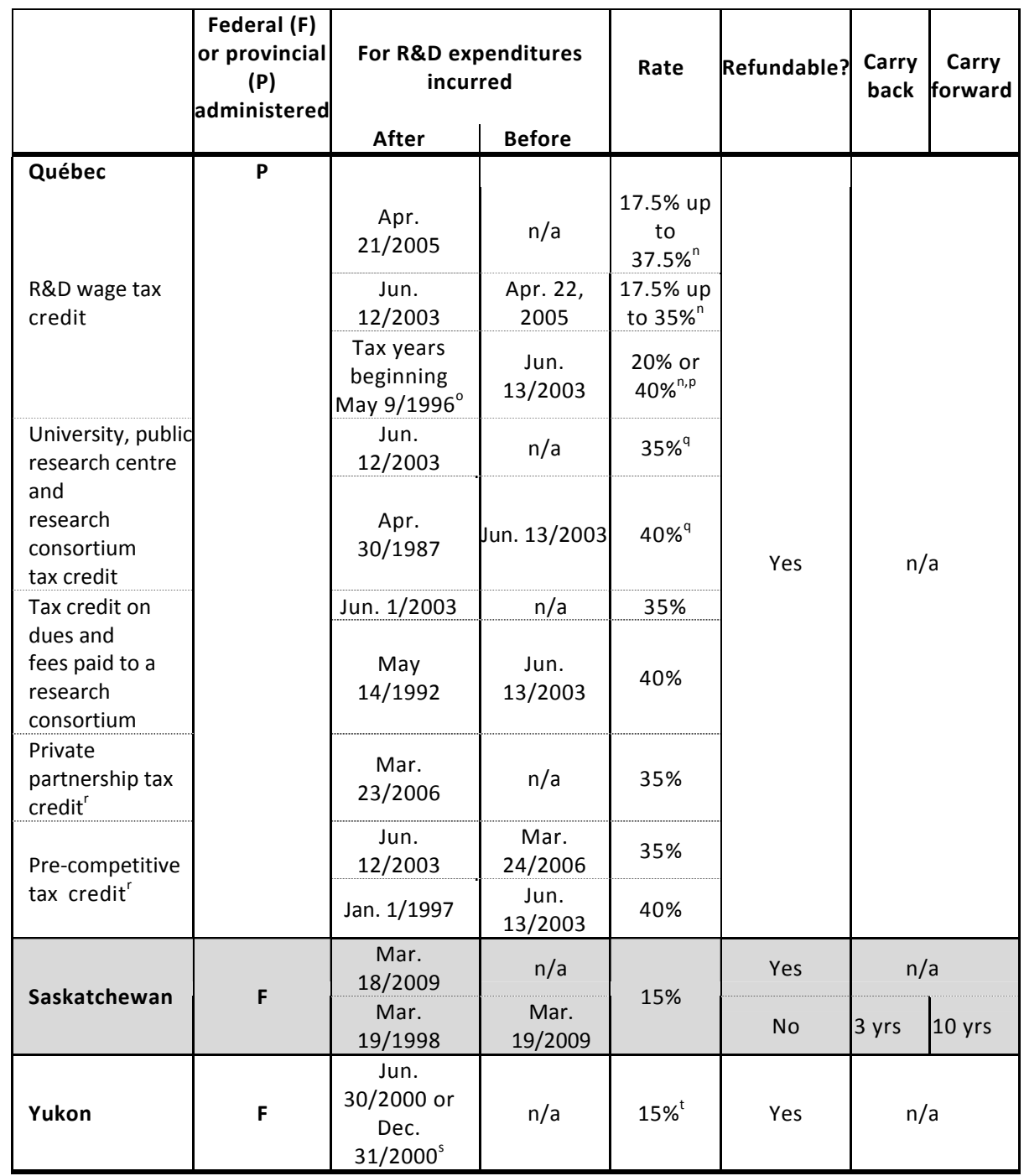

Sources: PWC, 2011, SR\&ED Tax clips: 2011 Provincial and territorial R\&D tax credits, http://www.pwc.com/ca/en/sred/tax-clips/2011-provincial-territorial-r-d-tax-credits.jhtml (Feb. 17, 2012); Revenue Canada: http://www.cra-arc.gc.ca/txcrdt/sred-rsde/Inks-eng.html (Feb. 17, 2012); Madore (1989); compiled by the author.

\section{Notes:}

a Alberta's credit equals $10 \%$ of eligible SR\&ED expenditures to a maximum expenditure level of $\$ 4$ million (maximum credit is $\$ 400,000$ ).

${ }^{\mathrm{b}}$ Canadian-Controlled Private Corporations.

c British Columbia extended its SR\&ED tax from August 31, 2004 to August 31, 2009 and then to August 31, 2014. 
d British Columbia's refundable R\&D tax credit is limited to $10 \%$ of the lesser of: (a) eligible British Columbia R\&D expenditures and (b) the federal R\&D expenditure limit (i.e., \$3 million or less for taxation years ending after February 25, 2008).

e For small Canadian-controlled private corporations with taxable income of up to $\$ 500,000$ and taxable capital of up to $\$ 10$ million can claim a refundable investment tax credit (ITC) of $35 \%$ of qualifying current and capital SR\&ED expenditures, to a maximum of $\$ 3$ million of expenditures per year. Over the $\$ 3$ million SR\&ED expenditure limit, the credit rate is reduced to $20 \%$, of which $40 \%$ may be refundable. For large Canadian corporations and foreign controlled corporations, regardless of size or whether they are public or private, the rate of the tax credit is $20 \%$ and is non-refundable.

${ }^{f}$ CCPCscan claim $100 \%$ of current expenditures at the $35 \%$ rate for the first $\$ 3$ million of current SR\&ED expenditure. For any SR\&ED expenditure on excess of the $\$ 3$ million threshold, $40 \%$ is refundable on the reduced rate of $20 \%$.

${ }^{\mathrm{g}}$ The federal government extended the carry-forward period from 10 years to 20, for 1998 and later taxation years.

${ }^{\mathrm{h}}$ Partially refundable (40\%) for in-house R\&D expenditures.

'Manitoba's 20\% credit is:

_ fully refundable for eligible expenditures incurred after 2009 by a corporation with a permanent establishment in Manitoba and that carries on research and development in Manitoba under an eligible contract with a qualifying research institute;

_ partially refundable (25\% in 2011 and 50\% after 2011) for in-house R\&D expenditures.

${ }^{j}$ Manitoba extended the carry-forward period from 7 years to 10, for 2004 and later taxation years.

${ }^{k}$ Ontario corporations qualify for the refundable credit on eligible expenditures incurred up to the expenditure limit ( $\$ 3$ million or less) and must be shared by associated corporations. The expenditure limit is reduced when:

— the previous year's taxable capital of the worldwide associated group is between $\$ 25$ million and $\$ 50$ million; or

_ the previous year's taxable income of the worldwide associated group is between $\$ 500,000$ and $\$ 800,000$.

$100 \%$ of current expenditures and $40 \%$ of capital expenditures are eligible for the credit.

The OITC was originally available to Canadian-controlled private corporations effective for taxation years ending after December 31, 1994. For taxation years ending after May 4, 1999, the credit is extended to all public and private corporations and is no longer limited to the amount eligible for the federal $35 \%$ R\&D tax credit.

'Ontario's credit is calculated as $20 \%$ of qualifying payments (up to $\$ 20$ million annually on an associated basis) to Ontario eligible research institutes. The maximum annual credit is $\$ 4$ million.

${ }^{m}$ For taxation years ending after 2008, the R\&D tax credit replaces Ontario's deduction for the portion of the federal investment tax credit relating to qualifying Ontario R\&D expenditures. The credit can be carried back only to taxation years ending after 2008 .

${ }^{n}$ Québec Canadian-controlled corporations with less than $\$ 50$ million in assets, on an associated basis, can claim the $37.5 \%$ rate on up to the spending limit of $\$ 3$ million of R\&D wages, on an associated basis. For those with assets between $\$ 50$ million and $\$ 75$ million, the $37.5 \%$ rate is gradually reduced to $17.5 \%$. The rate is $17.5 \%$ for all other taxpayers. The rate increased from $35 \%$ to $37.5 \%$ on R\&D expenditures incurred after April 21, 2005. For expenditures incurred before June 13, 2003, the 35\% rate was $40 \%$ and the $17.5 \%$ was $20 \% .50 \%$ of payments to arm's length subcontractors are eligible for the credit. All thresholds are in respect of the previous year, on a worldwide associated basis. 
${ }^{\circ}$ Before this date, Québec's R\&D wage tax credit was subject to different eligibility criteria, rates and restrictions.

${ }^{p}$ Corporations that qualified for Québec's R\&D wage tax credit at the $40 \%$ rate (i.e., Canadian-controlled corporations with assets under $\$ 25$ million) qualified for an additional $15 \%$ tax credit based on the increase in all R\&D expenditures over the average expenditures in the last three taxation years. This additional credit was to have been available to eligible corporations until taxation years beginning before July 1, 2004, but its expiry was accelerated to taxation years beginning after June 12, 2003.

q Québec's 35\% (40\% before June 12, 2003) credit is available on $80 \%$ of payments to certain eligible entities (e.g., universities and public research centres).

'The Québec tax credit for private partnerships replaced the pre-competitive research tax credit.

${ }^{5}$ The credit applies to eligible expenditures incurred in the Yukon after June 30, 2000 for corporations, and after December 31, 2000 for individuals.

${ }^{t}$ Yukon's rate is $20 \%$ on R\&D expenditures made to the Yukon College. 


\section{C. Costs of R\&D and Innovation Measures}

Table 3.C-5: Estimated cost of fiscal incentives for R\&D and innovation, Québec, 1997-2007

\begin{tabular}{|c|c|c|c|c|c|c|c|c|c|c|c|}
\hline & \multicolumn{11}{|c|}{ (\$ millions) } \\
\hline & 1997 & 1998 & 1999 & 2000 & 2001 & 2002 & 2003 & 2004 & 2005 & 2006 & 2007 \\
\hline \multicolumn{12}{|l|}{ R\&D } \\
\hline \multicolumn{12}{|l|}{$\begin{array}{l}\text { SR\&DE Tax } \\
\text { credit }\end{array}$} \\
\hline - Salaries & 319 & 326 & 383 & 372 & 506 & 566 & 591 & 581 & 611 & 654 & 697 \\
\hline - University & 7 & 6 & 6 & 7 & 8 & 7 & 7 & 5 & 5 & 6 & 4 \\
\hline - Other & 21 & 19 & 29 & 28 & 24 & 25 & 25 & 32 & 28 & 35 & 35 \\
\hline Superdeductions & - & - & - & 55 & 44 & - & - & - & - & - & - \\
\hline $\begin{array}{l}\text { Increase in R\&D } \\
\text { expenditures }\end{array}$ & - & - & $f$ & 25 & 45 & 41 & 46 & 13 & - & - & - \\
\hline Total R\&D & 347 & 351 & 418 & 487 & 627 & 639 & 669 & 631 & 538 & 664 & 695 \\
\hline \multicolumn{12}{|l|}{ Innovation $^{\mathrm{a}}$} \\
\hline Design $^{b}$ & 6 & 6 & 7 & 8 & 8 & 8 & 7 & 6 & 6 & 8 & 10 \\
\hline $\begin{array}{l}\text { Production of } \\
\text { multimedia } \\
\text { titles }^{c}\end{array}$ & $f$ & 13 & 12 & 19 & 22 & 23 & 22 & 24 & 36 & 44 & 60 \\
\hline CDTI & $f$ & 5 & 14 & 21 & 22 & 22 & 16 & 15 & 11 & 7 & 7 \\
\hline CMM & $f$ & $f$ & 11 & 23 & 35 & 35 & 30 & 31 & 39 & 44 & 44 \\
\hline CNNTQ & $f$ & $f$ & $f$ & 6 & 12 & 14 & 13 & 12 & 11 & 12 & 15 \\
\hline CNE & $f$ & $f$ & $f$ & 5 & 15 & 22 & 39 & 44 & 46 & 48 & 45 \\
\hline CCE & $f$ & $f$ & - & 12 & 35 & 49 & 66 & 72 & 73 & 74 & 75 \\
\hline CDB & & & - & - & $f$ & $f$ & $f$ & $f$ & $f$ & $f$ & $f$ \\
\hline E-business ${ }^{d}$ & - & - & - & - & $f$ & 9 & 22 & 32 & 42 & 38 & 21 \\
\hline $\begin{array}{l}\text { Innovation } \\
\text { centres }\end{array}$ & - & - & - & - & - & $f$ & $f$ & 2 & $f$ & 2 & 3 \\
\hline Innovation & 6 & 24 & 44 & 94 & 152 & 186 & 215 & 238 & 264 & 279 & 289 \\
\hline TOTAL & 353 & 375 & 462 & 581 & 779 & 825 & 884 & 869 & 908 & 974 & 1025 \\
\hline
\end{tabular}

Sources: Estimates for 1997-1998 (Ministère des Finances du Québec (2003b)), estimates for 19992002 (Ministère des Finances du Québec (2005)), estimates for 2003 (Ministère des Finances du Québec (2008)) estimates for 2004 (Ministère des Finances du Québec (2009)) and estimates for 2005-2007 (Ministère des Finances du Québec (2010)).

\section{Notes:}

(-) The measure did not apply that year.

(f) The tax cost is less than $\$ 2$ million.

Tax expenditures represent an estimate, which means, in general, that their cost is calculated from real tax statistics from the ministère du Revenu, where available, or, otherwise, from other sources and using certain assumptions.

a Certain fiscal incentives appearing under "Innovation" cover activities which do not necessarily respect the definition of innovation in the strict sense of the Oslo Manual. The term "New economy" is used in budgetary documents of the Ministère des Finances du Québec to designate these measures.

${ }^{b}$ The refundable tax credit for design was introduced in 1994 and applies regarding certain expenditures that an eligible corporation incurs in relation to eligible design activities. 
${ }^{c}$ The refundable tax credit for the production of multimedia titles was introduced in 1996 depends on the eligible labour expenditures incurred in the production of eligible multimedia titles, or, since March 30,2010 , in the production of eligible related titles.

d The refundable tax credit for e-business activities carried out in certain designated sites was introduced on November 1, 2001 and eliminated in the June 12, 2003 Budget Speech.

Table 3.C-6: Cost of direct programs for R\&D and innovation, 2000-2001 to 2003-2004

\begin{tabular}{|c|c|c|c|c|}
\hline & \multicolumn{4}{|c|}{$(\$)$} \\
\hline & 2000-2001 & 2001-2002 & 2002-2003 & 2003-2004 \\
\hline Direct assistance programs for R\&D & 384644979 & 372600667 & 347747119 & 491744996 \\
\hline Enterprises & 22446835 & 23612667 & 26386331 & 32863299 \\
\hline Higher Education & 228519351 & 286217988 & 236520124 & 376812471 \\
\hline Hospitals and health agencies & 66680798 & 40651565 & 48354345 & 32768398 \\
\hline Provincial research organizations & 866004 & 787700 & 259600 & 6784954 \\
\hline Other organizations & 66131991 & 21330747 & 36226719 & 42515874 \\
\hline Direct assistance programs for innovation & 48827027 & 121859021 & 164700872 & 79543952 \\
\hline Enterprises & 29381991 & 117838714 & 150358709 & 67004822 \\
\hline Higher Education & 6090345 & 1233869 & 6339075 & 5850763 \\
\hline Hospitals and health agencies & 255550 & 276995 & 41300 & 6326 \\
\hline Provincial research organizations & 138740 & 179720 & 81293 & 357564 \\
\hline Other organizations & 12960401 & 2329723 & 7880495 & 6324477 \\
\hline
\end{tabular}

Source:

Survey of research, science, technology and innovation expenditures in the Québec public administration (2000-2001 to 2003-2004). 
Table 3.C-7: $\quad$ Cost of direct programs for R\&D, federal Government, 2003-2004 to 2008-2009

\begin{tabular}{lrrrrrr}
\hline & \multicolumn{7}{c}{ (\$ millions) } \\
\hline & $2003-$ & $2004-$ & $2005-$ & $2006-$ & $2007-$ & $2008-$ \\
& 2004 & 2005 & 2006 & 2007 & 2008 & 2009 \\
\hline Direct assistance programs for R\&D & $\mathbf{7 7 7}$ & $\mathbf{7 8 9}$ & $\mathbf{8 6 0}$ & $\mathbf{8 8 2}$ & $\mathbf{9 2 9}$ & $\mathbf{1 0 0 9}$ \\
Enterprises & 221 & 214 & 203 & 217 & 240 & 274 \\
Higher Education & 513 & 545 & 614 & 630 & 632 & 688 \\
Other performer & 43 & 30 & 43 & 35 & 57 & 47 \\
\hline
\end{tabular}

Source: Statistics Canada. Table 358-0150 - Federal extramural expenditures on science and technology and its components, by type of science, performing sector, type of payment, Canada, provinces and territories, annual (dollars), CANSIM, Using http://estat.statcan.gc.ca/cgi-win/cnsmcgi.exe?Lang=E\&EST-Fi=EStat/English/CII_1-eng.htm.

Table 3.C-8: Total expenditures of provincial governments on R\&D, 1994-1995 to 2002-2003

\begin{tabular}{lrrrrrrrrr}
\hline \multicolumn{10}{c}{ (\$ millions) } \\
\hline Province & $\mathbf{1 9 9 4 -}$ & $\mathbf{1 9 9 5 -}$ & $\mathbf{1 9 9 6 -}$ & $\mathbf{1 9 9 7 -}$ & $\mathbf{1 9 9 8 -}$ & $\mathbf{1 9 9 9 -}$ & $\mathbf{2 0 0 0 -}$ & $\mathbf{2 0 0 1 -}$ & $\begin{array}{r}\mathbf{2 0 0 2}- \\
\mathbf{2 0 0 3 p}\end{array}$ \\
\hline Québec & $\mathbf{1 9 9 5}$ & $\mathbf{1 9 9 6}$ & $\mathbf{1 9 9 7}$ & $\mathbf{1 9 9 8}$ & $\mathbf{1 9 9 9}$ & $\mathbf{2 0 0 0 r}$ & $\mathbf{2 0 0 1 r}$ & $\mathbf{2 0 0 2 r}$ & $\mathbf{2 0 0}$ \\
Ontario & 230.5 & 218.3 & 216.2 & 206.7 & 213.3 & 455.0 & 429.4 & 426.4 & 413.0 \\
Manitoba & 250.4 & 250.9 & 210.6 & 210.2 & 213.6 & 280.8 & 421.0 & 443.5 & 488.7 \\
Saskatchewan & 11.8 & 10.6 & 10.2 & 7.1 & 15.1 & 14.7 & 18.0 & 18.7 & 19.4 \\
Alberta & 102.7 & 31.6 & 27.9 & 55.4 & 56.7 & 45.9 & 76.3 & 71.8 & n.a \\
British & 101.9 & 110.5 & 126.5 & 157.4 & 173.2 & 198.1 & 245.6 & 268.0 \\
Columbia & 72.6 & 78.0 & 89.3 & 88.7 & 72.8 & 72.7 & 199.9 & 93.6 & 106.1 \\
\hline
\end{tabular}

Source: Statiscs Canada, Scientific and technological (S\&T) activities of provincial governments, 199495 to 2002-03, 88-001-x2004006, June 2004.

Note: $\quad$ : revised; p: preliminary. 


\section{D. Net Cost of R\&D Expenditures for Business Enterprises}

Table 3.D-9: Net cost of $\$ 100,000$ R\&D expenditures incurred in Québec by an SME in the manufacturing sector, 2003 tax rates

Example 1: $\quad$ A corporation incurs $R \& D$ expenditures of $\$ 100,000$, i.e. $\$ 80,000$ for the salary of a researcher and $\$ 20,000$ for materials.

Example 2: $\quad$ A corporation incurs $R \& D$ expenditures of $\$ 100,000$, i.e. $\$ 60,000$ for the salary of a researcher, $\$ 30,000$ for materials and $\$ 10,000$ for equipment.

\begin{tabular}{|c|c|c|c|}
\hline & & Example 1 & Example 2 \\
\hline & Expenditures & & \\
\hline \multirow[t]{3}{*}{ A } & Salaries & 80,000 & 60,000 \\
\hline & Materials & 20,000 & 30,000 \\
\hline & Equipment & & 10,000 \\
\hline \multirow[t]{6}{*}{ B } & Total R\&D expenditures & 100,000 & 100,000 \\
\hline & $\begin{array}{l}\text { Québec tax credit } \\
(\text { A X 35\%) }\end{array}$ & 28,000 & 21,000 \\
\hline & Federal tax credit & & \\
\hline & Total expenditures (B) & 100,000 & 100,000 \\
\hline & Overhead (A X 65\%) & 52,000 & 39,000 \\
\hline & Less Québec credit & $(28,000)$ & $(21,000)$ \\
\hline $\mathrm{C}$ & QR\&E or Qualified Research Expenditure & 124,000 & 118,000 \\
\hline $\mathrm{D}$ & $(\mathrm{C} \times 35 \%)$ & 43,400 & 41,300 \\
\hline \multirow[t]{2}{*}{$\mathrm{F}$} & Total tax credits (Québec + Federal) & 71,400 & 62,300 \\
\hline & Net cost for the corporation (B-F) & 28,600 & 37,700 \\
\hline
\end{tabular}

Notes: $\quad$ For purposes of tax credit, an SME is a Canadian-controlled private corporation whose assets in the financial statements of all the corporations in the group are less than $\$ 25,000,000$.

For purposes of the federal tax credit, an SME is a Canadian-controlled private corporation whose taxable income and taxable capital for all the corporations in the group do not exceed $\$ 300,000$ and $\$ 10,000,000$ for the preceding taxation year respectively.

Proxy method used to calculate overhead. Under this method, actual overhead is replaced by $65 \%$ of R\&D salaries. 
Table 3.D-10: Net cost of $\$ 100,000$ R\&D expenditures incurred in Québec by a large corporation or a foreign-controlled corporation in the manufacturing sector, 2003 tax rates

Example 1: $\quad$ A corporation incurs $R \& D$ expenditures of $\$ 100,000$, i.e. $\$ 80,000$ for the salary of a researcher and $\$ 20,000$ for materials.

Example 2: $\quad$ A corporation incurs $R \& D$ expenditures of $\$ 100,000$, i.e. $\$ 60,000$ for the salary of a researcher, $\$ 30,000$ for materials and $\$ 10,000$ for equipment.

\begin{tabular}{|c|c|c|c|}
\hline & & Example 1 & Example 2 \\
\hline & Expenditures & & \\
\hline \multirow[t]{3}{*}{ A } & Salaries & 80,000 & 60,000 \\
\hline & Materials & 20,000 & 30,000 \\
\hline & Equipment & & 10,000 \\
\hline \multirow[t]{6}{*}{ B } & Total R\&D expenditures & 100,000 & 100,000 \\
\hline & $\begin{array}{l}\text { Québec tax credit } \\
(\mathrm{A} \times 17.5 \%)\end{array}$ & 14,000 & 10,500 \\
\hline & Federal tax credit & & \\
\hline & Total expenditures (B) & 100,000 & 100,000 \\
\hline & Overhead (A X 65\%) & 52,000 & 39,000 \\
\hline & Less credit & $(14,000)$ & $(10,500)$ \\
\hline C & QR\&E or Qualified Research Expenditure & 138,000 & 128,500 \\
\hline D & (CX20\%) & 27,600 & 25,700 \\
\hline \multirow[t]{2}{*}{$\mathrm{F}$} & Total tax credits (Québec + Federal) & 41,600 & 36,200 \\
\hline & Net cost for the corporation (B-F) & 58,400 & 63,800 \\
\hline
\end{tabular}

Notes: For purposes of tax credit, a large corporation is a Canadian-controlled private corporation whose assets in the financial statements of all the corporations in the group exceed $\$ 25,000,000$.

For purposes of the federal tax credit, a large corporation is a Canadian-controlled private corporation whose taxable income and taxable capital for all the corporations in the group exceed $\$ 300,000$ and $\$ 10,000,000$ for the preceding taxation year respectively.

Proxy method used to calculate overhead. Under this method, actual overhead is replaced by $65 \%$ of R\&D salaries. 


\section{E. Main Statistics on R\&D and Innovation}

Table 3.E-11: Gross domestic expenditure on R\&D (GERD), percentage of GDP, Canada and Canadian provinces, 1981-2008

\begin{tabular}{|c|c|c|c|c|c|c|c|c|c|c|c|}
\hline & CA & NFL & PEI & NS & NB & QC & ON & MB & SK & $A L$ & BC \\
\hline 1981 & 1.22 & 0.74 & 0.66 & 1.14 & 0.58 & 1.02 & 1.27 & 1.00 & 0.58 & 0.88 & 0.59 \\
\hline 1982 & 1.37 & 0.86 & 0.61 & 1.18 & 0.67 & 1.12 & 1.47 & 1.15 & 0.79 & 0.92 & 0.66 \\
\hline 1983 & 1.34 & 1.17 & 0.52 & 1.40 & 0.53 & 1.08 & 1.45 & 1.26 & 0.78 & 0.80 & 0.70 \\
\hline 1984 & 1.40 & 0.93 & 0.72 & 1.40 & 0.56 & 1.24 & 1.44 & 1.20 & 0.79 & 0.82 & 0.76 \\
\hline 1985 & 1.44 & 1.04 & 0.62 & 1.36 & 0.97 & 1.49 & 1.64 & 1.08 & 0.97 & 0.93 & 0.91 \\
\hline 1986 & 1.47 & 0.84 & 1.53 & 1.34 & 0.79 & 1.42 & 1.66 & 1.03 & 0.99 & 1.10 & 0.93 \\
\hline 1987 & 1.42 & 0.90 & 0.81 & 1.18 & 0.77 & 1.46 & 1.60 & 0.92 & 0.93 & 0.96 & 0.77 \\
\hline 1988 & 1.48 & 1.09 & 0.68 & 1.77 & 1.22 & 1.49 & 1.84 & 1.05 & 0.93 & 1.03 & 0.89 \\
\hline 1989 & 1.45 & 1.10 & 0.78 & 1.44 & 1.23 & 1.57 & 1.75 & 1.07 & 0.95 & 1.02 & 0.89 \\
\hline 1990 & 1.51 & 1.12 & 0.74 & 1.39 & 1.00 & 1.70 & 1.82 & 1.09 & 0.95 & 1.07 & 0.97 \\
\hline 1991 & 1.57 & 1.11 & 0.71 & 1.36 & 0.89 & 1.86 & 1.88 & 1.18 & 1.01 & 1.08 & 0.96 \\
\hline 1992 & 1.62 & 1.15 & 0.60 & 1.29 & 0.87 & 1.98 & 1.94 & 1.15 & 1.11 & 1.04 & 1.01 \\
\hline 1993 & 1.68 & 1.14 & 0.69 & 1.34 & 0.88 & 2.04 & 2.08 & 1.20 & 1.02 & 1.03 & 0.97 \\
\hline 1994 & 1.73 & 1.05 & 0.67 & 1.42 & 0.88 & 2.08 & 2.15 & 1.20 & 0.98 & 1.10 & 1.06 \\
\hline 1995 & 1.70 & 0.94 & 0.60 & 1.37 & 0.85 & 2.10 & 2.10 & 1.09 & 0.96 & 1.06 & 1.01 \\
\hline 1996 & 1.65 & 0.98 & 0.60 & 1.32 & 0.90 & 2.12 & 2.05 & 1.04 & 0.80 & 1.02 & 0.92 \\
\hline 1997 & 1.66 & 0.98 & 0.64 & 1.26 & 0.75 & 2.10 & 2.09 & 0.91 & 0.99 & 0.98 & 0.91 \\
\hline 1998 & 1.76 & 1.06 & 0.81 & 1.45 & 0.88 & 2.22 & 2.18 & 0.96 & 0.94 & 1.10 & 0.96 \\
\hline 1999 & 1.80 & 1.04 & 0.82 & 1.47 & 0.86 & 2.33 & 2.17 & 1.20 & 1.05 & 1.00 & 1.07 \\
\hline 2000 & 1.91 & 0.99 & 1.10 & 1.47 & 0.79 & 2.54 & 2.36 & 1.15 & 1.11 & 0.91 & 1.22 \\
\hline 2001 & 2.09 & 1.00 & 1.08 & 1.45 & 0.78 & 2.77 & 2.59 & 1.30 & 1.20 & 1.05 & 1.32 \\
\hline 2002 & 2.04 & 0.93 & 0.84 & 1.48 & 1.00 & 2.79 & 2.38 & 1.24 & 1.27 & 1.14 & 1.41 \\
\hline 2003 & 2.04 & 0.95 & 1.13 & 1.42 & 0.96 & 2.78 & 2.43 & 1.21 & 1.09 & 1.12 & 1.41 \\
\hline 2004 & 2.07 & 0.89 & 1.03 & 1.50 & 0.96 & 2.76 & 2.51 & 1.30 & 1.04 & 1.19 & 1.44 \\
\hline 2005 & 2.04 & 1.22 & 1.61 & 1.49 & 1.04 & 2.67 & 2.54 & 1.40 & 1.03 & 1.10 & 1.42 \\
\hline 2006 & 2.00 & 1.01 & 1.60 & 1.59 & 1.06 & 2.80 & 2.46 & 1.24 & 1.04 & 1.09 & 1.33 \\
\hline 2007 & 1.96 & 0.92 & 1.32 & 1.53 & 1.17 & 2.72 & 2.38 & 1.22 & 0.98 & 1.05 & 1.46 \\
\hline 2008 & 1.87 & 0.88 & 1.38 & 1.51 & 1.10 & 2.61 & 2.37 & 1.11 & 0.81 & 0.99 & 1.42 \\
\hline
\end{tabular}

Source: Statistics Canada, Table 358-0001 - Gross domestic expenditures on research and development, Table 384-0002 - Gross domestic product (GDP), CANSIM (database).

Note: $\quad$ CA: Canada; NFL: Newfoundland and Labrador; PEI: Prince Edward Island; NS: Nova Scotia; NB: New Brunswick; QC: Québec; ON: Ontario; MB: Manitoba; SK: Saskatchewan; AB: Alberta; BC: British Columbia. 
Table 3.E-12: Business expenditure on R\&D (BERD), percentage of GDP, Canada and Canadian provinces, 1981-2008

\begin{tabular}{|c|c|c|c|c|c|c|c|c|c|c|c|}
\hline & CA & NFL & PEI & NS & NB & QC & ON & MB & SK & $A L$ & BC \\
\hline 1981 & 0.6 & 0.2 & 0.1 & 0.1 & 0.1 & 0.6 & 0.9 & 0.2 & 0.2 & 0.5 & 0.3 \\
\hline 1982 & 0.7 & 0.0 & 0.0 & 0.1 & 0.1 & 0.7 & 1.0 & 0.2 & 0.3 & 0.5 & 0.3 \\
\hline 1983 & 0.6 & 0.1 & 0.0 & 0.1 & 0.1 & 0.6 & 1.0 & 0.2 & 0.2 & 0.4 & 0.3 \\
\hline 1984 & 0.7 & 0.1 & 0.1 & 0.1 & 0.1 & 0.7 & 1.0 & 0.2 & 0.3 & 0.4 & 0.4 \\
\hline 1985 & 0.7 & 0.1 & 0.1 & 0.2 & 0.3 & 0.8 & 1.1 & 0.1 & 0.3 & 0.4 & 0.4 \\
\hline 1986 & 0.8 & 0.1 & 0.7 & 0.2 & 0.2 & 0.8 & 1.1 & 0.2 & 0.3 & 0.4 & 0.5 \\
\hline 1987 & 0.8 & 0.1 & 0.2 & 0.2 & 0.2 & 0.9 & 1.1 & 0.2 & 0.3 & 0.4 & 0.4 \\
\hline 1988 & 0.8 & 0.1 & 0.1 & 0.4 & 0.6 & 0.8 & 1.1 & 0.2 & 0.2 & 0.4 & 0.4 \\
\hline 1989 & 0.7 & 0.1 & 0.1 & 0.2 & 0.6 & 0.8 & 1.0 & 0.2 & 0.2 & 0.4 & 0.4 \\
\hline 1990 & 0.8 & 0.1 & 0.1 & 0.2 & 0.3 & 0.9 & 1.0 & 0.2 & 0.2 & 0.5 & 0.5 \\
\hline 1991 & 0.8 & 0.1 & 0.1 & 0.1 & 0.2 & 1.0 & 1.0 & 0.3 & 0.3 & 0.5 & 0.4 \\
\hline 1992 & 0.8 & 0.1 & 0.0 & 0.2 & 0.2 & 1.0 & 1.1 & 0.3 & 0.3 & 0.4 & 0.5 \\
\hline 1993 & 0.9 & 0.1 & 0.1 & 0.2 & 0.3 & 1.1 & 1.2 & 0.4 & 0.3 & 0.5 & 0.5 \\
\hline 1994 & 1.0 & 0.1 & 0.1 & 0.3 & 0.3 & 1.2 & 1.3 & 0.4 & 0.3 & 0.6 & 0.6 \\
\hline 1995 & 1.0 & 0.1 & 0.1 & 0.3 & 0.3 & 1.3 & 1.3 & 0.4 & 0.3 & 0.5 & 0.6 \\
\hline 1996 & 1.0 & 0.2 & 0.1 & 0.3 & 0.4 & 1.3 & 1.3 & 0.3 & 0.2 & 0.5 & 0.5 \\
\hline 1997 & 1.0 & 0.1 & 0.1 & 0.3 & 0.2 & 1.3 & 1.3 & 0.3 & 0.3 & 0.5 & 0.5 \\
\hline 1998 & 1.1 & 0.2 & 0.1 & 0.3 & 0.2 & 1.4 & 1.4 & 0.3 & 0.3 & 0.6 & 0.5 \\
\hline 1999 & 1.1 & 0.1 & 0.1 & 0.3 & 0.2 & 1.4 & 1.4 & 0.5 & 0.3 & 0.4 & 0.6 \\
\hline 2000 & 1.2 & 0.1 & 0.1 & 0.3 & 0.2 & 1.6 & 1.6 & 0.4 & 0.2 & 0.4 & 0.7 \\
\hline 2001 & 1.3 & 0.1 & 0.2 & 0.4 & 0.2 & 1.8 & 1.7 & 0.5 & 0.3 & 0.5 & 0.8 \\
\hline 2002 & 1.2 & 0.1 & 0.1 & 0.3 & 0.3 & 1.7 & 1.5 & 0.4 & 0.3 & 0.5 & 0.8 \\
\hline 2003 & 1.2 & 0.2 & 0.2 & 0.3 & 0.3 & 1.7 & 1.5 & 0.4 & 0.2 & 0.5 & 0.8 \\
\hline 2004 & 1.2 & 0.2 & 0.2 & 0.3 & 0.3 & 1.6 & 1.5 & 0.5 & 0.3 & 0.6 & 0.8 \\
\hline 2005 & 1.1 & 0.4 & 0.3 & 0.3 & 0.4 & 1.5 & 1.5 & 0.5 & 0.3 & 0.5 & 0.8 \\
\hline 2006 & 1.1 & 0.4 & 0.3 & 0.3 & 0.4 & 1.7 & 1.5 & 0.4 & 0.4 & 0.6 & 0.7 \\
\hline 2007 & 1.1 & 0.3 & 0.3 & 0.3 & 0.4 & 1.7 & 1.4 & 0.4 & 0.4 & 0.6 & 0.8 \\
\hline 2008 & 1.0 & 0.3 & 0.3 & 0.3 & 0.4 & 1.5 & 1.3 & 0.3 & 0.2 & 0.5 & 0.8 \\
\hline
\end{tabular}

Source: Statistics Canada, Table 358-0001 - Gross domestic expenditures on research and development, Table 384-0002 - Gross domestic product (GDP), CANSIM (database).

Note: $\quad$ CA: Canada; NFL: Newfoundland and Labrador; PEl: Prince Edward Island; NS: Nova Scotia; NB: New Brunswick; QC: Québec; ON: Ontario; MB: Manitoba; SK: Saskatchewan; AB: Alberta; BC: British Columbia. 
Table 3.E-13: GERD, percentage of GDP, Québec, Canada and OECD countries, 1997-2008

\begin{tabular}{|c|c|c|c|c|c|c|c|c|c|c|c|c|}
\hline & 1997 & 1998 & 1999 & 2000 & 2001 & 2002 & 2003 & 2004 & 2005 & 2006 & 2007 & 2008 \\
\hline Germany & 2.24 & 2.27 & 2.40 & 2.45 & 2.46 & 2.49 & 2.52 & 2.49 & 2.49 & 2.53 & 2.53 & 2.68 \\
\hline Australia & .. & 1.51 & .. & 1.56 & .. & 1.64 & .. & 1.72 & .. & 2.00 & 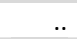 & 2.21 \\
\hline Austria & 1.69 & 1.77 & 1.88 & 1.91 & 2.03 & 2.14 & 2.26 & 2.26 & 2.48 & 2.46 & 2.52 & 2.67 \\
\hline Belgium & 1.87 & 1.90 & 1.96 & 2.00 & 2.11 & 1.94 & 1.88 & 1.86 & 1.83 & 1.86 & 1.90 & 1.96 \\
\hline Canada & 1.66 & 1.76 & 1.80 & 1.91 & 2.09 & 2.04 & 2.04 & 2.07 & 2.04 & 2.00 & 1.96 & 1.87 \\
\hline Québec & 2.10 & 2.22 & 2.33 & 2.54 & 2.77 & 2.79 & 2.78 & 2.76 & 2.67 & 2.80 & 2.72 & 2.61 \\
\hline Korea & 2.48 & 2.34 & 2.25 & 2.39 & 2.59 & 2.40 & 2.49 & 2.68 & 2.79 & 3.01 & 3.21 & 3.36 \\
\hline Denmark & 1.92 & 2.04 & 2.18 & .. & 2.39 & 2.51 & 2.58 & 2.48 & 2.46 & 2.48 & 2.58 & 2.87 \\
\hline Spain & 0.79 & 0.87 & 0.86 & 0.91 & 0.92 & 0.99 & 1.05 & 1.06 & 1.12 & 1.20 & 1.27 & 1.35 \\
\hline $\begin{array}{l}\text { United } \\
\text { States }\end{array}$ & 2.58 & 2.62 & 2.66 & 2.74 & 2.76 & 2.62 & 2.61 & 2.54 & 2.57 & 2.61 & 2.67 & 2.79 \\
\hline Finland & 2.69 & 2.86 & 3.21 & 3.38 & 3.38 & 3.37 & 3.44 & 3.45 & 3.48 & 3.48 & 3.47 & 3.72 \\
\hline France & 2.19 & 2.14 & 2.16 & 2.15 & 2.20 & 2.23 & 2.17 & 2.15 & 2.10 & 2.10 & 2.07 & 2.11 \\
\hline Greece & 0.51 & .. & 0.67 &.. & 0.65 & .. & 0.57 & 0.55 & 0.59 & 0.58 & 0.59 & .. \\
\hline Hungary & 0.72 & 0.68 & 0.69 & 0.80 & 0.95 & 1.00 & 0.93 & 0.87 & 0.95 & 1.00 & 0.97 & 1.00 \\
\hline Ireland & 1.29 & 1.25 & 1.19 & 1.14 & 1.11 & 1.10 & 1.17 & 1.23 & 1.25 & 1.25 & 1.29 & 1.45 \\
\hline Iceland & 1.89 & 2.08 & 2.39 & 2.76 & 3.08 & 2.95 & 2.82 & .. & 2.77 & 2.99 & 2.68 & 2.64 \\
\hline Italy & 1.05 & 1.07 & 1.04 & 1.07 & 1.11 & 1.13 & 1.11 & 1.10 & 1.09 & 1.13 & 1.18 & 1.23 \\
\hline Japan & 2.84 & 2.95 & 2.96 & 2.99 & 3.07 & 3.17 & 3.20 & 3.17 & 3.32 & 3.40 & 3.44 & 3.44 \\
\hline Luxembourg & .. & .. & .. & 1.71 & .. & .. & 1.65 & 1.63 & 1.56 & 1.66 & 1.58 & 1.56 \\
\hline Mexico & 0.34 & 0.38 & 0.43 & 0.37 & 0.39 & 0.40 & 0.40 & 0.40 & 0.41 & 0.39 & 0.37 & .. \\
\hline Norway & 1.64 & .. & 1.65 &. & 1.60 & 1.66 & 1.71 & 1.59 & 1.52 & 1.52 & 1.62 & 1.61 \\
\hline $\begin{array}{l}\text { New } \\
\text { Zealand }\end{array}$ & 1.10 &.. & 1.01 & .. & 1.15 & .. & 1.17 & .. & 1.14 & .. & 1.17 & .. \\
\hline Netherlands & 2.04 & 1.94 & 2.02 & 1.90 & 1.88 & 1.88 & 1.92 & 1.93 & 1.90 & 1.88 & 1.81 & 1.76 \\
\hline Poland & 0.67 & 0.68 & 0.70 & 0.66 & 0.64 & 0.56 & 0.54 & 0.56 & 0.57 & 0.56 & 0.57 & 0.60 \\
\hline Portugal & 0.62 & 0.69 & 0.75 & 0.80 & 0.85 & 0.73 & 0.71 & 0.75 & 0.78 & 0.99 & 1.17 & 1.50 \\
\hline $\begin{array}{l}\text { Slovak } \\
\text { Republic }\end{array}$ & 1.09 & 0.79 & 0.66 & 0.65 & 0.64 & 0.57 & 0.57 & 0.51 & 0.51 & 0.49 & 0.46 & 0.47 \\
\hline $\begin{array}{l}\text { Czech } \\
\text { Republic }\end{array}$ & 1.09 & 1.17 & 1.16 & 1.23 & 1.22 & 1.20 & 1.25 & 1.25 & 1.41 & 1.55 & 1.54 & 1.47 \\
\hline $\begin{array}{l}\text { United } \\
\text { Kingdom }\end{array}$ & 1.81 & 1.80 & 1.87 & 1.86 & 1.87 & 1.79 & 1.75 & 1.68 & 1.73 & 1.75 & 1.78 & 1.77 \\
\hline Sweden & 3.54 & .. & 3.65 & .. & 4.29 & .. & 3.80 & 3.58 & 3.56 & 3.68 & 3.40 & 3.70 \\
\hline Switzerland & .. &.. &.. & 2.57 &.. & .. & .. & 2.90 & .. & .. & .. & 3.00 \\
\hline Turkey & 0.49 & 0.50 & 0.63 & 0.64 & 0.72 & 0.53 & 0.48 & 0.52 & 0.59 & 0.58 & 0.72 & 0.73 \\
\hline Total OECD & 2.13 & 2.15 & 2.19 & 2.23 & 2.28 & 2.21 & 2.21 & 2.18 & 2.21 & 2.24 & 2.27 & 2.33 \\
\hline
\end{tabular}

Sources: Institut de la statistique du Québec, Indicateurs de l'activité scientifique et technologique au Québec, Compendium, Éditions 2006 et 2011. For Québec and Canada data: Statistics Canada: Table 358-0001, CANSIM. 
Table 3.E-14: BERD, percentage of GDP, Québec, Canada and OECD countries, 1997-2008

\begin{tabular}{|c|c|c|c|c|c|c|c|c|c|c|c|c|}
\hline & 1997 & 1998 & 1999 & 2000 & 2001 & 2002 & 2003 & 2004 & 2005 & 2006 & 2007 & 2008 \\
\hline Germany & 1.51 & 1.54 & 1.67 & 1.73 & 1.72 & 1.72 & 1.76 & 1.74 & 1.72 & 1.77 & 1.77 & 1.86 \\
\hline Australia & 0.75 & 0.69 & 0.66 & 0.75 & 0.87 & 0.86 & 0.90 & 0.94 & 1.04 & 1.16 & 1.26 & 1.35 \\
\hline Austria & .. & 1.12 &.. & &.. & 1.43 & .. & 1.53 & 1.73 & 1.73 & 1.78 & 1.88 \\
\hline Belgium & 1.34 & 1.35 & 1.40 & 1.45 & 1.54 & 1.37 & 1.31 & 1.28 & 1.25 & 1.29 & 1.32 & 1.32 \\
\hline Canada & 0.99 & 1.06 & 1.06 & 1.15 & 1.29 & 1.17 & 1.16 & 1.17 & 1.14 & 1.14 & 1.09 & 0.99 \\
\hline Québec & 1.34 & 1.41 & 1.44 & 1.62 & 1.79 & 1.72 & 1.66 & 1.65 & 1.53 & 1.71 & 1.67 & 1.52 \\
\hline Korea & 1.80 & 1.65 & 1.61 & 1.77 & 1.97 & 1.80 & 1.89 & 2.06 & 2.15 & 2.32 & 2.45 & 2.53 \\
\hline Denmark & 1.18 & 1.32 & 1.41 & .. & 1.64 & 1.73 & 1.78 & 1.69 & 1.68 & 1.66 & 1.80 & 2.01 \\
\hline Spain & 0.39 & 0.45 & 0.44 & 0.49 & 0.48 & 0.54 & 0.57 & 0.58 & 0.60 & 0.67 & 0.71 & 0.74 \\
\hline United States & 1.88 & 1.94 & 1.98 & 2.05 & 2.00 & 1.83 & 1.81 & 1.76 & 1.80 & 1.86 & 1.92 & 2.02 \\
\hline Finland & 1.78 & 1.92 & 2.19 & 2.40 & 2.41 & 2.35 & 2.43 & 2.42 & 2.46 & 2.48 & 2.51 & 2.76 \\
\hline France & 1.37 & 1.33 & 1.37 & 1.34 & 1.39 & 1.41 & 1.36 & 1.36 & 1.30 & 1.32 & 1.31 & 1.32 \\
\hline Greece & 0.13 & .. & 0.19 & 0.16 & 0.21 & 0.18 & 0.18 & 0.17 & 0.18 & 0.17 & 0.17 & 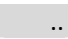 \\
\hline Hungary & 0.30 & 0.26 & 0.28 & 0.35 & 0.38 & 0.35 & 0.34 & 0.36 & 0.41 & 0.48 & 0.49 & 0.52 \\
\hline Ireland & 0.91 & 0.90 & 0.88 & 0.82 & 0.78 & 0.76 & 0.79 & 0.81 & 0.82 & 0.83 & 0.85 & 0.94 \\
\hline Iceland & 0.77 & 0.76 & 1.11 & 1.56 & 1.81 & 1.69 & 1.46 &. & 1.43 & 1.59 & 1.46 & 1.44 \\
\hline Italy & 0.52 & 0.52 & 0.51 & 0.53 & 0.55 & 0.54 & 0.52 & 0.52 & 0.55 & 0.55 & 0.61 & 0.65 \\
\hline Japan & 2.05 & 2.10 & 2.10 & 2.12 & 2.26 & 2.36 & 2.40 & 2.38 & 2.54 & 2.63 & 2.68 & 2.70 \\
\hline Luxembourg & .. & .. & .. & 1.58 & .. & .. & 1.47 & 1.43 & 1.35 & 1.43 & 1.32 & 1.22 \\
\hline Mexico & 0.07 & 0.11 & 0.11 & 0.11 & 0.12 & 0.14 & 0.14 & 0.17 & 0.19 & 0.18 & 0.18 & .. \\
\hline Norway & 0.93 & .. & 0.92 & $\cdot$ & 0.96 & 0.95 & 0.98 & 0.87 & 0.82 & 0.82 & 0.85 & 0.86 \\
\hline New Zealand & 0.31 & .. & 0.30 & .. & 0.42 &.. & 0.48 &.. & 0.47 & .. & 0.50 & .. \\
\hline Netherlands & 1.11 & 1.05 & 1.14 & 1.11 & 1.10 & 0.98 & 1.01 & 1.03 & 1.01 & 1.01 & 0.96 & 0.88 \\
\hline Poland & 0.26 & 0.28 & 0.29 & 0.24 & 0.23 & 0.11 & 0.15 & 0.16 & 0.18 & 0.18 & 0.17 & 0.19 \\
\hline Portugal & 0.14 & 0.16 & 0.17 & 0.22 & 0.27 & 0.24 & 0.24 & 0.27 & 0.30 & 0.46 & 0.60 & 0.75 \\
\hline $\begin{array}{l}\text { Slovak } \\
\text { Republic }\end{array}$ & 0.82 & 0.52 & 0.41 & 0.43 & 0.43 & 0.37 & 0.32 & 0.25 & 0.25 & 0.21 & 0.18 & 0.20 \\
\hline $\begin{array}{l}\text { Czech } \\
\text { Republic }\end{array}$ & 0.69 & 0.75 & 0.73 & 0.74 & 0.74 & 0.73 & 0.76 & 0.78 & 0.89 & 1.01 & 0.95 & 0.91 \\
\hline $\begin{array}{l}\text { United } \\
\text { Kingdom }\end{array}$ & 1.18 & 1.18 & 1.25 & 1.21 & 1.24 & 1.16 & 1.11 & 1.05 & 1.06 & 1.08 & 1.11 & 1.10 \\
\hline Sweden & 2.66 & * & 2.74 & & 3.31 &. & 2.83 & 2.63 & 2.59 & 2.75 & 2.47 & 2.74 \\
\hline Switzerland & .. & .. &. & 1.90 &.. & .. & .. & 2.14 &.. & .. & .. & 2.20 \\
\hline Turkey & 0.16 & 0.16 & 0.24 & 0.21 & 0.24 & 0.15 & 0.11 & 0.13 & 0.20 & 0.21 & 0.30 & 0.32 \\
\hline Total OECD & 1.45 & 1.48 & 1.51 & 1.55 & 1.57 & 1.50 & 1.49 & 1.47 & 1.51 & 1.55 & 1.58 & 1.62 \\
\hline
\end{tabular}

Sources: Institut de la statistique du Québec, Indicateurs de l'activité scientifique et technologique au Québec, Compendium, Éditions 2006 et 2011. For Québec and Canada data: Statistics Canada, Table 358-0001, CANSIM. 
Table 3.E-15: GERD, by performer, Québec, 1981-2008, 2002 constant prices

\begin{tabular}{|c|c|c|c|c|c|c|c|}
\hline & Total & $\begin{array}{r}\text { Federal } \\
\text { government }\end{array}$ & $\begin{array}{r}\text { Government } \\
\text { of Québec }\end{array}$ & $\begin{array}{r}\text { Provincial } \\
\text { research } \\
\text { organizations }\end{array}$ & $\begin{array}{r}\text { Business } \\
\text { enterprise }\end{array}$ & $\begin{array}{r}\text { Higher } \\
\text { education }\end{array}$ & $\begin{array}{r}\text { Private } \\
\text { non- } \\
\text { profit }\end{array}$ \\
\hline 1981 & 1465 & 120 & 36 & 18 & 862 & 429 & 0 \\
\hline 1982 & 1575 & 139 & 38 & 15 & 950 & 432 & 1 \\
\hline 1983 & 1554 & 157 & 33 & 16 & 923 & 425 & 0 \\
\hline 1984 & 1881 & 236 & 43 & 18 & 1108 & 476 & 0 \\
\hline 1985 & 2345 & 274 & 62 & 27 & 1280 & 702 & 0 \\
\hline 1986 & 2366 & 217 & 62 & 21 & 1352 & 714 & 0 \\
\hline 1987 & 2562 & 204 & 62 & 29 & 1516 & 751 & 0 \\
\hline 1988 & 2747 & 202 & 65 & 23 & 1520 & 925 & 12 \\
\hline 1989 & 2922 & 268 & 70 & 25 & 1560 & 987 & 12 \\
\hline 1990 & 3167 & 280 & 68 & 23 & 1717 & 1065 & 14 \\
\hline 1991 & 3395 & 275 & 69 & 22 & 1792 & 1215 & 22 \\
\hline 1992 & 3643 & 292 & 66 & 23 & 1914 & 1340 & 8 \\
\hline 1993 & 3795 & 303 & 62 & 19 & 2067 & 1334 & 10 \\
\hline 1994 & 4023 & 304 & 59 & 18 & 2331 & 1288 & 23 \\
\hline 1995 & 4123 & 275 & 52 & 27 & 2525 & 1232 & 12 \\
\hline 1996 & 4170 & 270 & 52 & 25 & 2613 & 1199 & 11 \\
\hline 1997 & 4264 & 248 & 51 & 12 & 2717 & 1221 & 15 \\
\hline 1998 & 4718 & 278 & 47 & 19 & 2995 & 1379 & 0 \\
\hline 1999 & 5237 & 302 & 44 & 14 & 3244 & 1632 & 1 \\
\hline 2000 & 5846 & 398 & 46 & 13 & 3723 & 1665 & 1 \\
\hline 2001 & 6488 & 418 & 54 & 14 & 4203 & 1798 & 1 \\
\hline 2002 & 6745 & 436 & 65 & 17 & 4153 & 2074 & 0 \\
\hline 2003 & 6743 & 352 & 66 & 14 & 4041 & 2270 & 0 \\
\hline 2004 & 6795 & 345 & 64 & 13 & 4059 & 2314 & 0 \\
\hline 2005 & 6597 & 410 & 68 & 9 & 3788 & 2322 & 0 \\
\hline 2006 & 7002 & 405 & 68 & 7 & 4274 & 2248 & 0 \\
\hline 2007 & 6918 & 352 & 70 & 8 & 4245 & 2243 & 0 \\
\hline 2008 & 6504 & 340 & 72 & 7 & 3785 & 2300 & 0 \\
\hline
\end{tabular}

Source: Statistics Canada. Table 358-0001 - Gross domestic expenditure on research and development, CANSIM. 
Table 3.E-16: R\&D Personnel, Québec, Ontario and Canada, 2002 to 2008

\begin{tabular}{|c|c|c|c|c|c|c|c|}
\hline & 2002 & 2003 & $2004 r$ & $2005 r$ & $2006 r$ & $2007 r$ & $2008 p$ \\
\hline \multicolumn{8}{|l|}{ Québec } \\
\hline Total (FTE) & 57040 & 60710 & 63960 & 64840 & 68970 & 73410 & 75180 \\
\hline Annual variation (\%) & 4.5 & 6.4 & 5.4 & 1.4 & 6.4 & 6.4 & 2.4 \\
\hline Researchers (\%) & 60.7 & 59.7 & 59.1 & 59.7 & 59.5 & 58.6 & 57.6 \\
\hline \multicolumn{8}{|l|}{ Ontario } \\
\hline Total (FTE) & 84500 & 89870 & 95260 & 99330 & 105560 & 111420 & 108650 \\
\hline Annual variation (\%) & 2.0 & 6.4 & 6.0 & 4.3 & 6.3 & 5.6 & -2.5 \\
\hline Researchers (\%) & 66.2 & 64.7 & 63.4 & 64.1 & 62.5 & 62.0 & 63.4 \\
\hline \multicolumn{8}{|l|}{ Canada } \\
\hline Total (FTE) & 183420 & 196510 & 210590 & 218610 & 229160 & 245170 & 242680 \\
\hline Annual variation (\%) & 2.3 & 7.1 & 7.2 & 3.8 & 4.8 & 7.0 & -1.0 \\
\hline Researchers (\%) & 63.3 & 62.7 & 61.9 & 62.6 & 61.4 & 60.9 & 61.4 \\
\hline
\end{tabular}

Source: Institut de la statistique du Québec, Compendium d'indicateurs de l'activité scientifique et technologique au Québec, Édition 2011, Décembre 2011.

Note: $\quad$ : revised; p: preliminary.

Table 3.E-17: R\&D Personnel, by Sector, Canada and selected provinces, 2005

\begin{tabular}{|c|c|c|c|c|c|c|c|c|c|c|}
\hline & \multirow{2}{*}{\multicolumn{2}{|c|}{ Québec }} & \multicolumn{2}{|c|}{ Ontario } & \multicolumn{2}{|c|}{ Alberta } & \multicolumn{2}{|c|}{$\begin{array}{c}\text { Britsh } \\
\text { Columbia }\end{array}$} & \multicolumn{2}{|c|}{ Canada } \\
\hline & & & F1 & & $\mathrm{FT}$ & $\mathrm{E}$ & FT & $\mathrm{EE}$ & FT & \\
\hline & n & $\%$ & n & $\%$ & n & $\%$ & $\mathrm{n}$ & $\%$ & n & $\%$ \\
\hline Total & 65130 & 100.0 & 96290 & 100.0 & 14000 & 100.0 & 21160 & 100.0 & 213930 & 100.0 \\
\hline $\begin{array}{l}\text { Business } \\
\text { entreprise }\end{array}$ & 45210 & 69.4 & 66070 & 68.6 & 6600 & 47.1 & 13640 & 64.5 & 137690 & 64.4 \\
\hline Higher education & 16000 & 24.6 & 21450 & 22.3 & 5860 & 41.9 & 6560 & 31.0 & 56950 & 26.6 \\
\hline $\begin{array}{l}\text { Federal } \\
\text { government }\end{array}$ & 2990 & 4.6 & 8320 & 8.6 & 870 & 6.2 & 770 & 3.6 & 15250 & 7.1 \\
\hline $\begin{array}{l}\text { Provincial } \\
\text { government }\end{array}$ & 930 & 1.4 & 450 & 0.5 & 670 & 4.8 & 190 & 0.9 & 2620 & 1.2 \\
\hline
\end{tabular}

Source: Statistics Canada, no 88-001-X, May 2008, author compilation. 

PART II

EMPIRICAL ANALYSIS 



\section{CHAPTER 4}

\section{Data Sources and Construction of Datasets}




\subsection{Introduction}

An important part of the work undertaken in this research project was to set up the data and build the various datasets used in the Chapters 5-9. For this purpose, several data file sources, consisting of census data and of administrative data were linked. It was a very challenging task, not only because of their complexity, but also because there was no unique identifier among these files. However, that work permitted to build original and information-rich databases. To our knowledge, a work of this magnitude has never been done before on Québec data. This chapter presents the process of matching data and its results. Note that only the results of the success of matching the files are presented in this chapter. Firms' descriptive statistics and their use of government incentives are presented in subsequent chapters since they are related to the subjects addressed in these chapters.

Four main data sources are used in this research project, namely the Québec portion of the following surveys: the annual Survey of Research and Development in Canadian Industry (RDCI), the Survey of Manufactures (ASM) and the Survey of Innovation 2005 (or the IS2005 as we refer to it throughout this document), all from Statistics Canada. The fourth data source consists of administrative data from Revenu Québec (ADRQ). Given the specific needs for the empirical analysis, we constructed three different datasets:

- In Chapter 5 and 6, the dataset used is based on three matched files: the $\mathrm{RDCl}$ survey data, the ASM survey data and the ADRQ. In what follows, we note this dataset RDCI ADRQ-ASM.

- In Chapter 7, the dataset used is based on two matched files: the RDCI survey data and the ADRQ. We note this dataset RDCI-ADRQ.

- $\quad$ Finally, in Chapter 8, the dataset used is based on four matched files: data from IS2005, the RDCl survey data, the ASM survey data and the ADRQ. We note this dataset IS2005-RDCI ADRQ-ASM.

It should be noted that special attention has been given to the information and statistics published throughout this research project due to the fact that most of the data used are prohibited by law from releasing because they comprises nominal information of firms. Under the Federal Statistics Act, Statistics Canada is prohibited from releasing any data which would divulge information that relates to any identifiable person, business or organization without the prior knowledge or the consent in writing of that person, business or organization. The same is also true in the case of Revenu Québec under the provincial Statistics Acts.

Before turning to a formal presentation of the matching process, we first present in the next section a detailed description of the four microdata files used in this research project. 


\subsection{Data Sources}

\subsubsection{Survey of Research and Development in Canadian Industry (RDCI)}

The $\mathrm{RDCl}$ survey is a firm-level survey conducted on an annual basis by Statistics Canada. This survey that started more than 50 years ago collects data to monitor science and technology related activities such as expenditures and personnel devoted annually to R\&D activities by Canadian Industry and non-profit industrial research institutes and associations. The definition of R\&D used by the survey complies with the OECD guidelines as outlined in the Frascati Manual (OECD (2002)). The RDCl survey is a census with cross sectional design and its sampling frame comprises all Canadian firms known or believed to perform or fund R\&D. Since 1997, Statistics Canada has introduced a new methodology for estimating $R \& D$ expenditure ${ }^{11}$. Thus, in order to reduce the reporting burden on firms, only firms performing or funding more than $\$ 1$ million in R\&D are surveyed. For all the other firms, the data are extracted from administrative data from CRA.

Data are collected and reported at the company or Provincial-enterprise ${ }^{12}$ level for this survey. This is so because multi-establishments enterprises often have a centralized unit research.

The survey form permits to collect actual data for the year preceding the survey, temporary data for the survey year and forecasts for the following year. Each year, the survey is held and, as aforementioned, data are collected for all Canadian firms known or believed to perform or fund R\&D and this, from the administrative data of Canada Revenue Agency or directly from survey respondents who receive a survey long-form. For example, in 2000 the survey reported that from a 8396 survey group, more than 7827 firms responded (93\% of the firms). The remaining 569 firms are constituted of inactive, non-localized, out of business or non-responding firms. The 7827 responding firms break into 468 survey long-form and 7359 firms from administrative data of Canada Revenue Agency. Detailed description of the methodology used by the survey can be found in the Industrial Research and Development (Statistics Canada (2002)).

\subsubsection{Annual Survey of Manufactures (ASM)}

The ASM is a survey conducted annually by Statistics Canada for several decades. The purpose of this survey is to collect the financial and commodity information used to compile statistics on Canada's manufacturing industries. Data collected

\footnotetext{
${ }^{11}$ For firms located in provinces other than Québec, the change in the methodology for estimating R\&D expenditures was introduced in 1996.

${ }^{12}$ The notion of "Provincial-enterprise" is used to designate all establishments of a given enterprise within a province and in the same industry as identified by the 4-digit North American Industry Classification System (NAICS) 1997.
} 
permits to compile financial information such as employment, wages, total cost of materials, total sales of manufactured products, inventories, value added by manufacturing and capital expenditures. It also provides other principal industrial statistics such as energy consumption and information on commodities consumed, produced and shipped.

Contrarily to the $\mathrm{RDCl}$ survey described above that collect and report data at the company or enterprise level, the reporting unit in the case of the ASM survey is the establishment. More specifically, the universe of this survey consists of all establishments primarily engaged in manufacturing activities, that is, all establishments classified to sectors 31, 32 and 33 under the NAICS (North American Industry Classification System.

The ASM is one of the largest of all the business surveys conducted by Statistics Canada with data from approximately 100,000 Canadian manufacturers in 2003. Two sources are used to collect data, the data collected directly from firms' respondents by questionnaires and the data obtained from administrative files from Canada Revenue Agency.

Starting from 2004, Statistics Canada implemented major conceptual and methodological changes to the Annual Survey of Manufactures. First, this survey has been amalgamated to the Annual Survey of Forestry to form the Annual Survey of Manufactures and Logging (ASML) and second, Statistics Canada has decided to administer the new designed survey as part of the Unified Enterprise Survey program (UES). The UES program is an annual business survey designed primarily with the goal to integrate into one platform the approximately 200 separate business surveys conducted by Statistics Canada into a single master survey program. In the rest of this document, we will refer to this survey by its historical name, that is to say, the Annual Survey of Manufactures or the ASM that correspond also to the name of the survey during our study period.

\subsubsection{Canadian Survey of Innovation 2005}

The Canadian Survey of Innovation is a survey conducted since 1993 by Statistics Canada every 3-4 year with each survey spanning a 3-year reference period. This survey collects information on innovation and innovation activities of Canadian businesses and their characteristics. The industries surveyed varied from survey to survey, but all the surveys were carried out according to the OECD-wide definitions of the Oslo and Frascatti manuals (OECD (1996), OECD (2002)). The IS2005 which is used in this research project covers the years 2002 to 2004 and targeted a population constituted of all establishments in the manufacturing sector (NAICS 3133) and logging (NAICS 1133) with at least 20 employees and at least \$CAN 250,000 revenues. The questionnaire comprises forty-two questions organized in twelve main sections. For the manufacturing sector, the sample was drawn from Statistics Canada's Business Register from a population of 17726 Canadian manufacturing 
establishments. For Québec, the survey consisted of a census of the target population, which comprises 4622 establishments.

\subsubsection{Administrative Data from Revenu Québec (ADRQ)}

ADRQ comprises essentially fiscal data from R\&D performers claiming tax credits located in Québec or that have R\&D conducted on their behalf in Québec. This is a slight difference compared to the target population of Statistics Canada's RDCI survey described above which does not include firms that contract out their R\&D activities. However, since Statistics Canada and Revenu Québec use both the same definition for qualified research expenditure, the nature of R\&D activities being considered in the RDCl survey is therefore similar to that of Revenu Québec's administrative data.

On the other hand, the reporting unit in the present dataset is the Provincialenterprise which is the same as in the case of the RDCl data survey of Statistics Canada.

The file of administrative data consists of records of the actual amounts fiscal incentives to $R \& D$ effectively received by firms. The main fiscal incentive in this dataset is the refundable tax credit for SR\&ED which comprises the following four components:

- The refundable tax credit for salaries and wages of researchers.

- The refundable tax credit for university research or research carried out by a public research centre or a research consortium.

- The refundable tax credit for pre-competitive research.

- The tax credit for fees or dues paid to a research consortium.

The other fiscal incentives in this dataset include essentially measures that have been withdrawn already, namely the tax credit based on the increase in R\&D (19992004) and the superdeductions for R\&D (1999-2000). Detailed description of these measures as well as of the four components of SR\&ED can be found in Chapter 3.

\subsection{Data Matching Process}

\subsubsection{RDCI-ADRQ Matched Data}

The matching process consisted in linking the data from more than 7500 Québecer's firms that ever appeared in the RDCl survey file over the period 19972004 to the ADRQ.

The main difficulty encountered in this step was the fact that both datasets do not have a unique identifier. While Statistics Canada uses the company number to identify establishments in the RDCI file, Revenu Québec uses a different identifier called NEQ (numéro d'entreprise du Quebéc). However, since the file from Statistics 
Canada includes the names and the addresses of the surveyed firms, it was decided to obtain first the corresponding NEQ from the registry of enterprises from Québec's Inspector General of Financial Institutions (IGIF). The obtained NEQ was then added to the RDCl file and used as linking variable between both files.

Results of data matching in this step are summarized in Table 4.3-1. As can be seen in the fourth column, the linked data exhibit high matching rates over the entire period. This percentage varies from $78.7 \%$ in 1997 to more than $90.0 \%$ in 2003. In other hand, it is interesting to note the increase in the number of firms in the $\mathrm{RDCl}$ data file, i.e. from 3834 firms in 1997 to 6181 in 2003. The implementation of new fiscal measures in Québec aimed at suppoting R\&D such as the tax credit based on the increase of R\&D expenditures or the superdeductions for R\&D by the end of last decade played an important role in the increase in the number of R\&D performers or sub-contracting firms. The R\&D spending doubled in the same period from $\$ 1.8$ billion to $\$ 3.6$ billion.

Table 4.3-1: Summary of matching success in first step and R\&D expenditures in samples

\begin{tabular}{ccccc}
\hline Year & $\begin{array}{c}\text { Surveyed firms } \\
\text { in RDCl }\end{array}$ & $\begin{array}{c}\text { RDCl-ADRQ } \\
\text { matches }\end{array}$ & Matched firms & $\begin{array}{c}\text { Total R\&D in RDCl-ADRQ } \\
\text { matched data }\end{array}$ \\
\cline { 2 - 5 } & $\mathrm{n}$ & $\mathrm{n}$ & $\%$ & billion \$ \\
\hline 1997 & 3834 & 3016 & 78.7 & 1.8 \\
1998 & 3997 & 3245 & 81.2 & 2.0 \\
1999 & 4142 & 3645 & 88.0 & 2.6 \\
2000 & 4575 & 4141 & 90.5 & 3.0 \\
2001 & 5077 & 4608 & 90.8 & 3.6 \\
2002 & 5646 & 5093 & 90.2 & 3.6 \\
2003 & 6181 & 5600 & 90.6 & 3.6 \\
\hline
\end{tabular}

Sources: Minstry of Revenue of Québec, Statistics Canada, RDCI survey Compilations: ISQ and the author.

\subsubsection{RDCI-ADRQ-ASM Matched Data}

In this step, the RDCl-ADRQ data set obtained in the preceding matching process is matched to the data from ASM. We used the NEQ as an identifier for linking both files and this, by undertaking the same process as in the previous step: obtain first the NEQ corresponding to each company name in the data file from the registry of enterprises from IGIF. One of the difficulties encountered in doing so was the fact that the company names in the data file were not always written exactly in the same way from year to year. This can be attributed to several facts such as a change in the name of the company, the name of the company being written either in 
French or in English or, more often, this can be simply attributed to writing errors. Any of these cases implied a time consuming process, including search in other variables such as establishment addresses in order to validate comparisons of company names in the ASM data file and in the IGIF data file.

Another difficulty encountered in this step of the matching process was the fact that the aggregating level of both files is different as mentioned earlier. While the RDCI-ADRQ matched data file reports data at the enterprise level, the ASM data file reports data for every establishment. To solve this problem, we grouped the establishments in the ASM data file by enterprise prior to performing the matching in the cases of multi-establishments enterprises. However, not all multiestablishments enterprises were grouped. Hence, the original sample included several cases of merger and acquisition that usually brings important changes in the structure of enterprises. It was decided to discard all of these cases.

The results of the matching between the RDCI-ADRQ data and the ASM survey data are presented in Table 4.3-2. The second column shows the number of manufacturing firms in the RDCI-ADRQ data file. For reference, we indicated in parentheses the total number of firms in RDCI-ADRQ data files as obtained in the previous step, in other words, the total number of firms including all sectors. The third column shows the result of matching between the three datasets and the fourth column summarizes the matching rates. As it can be noticed, these matching rates are high, especially in the period 2000-2003 with rates above 93\%. In the period 1997-1999, even if these rates are still high, they surprisingly decrease from $73.3 \%$ to $69.2 \%$. The gap in matching rates between both periods can be attributed to changes introduced by Statistics Canada in the RDCl survey methodology (see Section 4.2.1).

The fifth column reports the total R\&D expenditures in matched samples. The decrease in R\&D in the 1997-1999 sub-period is more likely to be the result of the decrease in matching rates over that period. The last column shows R\&D expenditures for firms whose ratio of R\&D expenditures to value added is less than one. We computed this ratio as an additional check to test the validity of matched units. It is well known that performing matching from data files collected at different levels may result in a certain percentage of invalid matched units. This issue is likely to occur in our case because the reporting unit is the company or enterprise in the case of both RDCl-ADRQ matched data, but differs from that of the ASM data which collect data at the establishment level. In its Industrial Research and Development (Statistics Canada (2002), pp 41), Statistics Canada notice that comparisons between R\&D data collected at the company level and other data collected at the establishment level, such as "census value added", may be misleading sometimes.

Computing and checking if the ratio of R\&D expenditures to value added is less than one provides a good indication on the validity of matched units. In our case, 
only $2.13 \%$ of the matched units present a ratio of $R \& D$ expenditures to value added above unity which is a very good result. As can be seen, columns 5 and 6 in Table 4.3-2 show no significant differences. It should be noted also that for cases with a ratio of $R \& D$ expenditures to value added above unity, one cannot say at once whether it is a valid match or not. As argued by Rogers (2006)(page 9) confronted with the same issue, these cases may be valid because a firm could buy in $R \& D$, have a low value added, and hence a high ratio of $R \& D$ expenditures to value added.

Table 4.3-2: $\quad$ Summary of matching success in the second step and R\&D expenditures in samples

\begin{tabular}{|c|c|c|c|c|c|}
\hline \multirow[t]{2}{*}{ Year } & $\begin{array}{l}\text { Manufactur } \\
\text { ing firms in } \\
\text { RDCl-ADRQ } \\
\text { (all firms) }\end{array}$ & $\begin{array}{l}\text { RDCl-ADRQ- } \\
\text { ASM matches }\end{array}$ & $\begin{array}{l}\text { Matched } \\
\text { firms }\end{array}$ & $\begin{array}{l}\text { Total R\&D in RDCl- } \\
\text { ADRQ matched data }\end{array}$ & $\begin{array}{l}\text { Total R\&D (if } \\
\text { R\&D/VA }<1 \text { ) }\end{array}$ \\
\hline & $\mathrm{n}$ & $\mathrm{n}$ & $\%$ & billions \$ & billions \$ \\
\hline 1997 & $\begin{array}{c}1521 \\
(3016)\end{array}$ & 1175 & $77.3 \%$ & 1.1 & 1.1 \\
\hline 1998 & $\begin{array}{c}1661 \\
(3245)\end{array}$ & 1266 & $76.2 \%$ & 0.9 & 0.8 \\
\hline 1999 & $\begin{array}{c}1885 \\
(3645)\end{array}$ & 1304 & $69.2 \%$ & 0.8 & 0.8 \\
\hline 2000 & $\begin{array}{c}2055 \\
(4141)\end{array}$ & 1916 & $93.2 \%$ & 1.4 & 1.0 \\
\hline 2001 & $\begin{array}{c}2222 \\
(4608)\end{array}$ & 2094 & $94.2 \%$ & 1.0 & 0.9 \\
\hline 2002 & $\begin{array}{c}2501 \\
(5093)\end{array}$ & 2401 & $96.0 \%$ & 1.0 & 0.9 \\
\hline 2003 & $\begin{array}{c}2746 \\
(5600)\end{array}$ & 2664 & $97.0 \%$ & 0.9 & 0.8 \\
\hline
\end{tabular}

Sources: $\quad$ Minstry of Revenue of Québec, Statistics Canada, RDCl survey, ASM survey. Compilation: ISQ and the author.

Note: $\quad$ The numbers in parentheses represents the total numbers of firms in samples including other sectors.

\subsubsection{IS2005-ASM-RDCI-ADRQ Matched Data}

For the purposes of the estimations in Chapter 8, two datasets are constructed from the four data sources presented above: the IS2005-ASM dataset and the IS2005ASM-RCDI-ADRQ dataset.

For the construction of the IS2005-ASM dataset, we matched the Québec portion of the Survey of Innovation 2005 and of the Annual Survey of Manufactures and Logging, years 2002 and 2004. In both surveys, the reporting unit is the establishment and the pairing was done using Statistics Canada's establishment 
statistical number. From the 3161 observations $^{13}$ in the initial database, only the ones matching with both years of the Annual Survey of Manufactures and Logging (2002 and 2004) were kept and the sample was standardized to target the same population as the one in the OECD countries. The Québec final sample comprised 2747 observations.

For the construction of the IS2005-ASM-RCDI-ADRQ dataset, we used the matched dataset obtained previously and linked it to the RDCl and the ADRQ data for the years 2002 to 2004 . Pairing the matched establishment database to the two databases on $R \& D$ required some manipulations since the reporting unit in the RDCI survey as well as in the ADRQ files is the enterprise rather than the establishment. The goal of the pairing was to assign the enterprise information on R\&D to the correct establishment in the matched database. Since it is not possible to disaggregate enterprise information between their establishments and thus to correctly assign the R\&D information to the establishments, all the multiestablishments firms were withdrawn from the matched database. To do so, we used the Statistics Canada's Business Register (June 2005 version), the frame of the Survey of Innovation 2005, to identify multi-establishment firms. The target population was therefore changed to include only single establishment firms ${ }^{14}$. The new target population of the matched database is single establishment firms in the manufacturing sector (NAICS 31-33) with at least 20 employees and at least $\$ 250,000$ revenues.

The pairing of the $\mathrm{RDCl}$ and the matched single establishment database was done using Statistics Canada's enterprise statistical number. There is no common identification variable between the three surveys and the Revenu Québec administrative files. The pairing was therefore made with the legal name and address of the enterprise using a SAS macro designed for this purpose. For all the remaining unpaired observations, the NEQ was sought in the Québec Enterprise Register. A NEQ was assigned to an observation when there was a concordance of the enterprise name and address. At the end of the matching process and cleaning, we obtained a sample with 2166 observations.

\footnotetext{
${ }^{13}$ The initial database comprises 3161 observations, which represented a weighted population of 4310 establishments.

${ }^{14}$ It should be noted that the definition of a single establishment in Québec firms does not exclude companies with locations in Canada or elsewhere in the world where they have only one establishment in Québec.
} 



\section{CHAPTER 5}

\section{Effectiveness of R\&D Tax Incentives in Manufacturing Sector in Québec}

Rufin Baghana

Pierre Mohnen*

*Maastricht University, UNU-MERIT and CIRANO

Published as: Baghana, Rufin, and Pierre Mohnen, 2009, Effectiveness of R\&D tax incentives in small and large enterprises in Québec, Small Business Economics 33, 91-107. 


\subsection{Introduction}

Many countries rely on a policy of $R \& D$ tax incentives to spur $R \& D$ in the private sector. In some countries, the budget allocated to this policy is substantive. Canada is one of these countries. In 2006, the Canadian federal government spent about $\$ 3$ billion a year on its R\&D tax incentives program (Department of Finance Canada (2007)). Among the Canadian provinces, has been the most generous in its R\&D tax incentives for many years. In 2005, provincial payments for fiscal assistance amounted to $\$ 538$ million dollars. Tax incentives have the virtue of being more neutral in the type of firms and projects funded than measures of direct R\&D support in the form of grants and subsidies, letting private business determine the projects and the amount of R\&D. This virtue could also be seen as a weakness, in so far as it might be socially preferable to steer R\&D towards projects with high spillovers. Many countries try to focus their support on small and medium size enterprises, which are, more than big firms, plagued by the market failure of financing intangible investment in the presence of information asymmetries. As firms rely more on internal funds than on external debt and equity to finance their investments, especially R\&D investments, small firms might be more financially constrained than large firms ${ }^{15}$.

A number of studies have been done to evaluate the effectiveness of tax incentives in making firms spend more on R\&D. The usual evaluation consists in checking whether there is R\&D additionality, in the sense that private firms increase their R\&D expenditures by more than it costs the government to support the tax incentives program. If per dollar of government support less than one dollar of additional private R\&D gets spent by business, then public support partially crowds out private funding for R\&D. The evaluation of the so-called "bang for the buck" is a rough cost-benefit analysis which generally does not take into account factors like spillovers, indirect tax returns, administration costs, and the opportunity cost of spending taxable income on R\&D support.

In the literature there are two approaches to the evaluation of the effectiveness of R\&D tax incentives. The first approach estimates the treatment effect by constructing counterfactuals, instrumenting the treatment, comparing experimental and treated firms before and after the introduction of a policy change, or by comparing firms that are close to a discontinuity in the treatment design. Matching estimators compare the average $R \& D$ effort of firms that receive $R \& D$ tax credits with the average R\&D of firms that do not, but that are otherwise similar, in

\footnotetext{
${ }^{15}$ See the literature on the cash-flow sensitivity of investment following the seminal work of Fazzari, Hubbard and Petersen (1988).
} 
particular, in having the same likelihood of receiving R\&D tax credits (Czarnitzki, Hanel and Rosa (2005), Corchuelo Martínez-Azúa and Martínez-Ros (2008), Duguet (2008)). Instrumental variables estimators instrument the treatment by regressing it on a certain number of explanatory variables (Cappelen, Raknerud and Rybalka (2008)) or by controlling for the selected firms that know about R\&D tax incentives and apply for it (Corchuelo Martínez-Azúa and Martínez-Ros (2008)). Difference-indifferences estimators compare the R\&D of firms in the reference and treated group before and after a policy change, in this case a new feature in R\&D tax incentives (Cornet and Vroomen (2005)). Regression discontinuity design compares the R\&D of firms that are affected or unaffected by an exogenous discontinuity in the treatment function, for example just below and just above a ceiling in the conditions for being eligible to receive R\&D tax credits (Hægeland and Møen (2007)).

The second approach is based on a structural model deriving from some kind of optimization objective a demand for R\&D equation that depends inter alia on a user cost of R\&D itself a function of R\&D tax credit parameters (Bernstein (1986); Hall (1993); Bloom, Griffith and Van Reenen (2002); Mairesse and Mulkay (2004)). The structural modeling approach permits simulations of the effects of future tax changes and allows distinguishing between short-run and long-run effects. Given the endogeneity of the amount of R\&D tax credits received and a possible distributed lag specification, instrumental variable estimations are used.

This chapter evaluates the effectiveness of the R\&D tax incentives program in the province of Québec. We rely on a modeling of factor demand similar to the ones adopted in studies that have opted for an estimation of a factor demand model for $R \& D$ with $R \& D$ tax parameters included in the user cost of R\&D (as in Bloom et al. (2002), Mairesse and Mulkay (2004), Dagenais, Mohnen and Therrien (2004), Hægeland and Møen (2007)). We construct an observational specific B-index that enters the user cost of capital and that reflects the various changes over time and across firms in the R\&D tax credit scheme in Québec and in Canada. As opposed to most other studies, we have access to administrative data capturing the real R\&D tax support received by firms operating in the province of Québec. For a survey of the empirical literature on R\&D tax incentives, see Hall and Van Reenen (2000) and Mohnen (2000). For previous work on Canadian firms, see Bernstein (1986), Lebeau (1996), Department of Finance and Revenue Canada (1997), Dagenais et al. (2004), Czarnitzki et al. (2005), and Parsons and Phillips (2007).

The originality of our empirical analysis is that it exploits the actual R\&D tax credits received by every firm instead of just relying on the statutory tax rates and eligibility conditions and attributing tax incentives to every eligible firm. Indeed, many firms may either not know of the existence of tax incentives (especially small firms) or decide not to apply for R\&D tax credits because of administration costs, inexperience or apprehension in dealing with the tax authorities. The second 
advantage of using the observed payments is that it does not oblige us to collect data on certain types of R\&D, like cooperative R\&D, that would be difficult to get. The third advantage has to do with the fact that in the R\&D surveys, firms operating in more than one province may not always split their R\&D by province, whereas from the administrative data, the provincial figures are declared automatically. Few studies in the literature have been able to make use of the actual payments of tax incentives (Brouwer, Den Hertog, Poot and Segers (2002); Hægeland and Møen (2007); De Jong and Verhoeven (2007); Duguet (2008)).

Since the tax support is more generous towards small and medium sized enterprises, it is of interest to compare the effectiveness of this policy for SMEs and large enterprises. Lokshin and Mohnen (2007) report that small firms (with less than 200 employees) are more sensitive to R\&D tax incentives than large firms in the Netherlands. Small firms are likely to be more reactive to changes in R\&D tax incentives (at least if they actually apply for them) as they have more difficulty in financing their R\&D. They have little collateral, they may be young firms with little to show in terms of success, they may not even have patents to signal their capability to innovate. The estimation of the model and the evaluation of the program in terms of R\&D additionality will be done separately for both types of firms. We want to investigate whether the same holds for firms in Québec.

The rest of this chapter is organized as follows. Section 5.2 briefly presents the data and, hence, provides an overview of the characteristics of manufacturing firms in Québec. Section 5.3 sketches the structural model of demand for R\&D from which we shall estimate the price elasticity of R\&D. Section 5.4 presents and comments on the estimation results. Section 5.5 presents some simulation analyses of R\&D tax experiments to calculate the ratio of additional R\&D per dollar of tax expenditure for different modules of the R\&D tax credit and to show differences in the effectiveness of R\&D tax incentives for small and large firms. Section 5.6 concludes.

\subsection{Data and Firms Characteristics}

Data used in this chapter is the result of the matching of three different microdata files. The first two are survey files from Statistics Canada: The RDCI Survey and the ASM Survey. The third file comes from administrative data of Revenu Québec and is constituted of fiscal data, more precisely tax credits and other fiscal incentives for the firms performing R\&D activities. Matching was performed at the level of 
Provincial-enterprises ${ }^{16}$ and the three files contained only firms located in Québec (see Chapter 4 for more details on the data and the matching process).

Combining these three data files was necessary because of their additional nature. For instance, given the fact that $R \& D$ grant recipient firms usually claim also $R \& D$ tax credits, it is of great interest for analysis needs to gather information on the amounts of both types of public support allocated annually to each firm. To do so in our case, the administrative data file from Revenu Québec had to be matched to the RDCl data file from Statistics Canada which includes R\&D grants, contracts and capital venture, but tax credit data. Furthermore, in order to obtain financial data required for the productivity analysis, it has been decided to match these two microdata files to a third one, namely the ASM survey data, to complete the database. However, this advantage has its drawbacks: the larger part of the dataset is left apart because manufacturing firms performing R\&D are only a little part of the entire universe of R\&D performers.

Breaking R\&D performers by size class in the manufacturing sector between 1997 and 2003, data show that most of the R\&D applications come from small firms, but that the large firms get the greater part of the amount of attributed tax credits. As shown in Table 5.2-1, more than $63.7 \%$ of all tax claimants are small firms ( 0 to 50 employees) making up $28.5 \%$ of the total value of attributed tax credits. However, large firms that represent only $3.9 \%$ of tax claimants get more than $37.5 \%$ of the total amount of attributed tax credits. The tax credit for salaries of researchers was the most important program accounting for at least $75 \%$ of total attributed tax credits. The tax credit for university research shows no significant differences across size classes. Tax credit for pre-competitive research was used more often by small and medium sized firms (less than 500 employees). The reason is that because of limited capacity of funding a research project, firms enter into partnership contracts for pre-competitive research projects (or to have the project done on their behalf). This tax credit and the credit for university research, however, represented only $0.9 \%$ and $0.6 \%$, respectively, of the total attributed tax credit over the sample period. Concerning the tax credit for dues or contributions paid by corporations to research consortium, data show that the majority of beneficiaries were firms with more than 500 employees.

Table 5.2-1 also shows the non-negligible use of other fiscal incentives such as the superdeductions for R\&D and the tax credit based on the increase in R\&D expenditures (see Section 3.3 in Chapter 3 for a detailed description of these fiscal measures). Even though these fiscal measures lasted only 5 and 1 years respectively, claims totaled $4.5 \%$ and $4.0 \%$, respectively, of the total attributed tax credits over the sample period, showing greater popularity of these tax compared

\footnotetext{
${ }^{16}$ The data file from Revenu Québec and RDCl survey from Statistics Canada reports data at the provincial-enterprise level whereas the ASM data files reports data for every establishment. All of these have been grouped in provincial-enterprises prior to performing the matching process.
} 
to three of the SR\&ED tax credits, i.e. the tax credit for university research, the tax credit for pre-competitive research and the tax credit for research consortium.

Finally, turning to the distribution of the tax credit by group in the manufacturing sector, it can be seen in Table 5.2-2 that more than $60 \%$ of all tax claimants are in the medium-low and low technology group with $32.8 \%$ of the total value of attributed tax credits. However, the high technology group which represents only $10 \%$ of tax claimants get more than $42.6 \%$ of the total amount of attributed tax credits. Clearly, these differences suggest lower cost or higher benefits from using tax credits in the high technology group which is R\&D-intensive. The distribution by type of tax credits shows once again the popularity of the tax credit for salaries of researchers with more than $80 \%$ of tax claims across all industry groups in the manufacturing sector. Not surprisingly, the demand for the tax credit for university research was made exclusively by firms in the medium-high and in the medium-low and low technology groups. Firms in these groups tend to subcontract or enter research consortium because they do not necessarily possess their own research department contrary to most firms in the high technology group. In the same vein, it can be stated that, the tax credit for pre-competitive research has been more popular in the high-technology group whereas the tax credit for research consortium has been more popular in the medium-low and low technology group. Table 5.2-2 also reveals the popularity of the tax credit for large firms and the superdeductions for R\&D in the high-technology group and that of the tax credit based on the increase in R\&D expenditures in both the medium-high technology and the medium-low and low technology groups. 


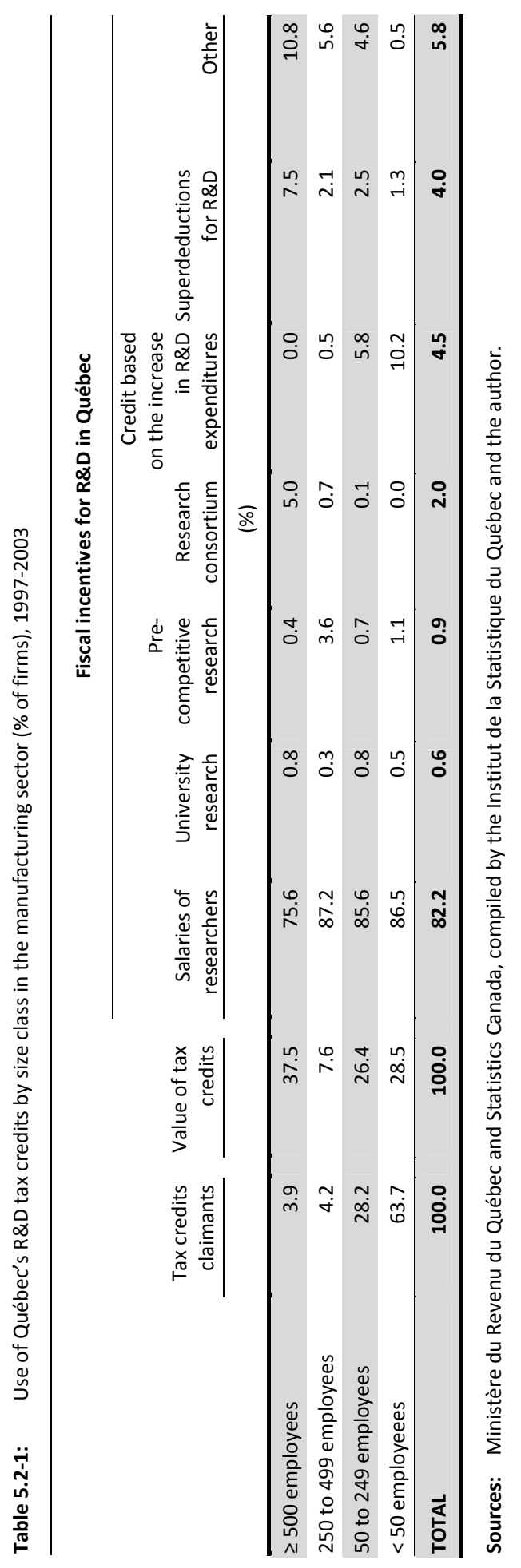




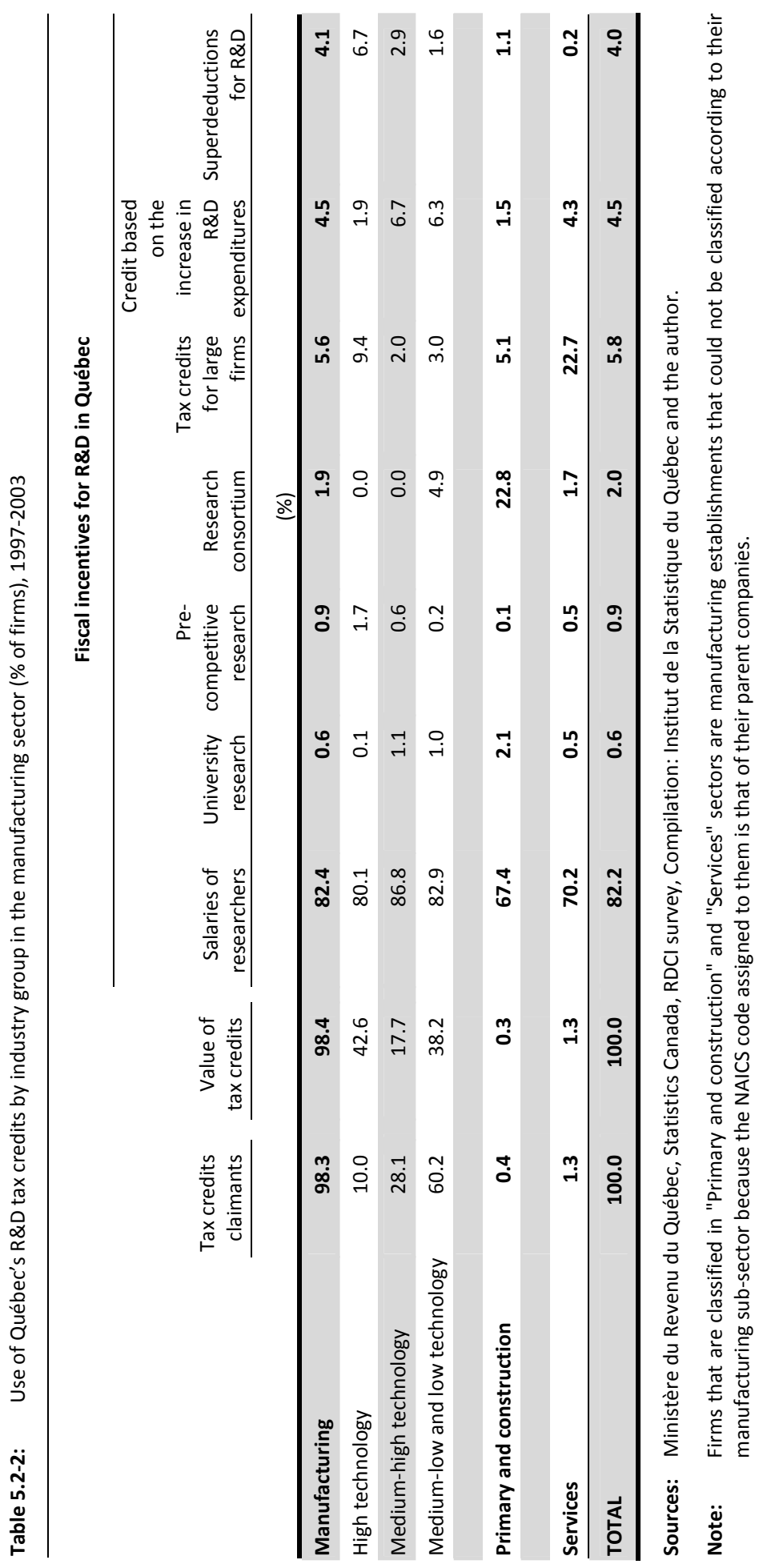




\subsection{The Model}

The model from which we estimate the elasticity of $R \& D$ with respect to its user cost is the same as the one used in Lokshin and Mohnen (2007). In a nutshell, we start from a CES approximation to the true production function for firm $i$ at time $t$ (as in Chirinko, Fazzari and Meyer (1999); Hall and Van Reenen (2000); Mairesse and Mulkay (2004)):

$$
Q_{i t}=F_{t}\left(K_{i t}, X_{i t}\right)=\gamma\left[\beta K_{i t}^{-\rho}+(1-\beta) X_{i t}^{-\rho}\right]^{-v / \rho}
$$

where $Q_{i t}$ is the output, $K_{i t}$ is the end of period R\&D stock, $X_{i t}$ is the other inputs, and $\gamma$ (a scale factor), $\beta$ (the distribution parameter), $v$ (a measure of the returns to scale) are parameters to be estimated that characterize the technology, as well as $\rho$ that enters the expression for the elasticity of substitution $(\sigma)$ between R\&D stock and the other inputs and is given by $\sigma=1 /(1+\rho) \geq 0$ In a static model of profit maximization, the optimal amount of R\&D capital in logarithms would be given by ${ }^{17}$

$k_{i t}^{*}=a+(\sigma+(1-\sigma) / v) q_{i t}-\sigma\left(u_{R, i t}-p_{Q, i t}\right)$

where $u_{R, i t}$ is the logarithm of the user cost of $R \& D$ and $p_{Q, i t}$ the unobservable output price. Following Klette and Griliches (1996) we assume a price elasticity of $\varepsilon>0$ in absolute value of the demand for the output of firm $i$ relative to industry demand:

$q_{i t}-q_{l t}=-\varepsilon\left(p_{Q, i t}-p_{l t}\right)$

where $q_{\mid t}$ is the industry demand and $p_{\mid t}$ the industry price in period $t$. Substituting ( 5-3) into ( 5-2 ) yields the long-run relationship:

$k_{i t}^{*}=a+\varphi v_{i t}-\sigma\left(u_{R, i t}-p_{l, i t}\right)+\gamma q_{l, t}$

where $\varphi=\sigma+\mu(1-\sigma) / v$, and $\gamma=(1-\mu)(1-\sigma) / v$, and $v_{i t}$ is the nominal output deflated by the industry output price deflator.

\footnotetext{
${ }^{17}$ Logarithmically-transformed variables are denoted by small letters.
} 
Investment is composed of a replacement investment $\left(R_{i t}^{r}\right)$ and a net investment $\left(R_{i t}^{n}\right)$. The former is proportional to the R\&D stock at the beginning of the period: $R_{i t}^{r}=\delta K_{i, t-1}$. The latter represents the change in the R\&D stock: $R_{i t}^{n}=K_{i t}-K_{i, t-1}$. Hence we can write:

$\frac{R_{i t}}{K_{i, t-1}}=\frac{R_{i t}^{r}+R_{i t}^{n}}{K_{i, t-1}}=\delta+\frac{\Delta K_{i t}}{K_{i, t-1}}$

We approximate the discrete growth rate in the R\&D stock by a log difference and assume that the growth rate in the $R \& D$ stock follows a partial adjustment mechanism

$k_{t}-k_{t-1}=\lambda\left(k_{t}^{*}-k_{t-1}\right)$

which after substitutions can be rewritten as:

$k_{t}-k_{t-1}=\lambda\left(k_{t}^{*}-k_{t-1}^{*}\right)+(1-\lambda) \lambda\left(k_{t-1}^{*}-k_{t-2}^{*}\right)+(1-\lambda)^{2} \lambda\left(k_{t-2}^{*}-k_{t-3}^{*}\right)+\ldots$

Changes in the R\&D stock are therefore expressed as a weighted sum of changes in the desired R\&D stocks in the past. We can now rewrite ( 5-5) as:

$\frac{R_{i t}}{K_{i, t-1}}=\delta+\varphi \frac{\lambda d v_{i t}}{I-(1-\lambda) L}-\sigma \frac{\lambda\left(d u_{R i t}-d p_{\text {lit }}\right)}{I-(1-\lambda) L}+\gamma \frac{\lambda d q_{\text {lit }}}{I-(1-\lambda) L}+\varepsilon_{i t}$

or as:

$\frac{R_{i t}}{K_{i, t-1}}=\lambda \delta+(1-\lambda) \frac{R_{i, t-1}}{K_{i, t-2}}+\varphi \lambda d v_{i t}-\sigma \lambda\left(d u_{R i t}-d p_{l i t}\right)+\gamma \lambda d q_{l i t}+\left(\varepsilon_{i t}-(1-\lambda) \varepsilon_{i, t-1}\right)$

We have appended a random error term in (5-9) to account for random unobserved disturbances. Any individual effect present in (5-4) is removed by the first-differencing. The error term follows a MA(1) process. Because of the simultaneity between the user cost and the amount of R\&D we have to instrument for the contemporaneous change in the user cost of R\&D. The short-run elasticity of $R \& D$ stock with respect to the user cost of $R \& D$ is given by $-\sigma \lambda$. The long-run elasticity is given by $-\sigma$. 


\subsection{Estimation Results}

Table 5.4-1 gives the magnitude of the variables appearing in the final estimating Equation (5-9).

Table 5.4-1: $\quad$ Variable constructions and descriptive statistics

\begin{tabular}{|c|c|c|c|c|}
\hline Variable & Construction & All firms & $\begin{array}{l}\text { Large } \\
\text { firms }\end{array}$ & Small firms \\
\hline$R / K$ & $\begin{array}{l}\text { Ratio of R\&D flow to R\&D stock, constructed } \\
\text { as own R\&D expenditures divided by } R \& D \\
\text { stock at the beginning of the period }\end{array}$ & $\begin{array}{c}0.24 \\
(0.25)\end{array}$ & $\begin{array}{c}0.25 \\
(0.25)\end{array}$ & $\begin{array}{c}0.23 \\
(0.23)\end{array}$ \\
\hline$u$ & $\begin{array}{l}\text { User cost of R\&D deflated by the industry } \\
\text { output deflator }\end{array}$ & $\begin{array}{c}0.08 \\
(0.05)\end{array}$ & $\begin{array}{c}0.07 \\
(0.05)\end{array}$ & $\begin{array}{c}0.11 \\
(0.03)\end{array}$ \\
\hline$v$ & Firm sales in real terms, in logarithm & $\begin{array}{l}15.31 \\
(1.73)\end{array}$ & $\begin{array}{l}14.85 \\
(1.42)\end{array}$ & $\begin{array}{l}17.64 \\
(1.17)\end{array}$ \\
\hline$q_{l}$ & $\begin{array}{l}\text { Industry sales } \\
\text { (at 4-digit NAICS classification), in logarithm }\end{array}$ & $\begin{array}{l}21.65 \\
(0.64)\end{array}$ & $\begin{array}{l}21.65 \\
(0.63)\end{array}$ & $\begin{array}{l}21.67 \\
(0.65)\end{array}$ \\
\hline$\Delta u$ & $\begin{array}{l}\text { Log-difference in user cost of R\&D deflated } \\
\text { by industry output deflator }\end{array}$ & $\begin{array}{l}0.24 \\
(0.26)\end{array}$ & $\begin{array}{c}0.26 \\
(0.27)\end{array}$ & $\begin{array}{c}0.13 \\
(0.22)\end{array}$ \\
\hline$\Delta v$ & Log-difference in firms' real sales & $\begin{array}{c}0.08 \\
(0.52)\end{array}$ & $\begin{array}{c}0.09 \\
(0.50)\end{array}$ & $\begin{array}{c}0.03 \\
(0.58)\end{array}$ \\
\hline$\Delta q_{1}$ & Log-difference in industry real sales & $\begin{array}{c}0.03 \\
(0.19)\end{array}$ & $\begin{array}{c}0.03 \\
(0.19)\end{array}$ & $\begin{array}{c}0.02 \\
(0.18)\end{array}$ \\
\hline$K$ & R\&D stock, in million 2002 CAN\$ & $\begin{array}{c}4.36 \\
(39.50)\end{array}$ & $\begin{array}{c}10.34 \\
(22.64)\end{array}$ & $\begin{array}{c}21.20 \\
(95.30)\end{array}$ \\
\hline
\end{tabular}

Note: $\quad$ The descriptive statistics are sample means for the years 1997-2003. The base year is 2002.

The R\&D stock has been constructed by the perpetual inventory method, the starting value being obtained by dividing the initial R\&D flow by the sum of the R\&D depreciation rate (taken to be 15\%) and the growth rate of R\&D flow during the sample period. The R\&D stock is on average 5 times bigger than the R\&D flow. All nominal values are converted in 2002 prices by the appropriate deflators. The R\&D deflator is computed as the average of the GDP deflator and the monthly wage index of the manufacturing sector in Québec (Statistics Canada, Cansim, Table 281-0039). The industry price and quantity used to eliminate the firm output in Equation (5-3) is from the NAICS 3-digit industry in which the firm has its major activity.

In Table 5.4-2 we present the GMM estimates for all firms in our sample, and separately for the large firms (with more than 250 employees) and the small firms (with less than 250 employees). As we can see, most of the firms in our sample are small, benefiting from favourable R\&D tax credits. We have relatively few large firms in our sample. Two explanatory variables have to be instrumented: the user 
cost of R\&D, which may vary with the amount of R\&D, and the lagged dependent variable, which is correlated with the $M A(1)$ error term. Without instrumenting, i.e. estimating Equation (5-9) by ordinary least squares, yields a short-run price elasticity $-\sigma \lambda$ of -0.01 (non-significant). We then turn to the GMM estimations. As instrumental variables we use the one-period lagged level of the user cost of R\&D (in log), the two-period lagged level of the dependent variable, and the contemporaneous R\&D deflator, interest rate and tax credit on researchers' salaries in Québec.

The rank test of underidentification rejects the null hypothesis that the matrix of reduced form coefficients has less than full rank, and hence points to the relevance of the instruments and to the identification of the model with those instruments. Regarding the weakness of the instruments, the F-test based on Shea's partial R2 is greater than 10 for the first-stage significance of the exogenous variables in explaining the user cost of $R \& D$, but less than 10 in explaining the lagged dependent variable. However, in the presence of two endogenous variables we should rather use the Cragg Donald statistic (the minimum eigenvalue of the first stage F-statistic matrix) and the Stock and Yogo (2005) critical values for testing whether the instruments are weak (1) in terms of relative bias, i.e. whether the maximum relative squared bias of the IV estimator relative to the OLS estimator is at least some value $b$, and (2) in terms of bias in the Wald test size, i.e. whether the actual size of the test is at least some value $b$ above the nominal level of the test. At the $5 \%$ level, we reject the null hypothesis that the instruments bias the IV estimate relative to the OLS estimate by more than $30 \%$ and we do not even reject the null that the bias in the size of the Wald test exceeds $30 \%$. With our instruments the Sargan test of overidentifying restrictions is accepted. We have explored with different additional instruments (the lagged value of sales in logarithms, the provincial incremental tax credit rate, the ratio of log assets over log employees, and the ratio of growth in output over growth in employment). In each case the test of overidentifying restrictions was rejected. We thus conclude that our instruments are rather weak, but that at least they fulfill the overidentifying restrictions.

The speed of adjustment in the desired $R \& D$ stock $\lambda$ is equal to 0.74 for small firms and 0.57 for large firms, although statistically speaking they are not significantly different from each other. The short-run price elasticity of R\&D is -0.14 for small firms and not significantly different from zero for large firms. It may be that large firms are not very price responsive, but it may also be that our sample size is too small for large firms to yield significant coefficients. For the whole sample, however, the short-run price elasticity is significantly different from zero and lower than for the subsample of small firms. Small firms do hence seem to be somewhat more price responsive than large firms. The long-run price elasticity $\sigma$ is equal to -0.19 for small firms and -0.14 for the whole sample. The long-run output price elasticity is equal to 0.03 for small firms and 0.07 for large firms. From the 
constant term in the regression we can recover the estimated $R \& D$ depreciation rate, which turns out to be between $17 \%$ and $27 \%$, thus not far away from our ad hoc choice of $15 \%$.

Table 5.4-2: Estimation results of R\&D equation

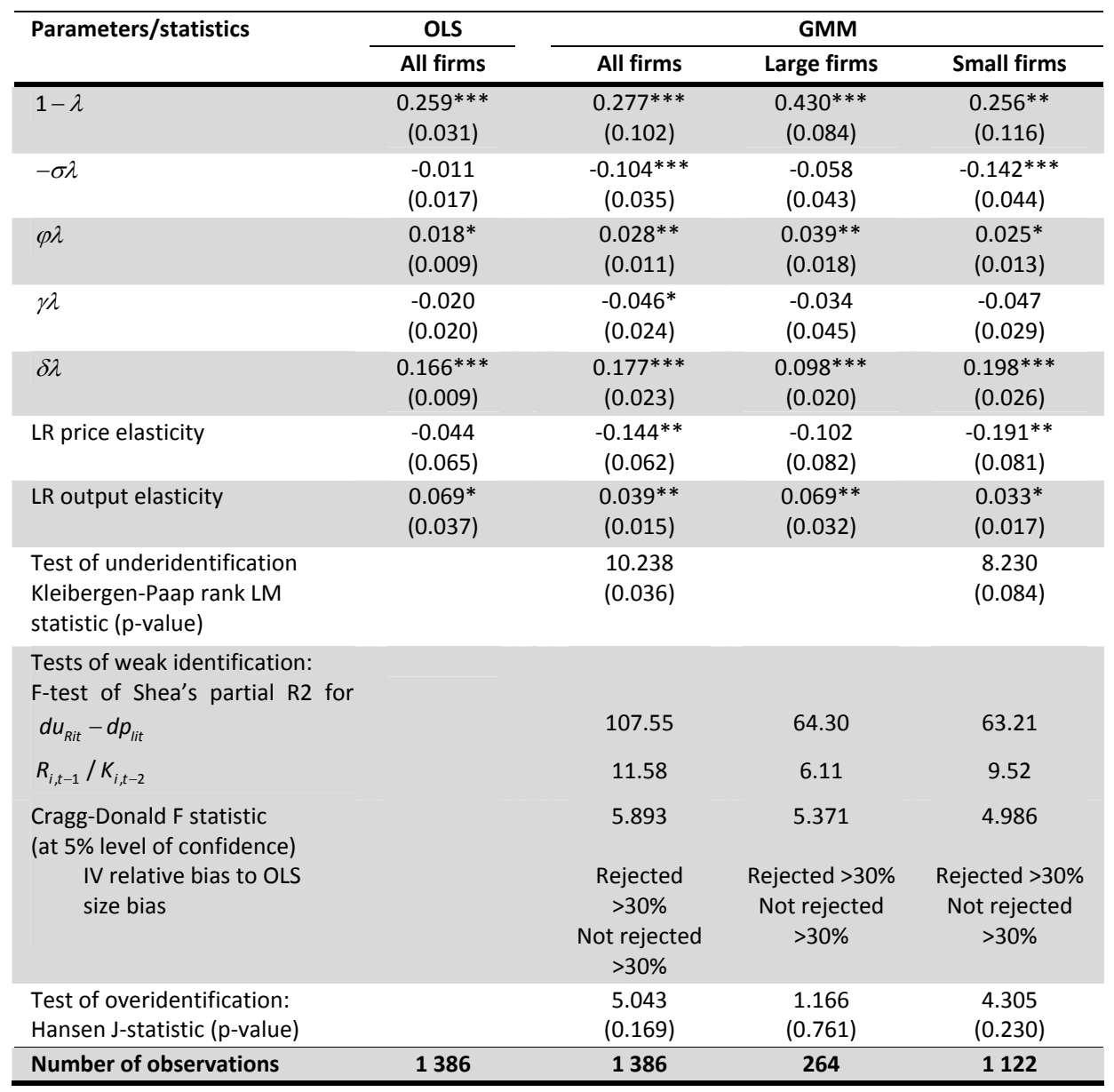

Notes: Estimation period is $1998-2003 . * * *$ indicates significance at $1 \%, * *$ at $5 \%, *$ at $10 \%$. Standard errors of the long-run elasticities are computed using the delta method. Heteroscedasticity consistent standard errors in parentheses unless otherwise indicated.

We have also experimented with an alternative modeling of the dynamics in the R\&D investment by specifying an error-correction model with an autoregressive distributed lag specification $\operatorname{ADL}(1,1)$ following Mairesse and Mulkay (2004). This specification does not assume a particular type of adjustement as we do in the above model. However, the alternative model did not yield a significant price elasticity of R\&D. Maybe a higher-order lagged specification would be required, but 
our short time interval did not allow us to do this. Finally, we could think of estimating a different sensitivity to R\&D tax credits between small and large firms by exploiting a regression discontinuity design, in the sense that the user cost of R\&D would be lower for small firms that benefit from more generous R\&D tax credits. The number of firms falling in a close neighbourhood of the discontinuity in the R\&D tax credit schedule, some falling just above and some just below \$2 ML of R\&D wages, was too small to find good matches to compare the treatment effect of both types of firm.

\subsection{Measuring Effectiveness of R\&D}

To evaluate the effectiveness of the whole R\&D tax incentive program we compare the present situation with a fictive scenario where the government changes part of the R\&D tax incentive scheme. In the absence of a proper cost-benefit calculation, including all indirect costs and benefits related to such a program (administration costs, opportunity costs and externalities), the usual way to assess the effectiveness of R\&D tax incentives consists in computing the so-called "bang for the buck" (BFTB). By that is meant how much private R\&D increases per dollar of $R \& D$ tax receipts foregone. If it is greater than $1, R \& D$ tax incentives are considered to be effective in stimulating additional R\&D; a value smaller than 1 means that part of the money received from tax incentives substitutes for private financing.

Table 5.5-1: $\quad$ Trajectory of R\&D flows and stocks under two scenarios

\begin{tabular}{lllll}
\hline & Old scenario & & $\begin{array}{l}\text { New scenario } \\
\text { (after a change in the R\&D tax credits) }\end{array}$ \\
\cline { 2 - 5 } periods & R\&D flow & $\begin{array}{l}\text { R\&D } \\
\text { stock }\end{array}$ & R\&D flow & R\&D stock \\
\hline 0 & $R_{0}$ & $K_{0}$ & $R_{0}$ & $K_{0}$ \\
\hline 1 & $R_{1}=\delta K_{0}+\left(K_{1}-K_{0}\right)$ & $K_{1}$ & $\tilde{R}_{1}=\delta K_{0}+\left(\tilde{K}_{1}-K_{0}\right)$ & $\tilde{K}_{1}=K_{1}+\left(\partial K_{1} / \partial u_{R}^{1}\right) \Delta u_{R}^{1}$ \\
\hline 3 & $R_{2}=\delta K_{1}+\left(K_{2}-K_{1}\right)$ & $K_{2}$ & $\tilde{R}_{2}=\delta \tilde{K}_{1}+\left(\tilde{K}_{2}-\tilde{K}_{1}\right)$ & $\tilde{K}_{2}=\tilde{K}_{1}+\left(K_{2}-K_{1}\right)+\left(\partial K_{2} / \partial u_{R}^{1}\right) \Delta u_{R}^{1}$ \\
\hline 5 & $R_{3}=\delta K_{2}+\left(K_{3}-K_{2}\right)$ & $K_{3}$ & $\tilde{R}_{3}=\delta \tilde{K}_{2}+\left(\tilde{K}_{3}-\tilde{K}_{2}\right)$ & $\tilde{K}_{3}=\tilde{K}_{2}+\left(K_{3}-K_{2}\right)+\left(\partial K_{3} / \partial u_{R}^{1}\right) \Delta u_{R}^{1}$ \\
\hline & $\cdot$ & $\cdot$ & $\cdot$ & $\cdot$ \\
\hline
\end{tabular}

Notes: The derivative $\partial K_{j} / \partial u_{R}$ represents the change in desired R\&D stock that occurs j-1 periods after the change in the user cost of R\&D caused by a change in the tax incentives in period 0 $\left(\Delta u_{R}^{0}\right)$. For simplicity, here the firm subscript $i$ has been omitted. 
To find out how much R\&D arises from an R\&D tax incentive scheme, we have to compute the differences in R\&D flows (or expenditures) from period 1 onwards till infinity between the two scenarios, where the flows of each additional year are discounted by $1 /(1+r)$ vis-à-vis the previous year. This in our particular model is equal to ${ }^{18}$

$$
\sum_{i} \sum_{t=1}^{\infty}\left(\tilde{R}_{i t}-R_{i t}\right) /(1+r)^{t-1}=\sum_{i} \sum_{t=1}^{\infty}\left[\delta\left(\frac{\partial K_{i 1}}{\partial u_{R}}+\ldots+\frac{\partial K_{i, t-1}}{\partial u_{R}}\right)+\frac{\partial K_{i t}}{\partial u_{R}}\right] \Delta u_{R i}^{0} /(1+r)^{t-1}
$$

where:

$$
\frac{\partial K_{i t}}{\partial u_{R i}}=-\sigma \lambda(1-\lambda)^{t-1} \frac{\tilde{K}_{i t}}{u_{R i, t}}
$$

where $-\sigma \lambda$ is the estimated short-run user cost elasticity of R\&D stock in the first period, $\lambda$ is the estimated partial adjustment coefficient, $\delta$ is the depreciation rate of the R\&D stock, taken to be $15 \%, r$ is the risk-free interest rate, taken to be on average $3 \%$, and where the user cost elasticity, common to all firms of a given size class and constant over time, is converted to a marginal effect for period $t$ using the optimal R\&D stock and the user cost of R\&D of period $t$.

For every dollar of private R\&D expenditure the government supports a fraction of it by giving tax incentives in proportion to the wage and salary costs for researchers, the fraction of $R \& D$ carried out by a public research centre, the fraction carried out in a research consortium, the fraction of pre-competitive research, or the increments of $R \& D$ with respect to a base year. Let us denote the fraction of the private $R \& D$ so supported by the tax incentive program for firm $i$ at year $t$ by $\gamma_{1} R_{i t}+\gamma_{2} \Delta R_{i t}$, where $\gamma_{1}$ is the R\&D tax credit rate in proportion of the level of R\&D and $\gamma_{2}$ the rate in proportion of the increase in R\&D. Table 5.A-1 lists for every year the average user cost of R\&D, the B-index and its components, and what the user cost of R\&D would be without the R\&D tax credit. For the various rates that apply in Québec, see Table 5.A-2.

The total cost to the government to support the R\&D tax credit program is given by

$$
\sum_{t=1}^{\infty}\left(\tilde{W}_{t}-W_{t}\right) /(1+r)^{t-1}=\sum_{i} \sum_{t}^{\infty}\left[\left(\gamma_{1} \tilde{R}_{i t}+\gamma_{2} \Delta \tilde{R}_{i t}\right)-\left(\gamma_{1} R_{i t}+\gamma_{2} \Delta R_{i t}\right)\right] /(1+r)^{t-1}
$$

The BFTB is given by the ratio of (5-10) and (5-12). In other words, we compute the ratio of the increases in $R \& D$ for all the firms along the entire

\footnotetext{
${ }^{18}$ See Lokshin and Mohnen (2007) for a detailed derivation of Equation ( 5-10).
} 
trajectories towards their new steady states to the saving in government costs to support R\&D along those trajectories, both appropriately discounted ${ }^{19}$.

In our computation of the BFTB we use size-class specific short and long-run estimated price elasticities reported in Section 5.4. We use two size classes: small firms with fewer than 250 employees, and large firms with 250 and more employees. We report each time the BFTB computed for all firms with the specificities in the tax scheme that pertain to them and with their own price elasticities. We also report the BFTB separately for small and large firms.

We run the following two experiments. In the first we increase the level-based provincial tax incentives $\left(\gamma_{1}\right)$ by $10 \%$ (all rates appearing in PL and PO are increased by $10 \%)$, in the other we increase the increment-based provincial R\&D tax credit $\left(\gamma_{2}\right)$ by $10 \%$. The result of the first experiment is plotted in Figure 5.5-1. For small firms the BFTB, i.e. the ratio of cumulative additional R\&D to cumulative government expenses to support R\&D, starts at a value much higher than one and slowly drops to a value that stays above 1 even after 20 years. Hence for small firms it is beneficial to support R\&D by tax incentives. More R\&D gets created than it costs the government to support it even after 20 years. For large firms, however, the BFTB starts at 3 but falls below 1. Already after seven years the cumulative additional $R \& D$ no longer covers the cumulative government costs to support this R\&D. If we take all the firms in our sample, the BFTB falls below the bar of one after 13 periods. Thus if we consider the whole adjustment towards a new steady state, the level-based R\&D tax incentives do not come out as very effective. The reason for this ineffectiveness is that level-based tax incentives contain a deadweight loss, namely the tax support on the R\&D that would be done in the absence of all support. The importance of the deadweight can be computed by rewriting (5-12) as:

$$
\sum_{i} \sum_{t}^{\infty}\left[\tilde{\gamma}_{1}\left(\tilde{R}_{i t}-R_{i t}\right)+\left(\tilde{\gamma}_{1}-\gamma_{1}\right) R_{i t}+\tilde{\gamma}_{2}\left(\Delta \tilde{R}_{i t}-\Delta R_{i t}\right)+\left(\tilde{\gamma}_{2}-\gamma_{2}\right) \Delta R_{i t}\right] /(1+r)^{t-1}
$$

The second term represents the deadweight loss connected to a change in the level-based R\&D tax credit. In percentage of the total, the deadweight loss converges to $68 \%$ for small firms and to $82 \%$ for large firms.

In the second experiment we increase only the incremental based R\&D tax credit by $10 \%$, i.e. the rate appearing in $\mathrm{PI}$. In this case, there can also be a deadweight loss, the fourth term in (5-13), to the extent that we do not start from an initial steady state but that some R\&D investment was already planned before

\footnotetext{
${ }^{19}$ Because of the unbalanced nature of our sample, t0 is different for every firm. We take it to be the last year a firm is observed in the sample. In the simulation for the removal of the incremental R\&D tax credit, we take as the reference year the last year this tax credit was in effect.
} 
the tax change. However, this deadweight loss is likely to be small. By simulating the effect of the tax change on R\&D and government support for R\&D for a period of 20 years, we find that the bang for the buck is about 2.98 for small firms and 2.79 for large firms. The incremental R\&D tax credit is much more effective than the level based tax credit, and its effectiveness does not vary very much by firm size.

Figure 5.5-1: Evolution of the bang for the buck when increasing by $10 \%$ the level-based provincial rate of R\&D tax credit

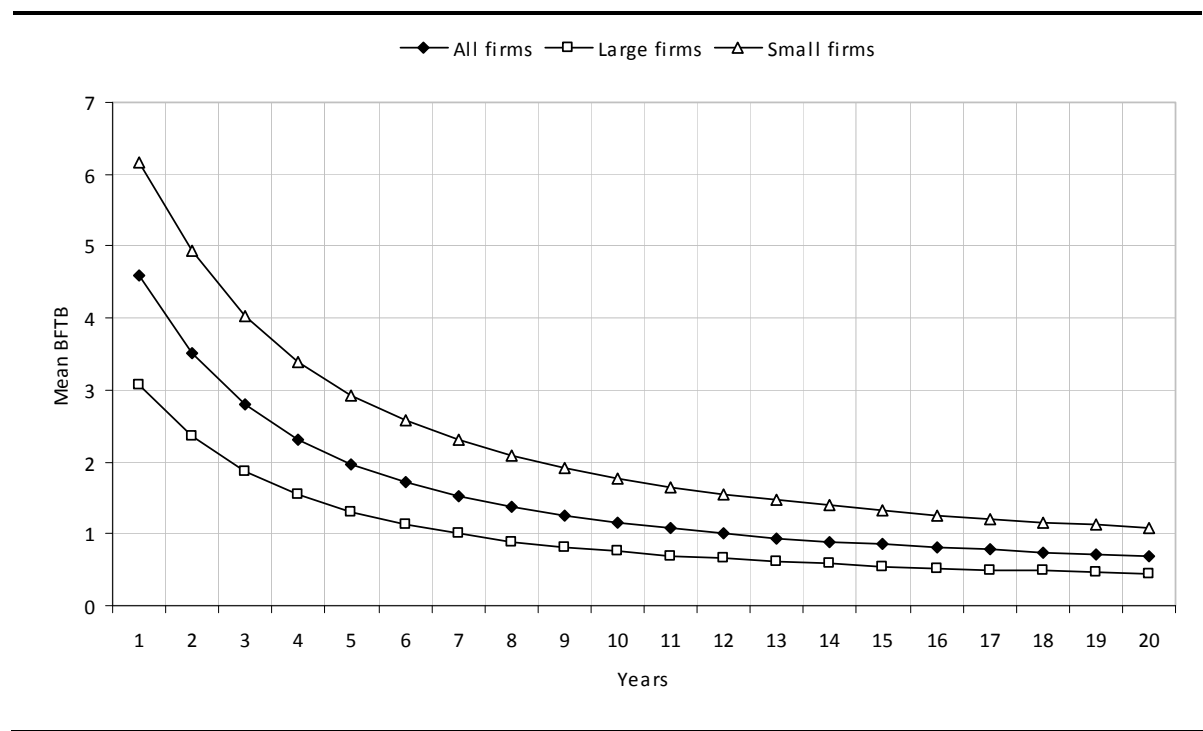

\subsection{Conclusion}

In this chapter we evaluate the effectiveness of R\&D tax incentives in Québec, using manufacturing firm data originating from R\&D surveys, annual surveys of manufactures and administrative data. The data cover the years 1997 to 2003 . We estimate the price responsiveness of $R \& D$ from a factor demand model for $R \& D$ with partial adjustment towards the steady state. From the estimated price elasticities we compute the bang for the buck for changes in the level-based and in the increment-based provincial R\&D tax credits. We are especially interested in comparing the effectiveness of R\&D tax incentives for small and large firms.

Small firms in Québec are slightly more responsive than large firms to price changes in the user cost of R\&D driven by various kinds of tax credits aimed at stimulating R\&D. Their estimated price elasticity of R\&D investment is 0.14 in the short run and 0.19 in the long run (in absolute value). The estimated short-run elasticities are in line with those estimated by Bernstein (1986), 0.13, and Dagenais et al. (2004), 0.07, for Canada. The long-run elasticities are larger in those studies 
(0.30 and 1.09 resp.) because of a faster estimated adjustment speed. Our estimated bang for the buck differs sizeably from those estimated by Hall (1993) and Mairesse and Mulkay (2004) on US and French data respectively. Apart from possible differences in modeling and in the definition of the bang for the buck, the main reason for their much higher estimated effectiveness ratios (above 2 ) is likely to be due to the exclusive use of incremental R\&D tax credits in France (until recently) and the United States. Our results on the differences between small and large firms regarding their sensitivities to $R \& D$ tax credits confirm the results on Dutch firms reported in Lokshin and Mohnen (2007).

We show that there is a deadweight loss associated with level-based R\&D tax incentives that is particularly acute for large firms. For small firms it is not sizeable enough to overcome the R\&D additionality, at least not during quite a number of years after the initial tax change. Incremental R\&D tax credits do not suffer from this deadweight loss and are from that perspective preferable to level-based tax incentives.

If it wants to run an efficient fiscal policy in support of R\&D, the province of Québec would be advised to continue using both the incremental R\&D tax credit and the level-based tax credits in favour of small firms. It is precisely small firms that have difficulty in getting outside financing for their R\&D efforts. Of course, effectiveness is not the only nor the ultimate goal in giving R\&D tax credits. An important consideration is the social rate of return on tax-supported R\&D. It would be interesting in future work to compare the social returns on government supported R\&D via tax credits and direct subsidies, on government supported and non-supported $R \& D$, and on $R \& D$ support for large and small firms. 


\section{Chapter Appendix}

\section{A. The User Cost of the R\&D in Québec}

The user cost of R\&D is given by $u_{R i}=p_{R}(r+\delta) B_{i t}$, where $p_{R}$ is the R\&D deflator, $r$ is the opportunity cost of funds, $\delta$ is the R\&D depreciation rate, and $B$ is the B-index given by:

$B_{i t}=\frac{1}{1-\tau}\left[1-P L_{i, t}-P O_{i, t}-P I_{i, t}-F C_{i, t}-P D_{i, t}-F D_{i, t}\right]$

where $P L_{i, t}, P O_{i, t}, P I_{i, t}, F C_{i, t}, P D_{i, t}, F D_{i, t}$ are defined as follows:

PL: Québec's SR\&ED tax credit and superdeductions for salaries and wages

For small firms (assets $<25000000)$

$P L_{i, t}=w_{i, t}^{t}\left\{\min \left(\frac{R_{p}}{W_{i, t}^{-} R_{i, t}}, 1\right)\left[\left(1-D_{1 j, t}\right) c_{p L, t}^{1}+D_{1, t} s_{L, t}^{1} \tau_{p, t}\right]+1_{R_{p} \triangleleft W_{i, t}^{t} R_{i, t}}\left(1-\frac{R_{p}}{w_{i, t}^{t} R_{i, t}}\right)\left[\left(1-D_{1 j, t}\right) c_{p L, t}^{2}+D_{1 j, t} s_{L, t}^{2} \tau_{p, t}\right]\right\}$

For medium sized firms (25 $000000<$ assets $<50000$ 000)

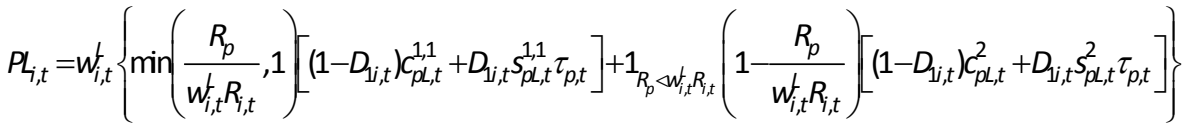

where $c_{p L, t}^{1,1}=c_{p L, t}^{1}-\left[\frac{\left(\text { Asset }_{i, t}-25000000\right)\left(c_{p L, t}^{1}-c_{p L, t}^{2}\right)}{25000000}\right]$

and $s_{p L, t}^{1,1}=s_{p L, t}^{1}-\left[\frac{\left(\text { Asset }_{i, t}-25000000\right)\left(s_{p L, t}^{1}-s_{p L, t}^{2}\right)}{25000000}\right]$

For large firms (assets $>50000000$ )

$P L_{i, t}=w_{i, t}^{L}\left[\left(1-D_{1 i, t}\right) c_{p L, t}^{2}+D_{1 i, t} s_{L, t}^{2} \tau_{p, t}\right]$

PO: Québec's tax credits and superdeductions for university research, precompetitive research and consortium research

$$
P O_{i, t}=\left(.8 w_{i, t}^{U n}+w_{i, t}^{P r}+w_{i, t}^{C o}-s_{i, t}^{E}\right)\left[\left(1-D_{2 i, t}\right) c_{p O, t}+D_{2 i, t} s_{p O, t} \tau_{p, t}\right]
$$


This holds for small as well as for large firms. In the case of tax credit and superdeductions for university research, eligible expenditures are fixed at $80 \%$ of the value of sub-contracted work.

$\mathrm{PI}$ : Québec's incremental tax credit

$$
P l_{i, t}=\left[\left(1-D_{3 i, t}\right) c_{p l, t}+D_{3 i} s_{p l, t} \tau_{p, t}\right]\left\{\left(1-w_{i, t}^{B}\right)-\left[\frac{1}{n} \sum_{1}^{n}\left(1-w_{i, t-n}^{B}\right) /(1+r)^{n}\right]\right\}
$$

FC: Federal SR\&ED tax credit

Small firms (assets $<25000000$ )

For federal SR\&ED tax credit, eligible expenditures are current R\&D expenditures and machinery and equipment expenditures.

Medium sized firms : $25000000<$ assets $<50000000$

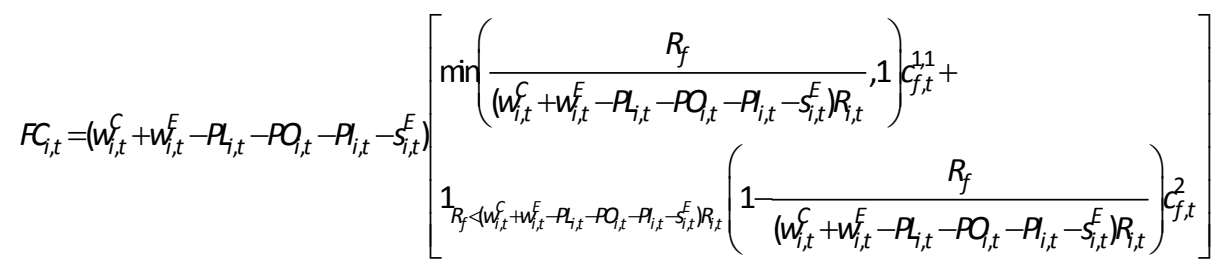

where $c_{f, t}^{1,1}=c_{f, t}^{1}-\left[\frac{\left(\text { Asset }_{i, t}-25000000\right)\left(c_{f, t}^{1}-c_{f, t}^{2}\right)}{25000000}\right]$;

Large firms ( assets $>50000000$ )

$$
F C_{i, t}=\left(w_{i, t}^{C}+w_{i, t}^{E}-P L_{i, t}-P O_{i, t}-P I_{i, t}-s_{i, t}^{E}\right) c_{f, t}^{2} ;
$$

PD : Québec's R\&D expensing

$$
P D_{i, t}=\left(w_{i, t}^{C}+w_{i, t}^{E} \theta z^{E}+w_{i, t}^{B} z^{B}-F C_{i, t}-s_{i, t}^{E}\right) \tau_{p, t} ;
$$

FD : Federal R\&D expensing

$$
F D_{i, t}=\left(w_{i, t}^{C}+w_{i, t}^{E} z^{E}+w_{i, t}^{B} z^{B}-F C_{i, t}-P L_{i, t}-P O_{i, t}-P I_{i, t}-s_{i, t}^{E}\right) \tau_{f, t}
$$

We assume that all firms are profitable, i.e. that they are able to take full advantage of all R\&D tax credits and deductions. $P L_{i, t}, P O_{i, t}$ and $P I_{i, t}$ are obtained not by the above formulas, but by dividing the observed tax credit payments 
corresponding to these items by the corresponding R\&D. $F C_{i, t}$ is computed using the statutory tax credit rates as in the above formula.

Table 5.A-1 decomposes the user cost of R\&D into its various components. All symbols and some key parameter values are given in Table 5.A-2.

Table 5.A-1: $\quad$ Estimated annual average user ost of R\&D, all firms, 1997-2003

\begin{tabular}{|c|c|c|c|c|c|c|c|c|c|c|}
\hline Year & Firms & $\boldsymbol{P}_{R t}(r+\delta)$ & $\mathrm{PL}_{i t}$ & $\mathrm{PO}_{i t}$ & $\mathrm{PI}_{i t}$ & $\mathrm{PC}_{i t}$ & $\mathrm{FC}_{i t}$ & $\mathrm{~B}_{i t}$ & $u_{r, i t}$ & $\begin{array}{r}u_{r, i t} \text { w/o } \\
\text { tax } \\
\text { credits }\end{array}$ \\
\hline & (1) & (2) & (3) & (4) & (5) & (6) & (7) & (8) & (9) & (10) \\
\hline 1997 & 1168 & 0.172 & 0.249 & 0.002 & 0.000 & 0.251 & 0.209 & 0.516 & 0.089 & 0.168 \\
\hline 1998 & 1265 & 0.183 & 0.243 & 0.003 & 0.000 & 0.246 & 0.208 & 0.523 & 0.096 & 0.179 \\
\hline 1999 & 1304 & 0.187 & 0.267 & 0.003 & 0.000 & 0.270 & 0.217 & 0.485 & 0.091 & 0.182 \\
\hline 2000 & 1909 & 0.197 & 0.271 & 0.005 & 0.050 & 0.326 & 0.197 & 0.462 & 0.091 & 0.194 \\
\hline 2001 & 2092 & 0.166 & 0.288 & 0.002 & 0.058 & 0.349 & 0.207 & 0.405 & 0.067 & 0.159 \\
\hline 2002 & 2400 & 0.176 & 0.299 & 0.002 & 0.057 & 0.357 & 0.208 & 0.393 & 0.069 & 0.169 \\
\hline 2003 & 2657 & 0.179 & 0.269 & 0.001 & 0.055 & 0.325 & 0.220 & 0.417 & 0.075 & 0.172 \\
\hline
\end{tabular}

Note: $\quad u_{r, i t}$, the user cost of R\&D in column (9) is the product of column (2) and $B_{i t}$, the B- index in column (8). $\mathrm{PL}_{i t}, \mathrm{PO}_{i t}, \mathrm{Pl}_{i t}, \mathrm{FC}_{i t}$ and the $\mathrm{B}_{i t}$ are described above. $\mathrm{PC}_{i t}$ in column (6) is the sum of columns (3) to (5), i.e. all forms of R\&D tax credits in Québec. Column (10) is the user cost of $R \& D$ without provincial and federal R\&D tax credits. 
Table 5.A-2: $\quad$ Chapter symbols and parameter values

\begin{tabular}{|c|c|c|}
\hline Variable & Value $^{a}$ & Description \\
\hline Asset $_{i, t}$ & & Asset of firm $i$ in year $t$ \\
\hline cf1 & $35 \%$ & First bracket rate for federal SR\&ED tax credit \\
\hline $\mathrm{cf} 2$ & $20 \%$ & Second bracket rate for federal SR\&ED tax credit \\
\hline $\mathrm{cpl}$ & $15 \%$ & Québec' s incremental R\&D tax credit rate \\
\hline cpL1 & $\begin{array}{l}\text { 1997-2002: } 40 \% \\
\text { 2003: } 35 \%\end{array}$ & $\begin{array}{l}\text { First bracket rate for Québec's salaries of researchers tax } \\
\text { credit }\end{array}$ \\
\hline $\mathrm{cpL} 1,1$ & $\begin{array}{l}1997-2002: 40 \% \text { to } 20 \% \\
2003: 35 \% \text { to } 17.5 \%\end{array}$ & $\begin{array}{l}\text { First bracket variable rate for Québec's salaries of researchers } \\
\text { tax credit applying to firms with assets between } \$ 25000000 \\
\text { and } \$ 50000000\end{array}$ \\
\hline $\mathrm{cpL} 2$ & $\begin{array}{l}\text { 1997-2002: } 20 \% \\
\text { 2003: } 17.5 \%\end{array}$ & $\begin{array}{l}\text { Second bracket rate for Québec's salaries of researchers tax } \\
\text { credit }\end{array}$ \\
\hline $\mathrm{cpO}$ & $\begin{array}{l}\text { 1997-2002: } 40 \% \\
\text { 2003: } 35 \%\end{array}$ & $\begin{array}{l}\text { Québec's tax credit rate for university research, } \\
\text { precompetitive research and dues paid to consortium }\end{array}$ \\
\hline $\mathrm{D} 1_{i, t}$ & $\begin{array}{l}-\mathrm{D} 1 \mathrm{i}, \mathrm{t}=1 \\
-\mathrm{D} 1 \mathrm{i}, \mathrm{t}=0\end{array}$ & $\begin{array}{l}\text {-R\&D performer claimed superdeduction for salaries } \\
\text {-R\&D performer claimed tax credit for salaries }\end{array}$ \\
\hline $\mathrm{D} 2_{i, t}$ & $\begin{array}{l}-D 2 i, t=1 \\
-D 2 i, t=0\end{array}$ & $\begin{array}{l}\text {-R\&D performer claimed superdeduction for university, } \\
\text { precompetitive or consortium research } \\
\text {-R\&D performer claimed tax credit for university, } \\
\text { precompetitive or consortium research }\end{array}$ \\
\hline $\mathrm{D} 3_{i, t}$ & - D3i,t =1 & $\begin{array}{l}\text {-R\&D performer claimed superdeduction based on the } \\
\text { increase in } R \& D \text { expenditures } \\
\text {-R\&D performer claimed tax credit based on the increase in } \\
R \& D \text { expenditures }\end{array}$ \\
\hline $\mathrm{Rf}$ & $\$ 2000000$ & First bracket ceiling for federal SR\&ED tax credit \\
\hline $\begin{array}{l}\mathrm{R}_{i, t} \\
\mathrm{Rp}\end{array}$ & $\$ 2000000$ & $\begin{array}{l}\text { Total R\&D spending of firm } i \text { in year } t \\
\text { First bracket ceiling for Québec's salaries of researchers 'tax } \\
\text { credit }\end{array}$ \\
\hline si,t E & & $\begin{array}{l}\text { Proportion of Québec's subsidies for equipment in total R\&D } \\
\text { of firm } i \text { in year } t\end{array}$ \\
\hline $\mathrm{spl}, \mathrm{t}$ & $190 \%$ & Québec's superdeduction incremental rate \\
\hline $\mathrm{spL},, \mathrm{t} 1$ & $460 \%$ & $\begin{array}{l}\text { First bracket rate for Québec's superdeduction for salaries } \\
\text { and wages }\end{array}$ \\
\hline $\mathrm{spL}, \mathrm{t}$ 1,1 & between $460 \%$ and $230 \%$ & $\begin{array}{l}\text { First bracket variable rate for Québec' s superdeduction for } \\
\text { salaries and wages applying to firms with assets between CAN } \\
\$ 25 \mathrm{ML} \text { and } 50 \mathrm{ML}\end{array}$ \\
\hline $\mathrm{spL},, \mathrm{t} 2$ & $230 \%$ & $\begin{array}{l}\text { Second bracket rate for Québec's superdeduction for salaries } \\
\text { and wages }\end{array}$ \\
\hline $\mathrm{spO}, \mathrm{t}$ & $460 \%$ & $\begin{array}{l}\text { Québec' s superdeduction for university, pre-competitive and } \\
\text { consortium research }\end{array}$ \\
\hline$\theta$ & Applied in 1999: $125 \%$ & $\begin{array}{l}\text { Depreciation deduction rate of the acquisition costs of } \\
\text { machinery and equipment }\end{array}$ \\
\hline$\tau f$ & $\begin{array}{l}\text { SMEs: } 22,12 \%^{b} \\
\text { Large firms: } 22,12 \%\end{array}$ & Federal corporate income tax rate \\
\hline$\tau p$ & $\begin{array}{l}\text { SME's: } 8,90 \%{ }^{\mathrm{c}} \\
\text { Large firms: } 8,90 \%\end{array}$ & Québec's corporate income tax rate \\
\hline$\tau$ & $\tau f+\tau p$ & $\begin{array}{l}\text { Combined federal and province of Québec corporate income } \\
\text { tax rate }\end{array}$ \\
\hline Wi,tB & & Proportion of land and building expenses in total R\&D \\
\hline Wi,tC & & Proportion of current $R \& D$ expenditure in total $R \& D$ \\
\hline Wi,tCo & & Proportion of dues paid to a research consortium \\
\hline
\end{tabular}




\begin{tabular}{ll}
\hline Variable $\quad$ Value $^{\mathrm{a}}$ & Description \\
\hline $\mathrm{Wi}, \mathrm{tE}$ & Proportion of machinery and equipment expenses in total \\
$\mathrm{Wi}, \mathrm{tL}$ & R\&D \\
$\mathrm{Wi}, \mathrm{tPr}$ & Proportion of labor costs in total R\&D \\
$\mathrm{Wi}, \mathrm{tUn}$ & Proportion of precompetitive research project expenses \\
$\mathrm{zB}$ & Proportion of university research contract expenses \\
$\mathrm{zE}$ & Present value of depreciation of R\&D building \\
\hline
\end{tabular}

\section{Notes:}

a The values of the variables given in the table are limited to the study period i.e., for the years 1997 to 2003. The subscripts $i$ and $t$ represent the firm and time, respectively.

${ }^{b}$ For the years 1997 to 2002 , a federal corporate income tax rate of $13.12 \%$ applied to the first $\$ 200000$ of the taxable income of SME's. In 2003, the ceiling of \$200 000 was raised to $\$ 225000$.

'For the years 1997 and 1998, a Québec's corporate income tax rate of $5.75 \%$ applied to the first $\$ 400000$ of the taxable income of SME's. 



\title{
CHAPTER 6
}

\author{
Public R\&D Subsidies and \\ Productivity: Evidence from Firm- \\ Level Data in Québec
}

Submitted for publication 


\subsection{Introduction}

Most countries now recognize the need for supporting and strengthening innovation activities in order to ensure productivity growth and hence long-term prosperity and competitiveness in the global economy. Neo-classical theory explains the need for government involvement in technological advance and innovation activities by the Arrow-Nelson rationale, which is the key argument of the neoclassical approach of market failure (Nelson (1959), Arrow (1962)). Essentially, the market failure approach point to the underinvestment from private firms in R\&D leading to a supply offer of knowledge in the economy that is less than what would be socially desirable. In an effort to fill this gap, several governments implemented various incentive programs targeted to private firms such as fiscal-based frameworks or more direct public schemes such as grants, loans, procurements, venture capital investments or other instruments.

In Canada, as in most OECD countries, the orientation taken in the last decades by governments shows a clear tendency towards fiscal incentives such as tax credits. This choice has been motivated mainly by the distinctive feature of tax credits of being neutral with respect to the choice of the industry or of projects. However, this neutrality also has the unattractive feature that companies tend to choose systematically the projects with the highest rates of return leaving so aside the least profitable projects. For this reason, it is not surprising that many governments spend every year, in addition to their fiscal incentive programs, several millions of dollars in specific direct subsidy programs to firms in order to increase private R\&D spending in targeted industries or in projects that would not be carried out without some type of subsidy. Some authors recommend rather the use of both policy instruments in a more integrated approach since there is evidence that tax incentives stimulate mainly $R \& D$ projects that involve more applied or short-term research while direct subsidies affect projects which research is more basic or long-run (Guellec and Van Pottelsberghe (2000)). In any case, the question of the goal attainment of the objectives at which direct subsidies in a context where fiscal incentives are already present may be asked. In particular, this refers mainly to the two following questions: First, do public R\&D grants result in increased R\&D spending (input additionality) from firms that already receive tax credits and, second, what is the impact of these publicly funded R\&D on productivity growth? This chapter proposes to add to the evidence on this literature by exploring empirically these questions using longitudinal data from manufacturing firms in Québec in the range 1997-2004.

To date, these research questions have not been previously investigated with data from Québec and, at the Canadian level, there are only a few studies that 
address the effectiveness of public schemes to R\&D and, not surprisingly, they all focus on R\&D tax credits given the emphasis of the Canadian government support programs on the latter ${ }^{20}$. The only Canadian studies that address the effectiveness of subsidies are the studies by Pagé (1995) and Bérubé and Mohnen (2009), but in these cases too, the focus is not on the input additionality of public R\&D grants nor in their impact in firms' productivity. These studies focus rather on innovation output. To the benefit of this chapter, the methodological approach used by Bérubé and Mohnen (2009) has however a realistic feature in that, by evaluating the effectiveness of public R\&D grants, it takes into account the effects of the other public incentives such as tax credits. The present chapter draws on this methodological approach for assessing the input additionality of public R\&D grants. There exist a considerable body of research on the two main questions addressed in this chapter using data from other countries, but they traditionally have been treated separately, i.e. there are, on the one hand, studies that address the question of the additionality of public subsidies and in most cases they did not take into account the effects of tax credits in country with both type of government incentives. The evidence from these studies is mixed and this casts doubt on the question whether public subsidies increase firms' private spending in R\&D or to the contrary, crowds out this spending. Klette, Moen and Griliches (2000) or David, Hall and Toole (2000) provide good reviews of these studies. On the other hand, there are studies that analyze the impact of firms' total R\&D spending (privately and publicly funded) on productivity growth. Most of them estimate an elasticity of the output with respect to the R\&D effort or a rate of return to firms' R\&D expenditures. In the latter case, on which this chapter focuses, empirical estimates of the rate of return vary between $20 \%$ and $50 \%$. Examples include Griliches and Mairesse (1991), Wakelin (2001), Smith et al. (2004) and Maté-Garcia and Rodriguez-Fernandez (2008).

The novel aspect of this empirical study is that it combines the two research questions into one analysis by taking advantage of a set of rich microdata from manufacturing firms in Québec. More specifically, to answer the first question we use a semiparametric matching estimator, more specifically the conditional semiparametric difference-in-differences estimator and, following Bérubé and Mohnen (2009), we focus on the comparison between the intensity of R\&D of firms that receive $R \& D$ grants in addition to $R \& D$ tax credits and the intensity of $R \& D$ of firms that receive only $R \& D$ tax credits. Hence, the estimated differences in the intensity of R\&D of both groups permit to assess the input additionality of R\&D grants in the presence of tax credits. For the second question, we subsequently use these estimated differences in a production function controlling also for other

\footnotetext{
${ }^{20}$ See McFetridge and Warda (1983), Mansfield and Switzer (1985b), Mansfield and Switzer (1985a), Bernstein (1986), Lebeau (1996), Dagenais et al. (2004), Dahlby (2005), Czarnitzki et al. (2005), Baghana and Mohnen (2009) and Baghana (2008).
} 
variables in order to evaluate the impact of R\&D grants to firm's growth productivity.

The paper is organized as follows: Section 6.2 provides an overview of the R\&D tax incentives in Québec. Section 6.3 sketches the methodological approach which is undertaken in two steps: The first step regarding the matching estimator framework from which we shall assess the input additionality of R\&D grants and the second step regarding the production function framework from which we shall assess the impact of privately and publicly funded R\&D on productivity growth. In Section 6.4, we first describe briefly the three data sources used and the merging process of these data before explaining variables construction. Section 6.5 presents the empirical analysis and the results. Section 6.6 concludes after a discussion.

\subsection{Public Direct Subsidies toward R\&D in Québec}

Over the last thirty years, most of the governmental financial assistance to R\&D activities in Québec from both the Government of Québec and the Canadian government, has been granted to firms in the form of fiscal incentives through Québec's and federal scientific research and experimental development programs and this has resulted in a significant decrease in the amounts of grants awarded annually to firms. However, both governments maintained through the years a few direct funding programs to R\&D in addition to their SR\&ED programs. At the provincial level there is no agency dedicated to the management of public funding programs. Financial assistance is attributed to firms by the ministries and some government agencies. In most cases, the assistance from these institutions is granted for R\&D expenditures that do not entitle firms to claim tax credits from SR\&ED. The Ministère du Développement économique, de I'Innovation et de I'Exportation is the main provincial institution for public direct funding to firms ${ }^{21}$. In addition to the grants from the Government of Québec, firms can also apply for federal grants through the Industrial Research Assistance Program (NRC-IRAP) which is the most important federal program administered by National Research Council of Canada. Firms may also benefit from grants or contracts programs from other federal agencies acting in specific areas such as energy or military (e.g. Mines and Resources Grants, Canadian Space Agency Grants, Defence Industry Productivity Program). More details on the description of direct incentives in Québec can be found in Chapter 3.

\footnotetext{
${ }^{21}$ This role was previously played by the ministère de l'Industrie et du Commerce (MIC) and the ministère de la Recherche, de la Science et de la Technologie (MRST) which were merged in 2003 to form the ministère du Développement économique et régional et de la Recherche. The latter was replaced in turn by the MDEIE from 2006.
} 
Table 6.2-1 shows the evolution in the period 1997-2004 of R\&D grants and contracts from both the Canadian federal government and the Government of Québec to manufacturing firms performing R\&D activities. The overall value of grants and contracts dropped from $\$ 100$ million to only $\$ 7$ million in 2004 and this fall originates essentially in a reduction of grants and contracts from the Canadian government between 1997 and 2001. Such a fall points out to policies adopted by the federal and provincial governments in Canada in the last years and reflects the preference over time by both levels of government for fiscal incentives policy over $R \& D$ grants incentives. This is illustrated clearly by observing the evolution of the ratio of the combined federal-provincial R\&D grants and contracts to the combined federal-provincial tax credits received by firms in this period as indicated in the last line of Table 6.2-1. From more than $46 \%$ in 1997, this ratio was no longer more than $2.2 \%$ in 2004.

Table 6.2-1: Comparative evolution of public direct incentives and tax credits aimed at R\&D in the manufacturing sector in Québec, 1997-2004

\begin{tabular}{lrrrrrrrr}
\hline & \multicolumn{7}{c}{ (\$ millions) } \\
\cline { 2 - 8 } & 1997 & 1998 & 1999 & 2000 & 2001 & 2002 & 2003 & 2004 \\
\hline $\begin{array}{l}\text { Provincial and federal } \\
\text { R\&D grants }\end{array}$ & & & & & & & & \\
$\quad$ Provincial & 3 & 4 & 2 & 3 & 5 & 11 & 4 & 4 \\
$\quad$ Federal & 97 & 36 & 30 & 20 & 5 & 5 & 3 & 3 \\
\hline $\begin{array}{l}\text { Total R\&D grants } \\
\text { R\&D grants / Tax } \\
\text { credits }\end{array}$ & $\mathbf{1 0 0}$ & $\mathbf{4 0}$ & $\mathbf{3 2}$ & $\mathbf{2 3}$ & $\mathbf{1 0}$ & $\mathbf{1 6}$ & $\mathbf{7}$ & $\mathbf{7}$ \\
\hline
\end{tabular}

Sources: Ministère du Revenu du Québec and Statistics Canada, calculations by the authors.

Note: Calculations in this table have been done from the data of the Survey of Research and Development in Canadian Industry linked to the administrative data from the Ministère du Revenu du Québec. Firms that could not be matched in both samples were discarded. These firms represent about $9.5 \%$ of the total number of firms.

Table 6.2-2 and Table 6.2-3 summarize the use of the public grants by size class and by technology level. Table 6.2-2 reveals an asymmetric distribution of R\&D grants between large and small firms. Indeed, more than $87 \%$ of R\&D recipients are small firms (less than 250 employees) with only $12.9 \%$ of the total value of attributed R\&D grants. However, $82.8 \%$ of the total amount of attributed R\&D grants goes to the largest firms (more than 500 employees) present in the sample in the proportion of only $8.6 \%$. The pattern of this asymmetric distribution is also comparable to that of the use of tax credits by firms in the manufacturing sector as stated by Baghana and Mohnen (2009) and refers to questions concerning firms accessibility to public funding. When questioned about this issue, most companies' 
administrators of the small enterprises invoke reasons such as the complexity of the application procedure of the government programs, the lack of time and human resources necessary to follow up technological activities, and the high consultancy that exceed in some cases the benefits of the program, etc.

Table 6.2-2: $\quad$ Use of public grants by size class in the manufacturing sector, 1997-2004

\begin{tabular}{|c|c|c|c|c|c|}
\hline & \multirow{2}{*}{$\begin{array}{r}\% \text { Grants } \\
n\end{array}$} & \multicolumn{3}{|c|}{ Value of grants } & \multirow{2}{*}{$\begin{array}{c}\text { Ratio grants /tax } \\
\%\end{array}$} \\
\hline & & $\%$ & $\%$ federal & Total & \\
\hline$\geq 500$ employees & 8.6 & 43.2 & 89.9 & 82.8 & 17.2 \\
\hline 250 to 499 employees & 4.2 & 11.5 & 3.0 & 4.3 & 4.9 \\
\hline 50 to 249 employees & 28.5 & 29.6 & 3.8 & 7.7 & 3.2 \\
\hline$<50$ employees & 58.7 & 15.7 & 3.3 & 5.2 & 2.3 \\
\hline TOTAL & 100.0 & 100.0 & 100.0 & 100.0 & 9.6 \\
\hline
\end{tabular}

Sources: Ministère du Revenu du Québec and Statistics Canada, compiled by the Institut de la Statistique du Québec and the authors.

Turning now to the distribution of R\&D grants by technology group in the manufacturing sector, it can be stated in Table 6.2-3 that, more than $74.4 \%$ of the firms in the sample are in groups other than the high technology group making up only $15.1 \%$ of the total value of attributed R\&D grants. However, the high technology group which represents $25.6 \%$ of the total number of R\&D grant recipients gets almost $85.0 \%$ of the total amount of attributed R\&D grants. Clearly, these differences suggest that most federal and provincial direct support programs are used by the high technology group which is R\&D-intensive.

Table 6.2-3: $\quad$ Use of public grants by technology level ${ }^{22}, 1997-2004$

\begin{tabular}{|c|c|c|c|c|c|}
\hline & \multirow{2}{*}{$\frac{\text { \% Grants }}{n}$} & \multicolumn{3}{|c|}{ Value of grants } & \multirow{2}{*}{$\frac{\text { Ratio grants /tax }}{\%}$} \\
\hline & & $\%$ & $\%$ federal & Total & \\
\hline High-technology & 25.6 & 41.6 & 92.8 & 84.9 & 16.5 \\
\hline Medium-high-technology & 30.7 & 21.0 & 4.9 & 7.4 & 4.6 \\
\hline Medium-low-technology & 34.5 & 35.3 & 1.9 & 7.0 & 2.3 \\
\hline Low-technology & 9.2 & 2.1 & 0.4 & 0.7 & 1.2 \\
\hline TOTAL & 100.0 & 100.0 & 100.0 & 100.0 & 9.6 \\
\hline
\end{tabular}

Sources: Ministère du Revenu du Québec and Statistics Canada, compiled by the Institut de la Statistique du Québec and the authors.

\footnotetext{
22 Based on OECD classification of manufacturing industries using International Standard Industrial Classification (ISIC) rev.3 activity breakdown (see classification in Table 6.D-4).
} 


\subsection{Methodological Approach}

The methodology comprises two steps, each investigating one of the two main research questions of this chapter, i.e. whether public $R \& D$ grants result in increased R\&D spending from firms (input additionality), and what is the impact of privately and publicly funded R\&D on productivity growth. In the first step, input additionality is evaluated by computing an overall average effect on knowledge capital using the individual effects for each recipient firm estimated by the semiparametric matching estimator. The estimated individual effects are then subtracted from the firm's knowledge capital in order to isolate, on the one hand, the knowledge capital induced by $R \& D$ expenditures funded out of firms' own pockets and by R\&D tax credits and, on the other hand, the knowledge capital induced by R\&D expenditures funded by public grants. In the second step, a productivity growth function is estimated and the two knowledge capital components from the first step are included along with other variables as regressors in the estimation. This two-step approach has been previously used by Czarnitzki and Licht (2006). However, our study is departing from theirs by the matching scheme used. Furthermore, their study is based on a different specification for the production function as it focuses on innovation output. Indeed, they introduce the two knowledge capital components resulting from the matching process as innovation input in a Griliches invention production function linking innovation output to innovation input. The focus of this chapter is rather on the growth productivity of firms.

Before describing in detail the matching estimator and the production function frameworks used respectively in the first and second step of the methodological approach, let's first present formally this two-step approach. As a starting point, consider a prototypical model of economic choice. Let $Y_{i}^{0}$ and $Y_{i}^{1}$ denote two potential outcomes of the firm $i$ respectively with treatment and without treatment, that is, in our case, the outcome of firm $i$ if it claims only tax credits and its outcome when it receives both tax credits and $R \& D$ grants. $T_{g i}=\{0,1\}$, is the indicator of exposure to treatment that takes the value 1 if the firm is treated and 0 otherwise. We can write the following:

$$
\begin{array}{ll}
Y_{i}^{0}=h^{0}\left(X_{i}\right)+\varepsilon_{i}, \quad \text { if } T_{g i}=0 & (i=1, \ldots, n) \\
Y_{i}^{1}=h^{1}\left(X_{i}\right)+\tau_{i}+\varepsilon_{i}, & \text { if } T_{g i}=1
\end{array}
$$

where $\tau_{i}$ is the impact or the effect of the treatment to be estimated. The aggregation of these individual effects yields the widely known ATT (average treatment effect on the treated). For the estimation of this effect, we adopt the 
approach of the matching estimator. The advantage of using the matching estimator is that it allows $h(X)$ having an unknown functional form rather than being, for instance, a linear combination of $X$ such as in the case of parametric sample selection models.

For a firm $i$, the gain from treatment is given by:

$Y_{i}^{1}-Y_{i}^{0}=\tau_{i}$

In the second step, a productivity function $Q$ is estimated using the following regressors: $Y_{i}^{0}=Y_{i}^{1}-\tau_{i}$, i.e. the knowledge capital, taken as R\&D intensity, induced by private $R \& D$ expenditures and by $R \& D$ tax credits, $\tau_{i}$, the knowledge capital induced by $R \& D$ expenditures funded by public grants and other variables $Z_{i}$ affecting productivity. This relationship is given by:

$Q_{i}=f\left(Y_{i}^{0}, \tau_{i}, Z_{i}\right)$

\subsubsection{Step 1: The Matching Estimator Framework}

\subsubsection{The Fundamental Evaluation Problem and the Selection Bias Problem}

The estimation of Equation (6-2) is not straightforward because only one of the two possible outcomes is observed at the same time i.e. $Y_{i}^{0}$ or $Y_{i}^{1}$. In other words, $Y=T_{g i} Y_{i}^{1}+\left(1-T_{g i}\right) Y_{i}^{0}$. This is known in the literature as the fundamental evaluation problem.

In order to solve this issue, treatment impacts measures are approximated by means impacts. Hence, Equation (6-2) is rewritten as the average treatment effect on the treated firms (ATT) and is given by:

$$
A T T=E\left(Y^{1} \mid T_{g}=1, X\right)-E\left(Y^{0} \mid T_{g}=1, X\right)
$$

where $X$ is a set of comparable characteristics. If the mean $E\left(Y^{1} \mid T_{g}=1, X\right)$ is known, it is not the case for $E\left(Y^{0} \mid T_{g}=1, X\right)$ the no-treatment outcome of program participants. This unknown outcome may be approximated by the outcome of the nonparticipants $E\left(Y^{0} \mid T_{g}=0, X\right)$ if such information is available. However, another important issue arises when using this approximation. Indeed, unless the case of randomly selected firms, the outcome of treated firms in case of non-treatment is expected to differ from the outcome of nonparticipants i.e. 
$E\left(Y^{0} \mid T_{g}=1, X\right) \neq E\left(Y^{0} \mid T_{g}=0, X\right)$. As a consequence, Equation (6-4) using such an approximation can possibly be biased. This problem is known as the selection bias. Several models in the statistics and econometrics literature propose identifying assumptions to estimate $E\left(Y^{0} \mid T_{g}=1, X\right)$ i.e. what program participants would experience had they not participated.

\subsubsection{The Matching Methods}

To address the fundamental evaluation problem and the selection bias problem, different models referred to as models of economic choice have been proposed, among them are the matching estimators. Matching is based, as stated by Heckman, Ichimura and Todd (1997), on the intuitively attractive idea of contrasting the outcomes of program participants with the outcomes of "comparable" nonparticipants. There are two approaches to matching, first, the parametric matching approach and, second, the non parametric approach to matching. While in the former approach one needs to estimate consistently the two counterfactuals' situations by assuming specific functional forms, the latter approach, which is used in this chapter, has the advantage of not requiring doing so.

From the introduction of the hypothesis of conditional independence assumption (CIA) by Rubin (1977), non parametric matching methods have been improved and became one of the most important models used in the evaluation literature especially in the labor market area. The CIA states that for individuals with the same set of exogenous characteristics $\mathrm{V}$, the participation and the potential outcome are independent. When this condition is satisfied, the selection bias is eliminated and the outcome of treated firms in case of non-treatment equals the outcome of nonparticipants that is, $E\left(Y^{0} \mid T_{g}=1, X\right)=E\left(Y^{0} \mid T_{g}=0, X\right)$. The CIA is expressed as follows:

$\left(Y^{1}, Y^{0}\right) \perp T_{g} V$

where $\mathrm{V}$ is the multidimensional vector of pre-treatment characteristics. This condition states the independence of outcomes $\left(Y^{1}, Y^{0}\right)$ from the treatment variables given a set of conditioning variables $V$. We recall that the vector $V$ is formed of observable variables $X$ that affect the outcomes of other observable variables $Z$ determining choices. However, the non-parametric matching methods do not make any distinction between the $X$ and $Z$ variables.

In order to control for the multidimensional problem, Rosenbaum and Rubin (1983) introduced in their seminal paper the method of propensity score matching. Propensity score is defined as the probability that a treatment was assigned to a 
unit given information in the control variables. In other words, it is the probability that an individual takes treatment. Matching on the propensity is a useful alternative because it reduces the multidimensional problem to one dimension. Formally, the propensity score is given by:

$$
p(V)=\operatorname{Pr}\left\{T_{g}=1 \mid V\right\}=E\left\{T_{g} \mid V\right\}
$$

To derive this equation, Rosenbaum and Rubin (1983) assume the following:

Lemma 1: Balancing of pre-treatment variables given the propensity score. If $p(V)$ is the propensity score, then

$$
\left(T_{g} \perp V \mid p(V)\right.
$$

Lemma 2: Unconfoundedness given the propensity score. Suppose that assignment to treatment is unconfounded, i.e.

$$
\left(Y^{1}, Y^{0}\right) \perp T_{g} V
$$

Then under (5-1) and (6-8) it can be shown that assignment to treatment is unconfounded given the propensity score, i.e.

$$
\left(Y^{1}, Y^{0}\right) \perp T_{g} \mid p(V)
$$

The propensity score $p(V)$ can be estimated using any standard probability model. Many matching estimators based on propensity score have been developed for the estimation of the average effect of treatment (ATT), which is the most used evaluation parameter. A generalized form for the ATT may be written as follows:

$$
\tau_{A T T}=\frac{1}{N_{1}} \sum_{i \in(T g=1)}\left[Y_{i}^{1}-\sum_{j \in(T g=0)} w_{i j} Y_{j}^{0}\right]
$$

where $w_{i j}$ is a weight that is function of the propensity score $p(V)$. This weight function gives higher weights to nonparticipants $j$ with propensity score closer to that of the participant $i$ and a lower weight to nonparticipants $j$ with distant propensity score. Hence, for all $i, \sum_{j \in(T g=0)} w_{i j=1}=1$. In this setting, the treated are matched with a weighted average of all controls with weights that are inversely proportional to the distance between the propensity scores of treated and controls. 
The most known matching estimators are Nearest neighbor matching, Radius matching estimator (Cochran and Rubin (1973)), Kernel matching and Local linear regression matching estimator. These estimators differ from each other by the weights they attach to individuals of the comparison group. A good review of matching estimators can be found in Smith and Todd (2003).

These traditional cross-section matching estimators however have been criticized since they do not allow controlling for the potential bias that may arise from unobservable time trends which are common across treatment and comparison groups. Indeed, if the outcome variable change over time due to reasons unrelated to the participation decision, these estimators will be biased. Various alternate estimators have been proposed to eliminate this potential bias and one of the most compelling estimators is the difference-in-differences estimator (DiD) (see for example Heckman and Robb (1985)). The DiD is based in the simple idea of subtracting the before-after change in nonparticipant outcomes from the before-after change in the participant outcome. Hence, relying on the assumption of time-invariant linear selection, this double-difference permits to eliminate the bias from common time trends. Reformulating Equation (6-10) accordingly, we get the DiD estimator :

$$
\tau_{\text {DiD }}=\frac{1}{N} \sum_{i \in\left(T_{g}=1\right)}\left[Y_{i, t 1}^{1}-Y_{i, t 0}^{1}-\sum_{j \in\left(T_{g}=0\right)} w_{i j}\left(Y_{j, t 1}^{0}-Y_{j, t 0}^{0}\right)\right]
$$

The application of the simplest DiD estimator is based on the simple comparison of the outcomes of the treatment group and of the control group between pre-treatment period to and post-treatment period t1. Heckman, Ichimura, Smith and Todd (1998a) introduced an estimator that combines this standard DiD estimator with any of the matching estimators based on propensity score mentioned above, namely the conditional semiparametric difference-indifferences estimator (CDiD). More specifically, the CDiD estimator is implemented using Equation (6-11), but in this case the weights $w_{i j}$ are based on the propensity score $p(V)$. This is the key difference and the advantage of the CDiD over the standard DID since, by using $\mathrm{p}(\mathrm{V})$ in Equation (6-11), the CDiD allows not only selection on both observables and on unobservables, but it also resolves the multidimensional problem mentioned above. Another advantage of the CDiD matching estimator is that, unlike traditional cross-section matching estimators, it combines in one step the matching process with the estimation of the effect of the treatment. Smith and Todd (2005) show that the CDiD estimator is more robust than standards DiD estimators. The CDiD may be based on any of the various propensity matching estimators previously cited. 
Following Heckman et al. (1998a), we apply in this chapter the CDiD with weights based on the local linear regression matching estimator (LLR henceforth). These weights are defined by Heckman et al. (1998a) as:

$$
w_{i j}=\frac{G_{i j} \sum_{k \in(T g=0)} G_{i k}\left(p_{k}-p_{i}\right)^{2}-\left[G_{i j}\left(p_{j}-p_{i}\right)\right]\left[\sum_{k \in(T g=0)} G_{i k}\left(p_{k}-p_{i}\right)\right]}{\sum_{j \in(T g=0)} G_{i j} \sum_{k \in(T g=0)} G_{i j}\left(p_{k}-p_{i}\right)^{2}-\left(\sum_{k \in(T g=0)} G_{i k}\left(p_{k}-p_{i}\right)\right)^{2}}
$$

where $\mathrm{p}$ is the propensity score, $G_{i j}=G\left(\left(p_{i}-p_{j}\right) / a_{N}\right)$ is a kernel function and $a_{N}$ is a bandwidth parameter ${ }^{23}$. Intuitively, $w_{i j}$ uses all the observations in the control group reservoir and places higher weight on units close in terms of propensity score $p$ and lower weights on more distant observations. Local linear weights are superiors to any conventional kernel weights since local linear estimators converge at a faster rate at boundary points and adapt better to different data densities (Heckman et al. (1997), Fan (1992)) ${ }^{24}$.

To summarize, for the application of the CDiD estimator, we apply Equation (6-11) using the weights defined by Equation (6-12). For each treated firm we obtain at each period an estimate of the effect of the treatment $\tau_{i, t}$, now defined by $\tau_{D i D, i, t}$ and given by:

$$
\tau_{i, t}=\tau_{D i D, i, t}=f\left(Y_{i, t}^{1}, Y_{i, t-1}^{1}, Y_{j, t}^{0}, Y_{j, t-1}^{0}, w_{i j}(p)\right)
$$

\subsubsection{Step2: The Production Function Framework}

To assess the effectiveness of the public direct support in the growth productivity of firms, we use a production function linking output to physical capital, to labour and to knowledge capital. The latter, which is our variable of interest, enters the production function in two separate components, that is on the one hand, the share of knowledge capital induced by $R \& D$ expenditures funded privately and by tax credits, and on the other hand, the share of knowledge capital induced by R\&D expenditures funded by public grants. These two components are taken from the estimation of the average effect of treatment described in the previous section.

\footnotetext{
${ }^{23}$ The bandwidth parameter $a_{N}$ is assumed to converge toward zero as $n$ and $n a_{N}$ converge toward infinity.

${ }^{24}$ It is worth noting that LLR matching estimator is a generalized version of the kernel matching estimator and both are special cases of local polynomial estimators (Cleveland (1979, Stone (1977)).
} 
For the production function, let's consider a typical a Cobb-Douglas production function given by:

$$
Q_{i t}=A e^{\lambda_{t}} K_{i t}^{\alpha} L_{i t}^{\beta} S_{i t}^{\prime \gamma^{\prime}} S^{\prime \prime}{ }_{i t}^{\prime \prime} e^{\varepsilon_{i t}}
$$

where i denotes firms and t years, $Q$ is the output, $K$ is physical capital, $L$ is labour, $S^{\prime}$ is the composite share of knowledge capital induced by R\&D expenditures privately funded and funded by tax credits, $S$ "is the share of knowledge capital induced by R\&D expenditures funded by public grants, $A$ is a constant, $\lambda$ is a scale factor measuring the rate of disembodied technical change, $\alpha$ is the elasticity of output with respect to physical capital, $\beta$ the elasticity of output with respect to labour, $\gamma^{\prime}$ the elasticity of output with respect to knowledge capital induced by private R\&D and by tax credits, $\gamma^{\prime \prime}$ the elasticity of output with respect to knowledge capital induced by public grants, and finally $\varepsilon$ is the error term that takes account of all the other factors such as measurement errors, firms technological heterogeneity and all other factors not accounted for explicitly in the inputs.

For the estimation of Equation (6-14), since in the first step of the chapter we used the R\&D intensity as a proxy for the knowledge capital, Equation (6-14) must be transformed in such a way that it includes R\&D intensity in its functional form instead of knowledge capital. This is fulfilled by adapting to our needs the derivation proposed by Griliches (1973) into a labour productivity equation as follows ${ }^{25}$ :

$$
\Delta(q-I)_{i t}=\lambda+\alpha \Delta(k-I)_{i t}+\theta \Delta I_{i t}+\rho^{\prime}\left(\frac{R^{\prime}}{Q}\right)_{i t}+\rho^{\prime \prime}\left(\frac{R^{\prime \prime}}{Q}\right)_{i t}+v_{i t}
$$

where lower case letters denote the logarithms of variables. $\rho^{\prime}$ is the rate of return on R\&D induced by private R\&D and tax credits, and $\rho^{\prime \prime}$ is the rate of return induced by public grants. In the same manner, $\left(\frac{R^{\prime}}{Q}\right)$ and $\left(\frac{R^{\prime \prime}}{Q}\right)$ are respectively the $R \& D$ intensity induced by private $R \& D$ and tax credits, and by public grants. $\theta=\alpha+\beta-1$ is the measure of returns to scale and, if we assume constant returns to scale, this measure should equal zero. However, if this measure is less than or greater than zero, then decreasing, respectively increasing, returns to scale prevail.

\footnotetext{
${ }^{25}$ The labour productivity equation is an extended version of the Cobb-Douglas production function based on the proposition of Griliches (1973) See Appendix 6.E for the full derivation of this equation.
} 


\subsection{Data and Variables}

\subsubsection{Data}

The data consist of a longitudinal sample of 3821 manufacturing firms over the period 1997-2004 (11 884 observations) obtained by linking three micro data files. Such linking was necessary since only a part of the required information is available in each file. The first file is sourced from the Statistics Canada annual Survey of Research and Development in Canadian Industry $(\mathrm{RDCl})^{26}$ which is a census with cross sectional design that collects data on all Canadian firms known or believed to perform or fund R\&D. In order to reduce the reporting burden on firms, only firms performing or funding more than $\$ 1$ million in R\&D are surveyed. For all the other firms, the data are extracted from administrative data from Canada Revenue Agency. For the purpose of this analysis, the main information collected from this file is the information on public subsidies. Indeed, the RDCl survey is the only source of information that collects information on the various types of government direct incentives (grants, loans, procurements, venture capital investments or other instruments) from both provincial governments and the federal government including its agencies. The second file comes from the ASM Survey. Data collected directly from firms' respondents by questionnaires and from administrative files from CRA permit to compile financial information such as employment, wages, total cost of materials, total sales of manufactured products, inventories, value added by manufacturing and capital expenditures. The third linked file comes from administrative data from Revenu Québec that collects fiscal data from R\&D performers claiming tax credits located in Québec or have R\&D conducted on their behalf in Québec. Hence, the addition of this file to our dataset allowed us to gather records of the amounts of SR\&ED or other fiscal incentives to R\&D from the Government of Québec effectively received by firms ${ }^{27}$.

For both the RDCl data survey and the Revenu Québec's administrative data, the reporting unit is the company or enterprise. However, the reporting unit in the case of the ASM survey is the establishment. We grouped the establishments in the ASM data file by enterprise in the cases of multi-establishments prior to performing the linking of the three data file. After matching the three files, discarding cases of firms' merger and acquisition and cleaning outliers in the data, we obtained a sample of 3815 firms with 11842 observations of which, 485 cases received both

\footnotetext{
${ }^{26}$ Detailed description of the RDCl survey can be found in the Industrial Research and Development (2002 Intentions).

${ }^{27}$ Only the information related to SR\&ED and other fiscal incentives to R\&D from the Government of Québec was available to us. However, by using official formulas and both the RDCl data and the Revenu Québec's administrative data, we estimated the amounts of fiscal incentives to R\&D received by firms from the federal government. This procedure is explained in more details in Baghana and Mohnen (2009).
} 
tax credits and R\&D grants and, the remaining 11357 cases received tax credits only. However, it should be noted that because of the methodological approach adopted, the matching sample used corresponds to the years 1998 to 2004 with 3749 firms (11 012 observations, of which 410 are treated and 10602 are nontreated). Detailed description of this sample is presented in Section 6.5.1.

\subsubsection{Variables for the Estimation of ATT}

\subsubsection{Treatment Variable}

The treatment variable, denoted $T_{g}$, is a dummy variable that takes the value of 1 if a firm received both $R \& D$ tax credits and $R \& D$ grants from either the Canadian government or from the Government of Québec. In the case where the firm received only $R \& D$ tax credits from either the Canadian government or from the Government of Québec, the treatment variable takes the value of 0 . Thus, $T_{g}$ is a binomial variable that captures the program participation status of a firm and is, for that, the dependant variable in the regression for the estimation of the propensity score.

\subsubsection{Selection Variables}

We take the variables that influence both the probability, for a firm that receives tax credits, of getting an R\&D grant (or R\&D contract) and the investment in private $R \& D$. These selection variables are taken at the beginning of the period prior to the decision to participate (or not), that is in our case, the first lag of these variables. As our dataset is rich enough, we included most variables often used to estimate this probability ${ }^{28}$ : Firm size is widely considered as one of the variables that affect positively this probability and the latter is likely higher for larger firms. Indeed, larger firms are less constrained by factors such as fixed cost barrier to R\&D, the lack of time and human resources necessary to follow up technological activities. We use the lagged log of the number of employees $\left((I)_{t-1}\right)$ as proxy for the size of the firm. The intensity of capital $\left((k / I)_{t-1}\right)$, constructed as the lag of the logarithm of firm's real assets divided by the number of employees, is added to capture the influence of the capital structure in the willingness of firms to engage in R\&D. The share of technical personnel working in R\&D activities (engineers, scientists) is a good indicator of firms' involvement in innovation activities. We therefore added a

\footnotetext{
${ }^{28}$ We present only variables retained in the final estimation. Other variables that were not statistically significant were discarded in the final estimations. Among these variables are variables often used in the literature: age of firm, control variables for market competition such as the concentration ratio or the import ratio that captures the competitive pressure of foreign firms on the market (see for example Almus and Czarnitzki (2003)).
} 
variable constructed as the lagged value of the ratio of firm's R\&D personnel to number of employees $\left(\left(L_{r} / L\right)_{t-1}\right)$. Almus and Czarnitzki (2003) point out the importance of also including variables that control for market competition. For that, we use respectively, the market share $\left(\left(q / q_{l}\right)_{t-1}\right)$, constructed as the lagged value of the ratio of firm's real sales to industry's real sales (at 3-digit NAICS classification) in logarithm and the export share $\left(\left(e / q_{1}\right)_{t-1}\right)$ constructed as the lagged value of the ratio of firm's real exportations to industry's real sales in logarithm. We include in the regression a country of control dummy (OWN) that equals 1 if the country of control of the firm is not Canada and 0 otherwise. It is often advanced in the literature that a firm with a foreign owned parent company will usually not apply for grants when its demand in R\&D is adequately supplied by the latter. If this is true for most of the firms owned by a foreign company, we should expect a negative sign in the coefficient of the dummy OWN. We control for technological differences by including twenty dummies (indus1-indus20) to take account of the firm specific effects in the twenty one sectors of the NAICS at the 3 digit code level (see NAICS classification in Table 6.D-5).

\subsubsection{Outcome Variable}

Sometimes referred to as response variables, outcome variables are the outputs of models of economic choice such as the model described in Section 6.3.1. The response variable of interest in this chapter is the intensity of $R \& D$ constructed as the ratio of firm's real R\&D expenditures $R$ to firm's output $Q$. In what follows, we denote it $R / Q$.

\subsubsection{Variables Entering the Production Function}

The main variables entering the production function are those appearing explicitly in Equation (6-15) described in Section 6.3.2, i.e. the labour productivity growth rate $\left(\Delta(q / I)_{t}\right)$, the capital-labour growth rate $\left(\Delta(k / I)_{t}\right)$, the R\&D intensity induced by private $R \& D$ and tax credits $\left(R^{\prime} / Q\right)_{t}$, the $R \& D$ intensity induced by public grants $\left(R^{\prime \prime} / Q\right)_{t}$ and the employment growth rate $\left(\Delta I_{t}\right)$.

In addition to these variables, we add to the regression other variables in order to control the effects of factors not explicitly accounted for. Six indicator variables denoted $t 1$ to $t 6$ are added to take account of time effects in the study period (1998-2004) and 20 group effect dummies as described in the previous section. 


\subsection{Empirical Analysis and Results}

\subsubsection{Assessing the Input Additionality of R\&D Grants}

To assess the input additionality of R\&D subsidies in Québec, we investigated whether public subsidies induce firms in Québec to spend more on R\&D than they would without the grants. To do so, we estimated the treatment effect of public grants on firms R\&D level using the CDiD matching estimator described in Section 6.3.1. Since the choice is given to firms by the provincial and federal governments to apply each year for grants in addition to the tax credits they are entitled to claim, the treatment effect of public grants should also be evaluated each year. Hence, the CDiD matching estimator is applied at each period of the matching sample (i.e. from $t=1998$ to $t=2004$ ) and, for the group of treated firms, a treatment effect $\tau_{(D i D, t)}$ is obtained for each treated period. The treatment effect is not evaluated for the first year of the original sample, i.e. 1997, due to the presence of lagged variables in the estimation of the propensity score and also because, for each evaluation point, the difference-in-differences estimator requires data for at least two periods.

As another goal of this chapter is to see how firms of different technological level respond to public grants, we also performed matching analysis using two subsamples of technology level breakdown (Low-medium technology and High technology). Table 6.5-1 shows the description of the samples used in the matching for the full sample and for the two technologies sub-samples.

To obtain the propensity score, for each of the seven periods, we estimated and compared several specifications based on probit and on logit models with different set of conditioning variables. We ended-up choosing a specification based on a probit model with the following conditioning variables: the lagged log number of employees, the lagged log capital intensity, the lagged share of R\&D personnel, the lagged log market share, the lagged log export share and the degree of foreign ownership. This specification was clearly superior to the logit model with the same set of covariates since it showed better statistical goodness of fit and lower value for the well-known Akaike's Information Criterion for selecting among nested econometric models.

Table 6.5-2 shows the results of probit models for all firms estimated for each year from 1998 to 2004 with the corresponding sub-sample. The results for the lowmedium technology firms and for the high technology firms are in Table 6.5-3 and Table 6.5-4 respectively. A look at the three tables reveals that all the probit regression models are statistically significant (All firms: LR Chi2 $(1998-2004)=[21.35$ 38.81], $P=[0.000$ 0.002]; Low-Medium technology firms: LR Chi2 (1998-2004) = [13.86 30.69], $P=$ [0.000 0.031]; High technology firms: LR Chi2 (1998-2004) = [13.95 26.74], $P=[0.0000 .030])$. On the other hand, all the predictor variables in 
the models in the three samples are statistically significant at the $10 \%$ level and, across the three samples; they have the same sign at each point of time. More specifically, this indicates that the probability to get a subsidy increases with the number of employees, the capital intensity and the share of R\&D personnel and decreases with the market share, the export share and the degree of foreign ownership.

Table 6.5-1: Description of the samples used in the matching

\begin{tabular}{|c|c|c|c|c|c|c|}
\hline \multirow[t]{2}{*}{ Samples } & \multicolumn{2}{|c|}{ All firms } & \multicolumn{2}{|c|}{$\begin{array}{l}\text { Low and medium- } \\
\text { technology }\end{array}$} & \multicolumn{2}{|c|}{ High technology } \\
\hline & $\mathrm{N}_{1}$ & $\mathrm{~N}_{0}$ & $\mathrm{~N}_{1}$ & $\mathrm{~N}_{0}$ & $\mathrm{~N}_{1}$ & $\mathrm{~N}_{0}$ \\
\hline 1998 & 58 & 819 & 38 & 726 & 20 & 93 \\
\hline 1999 & 56 & 886 & 41 & 770 & 15 & 116 \\
\hline 2000 & 76 & 1565 & 60 & 1385 & 16 & 180 \\
\hline 2001 & 56 & 1542 & 41 & 1352 & 15 & 190 \\
\hline 2002 & 58 & 1925 & 48 & 1728 & 10 & 197 \\
\hline 2003 & 54 & 2032 & 40 & 1825 & 14 & 207 \\
\hline 2004 & 52 & 1833 & 39 & 1654 & 13 & 179 \\
\hline
\end{tabular}

Note: $\quad \mathrm{N}_{1}=$ Number of treated firms (firms with tax credits + R\&D grants), $\mathrm{N}_{0}=$ Number of non-treated firms (firms with tax credits only). 
Table 6.5-2: Probit estimates for the propensity score estimation, all firms, 1998-2004

\begin{tabular}{|c|c|c|c|c|c|c|c|}
\hline \multirow{2}{*}{$\begin{array}{l}\text { Parameters / } \\
\text { Statistics }\end{array}$} & \multicolumn{7}{|c|}{ Probit } \\
\hline & 1998 & 1999 & 2000 & 2001 & 2002 & 2003 & 2004 \\
\hline \multirow{2}{*}{$(I)_{t-1}$} & $0.351 * * *$ & $0.196 * * *$ & $0.289 * * *$ & $0.376 * * *$ & $0.346 * * *$ & $0.430 * * *$ & $0.322 * * *$ \\
\hline & $(0.115)$ & $(0.063)$ & $(0.086)$ & $(0.090)$ & $(0.093)$ & $(0.088)$ & $(0.097)$ \\
\hline \multirow{2}{*}{$(k / I)_{t-1}$} & $0.167 * *$ & $0.0805^{* *}$ & $0.0910 * *$ & $0.145^{*}$ & $0.252^{* *}$ & $0.154 *$ & $0.187^{*}$ \\
\hline & $(0.071)$ & $(0.040)$ & $(0.046)$ & $(0.077)$ & $(0.121)$ & $(0.092)$ & $(0.107)$ \\
\hline \multirow{2}{*}{$\left(L_{r} / L\right)_{t-1}$} & $1.221 * * *$ & $1.406^{* * *}$ & $1.655^{* * *}$ & $0.386^{* * *}$ & $0.266^{*}$ & $1.028^{* * *}$ & $0.951 * * *$ \\
\hline & $(0.470)$ & $(0.459)$ & $(0.533)$ & $(0.143)$ & (0.144) & $(0.325)$ & $(0.348)$ \\
\hline \multirow{2}{*}{$\left(q / q_{l}\right)_{t-1}$} & $-0.243^{* *}$ & $-0.110^{*}$ & $-0.252^{* * *}$ & $-0.195^{* * *}$ & $-0.165^{* *}$ & $-0.244^{* * *}$ & $-0.175^{* *}$ \\
\hline & $(0.098)$ & $(0.057)$ & $(0.068)$ & $(0.064)$ & $(0.074)$ & $(0.077)$ & $(0.084)$ \\
\hline \multirow[b]{2}{*}{$\left(e / q_{1}\right)_{t-1}$} & $-0.0464 * *$ & $-0.173^{* *}$ & $-0.0571^{*}$ & $-0.178 * *$ & $-0.0824^{*}$ & $-0.0336^{*}$ & $-0.240 *$ \\
\hline & $(0.023)$ & $(0.085)$ & (0.033) & $(0.074)$ & $(0.045)$ & $(0.018)$ & $(0.132)$ \\
\hline \multirow[t]{2}{*}{ OWN } & $-0.619 * *$ & $-0.632^{* *}$ & $-0.789 * * *$ & $-0.340 * *$ & $-0.313^{*}$ & $-0.474 * * *$ & $-0.263^{*}$ \\
\hline & $(0.286)$ & $(0.276)$ & $(0.284)$ & $(0.150)$ & $(0.165)$ & & \\
\hline $\begin{array}{l}\text { Industry } \\
\text { dummies } \\
\text { indus1- } \\
\text { indus20 }\end{array}$ & included & included & included & included & included & included & included \\
\hline Intercept & $\begin{array}{c}-5.400 * * * \\
(1.522)\end{array}$ & $\begin{array}{c}-6.047 * * * \\
(1.248)\end{array}$ & $\begin{array}{c}-6.223 * * * \\
(1.357)\end{array}$ & $\begin{array}{c}-5.977 * * * \\
(1.527)\end{array}$ & $\begin{array}{c}-6.953 * * * \\
(2.003)\end{array}$ & $\begin{array}{c}-5.668 * * * \\
(1.851)\end{array}$ & $\begin{array}{c}-6.406 * * * \\
(1.936)\end{array}$ \\
\hline Pseudo $\mathrm{R}^{2}$ & 0.092 & 0.080 & 0.081 & 0.083 & 0.073 & 0.090 & 0.074 \\
\hline Log likelihood & -141.21 & -133.89 & -153.67 & -185.34 & -162.88 & -176.74 & -172.71 \\
\hline $\begin{array}{l}\text { Number of } \\
\text { observations }\end{array}$ & 647 & 662 & 849 & 1140 & 1315 & 1473 & 1447 \\
\hline
\end{tabular}

Notes: $\quad * * *$ indicates significance at $1 \%, * *$ at $5 \%, *$ at $10 \%$. Standard errors in parentheses unless otherwise indicated. Lower case symbol denote the logarithm of the corresponding variable. $I$ :log of number of employees, $k / I$ : capital intensity, $L_{r} / L$ : share of R\&D personnel, $q / q_{l}$ : market share, $e / q_{1}$ : export share, OWN: origin country dummy (Canada=1, Foreign=0), indus1-indus20: industry dummies. 
Table 6.5-3: Probit estimates for the propensity score estimation, Low-medium technology firms, 1998-2004

\begin{tabular}{|c|c|c|c|c|c|c|c|}
\hline \multirow{2}{*}{$\begin{array}{l}\text { Parameters / } \\
\text { statistics }\end{array}$} & \multicolumn{7}{|c|}{ Probit } \\
\hline & 1998 & 1999 & 2000 & 2001 & 2002 & 2003 & 2004 \\
\hline$(/)_{t-1}$ & $\begin{array}{l}0.307^{* *} \\
(0.138)\end{array}$ & $\begin{array}{c}0.164 * * \\
(0.070)\end{array}$ & $\begin{array}{l}0.203^{* *} \\
(0.084)\end{array}$ & $\begin{array}{c}0.304^{* * *} \\
(0.108)\end{array}$ & $\begin{array}{c}0.283^{* * *} \\
(0.093)\end{array}$ & $\begin{array}{c}0.388^{* * *} \\
(0.104)\end{array}$ & $\begin{array}{l}0.177^{* *} \\
(0.089)\end{array}$ \\
\hline$(k / I)_{t-1}$ & $\begin{array}{l}0.183^{*} \\
(0.097)\end{array}$ & $\begin{array}{l}0.107 * * \\
(0.051)\end{array}$ & $\begin{array}{c}0.0969 * \\
(0.055)\end{array}$ & $\begin{array}{l}0.220^{*} \\
(0.126)\end{array}$ & $\begin{array}{l}0.143^{*} \\
(0.077)\end{array}$ & $\begin{array}{l}0.184^{*} \\
(0.107)\end{array}$ & $\begin{array}{l}0.195^{*} \\
(0.100)\end{array}$ \\
\hline$\left(L_{r} / L\right)_{t-1}$ & $\begin{array}{l}2.193^{*} \\
(1.202)\end{array}$ & $\begin{array}{l}1.263^{* *} \\
(0.591)\end{array}$ & $\begin{array}{l}1.042^{*} \\
(0.596)\end{array}$ & $\begin{array}{l}0.346^{*} \\
(0.206)\end{array}$ & $\begin{array}{l}0.238^{*} \\
(0.143)\end{array}$ & $\begin{array}{l}0.783 * * \\
(0.377)\end{array}$ & $\begin{array}{c}0.999 * * * \\
(0.379)\end{array}$ \\
\hline$\left(q / q_{l}\right)_{t-1}$ & $\begin{array}{c}-0.274 * * \\
(0.112)\end{array}$ & $\begin{array}{c}-0.132^{* *} \\
(0.066)\end{array}$ & $\begin{array}{c}-0.255^{* * *} \\
(0.067)\end{array}$ & $\begin{array}{c}-0.169 * * * \\
(0.065)\end{array}$ & $\begin{array}{c}-0.136 * * \\
(0.066)\end{array}$ & $\begin{array}{c}-0.262^{* * *} \\
(0.091)\end{array}$ & $\begin{array}{l}-0.155^{* *} \\
(0.075)\end{array}$ \\
\hline$\left(e / q_{1}\right)_{t-1}$ & $\begin{array}{l}-0.131^{*} \\
(0.079)\end{array}$ & $\begin{array}{c}-0.0745^{*} \\
(0.044)\end{array}$ & $\begin{array}{c}-0.0623^{*} \\
(0.036)\end{array}$ & $\begin{array}{l}-0.148 * \\
(0.080)\end{array}$ & $\begin{array}{c}-0.120 * * * \\
(0.038)\end{array}$ & $\begin{array}{c}-0.0373 * * \\
(0.018)\end{array}$ & $\begin{array}{c}-0.389 * * \\
(0.152)\end{array}$ \\
\hline OWN & $\begin{array}{l}-0.625^{*} \\
(0.372)\end{array}$ & $\begin{array}{l}-0.402^{*} \\
(0.242)\end{array}$ & $\begin{array}{l}-0.638^{*} \\
(0.360)\end{array}$ & $\begin{array}{c}-0.345^{* *} \\
(0.176)\end{array}$ & $\begin{array}{l}-0.325^{*} \\
(0.191)\end{array}$ & $\begin{array}{c}-0.337^{*} \\
(0.187)\end{array}$ & $\begin{array}{l}-0.432 * \\
(0.242)\end{array}$ \\
\hline $\begin{array}{l}\text { Industry dummies } \\
\text { indus1-indus } 20\end{array}$ & included & included & included & included & included & included & included \\
\hline Intercept & $\begin{array}{c}-6.260 * * * \\
(2.046)\end{array}$ & $\begin{array}{c}-5.907^{* * *} \\
(1.129)\end{array}$ & $\begin{array}{c}-5.691^{* * *} \\
(1.485)\end{array}$ & $\begin{array}{c}-6.211 * * * \\
(1.691)\end{array}$ & $\begin{array}{c}-2.629 * * \\
(1.300)\end{array}$ & $\begin{array}{c}-5.949 * * * \\
(2.144)\end{array}$ & $\begin{array}{c}-6.017^{* * *} \\
(1.370)\end{array}$ \\
\hline Pseudo $\mathrm{R}^{2}$ & 0.063 & 0.067 & 0.073 & 0.065 & 0.065 & 0.078 & 0.089 \\
\hline Log likelihood & -92.31 & -98.31 & -121.94 & -144.35 & -140.26 & -138.69 & -129.76 \\
\hline $\begin{array}{l}\text { Number of } \\
\text { observations }\end{array}$ & 548 & 564 & 734 & 981 & 1159 & 1308 & 1285 \\
\hline
\end{tabular}

Notes: $\quad * * *$ indicates significance at $1 \%, * *$ at $5 \%, *$ at $10 \%$. Standard errors in parentheses unless otherwise indicated. Lower case symbol denote the logarithm of the corresponding variable. $I$ :log of number of employees, $k / I$ : capital intensity, $L_{r} / L$ : share of R\&D personnel, $q / q_{l}$ : market share, $e / q_{l}$ : export share, OWN: origin country dummy ( Canada=1, Foreign=0), indus1-indus20: industry dummies. 
Table 6.5-4: Probit estimates for the propensity score estimation, High technology firms, 1998-2004

\begin{tabular}{|c|c|c|c|c|c|c|c|}
\hline \multirow{2}{*}{$\begin{array}{l}\text { Parameters / } \\
\text { statistics }\end{array}$} & \multicolumn{7}{|c|}{ Probit } \\
\hline & 1998 & 1999 & 2000 & 2001 & 2002 & 2003 & 2004 \\
\hline$(I)_{t-1}$ & $\begin{array}{c}0.430 * * * \\
(0.133)\end{array}$ & $\begin{array}{c}0.308^{* * *} \\
(0.116)\end{array}$ & $\begin{array}{l}1.231^{* *} \\
(0.500)\end{array}$ & $\begin{array}{c}0.486^{* *} \\
(0.210)\end{array}$ & $\begin{array}{c}0.720 * * * \\
(0.271)\end{array}$ & $\begin{array}{c}0.801^{* * *} \\
(0.311)\end{array}$ & $\begin{array}{l}0.425^{* *} \\
(0.173)\end{array}$ \\
\hline$(k / I)_{t-1}$ & $\begin{array}{c}0.479 * * \\
(0.208)\end{array}$ & $\begin{array}{c}0.243^{* *} \\
(0.107)\end{array}$ & $\begin{array}{l}0.142^{*} \\
(0.084)\end{array}$ & $\begin{array}{l}0.196 * * \\
(0.086)\end{array}$ & $\begin{array}{c}0.952 * * * \\
(0.360)\end{array}$ & $\begin{array}{l}0.623^{*} \\
(0.339)\end{array}$ & $\begin{array}{l}0.342^{*} \\
(0.185)\end{array}$ \\
\hline$\left(L_{r} / L\right)_{t-1}$ & $\begin{array}{l}2.158^{* *} \\
(0.941)\end{array}$ & $\begin{array}{l}2.512^{*} \\
(1.486)\end{array}$ & $\begin{array}{c}3.407 * * \\
(1.573)\end{array}$ & $\begin{array}{l}1.483 * * \\
(0.682)\end{array}$ & $\begin{array}{c}1.317^{* * *} \\
(0.411)\end{array}$ & $\begin{array}{l}1.160 * \\
(0.618)\end{array}$ & $\begin{array}{l}1.084 * \\
(0.630)\end{array}$ \\
\hline$\left(q / q_{l}\right)_{t-1}$ & $\begin{array}{c}-0.110 * * \\
(0.056)\end{array}$ & $\begin{array}{l}-0.265^{*} \\
(0.155)\end{array}$ & $\begin{array}{l}-0.697^{*} \\
(0.378)\end{array}$ & $\begin{array}{l}-0.107^{*} \\
(0.065)\end{array}$ & $\begin{array}{c}-0.441 * * \\
(0.225)\end{array}$ & $\begin{array}{c}-0.596 * * \\
(0.253)\end{array}$ & $\begin{array}{c}-0.286^{* *} \\
(0.113)\end{array}$ \\
\hline$\left(e / q_{l}\right)_{t-1}$ & $\begin{array}{c}-0.106 * * \\
(0.048)\end{array}$ & $\begin{array}{c}-0.754^{* *} \\
(0.380)\end{array}$ & $\begin{array}{l}-0.730 * \\
(0.443)\end{array}$ & $\begin{array}{l}-0.249 * \\
(0.147)\end{array}$ & $\begin{array}{l}-0.194 * \\
(0.118)\end{array}$ & $\begin{array}{c}-0.127^{* *} \\
(0.051)\end{array}$ & $\begin{array}{l}-0.453^{*} \\
(0.234)\end{array}$ \\
\hline OWN & $\begin{array}{l}-0.674 * \\
(0.357)\end{array}$ & $\begin{array}{l}-0.781 * \\
(0.408)\end{array}$ & $\begin{array}{c}-0.837 * * \\
(0.387)\end{array}$ & $\begin{array}{c}-1.382 * * \\
(0.659)\end{array}$ & $\begin{array}{c}-1.332 * * \\
(0.658)\end{array}$ & $\begin{array}{c}-1.411^{* * *} \\
(0.361)\end{array}$ & $\begin{array}{c}-0.786 * * \\
(0.358)\end{array}$ \\
\hline $\begin{array}{l}\text { Industry } \\
\text { dummies } \\
\text { indus1- } \\
\text { indus20 }\end{array}$ & included & included & included & included & included & included & included \\
\hline Intercept & $\begin{array}{c}-9.651 * * * \\
(3.327)\end{array}$ & $\begin{array}{c}-10.95 * * * \\
(3.434)\end{array}$ & $\begin{array}{c}-13.71 * * * \\
(5.083)\end{array}$ & $\begin{array}{c}-8.474 * * * \\
(2.360)\end{array}$ & $\begin{array}{c}-19.61 * * * \\
(6.431)\end{array}$ & $\begin{array}{c}-15.06 * * \\
(6.251)\end{array}$ & $\begin{array}{c}-9.946 * * * \\
(3.046)\end{array}$ \\
\hline Pseudo $\mathrm{R}^{2}$ & 0.233 & 0.267 & 0.273 & 0.233 & 0.355 & 0.239 & 0.183 \\
\hline $\begin{array}{l}\text { Log } \\
\text { likelihood }\end{array}$ & -36.00 & -26.72 & -24.69 & -34.51 & -16.40 & -30.74 & -32.84 \\
\hline $\begin{array}{l}\text { Number of } \\
\text { observations }\end{array}$ & 99 & 98 & 115 & 159 & 156 & 165 & 162 \\
\hline
\end{tabular}

Notes: $\quad * * *$ indicates significance at $1 \%, * *$ at $5 \%, *$ at $10 \%$. Standard errors in parentheses unless otherwise indicated. Lower case symbol denote the logarithm of the corresponding variable. $l: \log$ of number of employees, $k / l$ : capital intensity, $L_{r} / L$ : share of R\&D personnel, $q / q_{l}$ : market share, $e / q_{1}$ : export share, $O W N$ : origin country dummy ( Canada=1, Foreign=0).

In order to check for the success of the matching in removing differences between the group of treated firms (firms with tax credits and grants) and the group of non treated firms (firms with tax credits only), one need to check for the balance in the distribution of covariates between the two groups before and after performing matching. Matching is successful if no significant differences between the means of the two groups remain after its application. We first performed the widely used before-after mean comparison t-test in the evaluation literature. It consists in carrying out a t-test comparing the means of the covariates of the two groups before and after the matching to test whether the means are different.The results of this test before the matching and after the matching for the sample of all firms are presented in Table 6.B-2 and Table 6.B-3 respectively. Before the 
matching, for all covariates but the share of R\&D personnel, there is a significant difference between the two groups of means. After the matching, the differences in the means of the two groups in all covariates and in the propensity score are no longer significant. However, there are still differences in the outcome variable, R\&D intensity, as the coefficients in all periods are significant at the $5 \%$ level. These differences may be attributed to the receipt of public R\&D grants.

Then we computed, as suggested by Rosenbaum and Rubin (1985), the standardized difference statistic for the covariates of the two groups before and after the matching (results of test for all firms before and after matching in Table 6.B-2 and Table 6.B-3 respectively). This statistic is the difference of the means of the two groups as a percentage of the square root of the average of the sample variances of the two groups ${ }^{29}$. The results after the matching, show that standardized differences for all covariates, excluding the outcome variable, lie below the $10 \%$ threshold, that is, we therefore consider that the differences of the means of the two groups for all covariates are no longer significant. Indeed, though Rosenbaum and Rubin (1985) did not set a rule for the statistical significance of the standardized difference statistic, they considered absolute standardized differences below $10 \%$ as inconsequential. These results (for both the before-after mean comparison t-test and the standardized difference statistic) give us a great confidence in the quality of the matching. As an additional balancing test, we graphically compared the propensity scores of treated and non-treated groups before and after matching. As can be seen in Figure 6.C-1, the results confirm the success of the LLR matching estimator in removing differences between both groups.

We performed matching using PSMATCH2 Stata module by Leuven and Sianesi (2003). For each of the seven periods, we allowed matching only among treatment and control observations pertaining to the same industrial sector using NAICS 3 digit code. Given that the seven subsamples for each period are constructed from an unbalanced panel, certain firms are observed in more than one cross-section and, in several cases these firms switch from the status of treated (Tax credits $+R \& D$ grants) to the non-treated status (Tax credits only) from a given year to another and vice-versa. If we assume that the effect of treatment on a firm last more than one year, then a non-treated firm in a given year may not be a reliable match if it had the status of a treated firm in the previous year. For this reason and in order to ensure group homogeneity, we imposed an additional restriction on each

\footnotetext{
${ }^{29}$ Formally, the standardized difference statistic is a percent given by $100\left(\bar{x}_{1}-\bar{x}_{0 M}\right) /\left[\left(s_{1}^{2}+s_{0 R}^{2}\right) / 2\right]^{1 / 2}$, where, for each covariate, $\bar{x}_{1}$ and $\bar{x}_{0 M}$ are the sample means in the treated group and matched control group and $s_{1}^{2}$ and $s_{0 R}^{2}$ are the sample variances in the treated group and control reservoir (Rosenbaum and Rubin (1985)).
} 
comparison group constructed in the matching for the seven subsamples by limiting it only to firms that maintained a status of a non-treated firm in any year it is observed along the study period. Additionally, we imposed the common support region which consists in dropping treatment observations whose propensity score is higher than the maximum or less than the minimum propensity score of the observations in the control group. As a consequence, after the matching, the remaining observations in the three samples used are as follows: All firms, 7117 observations, 401 treated and 6716 non-treated; Low-medium technology sample, 6147 observations, 299 treated and 5848 non-treated; High technology, 876 observations, 99 treated and 876 non-treated. The weighting function used for the local linear regression matching is the triweight or tricube kernel $^{30}$ and the bandwidth is $0.08^{31}$.

Before turning to the findings from the application of the CDiD estimator, we should note that we also performed the CDiD using the Kernel matching estimator, but there were no much difference in the estimates reported. This must be caused by the fact that in our data there is substantial overlap in the distribution of the propensity score between the comparison and treatment groups. Dehejia and Wahba (2002) explain that in such case, most of the matching algorithms will yield similar results ${ }^{32}$.

The findings from the CDiD estimator for all firms and by technology level are reported in Table 6.5-5. Since weighted average outcomes for the non-treated firms enter the LLR matching estimator equation (see Section 6.3.1.2), the standard errors reported in the matching process for the CDiD coefficients are invalid. To deal with this issue, we bootstrapped these standard errors for all the CDiD estimates with 1000 replications. In sum, the results show an additionality effect of R\&D grants on firms' private investment on R\&D. More precisely, the average effect of treatment on R\&D intensity for the full sample varies between $2 \%$ and $11 \%$ from 1998 to 2004 . For the sub-sample of low-medium technology firms, which contains more than $86 \%$ of the observations of the full sample, the results are also quite similar. However, for the sub-sample of high technology firms, the CDiD estimates are a bit higher than the ones in the two previous cases, i.e. these estimates vary between $4 \%$ and $13 \%$. These results suggest the exclusion of possible crowding-out effects in a

\footnotetext{
${ }^{30}$ We also experimented with biweight (quartic) kernel, gaussian kernel and uniform kernel and it did not change significantly the results. A good review of weighting functions can be found in Li and Racine (2007).

${ }^{31}$ We also tested sensitivity of findings to various specifications on bandwidth but did not find significant differences.

${ }^{32}$ We find that the results from the LLR matching estimator exhibit significantly less bias error than the kernel-based matching estimator. Even though, differences in the estimates of the both matching estimators are not very important, the overall comparison shows that LLR matching is the best estimator for our data since it is more successful in removing differences between the group of firms with tax credits and grants and the group of firms with tax credits only.
} 
context where firms are given the choice of adding R\&D grants to R\&D tax credits. Furthermore, the results in the case of high technology firms suggest that in a fiscal environment such as that of Québec, high technology firms may be more responsive than other firms to direct public subsidies. Finally, as can be seen in these results, the magnitude of the response decreases between 1998 and 2004 in the three cases. Since the differences in the number of subsidized firms are not very important in the three samples, we attribute such a decline to the diminution in the value of grants observed in Québec between 1998 and 2004 (see Table 6.2-1).

Table 6.5-5: $\quad$ Estimated effect of treatment on R\&D intensity based on conditional difference-indifferences and local regression matching

\begin{tabular}{|c|c|c|c|}
\hline & All firms & $\begin{array}{l}\text { Low and medium- } \\
\text { technology }\end{array}$ & High technology \\
\hline \multirow[t]{2}{*}{1998} & $N_{1}=55, N_{0}=410$ & $N_{1}=37 N_{0}=375$ & $N_{1}=20, N_{0}=90$ \\
\hline & $\begin{array}{l}0.1122^{* * *} \\
(0.0002)\end{array}$ & $\begin{array}{l}0.1022^{* * *} \\
(0.0008)\end{array}$ & $\begin{array}{l}0.1269 * * * \\
(0.0021)\end{array}$ \\
\hline \multirow[t]{2}{*}{1999} & $N_{1}=56, N_{0}=477$ & $\mathrm{~N}_{1}=40 \mathrm{~N}_{0}=413$ & $N_{1}=14, N_{0}=98$ \\
\hline & $\begin{array}{l}0.1108^{* * *} \\
(0.0002)\end{array}$ & $\begin{array}{l}0.1001 * * * \\
(0.0007)\end{array}$ & $\begin{array}{l}0.1248^{* * *} \\
(0.0021)\end{array}$ \\
\hline \multirow[t]{2}{*}{2000} & $N_{1}=73, N_{0}=1063$ & $N_{1}=58 N_{0}=933$ & $N_{1}=15, N_{0}=112$ \\
\hline & $\begin{array}{l}0.0996 * * * \\
(0.0005)\end{array}$ & $\begin{array}{l}0.0979 * * * \\
(0.0008)\end{array}$ & $\begin{array}{l}0.1067 * * * \\
(0.0037)\end{array}$ \\
\hline \multirow[t]{2}{*}{2001} & $\mathrm{~N}_{1}=55, \mathrm{~N}_{0}=1014$ & $\mathrm{~N}_{1}=40, \mathrm{~N}_{0}=899$ & $\mathrm{~N}_{1}=15, \mathrm{~N}_{0}=120$ \\
\hline & $\begin{array}{l}0.0503^{* * *} \\
(0.0001)\end{array}$ & $\begin{array}{l}0.0407^{* * *} \\
(0.0003)\end{array}$ & $\begin{array}{l}0.0528 * * * \\
(0.0031)\end{array}$ \\
\hline \multirow[t]{2}{*}{2002} & $N_{1}=58, N_{0}=1270$ & $N_{1}=46, N_{0}=1033$ & $N_{1}=10, N_{0}=118$ \\
\hline & $\begin{array}{l}0.0500 * * * \\
(0.0002)\end{array}$ & $\begin{array}{l}0.0399 * * * \\
(0.0005)\end{array}$ & $\begin{array}{l}0.0511^{* * *} \\
(0.0036)\end{array}$ \\
\hline \multirow[t]{2}{*}{2003} & $\mathrm{~N}_{1}=53, \mathrm{~N}_{0}=1302$ & $\mathrm{~N}_{1}=40, \mathrm{~N}_{0}=1116$ & $N_{1}=12, N_{0}=122$ \\
\hline & $\begin{array}{l}0.0243 \\
(0.0013)\end{array}$ & $\begin{array}{l}0.0201 \\
(0.0020)\end{array}$ & $\begin{array}{l}0.0389 * * * \\
(0.0026)\end{array}$ \\
\hline \multirow[t]{3}{*}{2004} & $N_{1}=51, N_{0}=1180$ & $N_{1}=38 N_{0}=1079$ & $N_{1}=13, N_{0}=117$ \\
\hline & 0.0245 & 0.0233 & $0.0392 * * *$ \\
\hline & $(0.0010)$ & $(0.0017)$ & $(0.0026)$ \\
\hline
\end{tabular}

Note: $\quad * * *$ indicates significance at $1 \%, * *$ at $5 \%, *$ at $10 \%$. Bootstrap standard errors in parentheses with 1000 replications. Bandwidth: 0.08 . 


\subsubsection{Assessing the Impact of Privately and Publicly Funded R\&D on Productivity Growth}

We estimated Equation (6-15) described in Section 6.3.2. For the two components of R\&D intensity that enter that equation i.e., the R\&D intensity induced by $R \& D$ expenditures funded by $R \& D$ grants $(R " / Q)$ and the $R \& D$ intensity induced by private $R \& D$ expenditures and by tax credits $\left(R^{\prime} / Q\right)$, we take the two variables estimated in the previous section on matching, respectively $R " / Q=\tau_{D i D}$ and $R^{\prime} / Q=Y^{0}=Y^{1}-\tau_{D i D}$. However, these variables should not enter the equation as contemporary variables since the effect of a shock on R\&D intensity is likely to take a certain time before it is fully reflected in firm's productivity growth. It is often argued that a lag structure of several periods should be included in order to account for the adjustment of productivity growth. According to the Bureau of Labor Statistics (1989) survey of the literature relating R\&D to productivity growth, the productivity gains should lag the R\&D outlays by at least one year. Hence, we therefore included one-period lagged values of $R^{\prime \prime} / Q$ and $R^{\prime} / Q$ in the regression due to relatively small number of observations in the subsample of treated firms.

We used value added as proxy for firm's output $Q$ and we corrected our data to prevent bias in the estimates that can arise from double counting as warned by Hall and Mairesse (1995). To do so, we respectively subtracted R\&D capital ${ }^{33}$ from the assets and R\&D personnel from the number of employees in the production function and, added the materials component of R\&D expenditure into value added. Value added, the capital stock and R\&D expenditures have been deflated respectively by the industry output price deflator at the 3-digit NAICS code, the machinery and equipment price indexes and by the Jaffe-Griliches R\&D deflator (see Bureau of Labor Statistics (1989) for the latter).

For the estimation procedure, we first performed OLS regression of Equation (6-15) with and without time and industry dummies. We also performed the Newey-West two-step efficient GMM estimation of this equation to allow for the fact that R\&D intensity components ( $R^{\prime \prime} / Q$ and $R^{\prime} / Q$ ) may be endogenous, i.e. the fact that R\&D intensity and productivity may be mutually dependent. Indeed, when this hypothesis is verified, OLS estimates are biased because the assumption requiring that explanatory variables be independent is no longer valid. To correct for this problem, several statistical techniques such as indirect least squares, instrumental variable and two- and three-stage least squares may be applied. Hence, to verify whether R\&D intensity components are endogenous, we ran the $C$ or "difference-in-Sargan" test and for the two components, the test rejected the null that they are not endogenous regressors in the equations for the two-step

\footnotetext{
${ }^{33}$ For the details concerning the construction of this variable, see Baghana and Mohnen (2009).
} 
GMM estimator. This confirmed the need to instrument both $R^{\prime \prime} / Q$ and $R^{\prime} / Q$. To this end, we experimented with several sets of variables and we ended-up using the following set: the second and third lagged values of R\&D intensity, the lagged value of the share of R\&D personnel and lagged value of the sum of provincial and federal grants. Table 6.5-6 shows the estimation results of Equation (6-15) estimated by OLS and GMM respectively. It should be noted that, for comparison purposes, since the estimation by GMM is Heteroscedasticity-consistent (HC), we estimated the OLS using the Huber-White sandwich estimator of variance, which is Heteroscedasticityconsistent, in place of the traditional calculation. For each case we ran the estimation with and without the dummies for time and for industry. For this last part of the analysis, only the larger sample of all firms is considered. We excluded the sub-samples of low-medium and high technology firms due to the important reduction in the number of observation in the group of treated firms after differencing.

Comparing the estimated models, GMM estimated model 4 is clearly superior and its relative lower AIC value (14 513.86) among the estimated models adds to this evidence. Furthermore, model 4 is the one having the best fit since all the parameters of interest (i.e. the coefficient in the two R\&D intensity components, in capital labour growth rate and in employment growth rate) are significant at the $10 \%$ level of confidence. We therefore selected model 4 as our preferred model.

We ran several additional tests in order to check for the robustness of the specification of the selected model. First, as time and industry dummy variables were individually insignificant based on t-tests with very high $p$ values, we proceeded with testing for the significance of a subset of coefficients using the Wald test. We could not reject the null that industry dummies, in the one hand, and time dummies, in the other hand, are jointly insignificant. In consequence, these dummies were excluded from Model 4.

To check the relevance of our excluded instruments, we performed the Kleibergen and Paap (2006) rank LM statistic test of underidentification which is robust to heteroskedasticity, autocorrelation or clustering. The reported statistic of 24.46 falls within the region of rejection as the $p$-value $(0.000)$ strongly reject the null that the equation is underidentified at the $1 \%$ significance level. We further proceeded with testing for the presence of weak instruments since it is well known that even if the equation is identified, there may still be weak instrument problems often responsible of poor performance of estimators. We further proceeded with testing for the presence of weak instruments using the Cragg-Donald F statistic and critical values tabulated by Stock and Yogo (2005). At the $5 \%$ significance level, the test rejected the null hypothesis that the instruments are weak in both alternative definitions offered by Stock and Yogo (2005) i.e. first, the bias of the IV estimator, relative to the bias of the OLS, could exceed the threshold of $30 \%$ and, second, the bias in the size of the Wald test based in the IV statistics could exceed $25 \%$. Finally, 
with a statistic value of 6.33 , we fail to reject the Hansen J overidentification test, giving us greater confidence that our instrument set is relevant.

Table 6.5-6: Estimation results of OLS-HC and GMM models for the productivity growth, all firms

\begin{tabular}{|c|c|c|c|c|}
\hline \multirow[t]{2}{*}{ Parameters/Statistics } & \multicolumn{2}{|c|}{ OLS-HC } & \multicolumn{2}{|c|}{ GMM } \\
\hline & (1) & (2) & (3) & (4) \\
\hline \multicolumn{5}{|l|}{$\begin{array}{l}\text { Dependent variable: } \\
\Delta(q / I)_{t}\end{array}$} \\
\hline$\left(R^{\prime \prime} / Q\right)_{t-1}$ & $\begin{array}{c}-0.154^{* * *} \\
(0.038)\end{array}$ & $\begin{array}{c}-0.156 * * * \\
(0.038)\end{array}$ & $\begin{array}{c}0.367^{* * *} \\
(0.097)\end{array}$ & $\begin{array}{l}0.134^{*} \\
(0.075)\end{array}$ \\
\hline$\left(R^{\prime} / Q\right)_{t-1}$ & $\begin{array}{c}-0.0387^{*} \\
(0.023)\end{array}$ & $\begin{array}{c}-0.0406^{*} \\
(0.023)\end{array}$ & $\begin{array}{c}0.426 * * * \\
(0.108)\end{array}$ & $\begin{array}{c}0.322 * * * \\
(0.048)\end{array}$ \\
\hline$\Delta(k / I)_{t}$ & $\begin{array}{c}0.112 * * * \\
(0.014)\end{array}$ & $\begin{array}{c}0.110 * * * \\
(0.014)\end{array}$ & $\begin{array}{c}0.0385^{* *} \\
(0.018)\end{array}$ & $\begin{array}{c}0.0971 * * * \\
(0.013)\end{array}$ \\
\hline$\Delta I_{t}$ & $\begin{array}{c}-0.198^{* * *} \\
(0.019)\end{array}$ & $\begin{array}{c}-0.200 * * * \\
(0.019)\end{array}$ & $\begin{array}{c}-0.0616 * * * \\
(0.022)\end{array}$ & $\begin{array}{c}-0.190 * * * \\
(0.014)\end{array}$ \\
\hline $\begin{array}{l}\text { Year dummies } \\
t 1-t 7\end{array}$ & Not included & Included & Included & Not included \\
\hline $\begin{array}{l}\text { Industries dummies } \\
\text { Indus 1-Indus } 20\end{array}$ & Not included & Included & Included & Not included \\
\hline Intercept & $\begin{array}{c}0.181 \\
(0.145)\end{array}$ & $\begin{array}{c}0.263^{* * *} \\
(0.031)\end{array}$ & $\begin{array}{l}0.0667 \\
(0.068)\end{array}$ & $\begin{array}{c}0.221 * * * \\
(0.032)\end{array}$ \\
\hline $\begin{array}{l}\text { F test } \\
\text { (p-value) }\end{array}$ & $\begin{array}{c}6.61 \\
(0.000)\end{array}$ & $\begin{array}{c}34.49 \\
(0.000)\end{array}$ & $\begin{array}{c}2.16 \\
(0.001)\end{array}$ & $\begin{array}{l}124.32 \\
(0.000)\end{array}$ \\
\hline Log likelihood & -8315.73 & -8337.55 & -8432.84 & -7251.93 \\
\hline $\mathrm{AIC}$ & 16687.45 & 16685.10 & 16911.68 & 14513.86 \\
\hline $\begin{array}{l}\text { Test of } \\
\text { underidentification: } \\
\text { Kleibergen-Paap rank LM } \\
\text { statistic } \\
\text { (p-value) }\end{array}$ & & & $\begin{array}{c}23.92 \\
(0.000)\end{array}$ & $\begin{array}{c}24.46 \\
(0.000)\end{array}$ \\
\hline $\begin{array}{l}\text { Test of weak } \\
\text { identification: }\end{array}$ & & & & \\
\hline $\begin{array}{l}\text { Cragg-Donald F statistic } \\
\text { (at } 5 \% \text { level of } \\
\text { confidence) }\end{array}$ & & & 5.98 & 6.33 \\
\hline $\begin{array}{l}\text { - IV relative bias to } \\
\text { OLS }\end{array}$ & & & $\begin{array}{l}\text { Rejected } \\
>30 \%\end{array}$ & $\begin{array}{l}\text { Rejected } \\
>20 \%\end{array}$ \\
\hline — Size bias & & & $\begin{array}{l}\text { Not rejected } \\
\quad>25 \%\end{array}$ & $\begin{array}{c}\text { Rejected } \\
>25 \%\end{array}$ \\
\hline $\begin{array}{l}\text { Test of } \\
\text { overidentification: } \\
\text { Hansen J-statistic } \\
\text { (p-value) }\end{array}$ & & & $\begin{array}{c}3.99 \\
(0.136)\end{array}$ & $\begin{array}{c}4.10 \\
(0.129)\end{array}$ \\
\hline Number of observations & 7406 & 7406 & 6071 & 6316 \\
\hline
\end{tabular}

Notes: Estimation period is $1998-2004{ }^{* * *}$ indicates significance at $1 \%,{ }^{* *}$ at $5 \%, *$ at $10 \%$. Standard errors in parentheses unless otherwise indicated. Lower case symbol denote the logarithm of the corresponding variable. $\Delta(q / I)$ : labour productivity growth rate; $R^{\prime} / Q: R \& D$ intensity (induced by private R\&D expenditures and by tax credits); $R " / Q: R \& D$ intensity induced by $R \& D$ expenditures funded by public grants; $\Delta(k / l)$ : capital-labour growth rate; $\Delta l$ : employment growth rate. 
Regarding the significance of the coefficients, as can be seen, all the coefficients in our preferred econometric model (Model 4) are significant at the $1 \%$ level with an exception for the coefficient related to the variable of R\&D expenditures funded by public grants which is significant at the $10 \%$ level. Furthermore, except for the coefficient of employment growth that have a negative sign, all the coefficients have a positive sign, suggesting a positive effect on labour productivity growth rate. The result for the estimated coefficient for the capital-labour growth rate shows a statistically significant role and amounts to 0.097 , which is quite close to the results reported in the literature. The estimated coefficient for the employment growth rate exhibits a negative sign and the t-test strongly rejects the null hypothesis that it is equal to zero. As pointed in Section 6.3.2, this means that constant return to scale is rejected and that firms are facing decreasing return to scale in Québec. Similar results have been reported by Griliches and Mairesse (1991) for US and Japan, Wakelin (2001) for UK, Smith et al. (2004) for Denmark and Maté-Garcia and Rodriguez-Fernandez (2008) for Spain.

The rates of return to $R \& D$ expenditures funded by public grants and to $R \& D$ expenditures privately funded and by tax credits are respectively 0.134 and 0.322 . This means that for each additional dollar of public R\&D grant, output increases by 0.134 dollars in the former case and that, for each additional dollar of the remaining $R \& D$ expenditures (funded privately and by tax credits), output increases by 0.322 dollars. It is noteworthy that, although these positive results support direct subsidies programs, they also show that the additional return of direct subsidies is positive but lower than the return on the R\&D financed by own funds or R\&D tax credits. From a comparative point of view with the empirical literature, the estimated rates of return of 0.134 and 0.322 are consistent with most studies since they fall within the 0.2-0.5 interval of the values estimated by these studies. We should note however that this comparison must be taken with caution since these studies don't break R\&D spending in various components as in the case of this chapter. The ratio of both rates of return is of 0.42 which indicates that public R\&D grants represent $42 \%$ of the productivity induced by both private funds and R\&D tax credits.

\subsection{Conclusion}

Investigating the effectiveness of public grants as a significant driver in firms' private spending for $R \& D$ and the impact of the latter on productivity, have become important policy issues in a context where fiscal incentives are present. Using longitudinal data through the period 1997 to 2004 from manufacturing firms in Québec, we analyse these issues in two steps: In the first step, we assess the input additionality of public R\&D grants in terms of increased R\&D spending from firms 
that already receive tax credits and, in the second step, in terms of productivity growth. As far we know, this is the first empirical analysis that integrates these issues in one analysis framework by using micro data from Québec.

Our results show that firms that use public grants for R\&D in conjunction with tax credits for R\&D perform better in terms of R\&D input additionality and in terms of growth productivity than firms that use only tax credits for R\&D. In particular, we find in the first step that the R\&D intensity of firms in the former group is higher than that of firms in the latter group. Our results also support the hypothesis that high-tech firms benefit the most from public grants financing. Indeed, we find that the magnitude of the impact of $R \& D$ grants in the intensity of $R \& D$ of high-tech firms is greater than that of low and medium technology firms.

In the second step, the first main result is that the rate of return to $R \& D$ expenditures funded by public R\&D grants is of 0.134 i.e. for each additional dollar of public R\&D grant, output increase by 0.134 dollars. This finding permits to conclude that the additional return of direct subsidies is positive but lower than the return on the R\&D financed by own funds or $R \& D$ tax credits. The second main result is that public $R \& D$ grants represent $42 \%$ of the productivity induced by both private funds and R\&D tax credits. These high ratios provide support to the use of public R\&D grants as additional policy instrument to tax credits. In other words, since $R \& D$ grants and $R \& D$ tax credits work well together, the question for policy makers is less whether to choose between R\&D grants and R\&D tax credits. The question is rather in terms of the capacity of public agency responsible of the attribution of R\&D grants to identify the projects for which R\&D tax credits fail to offer an incentive for the private projects and to find a suitable level of additional funding in a context where firms already benefit from fiscal incentives. 


\section{Chapter Appendices}

\section{A. Description of Variables}

Table 6.A-1: Variables construction and descriptive statistics

\begin{tabular}{|c|c|c|c|}
\hline Variable & Construction & Mean & S.D. \\
\hline$L$ & Firm's number of employees & 111.09 & 747.65 \\
\hline I & Firm's number of employees, in logarithm & 3.05 & 1.61 \\
\hline$K$ & Tangible assets in real terms, in million 2002 CAN\$ & 40.00 & 540.00 \\
\hline$k / I$ & $\begin{array}{l}\text { Capital intensity, constructed as firm's real assets divided by } \\
\text { the number of employees, in logarithm. Firm's real assets is } \\
\text { deflated by the machinery and equipment price indexes }\end{array}$ & 11.74 & 1.17 \\
\hline$I_{r} / I$ & $\begin{array}{l}\text { Share of R\&D personnel, constructed as firm's R\&D } \\
\text { personnel divided by the number of employees }\end{array}$ & 0.19 & 0.27 \\
\hline$q$ & $\begin{array}{l}\text { Firm's value added in real terms, in logarithm, deflated by } \\
\text { the industry output price deflator at the 3-digit NAICS code }\end{array}$ & 15.09 & 1.78 \\
\hline$q_{1}$ & $\begin{array}{l}\text { Industry sales in real terms, } \\
\text { (at 3-digit NAICS classification), in logarithm }\end{array}$ & 21.74 & 0.60 \\
\hline$q / q_{1}$ & $\begin{array}{l}\text { Market share, constructed as firm's real sales divided by } \\
\text { industry's real sales, in logarithm }\end{array}$ & -6.65 & 1.85 \\
\hline$e$ & Firm exportations in real terms, in logarithm & 14.94 & 1.72 \\
\hline$e / q_{l}$ & $\begin{array}{l}\text { Export share, constructed as firm's real exportations divided } \\
\text { by industry's real sales, in logarithm }\end{array}$ & -0.13 & 0.57 \\
\hline$R$ & R\&D expenditures, in million 2002 CAN\$ & 0.59 & 6.8 \\
\hline$R / Q$ & $\begin{array}{l}\text { R\&D intensity, constructed as firm's real R\&D expenditures } \\
\text { divided by firm's real value added. Firm's real R\&D is } \\
\text { deflated by the R\&D deflator }\end{array}$ & 0.06 & 0.11 \\
\hline $\begin{array}{l}\text { Year dummies } \\
t 1-t 7\end{array}$ & t1-t7 for years 1998, 1999, 2000, 2001, 2002, 2003 and 2004 & & \\
\hline $\begin{array}{l}\text { Industry dummies } \\
\text { indus1-indus } 20\end{array}$ & $\begin{array}{l}20 \text { industry dummies for industries at NAICS } 3 \text { digit code } \\
\text { level: } 311,312,313,314,315,316,321,322,323,324,325 \text {, } \\
326,327,331,332,333,334,335,336,337\end{array}$ & & \\
\hline$q / /$ & $\begin{array}{l}\text { Labour productivity, constructed as firm's real value added } \\
\text { divided by the number of employees, in logarithm. }\end{array}$ & 12.03 & 1.17 \\
\hline$\Delta(q / I)$ & $\begin{array}{l}\text { Labour productivity growth rate, constructed as the } \\
\text { logarithm of the change of the ratio of firm's real value } \\
\text { added to the number of employees }\end{array}$ & 0.02 & 0.80 \\
\hline$\Delta(k / I)$ & $\begin{array}{l}\text { Capital-labour growth rate, constructed as the logarithm of } \\
\text { the change of the ratio of firm's real capital to the number of } \\
\text { employees }\end{array}$ & 0.05 & 0.78 \\
\hline$\Delta l$ & $\begin{array}{l}\text { Employment growth rate, constructed as the logarithm of } \\
\text { the change of the number of employees }\end{array}$ & 0.06 & 0.69 \\
\hline
\end{tabular}

Note: Lower case symbol denote the logarithm of the corresponding variable. The descriptive statistics are sample means for the years 1997-2004. The base year is 2002. Number of observations: 11842 (3815 firms). 


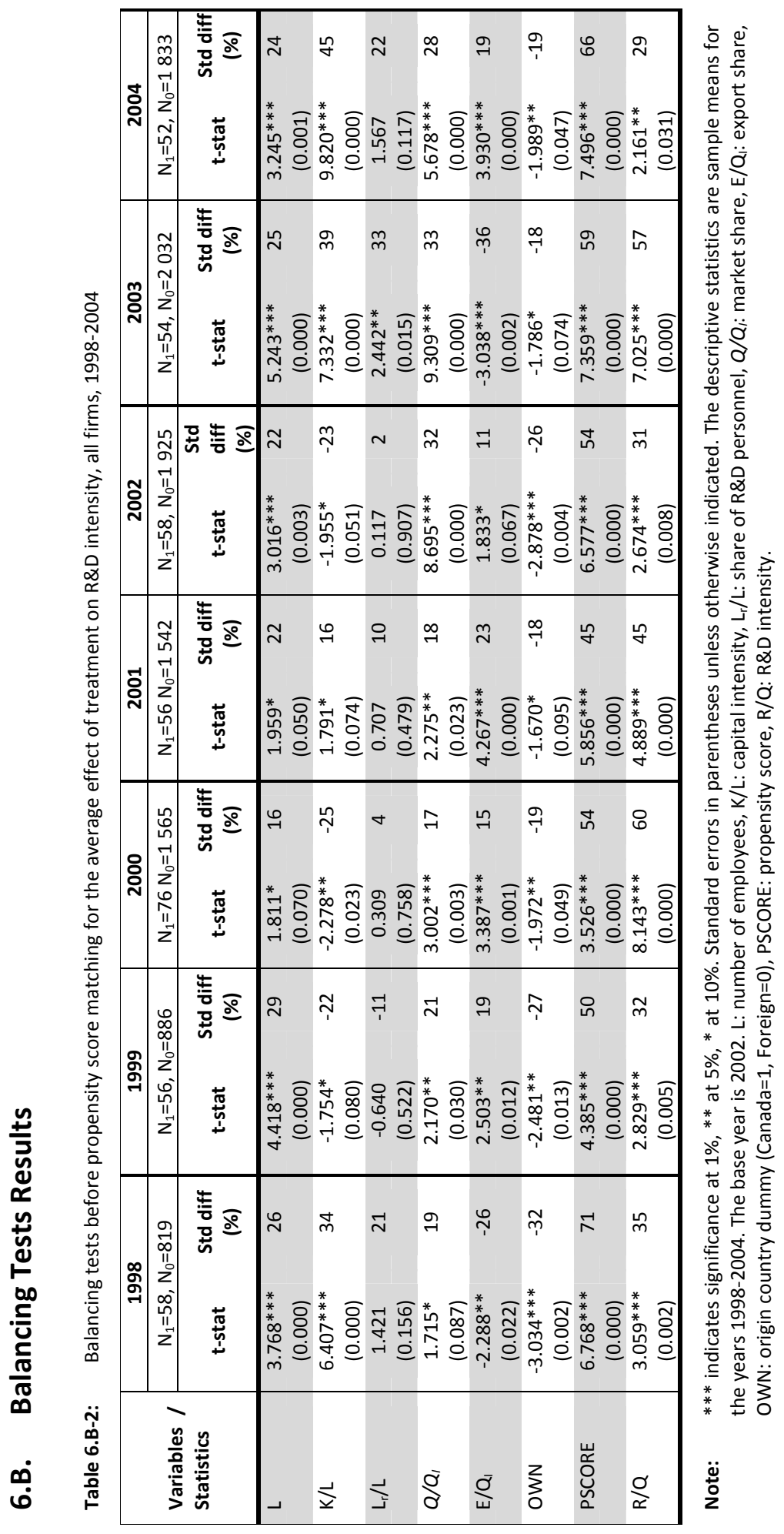




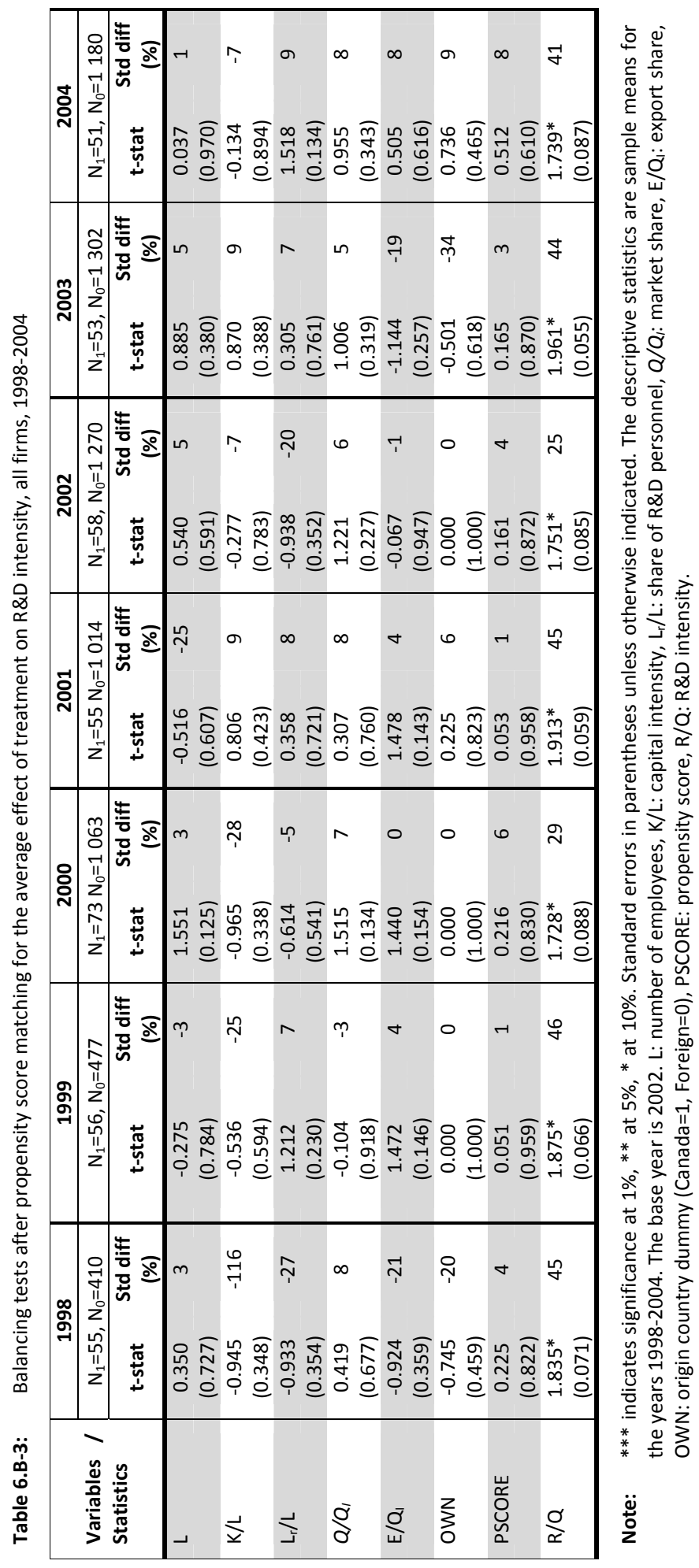




\section{C. Distribution of Propensity Score}

Figure 6.C-1: Distribution of propensity score before and after matching, all firms, 1998-2004

Distribution of propensity score before and after matching - 1998-2004, All firms

1998

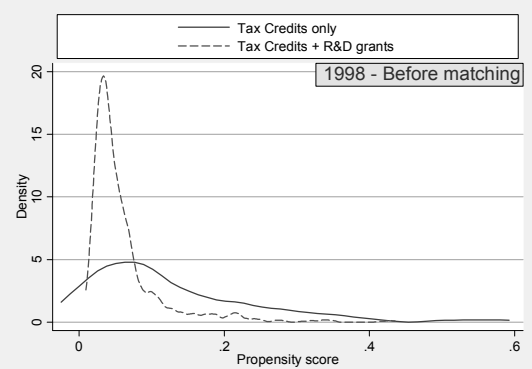

1999

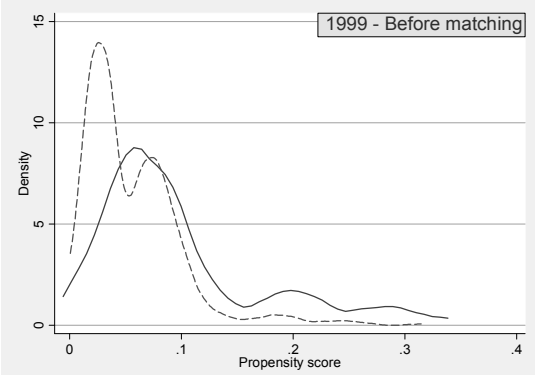

2000

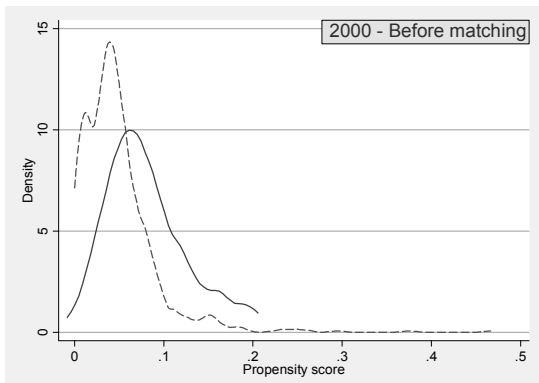

2001 - Before matching

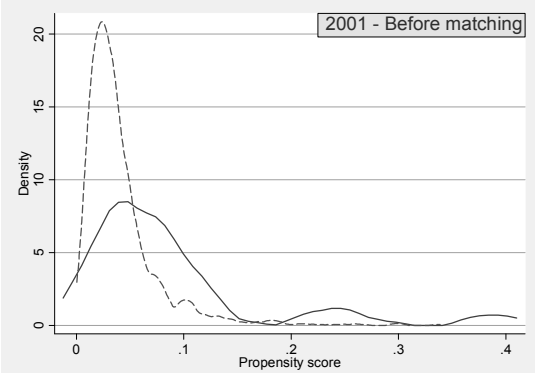

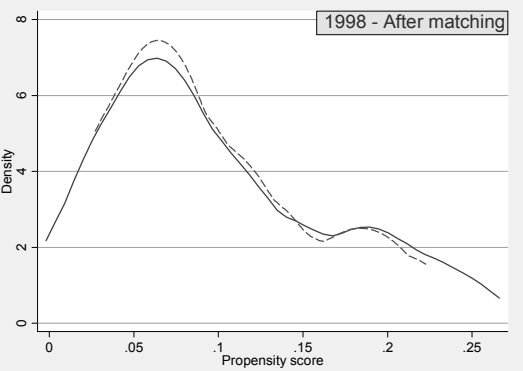
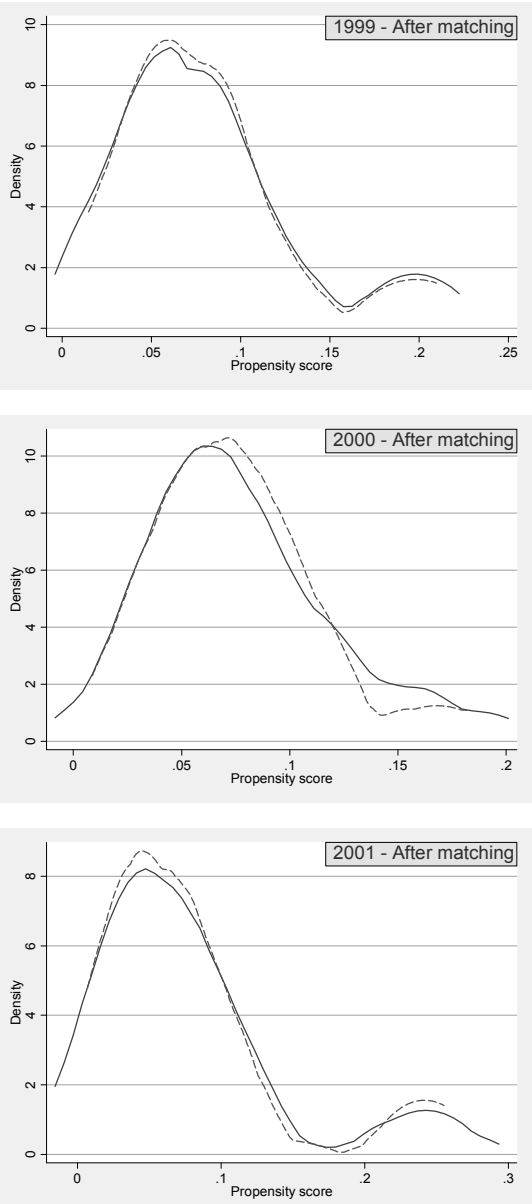
Distribution of propensity score before and after matching - 1998-2004, All firms

2002
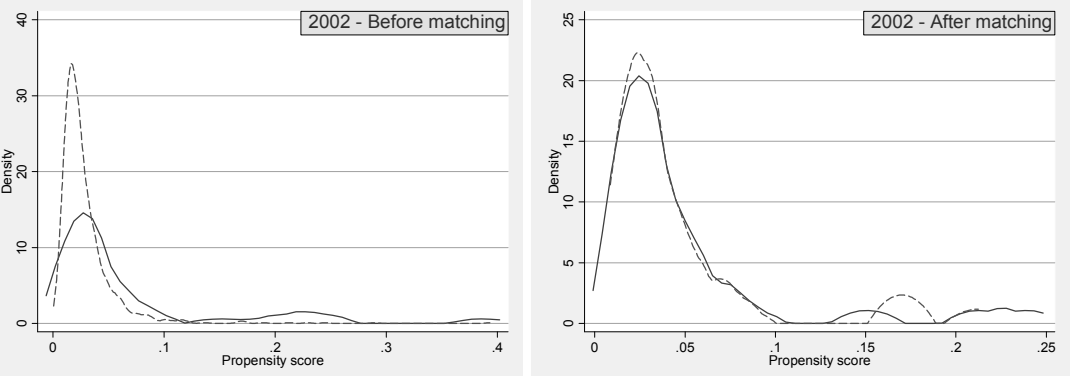

2003
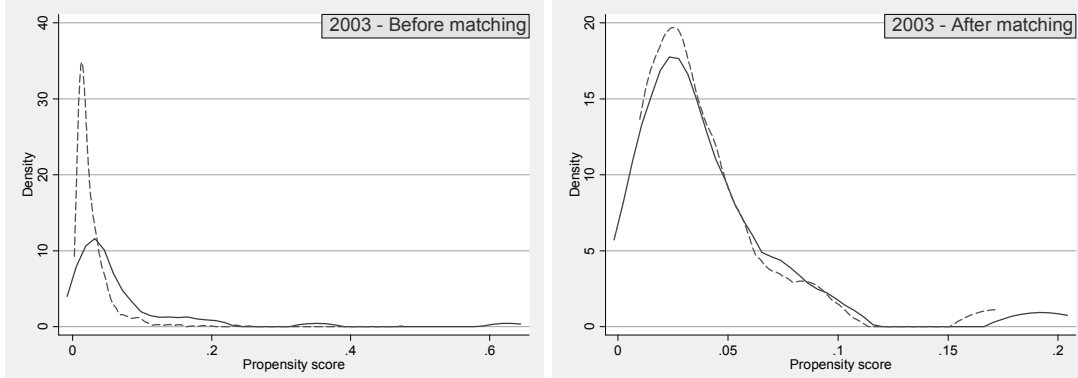

2004
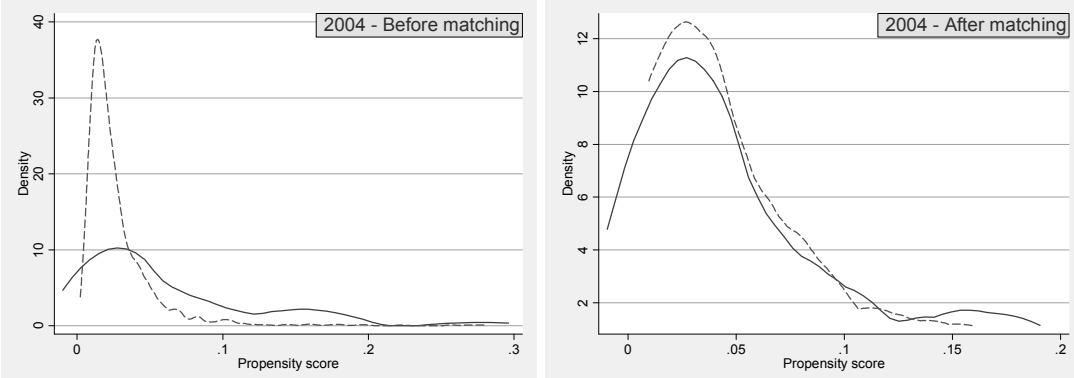


\section{D. Classification of Manufacturing Industries}

Table 6.D-4: OECD Classification of manufacturing industries based on technology using ISIC rev.3 activity breakdown

ISIC rev.3 code

$1 \quad$ High-technology industries

Aircraft and spacecraft

Pharmaceuticals

Office, accounting and computing machinery

Radio, TV and communciations equipment

Medical, precision and optical instruments

2 Medium-high-technology industries

Electrical machinery and apparatus, n.e.c

Motor vehicles, trailers and semi-trailers

Chemicals excluding pharmaceuticals

Railroad equipment and transport equipment, n.e.c.

Machinery and equipment, n.e.c.

3 Medium-low-technology industries

Building and repairing of ships and boats

Rubber and plastics products

Coke, refined petroleum products and nuclear fuel

Other non-metallic mineral products

Basic metals and fabricated metal products

$4 \quad$ Low-technology industries

Manufacturing, n.e.c.; Recycling

Wood, pulp, paper, paper products, printing and publishing

Textiles, textile products, leather and footwear

Source: OECD, 2005.

Note: ISIC: International Standard Industrial Classification. 
Table 6.D-5: Classification of manufacturing industries based on the type of activity using NAICS 3 digit code breakdown

\begin{tabular}{llc}
\hline Dummy & & $\begin{array}{c}\text { NAICS 3 digit } \\
\text { code }\end{array}$ \\
\hline Indus 01 & Food Manufacturing & 311 \\
\hline Indus 02 & Beverage and Tobacco Product Manufacturing & 312 \\
\hline Indus 03 & Textile Mills & 313 \\
\hline Indus 04 & Textile Product Mills & 314 \\
\hline Indus 05 & Clothing Manufacturing & 315 \\
\hline Indus 06 & Leather and Allied Product Manufacturing & 316 \\
\hline Indus 07 & Wood Product Manufacturing & 321 \\
\hline Indus 08 & Paper Manufacturing & 322 \\
\hline Indus 09 & Printing and Related Support Activities & 323 \\
\hline Indus 10 & Petroleum and Coal Products Manufacturing & 324 \\
\hline Indus 11 & Chemical Manufacturing & 325 \\
\hline Indus 12 & Plastics and Rubber Products Manufacturing & 326 \\
\hline Indus 13 & Non-Metallic Mineral Product Manufacturing & 327 \\
\hline Indus 14 & Primary Metal Manufacturing & 331 \\
\hline Indus 15 & Fabricated Metal Product Manufacturing & 332 \\
\hline Indus 16 & Machinery Manufacturing & 333 \\
\hline Indus 17 & Computer and Electronic Product Manufacturing & 334 \\
\hline Indus 18 & Electrical Equipment, Appliance and Component Manufacturing & 335 \\
\hline Indus 19 & Transportation Equipment Manufacturing & 336 \\
\hline Indus 20 & Furniture and Related Product Manufacturing & 337 \\
\hline Indus 21 & Miscellaneous Manufacturing & 339 \\
\hline
\end{tabular}

Source: Statistics Canada.

Note: NAICS: North American Industry Classification System. 


\section{E. Derivation of the Labour Productivity Equation}

Consider a typical a Cobb-Douglas production function given by:

$Q_{i t}=A e^{\lambda_{t}} K_{i t}^{\alpha} L_{i t}^{\beta} S_{i t}^{\prime \gamma^{\prime}} S^{\prime \prime} \gamma_{i t}^{\prime \prime} e^{\varepsilon_{i t}}$

where:

$i=$ firms;

$\mathrm{t}=$ years;

$Q=$ output;

$A=$ constant;

$\lambda=$ scale factor measuring the rate of disembodied technical change;

$\alpha=$ elasticity of output with respect to physical capital;

$\beta=$ elasticity of output with respect to labour;

$\gamma^{\prime}=$ elasticity of output with respect to knowledge capital induced by private R\&D and by tax credits;

$\gamma^{\prime}=$ the elasticity of output with respect to knowledge capital induced by public grants;

$K=$ physical capital;

$L=$ labour;

$S^{\prime}=$ share of knowledge capital induced by R\&D expenditures funded privately and by tax credits;

$S^{\prime \prime}=$ share of knowledge capital induced by R\&D expenditures funded by public grants;

$\varepsilon=$ error term.

If we take the logarithm and subtract both sides of Equation (6-16) by the logarithm of labour, we get:

$q_{i t}-l_{i t}=a+\lambda t+\alpha k_{i t}+\beta l_{i t}+\gamma^{\prime} s^{\prime}{ }_{i t}+\gamma^{\prime \prime} s^{\prime \prime}{ }_{i t}+l_{i t}+\varepsilon_{i t}$

By assuming that the constant returns to scale coefficient is given by $\theta=\alpha+\beta-1$, this measure should equal zero in the case of constant return to scale. However, if this measure is less than zero or greater than zero, then increasing return to scale and decreasing return to scale will be assumed respectively. If we substitute $\beta=\theta+1-\alpha$ into Equation (6-17) and re-order terms, we get the following expression:

$q_{i t}-l_{i t}=a+\lambda t+\alpha(k-l)_{i t}+\theta l_{i t}+\gamma^{\prime} s^{\prime}{ }_{i t}+\gamma^{\prime \prime} s^{\prime \prime}{ }_{i t}+\varepsilon_{i t}$ 
First-differencing ( 6-18) yields the following:

$$
\Delta(q-l)_{i t}=\lambda+\alpha \Delta(k-l)_{i t}+\theta \Delta I_{i t}+\gamma^{\prime} \Delta s^{\prime}{ }_{i t}+\gamma^{\prime \prime} \Delta s^{\prime \prime}{ }_{i t}+\varepsilon_{i t}
$$

Since, in the first step, we used the capital intensity as a proxy for the knowledge capital, we need to transform Equation (6-19) in a way such that it includes capital intensity in its functional form instead of knowledge capital. This is done by using the usual relation linking the output elasticity with regard to knowledge capital to the rate of return, which is:

$$
\gamma=\rho\left(\frac{S}{Q}\right)_{i t}
$$

where $\rho$ is the rate of return, and by assuming that the growth rate of the knowledge capital is given by:

$$
\Delta s_{i t}=\left(\frac{\partial S}{S}\right)_{i t}
$$

Equations (6-20) and (6-21) give:

$$
\gamma \Delta s_{i t}=\rho\left(\frac{S}{Q}\right)_{i t}\left(\frac{\partial S}{S}\right)_{i t}
$$

The change in the knowledge capital $\partial S$ may be approximated by current R\&D expenditures $R$ if we assume that there is no depreciation in the knowledge capital. Then we can write that $\gamma^{\prime} \Delta s^{\prime}{ }_{i t}=\rho^{\prime}\left(\frac{R^{\prime}}{Q}\right)_{i t}$ and $\gamma^{\prime \prime} \Delta s^{\prime \prime}{ }_{i t}=\rho^{\prime}\left(\frac{R^{\prime \prime}}{Q}\right)_{i t}$, and by substituting these expressions into Equation (6-19) we get the labour productivity growth as follows:

$$
\Delta\left(q_{i t}-l_{i t}\right)=\lambda+\alpha \Delta(k-l)_{i t}+\theta \Delta I_{i t}+\rho^{\prime}\left(\frac{R^{\prime}}{Q}\right)_{i t}+\rho^{\prime \prime}\left(\frac{R^{\prime \prime}}{Q}\right)_{i t}+v_{i t}
$$

It is worth mentioning that Equation (6-23) in which the main parameter of interest is the rate of return $\rho$ has been widely used in the literature as an alternative to Equation (6-19) in which the parameter of interest is instead the elasticity $\gamma$. This has been particularly the case because of the lack of data to construct the knowledge capital variable. 


\section{CHAPTER 7}

\section{Is Private R\&D Spending \\ Sensitive to Increments in Fiscal \\ incentives? Evidence from the}

Superdeductions Program in

Québec

A previous version of this chapter has been published as:

Baghana, Rufin 2008, Une évaluation de l'impact du programme québécois de superdéductions à la $R-D$, in Institut de la Statistique du Québec, ed.: Compendium d'indicateurs de l'activité scientifique et technologique au Québec. Édition 2008 (Institut de la Statistique du Québec, Québec). 


\subsection{Introduction}

Introduced in Québec during the Budget Speech in March 1999, the superdeductions for R\&D were a tax measure that entitled an additional deduction to firms performing R\&D activities in Québec in the computation of their income in addition to the usual deduction of total current expenditure of R\&D. Theoretically, superdeductions were supposed to provide companies with a tax benefit equal to that of the SR\&ED tax credits since their rates were calibrated according to the rates of the SR\&ED tax credits and tax rates on company profits. In reality, the lower cost of $R \& D$ arising from superdeductions was significantly greater than that which flowed from the SR\&ED tax credits. In the case of SMEs, this tax advantage could be as high as three times the value of the existing tax benefit from SR\&ED tax credits. This discrepancy was due to the difference in tax treatment of R\&D support from the federal government in relation to these two measures. Indeed, while the federal government applied the rule of reducing firms' eligible expenses for its financial assistance to R\&D by an amount equal to the provincial SR\&ED tax credit benefit, it did not apply such reduction in the case of provincial superdeductions for R\&D.

The superdeductions were effective after years of corporate tax years beginning after June 30, 1999. However, just months after that date and a little less than a year after their announcement by the Government of Québec, the federal government announced in his budget speech in February 2000 amendments to the Income Tax Act to impose the value of a benefit under a provincial superdeduction for R\&D. This meant that the benefit from the superdeductions now gave rise to a decrease in federal support for R\&D as well as in the case of the SR\&ED tax credit. Following the federal government modification, the Government of Québec announced the withdrawal of the superdeductions measure after years of corporate tax years beginning after February 29, 2000.

Despite its relatively short existence, the superdeductions program for $R \& D$ in Québec nevertheless had certain popularity among corporations. Indeed, no less than $12.6 \%$ of companies receiving tax assistance have claimed the superdeductions when they came into force. Moreover, the majority of these companies had claimed tax credits in the year preceding the introduction of superdeduction. The choice of these companies to claim superdeductions instead of tax credits was directly related to the higher tax benefit provided by superdeductions compared to that of refundable tax credits. However, it should be noted that, in principle, the superdeductions targeted profitable companies because they could reduce their tax payable from Québec and enjoy more federal tax assistance. Unprofitable companies had, on their side, benefit to claim the tax credits given the fact that they are fully refundable. 
In this chapter we seek to determine, using data from the program of superdeductions, if firms already receiving significant tax assistance are encouraged to increase their R\&D investments when tax assistance is increased significantly. Specifically, we seek to determine whether companies receiving the superdeductions the surplus tax gain of this new aid provided in addition to the tax credits on to their R\&D spending. From the methodological approach, the impact of the Québec superdeductions program on the R\&D effort of companies who made this choice will be assessed. For that, we compare the average change of R\&D intensity (expressed as the ratio of R\&D spending to sales) of companies that have benefited from the superdeduction program to that of non-recipients using estimates based on the conditional semiparametric difference-in-differences estimator (CDiD).

In the rest of this article we first present the general portrait of the Québec superdeductions program before exposing the main steps of our methodological approach. In the subsequent section, we present the data used and the empirical analysis. We then present the results before concluding.

\subsection{General Portrait of the Program of Superdeductions}

\subsubsection{Eligibility for Assistance}

A company operating a business in Canada and conducting research in Québec, or a company that has research conducted in Québec on its behalf, could receive an additional deduction in computing its income in addition to the usual deduction that applies to the total current expenditure.

\subsubsection{Nature of Assistance}

The following four categories of expenditure entitled each to a superdeduction:

- Wages paid to employees who devote $90 \%$ or more of their time on R\&D activities or R\&D support.

- The expenditure incurred under a research contract or university research conducted by a research center or a public research consortium.

- $\quad$ Expenditures for a pre-competitive research project.

- The dues and fees paid to a research consortium.

These categories of expenditure were in fact those already used by Revenu Québec to qualify for the four types of SR\&ED tax credits. This meant that for a given category of expenditure made, a company could choose between a refundable tax credit or a superdeduction in computing its income. Rates applying to superdeductions were tuned to those of SR\&ED tax credits in order to keep the cost of the financial assistance from the Government of Québec under both fiscal 
measures equivalent. Table 7.2-1 shows the rates of these two tax measures by category of eligible expenditures and the size of the company. For example, an SME that chose superdeductions could apply the rate of $460 \%$ on wages paid to employees working in $R \& D$ and deduct the implied amount from its income. Similarly, that SME would obtain an equivalent tax gain by using the rate of tax credit of $40 \%$ if it claimed SR\&ED tax credits instead.

Table 7.2-1: $\quad$ Superdeductions rates vs SR\&ED tax credit rates, 1999 rates

\begin{tabular}{|c|c|c|c|c|}
\hline & \multicolumn{2}{|r|}{$\mathrm{SME}^{\mathrm{a}}$} & \multicolumn{2}{|c|}{ Large corporation $^{\mathrm{b}}$} \\
\hline & Tax credit & t Superdeduction & Tax credit & uction \\
\hline & \multicolumn{4}{|c|}{$\%$} \\
\hline Credit for salaries and wages $^{c}$ & $40^{d}$ & $460^{\mathrm{e}}$ & 20 & 230 \\
\hline $\begin{array}{l}\text { R\&D work contracted out to: } \\
\text { University entity } \\
\text { Public research centre } \\
\text { Research consortium }\end{array}$ & 40 & 460 & 40 & 460 \\
\hline $\begin{array}{l}\text { R\&D work carried out by two or more } \\
\text { corporations : } \\
\text { Pre-competitive research project } \\
\text { Catalyst project } \\
\text { Environmental technology innovation project }\end{array}$ & 40 & 460 & 40 & 460 \\
\hline R\&D work carried out by a research consortium & 40 & 460 & 40 & 460 \\
\hline
\end{tabular}

Source: Ministère des Finances du Québec, compiled by the author.

Notes:

${ }^{\text {a }}$ Corporations with assets under $\$ 25$ million.

${ }^{b}$ Corporations with assets of $\$ 50$ million or more.

${ }^{c}$ For corporations with assets between $\$ 25$ and $\$ 50$ million the following applies:

- A linear reduction in the rate of the tax credit from $40 \%$ to $20 \%$.

- A linear reduction in the rate of the superdeduction from $460 \%$ to $230 \%$.

${ }^{d}$ Beyond CAN $\$ 2$ million dollars worth of salaries of R\&D, the rate of the tax credit is $20 \%$.

e Beyond CAN $\$ 2$ million dollars worth of salaries of R\&D, the rate of the superdeduction is $230 \%$.

As noted above, the reduction of the cost of $R \& D$ arising from the superdeductions was greater than that from the SR\&ED tax credits due to the differential in the fiscal treatment applied by the federal government to both measures. The Ministère des Finances du Québec estimated to \$27 the cost of a net expense of R\&D salaries of $\$ 100$ for an SME choosing the SR\&ED tax credit for R\&D salaries and only $\$ 9$ if it had chosen instead the superdeductions for R\&D salaries (Table 7.2-2). 
For companies, the coexistence of these two measures had the advantage to ensure that a company making sufficient profits was better off choosing the superdeduction in order to reduce its tax liability to zero. Instead, a company making a negative profit was edge to choose the refundable tax credit to R\&D.

Table 7.2-2: Comparison between the superdeduction and SR\&ED tax credit: net cost for a corporation of an expenditure of R\&D salaries of CAN $\$ 100$ incurred in Québec, 1999

\begin{tabular}{llccc}
\hline & \multicolumn{3}{c}{ SME } & \multicolumn{2}{c}{ Large corporation } \\
\cline { 2 - 5 } & Tax credit & Superdeduction & Tax credit & Superdeduction \\
\cline { 2 - 5 } & & & $(\$)$ & 100 \\
\hline Expenditures & 100 & 100 & 100 & - \\
\hline Québec tax credit & -40 & - & -20 & -21 \\
\hline Superdeduction & - & -41 & - & -20 \\
\hline Federal tax credit & -21 & -35 & -16 & -7 \\
Reduction of Québec's tax credit & -7 & -6 & -8 & -18 \\
\hline Reduction of Federal tax credit & -5 & -9 & -14 & 34 \\
\hline Net cost for the corporation & 27 & 9 & 42 & \\
\hline
\end{tabular}

Source : Ministère des Finances du Québec, Une stratégie fiscale intégrée pour l'économie du savoir, 1999.

\subsubsection{Cost of Superdeductions and Characteristics of Firms receiving the Superdeductions}

Table 7.2-3 shows the value of tax assistance the Government of Québec received by companies under the superdeductions program and other types of tax incentives measures along with the number of beneficiaries by type of assistance. As it can be seen, the year 2000 was the major year for the superdeductions program. Indeed, no fewer than 585 companies have benefited from the superdeductions program (or $12.6 \%$ of companies receiving tax assistance for R\&D from the Government of Québec), which represents a sum of $\$ 77.1$ million (or $14.6 \%$ of the total value of tax assistance for R\&D in 2000). By contrast, in 1999 and in 2001, there were respectively only 2 and 54 recipients companies. This is explained by the fact that most of the period during which the program was in effect (June 30, 1999 to February 28, 2000) coincided with the 2000 tax year for most recipient companies. Taking into account these facts, one can consider that the superdeductions program had certain popularity among corporations. Companies performing R\&D that did not claim the superdeductions are likely those that had little or no knowledge of this program. Other companies that did not participate are those that refrain to do so 
because of difficulties in complying with administrative rules of the program (this which is often the case for small companies) or those that simply have not made enough profits to enjoy the tax advantage of this program.

Table 7.2-3: Cost of superdeductions and of other fiscal incentives to R\&D granted by the Government of Québec and number of recipients enterprises, 1999-2002

\begin{tabular}{|c|c|c|c|c|}
\hline \multirow[b]{2}{*}{ Type of fiscal incentive } & \multicolumn{4}{|c|}{ (k\$ current) } \\
\hline & 1999 & 2000 & 2001 & 2002 \\
\hline - Superdeductions for R\&D & 7 & 77097 & 45267 & 0 \\
\hline - SR\&ED tax credit & 387057 & 423335 & 559609 & 629491 \\
\hline - Tax credit for large corporations & 39174 & 3596 & 0 & 0 \\
\hline $\begin{array}{l}\text { - Tax credit based on the increase in R\&D } \\
\text { expenditures }\end{array}$ & 139 & 24844 & 44794 & 43554 \\
\hline \multirow[t]{3}{*}{ TOTAL } & 426377 & 528872 & 649670 & 673045 \\
\hline & \multicolumn{4}{|c|}{ Number of corporations ${ }^{a}$} \\
\hline & 1999 & 2000 & 2001 & 2002 \\
\hline - Superdeductions for R\&D & 2 & 585 & 54 & 0 \\
\hline - SR\&ED tax credit & 4167 & 4319 & 5167 & 5978 \\
\hline - Tax credit for large corporations & 241 & 34 & 0 & 0 \\
\hline $\begin{array}{l}\text { - Tax credit based on the increase in R\&D } \\
\text { expenditures }\end{array}$ & 13 & 1700 & 3261 & 3885 \\
\hline TOTAL (at least one fiscal incentive) ${ }^{b}$ & 4167 & 4646 & 5199 & 5978 \\
\hline
\end{tabular}

Source: Ministère des Finances du Québec, compilation by the Institut de la Statistique du Québec.

Notes:

a The numbers of recipients companies are slightly underestimated because compilations exclude beneficiaries whose business number in Québec (NEQ) is unknown. These cases account for between $2 \%$ and $5 \%$ of the total tax assistance, by year.

${ }^{b}$ Each company receiving one or more types of tax assistance in a given year is counted only once.

Table 7.2-4 shows the distribution by class of employees of companies carrying out R\&D that have benefited from superdeductions in 2000. As it can be seen, most claims came from small companies. Indeed, at least $72.4 \%$ of companies that have benefited from superdeductions in $\mathbf{2 0 0 0}$ are companies with fewer than 200 employees. However, the total value of the financial assistance received by these companies is just $22.2 \%$. In contrast, the group of companies with over 500 employees that account only $7.6 \%$ of the total number of beneficiaries is the group that received the greatest amount of the total value of aid granted with more than $64.1 \%$ thereof. 
Table 7.2-4: Distribution of the value of superdeduction by size class, 2000

\begin{tabular}{lcc}
\hline Number of employees & Number of recipients & Value of the superdeduction \\
\cline { 2 - 3 } 500 employees and more & $\%$ & $\%$ \\
\hline 200 to 499 employees & 7.6 & 64.1 \\
\hline 50 to 199 employees & 9.7 & 7.9 \\
\hline Less than 50 employee & 23.9 & 12.5 \\
Unknown & 48.5 & 9.7 \\
\hline TOTAL & 10.3 & 5.8 \\
\hline
\end{tabular}

Source : Revenu Québec; Statistics Canada, RDCl survey, compilation by the Institut de la Statistique du Québec and the author.

Examination of the companies receiving superdeduction in 2000 by major business line shows a breakdown a little more balanced between the number of beneficiary companies and the value of aid (Table 7.2-5).

Table 7.2-5: Distribution of the value of superdeduction by sectors, 2000

\begin{tabular}{lcc}
\hline & Number of recipients & Value of the superdeduction \\
\cline { 2 - 3 } Activity sector & $\%$ & $\%$ \\
\hline Manufacturing & $\mathbf{5 6 . 8}$ & $\mathbf{5 8 . 5}$ \\
\hline High technology & 7.6 & 40.9 \\
\hline Medium-high technology & 17.3 & 7.7 \\
\hline Medium-low and low technology & 31.9 & 9.9 \\
\hline Primary and construction & & 1.6 \\
\hline Services & 4.6 & 34.1 \\
\hline Unknown & $\mathbf{2 8 . 3}$ & $\mathbf{5 . 8}$ \\
\hline TOTAL & 10.3 & 100.0 \\
\hline
\end{tabular}

Source: Revenu Québec, Statistics Canada, RDCl survey, compilation by the Institut de la Statistique du Québec and the author. 
Not surprisingly, the manufacturing sector is one that tops the number of beneficiary companies with more than $56.8 \%$ of the total number of beneficiaries and $58.5 \%$ of the total value of aid. This is mainly due to strong over-representation of high technology companies regarding the value of superdeduction. Indeed, although their proportion does not exceed $8 \%$ of the total number of recipient companies, these companies are those that have received the bulk of the total value of aid with more than $40.9 \%$ of it. The service sector and primary industries and construction follow with respectively $34.1 \%$ and $1.6 \%$ of the total value of aid.

\subsection{Methodological Approach}

Our methodological approach is based on the conditional difference estimator of differences CDiD described in Section 6.3 in Chapter 6 based on matching techniques (difference-in-differences matching or DID matching). This estimator is often used in the statistical methods of program evaluation to estimate the difference in the mean of a performance measurement between two distinct moments in time, typically the period before and the period after the start of a program for participants and non-participants, and then comparing the differences between the two groups. In other words, this estimator seeks to subtract from the difference "before/after" of a measure of performance achieved by the program participants, the difference "before/after" of the same measure of performance achieved by non-program participants. The gap is calculated as the DID or more precisely measure the average effect of program participants or "Average Treatment Effect on the Treated (ATT)". This method is often called a "natural experiment" because of the fact that it seeks to construct a comparison group (or control group) with the same properties as the group of program participants from the observed data (as opposed to experimental data).

Formally, as derived in Section 6.3, the average effect of program participants is given by:

$\tau_{D i D}=\frac{1}{N} \sum_{i \in\left(T_{g}=1\right)}\left[Y_{i, t 1}^{1}-Y_{i, t 0}^{1}-\sum_{j \in\left(T_{g}=0\right)} w_{i j}\left(Y_{j, t 1}^{0}-Y_{j, t 0}^{0}\right)\right]$

The double difference of the Equation (7-1) allows the removal of temporal effects common and specific effects that can affect individual participants, thereby skewing the ATT estimator. This is an important element that constitutes the strength and robustness of this estimator compared to other estimators. However, this assumes that these temporal effects common and specific individual must be 
invariant between the two periods, which in some circumstances could be a strong enough case.

Before estimating this equation, an important question concerns the construction of the comparison group. Indeed, special attention must be given to the choice of individuals to include in this group since Equation (7-1) assumes that the control group is similar to the group of participants. However, the literature acknowledges that the group of non-participants may be systematically different from that of program participants because of characteristics inherent in them such as better absorption capacities enabling them to achieve better performance. Such a situation may occur when the selection process for program participants is not random. As an illustration, we can cite the situation where a government agency responsible for a public subsidy program automatically selects the best projects. In these circumstances, the group of participants will be more efficient, even in the absence of the subsidy. The consequence of this situation is that the result of estimation could be biased artificially upward. This is the problem of selection bias.

The construction process of our comparison group is robust to the problem of selection bias, thanks to a correction by the method of estimation by nonparametric matching based on propensity score (propensity score matching or PSM) developed by Rosenbaum and Rubin (1983). This method is based on the intuitive idea of constructing a comparison group by comparing the performance of program participants to that of non-participants "similar" in terms of their observable characteristics or control variables. These characteristics are observable variables that simultaneously affect the decision to participate in a program and performance of the targeted variable, hence the term of "control variables". These variables determine the propensity of an individual or firm to engage in a given program. Thus, the propensity score is a measure of probability of occurrence of an event conditional upon characteristics inherent to the individual or firm interest. In the present case, the propensity score is the probability that a firm claims a tax credit to $R \& D$. This probability depends on several variables that we see in the next section on empirical analysis. To calculate the propensity score, we used the binary logit model which, with the Probit model, is one of the two probabilistic models frequently used for this purpose ${ }^{34}$.

After calculating a propensity score for each participant and each non-member, one can then proceed with the pairing of these two groups to construct the comparison group to use in estimating Equation (7-1). In this chapter we used two methods of matching to verify the sensitivity of our results to different specifications. The first is the method of matching the "nearest neighbor" (Nearest neighbor matching or NNM) which is a method of matching 1-to-1 (or 1-to-1 matching) in which each participant is only matched to one non-participant at a

\footnotetext{
${ }^{34}$ Details on probabilistic models may be found in Maddala (1983).
} 
time. To do this, the non-participant with the closest propensity score of the participant is selected as "match". The second method used is the matching procedure based on estimating a local linear regression (LLR) developed by Heckman et al. (Heckman et al. (1997), Heckman, Ichimura and Todd (1998b)). This method is more robust than the first and it is a method of matching 1-to-n (or 1-to$\mathrm{n}$ matching) in which each participant can be matched to several non-participants. In this case, a weight is assigned to each non-participant depending on the distance between the propensity score and the participant in the program. It should be noted here that both methods are extreme, between them, there are many other methods, including methods called "caliper matching" and "stratification matching (for a detailed review of matching techniques, see Blundell and Costa Dias (2000) or Sascha and Andrea (2002)). Following the application of either of the methods just described, we obtain a new sample composed of group participants that could be "matched" and the corresponding comparison group. We can then proceed to the last step of estimating Equation (7-1) using the new sample.

The estimation of the Equation (7-1) through the sample obtained by the NNM method is easy to implement in that it does not require the estimation of a regression. In this case the weighting function in Equation (7-1) is given by:

$w_{i j}=\frac{1}{N_{i}}$

The case of the matching procedure based on estimating a local linear regression requires a more complex algorithm as shown in Section 6.3. In this approach, the 1-to-n matching process and the estimation of Equation (7-1) are performed in a single operation so that the differences before/after of the average performance of participants are compared to the differences before/after of the weighted averages of the performance of non-participants. As a reminder, the weighting function in this case is given by:

$$
w_{i j}=\frac{G_{i j} \sum_{k \in(T g=0)} G_{i k}\left(p_{k}-p_{i}\right)^{2}-\left[G_{i j}\left(p_{j}-p_{i}\right)\right]\left[\sum_{k \in(T g=0)} G_{i k}\left(p_{k}-p_{i}\right)\right]}{\sum_{j \in(T g=0)} G_{i j} \sum_{k \in(T g=0)} G_{i j}\left(p_{k}-p_{i}\right)^{2}-\left(\sum_{k \in(T g=0)} G_{i k}\left(p_{k}-p_{i}\right)\right)^{2}}
$$

Intuitively, this term that enters Equation (7-1) as the weighting function for non-participants, attribute to each non-participant a weight based on the distance between the propensity score of the participant and that of the non-participant. This weight will increase as the distance decreases. 
The next section discusses the main features of the data used in this chapter and their application to the methods just described.

\subsection{Data Characteristics}

The data come from merging two data files: the micro-data from the Québec portion of the RDCl Survey and the administrative data of companies receiving tax incentives for R\&D provided by Revenu Québec. The idea of linking these two files found his interest in the nature of the data they contain. The RDCl survey is an annual survey conducted by Statistics Canada to track the evolution of scientific research and technology in Canada. As such, this file contains data on expenditures and personnel devoted annually to $R \& D$ by Canadian industry. A detailed description of the survey RDCl can be found in Statistics Canada (2002). The file of administrative data mainly includes data on tax credits and other types of tax incentives for $R \& D$ received by companies performing $R \& D$ or that have $R \& D$ performed on their behalf in Québec. The merging of both files was performed at the company level since the latter is the reporting unit in both cases. This process is explained in Section 4.3.

Table 7.4-1 shows the number of firms matched in the period from 1998 to 2002 following the linking process mentioned above. This initial sample data included a total of 9665 companies from all sectors of NAICS. We have completed the preparation of the final sample taking into account that this chapter focuses on three years; the year 1999 which is the base or pre-program year ${ }^{35}$ and the years 2000 and 2001 that we call post-treatment years. We excluded companies whose size is located at the ends of the sample, which is the case of companies with fewer than 5 employees and those with more than 1500 employees (482 companies). Companies with values too far from the mean of the sample in the other control variables we used were also excluded (110 companies). Furthermore, we have removed all the companies that had not received tax credit for R\&D in 1999 (about 7083 companies) so as to keep in our final sample only companies that were in a situation similar to the base year 1999 before the introduction of the superdeductions program that is to say, only companies benefiting from tax credits.

\footnotetext{
${ }^{35}$ Although as it appears in Table 7.4-1, some firms have received support from the superdeduction program in 1999, in this study we consider the year 1999 as the base year given the negligible number of companies that received tax assistance this year. Moreover, they have been excluded from this analysis.
} 
Table 7.4-1: Number of superdeductions and of other fiscal incentive recipients to R\&D in the study sample, 1998-2002.

\begin{tabular}{|c|c|c|c|c|c|}
\hline \multirow[t]{2}{*}{ Type of fiscal incentive } & \multicolumn{5}{|c|}{ Number of recipients } \\
\hline & 1998 & 1999 & 2000 & 2001 & 2002 \\
\hline - Superdeductions for R\&D & 0 & 2 & 529 & 48 & 0 \\
\hline - SR\&ED tax credit & 2997 & 3475 & 3801 & 4578 & 5093 \\
\hline - Tax credit for large corporations & 259 & 182 & 26 & 0 & 0 \\
\hline - Tax credit based on the increase in R\&D expenditures & 0 & 10 & 1589 & 2974 & 3404 \\
\hline TOTAL (at least one fiscal incentive) ${ }^{a}$ & 3245 & 3645 & 4141 & 4608 & 5093 \\
\hline
\end{tabular}

${ }^{a}$ Each company receiving one or more types of tax assistance in a given year is counted only once.

Source: Ministère du Revenu du Québec; Statistics Canada, RDCl survey, compilation by the Institut de la Statistique du Québec and the author.

Following the cleaning process data, we obtained a final sample consisting of 1990 companies in the two-year study. In 1999, all companies in the sample received at least one component of the refundable tax credit to SR\&ED. In 2000, 1813 to 1990 companies have continued to receive the tax credit while SR\&ED 177 other companies have substituted the SR\&ED tax credit by superdeduction. This therefore gives us a sample of 177 beneficiary companies (treated companies) and 1813 non-beneficiary companies (untreated companies) for empirical analysis.

\subsection{Assessing the Impact of the Program of Superdeductions}

The evaluation was conducted in two stages. The first stage of the analysis was to calculate the propensity score of each company in the sample. To do this, we estimated a binary probit model in which we included the control variables in the probability that a company seeks tax assistance for R\&D as well as those that may affect $R \& D$ intensity. For that, we included in the regression some of the variables used in the estimation of probit models in the preceding chapter (Section 6.5.1). These include, the lagged log of the number of employees, the intensity of capital constructed as the lag of the logarithm of firm's real assets divided by the number of employees, the share of technical personnel working on R\&D activities constructed as the lagged value of the ratio of firm's R\&D personnel to number of employees, an indicator variable for the country of ownership of the firm and industry dummies. The definition and construction of these variables are given in the preceding chapter (Table 6.A-1). The other two variables included in the probit 
estimation of the preceding chapter that could improve the fit of the present model are the market share and the export share. These variables could not be included in the model since they were not available from the two merged data files from which the dataset used in this chapter was constructed. However, in order to improve the fit of the model, we added the age of the firm that was available for all the firms in the study sample. It is often argued that younger firms are more likely to claim tax assistance because they are facing financial constraints (Czarnitzki and Licht (2006)). If this assumption is verified, we would expect a decrease in the probability of claiming for tax assistance as the firm is getting older.

Table 7.5-1: Probit estimates for the propensity score estimation

\begin{tabular}{lcc}
\hline Parameters/statistics & Probit 1 & Probit 2 \\
\hline$I_{t-1}$ & $0.292^{* * *}$ & $0.297^{* * *}$ \\
& $(0.058)$ & $(0.058)$ \\
$(\mathrm{k} / \mathrm{I})_{t-1}$ & $0.0623^{* * *}$ & $0.0609 * * *$ \\
& $(0.024)$ & $(0.024)$ \\
$\left(\mathrm{L}_{\mathrm{r}} / \mathrm{L}\right)_{\mathrm{t}-1}$ & $0.0211^{*}$ & $0.0196^{*}$ \\
& $(0.011)$ & $(0.011)$ \\
OWN & -0.0713 & $-0.225^{* *}$ \\
& $(0.095)$ & $(0.114)$ \\
$1 /$ age ${ }_{t-1}$ & & $-1.716^{* *}$ \\
Industry dummies & & $(0.737)$ \\
indus1-indus20 & included & Included \\
& & $-3.293^{* * *}$ \\
Intercept & $-3.522 * * *$ & $(0.378)$ \\
Pseudo R2 & $(0.367)$ & 0.141 \\
\hline Log likelihood & 0.136 & -513.06 \\
Chi2 & -515.97 & 168.24 \\
P-value & 162.42 & 0.000 \\
\hline Number of observations & 0.000 & 1990 \\
\hline
\end{tabular}

Notes: $\quad * * *$ indicates significance at $1 \%, * *$ at $5 \%, *$ at $10 \%$. Standard errors in parentheses unless otherwise indicated. Lower case symbol denote the logarithm of the corresponding variable. I:log of number of employees, $k / I$ : capital intensity, $L_{r} / L$ : share of R\&D personnel, OWN: origin country dummy ( Canada=1, Foreign=0), indus1-indus20: industry dummies.

Table 7.5-1 shows the estimation results of the binary probit model in which, we recall, the dependant variable is a dummy variable that takes the value of 1 if a firm received both $R \& D$ tax credits and support from the superdeduction program, and 0 if it received only R\&D tax. Probit Model 2 that includes the complete set of variables mentioned above is compared to Probit Model 1 which does not include the variable capturing the age of the firm. As one can see, in Probit Model 1 all variables are significant except in the case of the variable for the country of 
ownership of the firm. The inclusion of the the age of the firm in Probit Model 2 improves the estimation result and, therefore, all the predictor variables are significant statistically at the $10 \%$ level. Hence, Probit Model 2 is our preferred model. According to the signs of estimated coefficients in the latter, it comes that the probability for a company to receive tax assistance for R\&D increases with the number of employees, the capital intensity and the share of R\&D personnel, and decreases with the age of the company and the degree of foreign ownership. These results confirm those found in Section 6.5.1. Finally, the test for the global null hypothesis indicates that the probit regression model is statistically significant (LR Chi2 $=168.24$, P-value $=0.000$ ), hence giving greater confidence in the validity of the model. This model is then used to compute propensity scores for the 1990 observations in the sample (177 program participants and 1813 non-participants).

The second stage of the analysis is that of matching using the NNM and the LLR matching. Calculating the average effect of the program on the treated companies (or ATT) was performed on the sample of 1949 companies mentioned above. This stage was performed as described in Section 6.5.1. We performed the matching for the two years following the implementation of the superdeduction program (i.e. 2000 and 2001) to ensure that delays in firms' adjustment are taken into account. Following the matching process 176 companies among the 177 beneficiary companies were matched for both the NNM method and the LLR method. As in Section 6.5.1, we imposed that each treated company from a particular sector of activity be matched only to an untreated company belonging to this sector.

The results of the matching are reported in Table 7.5-2. For the first year after the introduction of the program, the estimate of the method of Difference-indifferences based on NNM shows a growth of R\&D intensity of the group of beneficiaries companies of $2.90 \%$ in average while in the case of the group of nonbeneficiaries the growth is $0.69 \%$. The difference between these two values, or 2.20 percentage points, is the CdiD or the average effect of treatment (ATT) on the group of beneficiaries companies. However, the $95 \%$ confidence interval of the t-test on the ATT estimate is very wide and includes the null value, ranging from -39.77 to 38.22. Hence, this interval implies no statistically significant change of the outcome variable. This result is further confirmed by the result of the matching with the more efficient method of the difference-in-differences based on the LLR. In this case, results a more modest treatment effect 0.48 which is also, as in the NNM case, statistically non-significant. For the second year, higher CDiD point estimates are reported than in the first year, i.e. 2.51 for the NNM method and 0.85 with the LLR method. As may be deducted from the values of the bias corrected $95 \%$ confidence interval, the increase in the growth of R\&D intensity is not enough to induce a statistically significant change. 
Table 7.5-2: $\quad$ Estimated average treatment effects (ATT) on R\&D intensity change

\begin{tabular}{|c|c|c|c|c|c|}
\hline \multicolumn{6}{|c|}{ Outcome variable: $R \& D$ intensity } \\
\hline & \multirow{2}{*}{$\begin{array}{c}\text { Pre-program } \\
\text { year }\end{array}$} & \multicolumn{2}{|c|}{ First year } & \multicolumn{2}{|c|}{ Second year } \\
\hline & & NNM & LLR & NNM & LLR \\
\hline $\begin{array}{l}\text { Tax Credits }+ \\
\text { superdeductions } \\
(n=176)\end{array}$ & 2.87 & 2.90 & 2.90 & 3.18 & 3.18 \\
\hline $\begin{array}{l}\text { Tax credits only } \\
(n=1,780)\end{array}$ & 0.57 & 0.69 & 2.36 & 0.67 & 2.33 \\
\hline $\begin{array}{l}\text { Unadjusted mean } \\
\text { Difference }\end{array}$ & 2.30 & & & & \\
\hline CDiD point estimate & & 2.20 & 0.53 & 2.51 & 0.85 \\
\hline 95\% Conf. Interval & & $(-39.77,8.22)$ & $(-4.39,5.91)$ & $(-39.47,38.34)$ & $(-8.65,7.46)$ \\
\hline \multicolumn{6}{|l|}{ Sensitivity analyses } \\
\hline \multicolumn{6}{|l|}{ CDiD point estimate } \\
\hline $\begin{array}{l}\text { Changing bandwidth } \\
\text { default } .8 \text { to a small } \\
\text { bandwidth } .04\end{array}$ & & 2.20 & 0.48 & 2.51 & 0.80 \\
\hline 95\% Conf. Interval & & $(-17.45,39.61)$ & $(-6.41,8.75)$ & $(-17.17,39.78)$ & $(-6.21,9.27)$ \\
\hline $\begin{array}{l}\text { Trimming (5\%) : } 8 \\
\text { obs. excluded }\end{array}$ & & 1.32 & 0.29 & 1.63 & 0.62 \\
\hline 95\% Conf. Interval & & $(-25.49,89.07)$ & $(-6.15,8.40)$ & $(-25.23,104.83)$ & $(-5.80,8.75)$ \\
\hline
\end{tabular}

Note: $\quad$ Standards errors of all CDiD point estimates are bootstrapped with 500 replications.

To check for the robustness of the matching results, we conducted sensitivity analyses to test how the latter varies when using different specifications in the matching process. We did this, on the one hand, by changing the values of the bandwidth parameter and, on the other hand, through trimming the sample, in order to impose a common support, by dropping a certain percent of treated cases at which the pscore density of the nontreated cases is the lowest (see PSMATCH2 propensity score matching statistical module by Leuven and Sianesi (2003) for more details on trimming and bandwidth parameters in the estimation of LLR tricube kernel). The results show that, for example, by changing the default value of 0.8 on the bandwidth parameter to a small bandwidth 0.04, the CDiD point estimate changes slightly only in the case of LLR, but in all cases the CDiD point estimates are not statistically significant at a confidence level of $95 \%$. Finally, trimming the sample by $5 \%$ drops 8 treated observations in all cases and the CDiD point estimates remain statistically non-significant.

In addition to the sensitivity tests, we performed the usuals before/after group means significance tests. These tests consist of estimating statistically the difference 
between the group of participants and that of non-participants, thus allowing evaluating the success of the matching process. Matching is successful if no significant differences between the means of the two groups remain after its application. Two tests were performed, the t-test of group mean comparison and the standardized difference statistic, described in Section 6.5.1. Table 7.5-3 and Table 7.5-4 show the balancing tests before and after performing propensity score matching. The results for the t-statistic show that before the matching, for all covariates, except for the indicator variable for firm ownership, there is a strong statistical difference between the two groups of means (Table 7.5-3), including the propensity score. However, for the response variable, R\&D intensity, the test result indicates no statistical difference between both groups. This is consistent with the fact that the matching did not yield statistically significant CDiD point estimates.

Table 7.5-3: Balancing tests before propensity score matching, all firms

\begin{tabular}{|c|c|c|c|c|}
\hline \multirow{3}{*}{ Variable } & \multicolumn{2}{|c|}{ Group mean } & \multicolumn{2}{|c|}{ Group means differences } \\
\hline & $\begin{array}{c}\mathrm{Tg}=1 \\
\text { Tax Credits + } \\
\text { superdeductions }\end{array}$ & $\begin{array}{c}\mathrm{Tg}=0 \\
\text { Tax credits only }\end{array}$ & t-statistic & $\begin{array}{r}\text { Standardized } \\
\text { difference (\%) }\end{array}$ \\
\hline & N1=177 & N0=1 813 & & \\
\hline \multirow{2}{*}{ AGE } & 16.373 & 13.466 & $3.779 * * *$ & 28 \\
\hline & $(0.820)$ & $(0.227)$ & 0.000 & \\
\hline \multirow{2}{*}{ L } & 159.412 & 58.722 & $10.194 * * *$ & 59 \\
\hline & (15.922) & $(2.668)$ & 0.000 & \\
\hline \multirow{2}{*}{$\mathrm{K} / \mathrm{L}$} & $2.2 e+05$ & $1.5 e+05$ & $1.903^{*}$ & 14 \\
\hline & $(4.2 e+04)$ & $(1.0 \mathrm{e}+04)$ & 0.057 & \\
\hline \multirow{2}{*}{$\mathrm{L}_{\mathrm{r}} / \mathrm{L}$} & 10.382 & 5.055 & $11.069 * * *$ & 93 \\
\hline & $(0.391)$ & $(0.145)$ & 0.000 & \\
\hline \multirow{2}{*}{ OWN } & 0.322 & 0.271 & 1.456 & 11 \\
\hline & $(0.035)$ & $(0.010)$ & 0.146 & \\
\hline \multirow{2}{*}{ PSCORE } & 1.982 & 2.616 & $-10.727^{* * *}$ & -82 \\
\hline & $(0.060)$ & (0.018) & 0.000 & \\
\hline \multirow{2}{*}{$R / Q$} & 2.962 & 0.570 & 0.138 & 1 \\
\hline & (1.143) & (5.338) & 0.890 & \\
\hline
\end{tabular}

Note: $\quad * * *$ indicates significance at $1 \%, * *$ at $5 \%, *$ at $10 \%$. Standard errors in parentheses unless otherwise indicated. The descriptive statistics are sample means for the years 1997-2004. The base year is 2002. Lower case symbol denote the logarithm of the corresponding variable. AGE: age of firm, L: number of employees, $K / L$ : log capital intensity, $L_{r} / L$ : share of $R \& D$ personnel, OWN: origin country dummy (Canada=1, Foreign=0), PSCORE: propensity score, $R / Q: R \& D$ intensity.

After the matching, the differences in the means of the two groups in all covariates and in the propensity score are no longer statistically significant 
(Table 7.5-4). Results from the standardized difference statistic confirm these findings as after the matching all covariates lie below the $10 \%$ threshold (except for the number of employees where the statistic reported is $12 \%$, which is close to the threshold). We may therefore conclude to the the robustness of the matching methods and the consistence of the CDiD point estimates.

Table 7.5-4: Balancing tests after propensity score matching, all firms

\begin{tabular}{|c|c|c|c|c|}
\hline \multirow{3}{*}{ Variable } & \multicolumn{2}{|c|}{ Group mean } & \multicolumn{2}{|c|}{ Group means differences } \\
\hline & \multirow{2}{*}{$\begin{array}{c}\mathrm{Tg}=1 \\
\text { Tax Credits + } \\
\text { superdeductions } \\
\mathrm{N} 1=177\end{array}$} & \multirow{2}{*}{$\begin{array}{c}\mathrm{Tg}=0 \\
\begin{array}{c}\text { Tax credits } \\
\text { only }\end{array} \\
\mathrm{N0}=1813\end{array}$} & \multirow[t]{2}{*}{ t-statistic } & \multirow[t]{2}{*}{$\begin{array}{c}\text { Standardized difference } \\
(\%)\end{array}$} \\
\hline & & & & \\
\hline \multirow{2}{*}{ AGE } & 16.536 & 18.042 & -1.239 & -15 \\
\hline & $(0.851)$ & $(0.868)$ & 0.216 & \\
\hline \multirow{2}{*}{ L } & 156.101 & 134.893 & 1.021 & 12 \\
\hline & $(14.732)$ & $(14.638)$ & 0.308 & \\
\hline \multirow{2}{*}{$\mathrm{K} / \mathrm{L}$} & $2.3 e+05$ & $2.9 e+05$ & -0.779 & -12 \\
\hline & $(4.4 e+04)$ & $(6.7 e+04)$ & 0.437 & \\
\hline \multirow{2}{*}{$\mathrm{L}_{\mathrm{r}} / \mathrm{L}$} & 0.849 & 0.986 & -1.068 & -2 \\
\hline & $(0.090)$ & $(0.092)$ & 0.286 & \\
\hline \multirow{2}{*}{ OWN } & 0.875 & 0.917 & -1.249 & -9 \\
\hline & $(0.026)$ & $(0.021)$ & 0.212 & \\
\hline \multirow{2}{*}{ PSCORE } & 1.978 & 1.979 & -0.009 & -0 \\
\hline & $(0.057)$ & $(0.057)$ & 0.993 & \\
\hline \multirow{2}{*}{$\mathrm{R} / \mathrm{Q}$} & 2.989 & 0.412 & 0.555 & 2 \\
\hline & (1.149) & $(4.500)$ & 0.579 & \\
\hline
\end{tabular}

Note: $\quad * * *$ indicates significance at $1 \%, * *$ at $5 \%, *$ at $10 \%$. Standard errors in parentheses unless otherwise indicated. The descriptive statistics are sample means for the years 1997-2004. The base year is 2002. Lower case symbol denote the logarithm of the corresponding variable. AGE: age of firm, L: number of employees, $K / L$ : Capital intensity, $L_{r} / L$ : share of $R \& D$ personnel, OWN: Origin country dummy (Canada=1, Foreign=0), PSCORE: Propensity score, R/Q: R\&D intensity.

The results presented above are in line and adds to the evidence on the previous results found in Baghana (2008) where the matching was performed only for the first year after the introduction of the program. In this study, no statistically significant effect could be found from the matching.

In conclusion, even though the R\&D intensity effort of firms that received the superdeductions seems slightly higher than that of other firms, the statistical evidence does not confirm that firms already receiving some tax assistance continue to will increase their R\&D investments in the event of tax assistance increases. Thus, an important policy implication of the results is that there is a certain threshold 
beyond which government funding become less effective. Guellec and Van Pottelsberghe (2000) arrived at the same conclusion in a study on stimulated business-funded R\&D in 17 OECD countries over the period 1981-1996. Accordingly, the phenomenon of crowding-out cannot be totally excluded in the case of Québec superdeductions and this is mainly because the tax assistance is level-based. Yet, as we showed in Chapter 5, the incremental-based fiscal measures suffer less from this problem.

\subsection{Conclusions}

The main question addressed in this chapter is whether firms already benefiting from tax assistance increase their R\&D spending following a significant increase of the tax assistance. We answer this question by studying the case of Québec firms to whom the government offered through the program of superdeductions a tax benefit of up to three times the value of the existing tax benefit. More specifically, the question asked in this chapter is that of knowing whether the reduction of the marginal cost of R\&D led by the superdeductions program in Québec had prompted the companies already benefiting from the SR\&ED tax credit advantage to increase their R\&D effort. The results obtained show that there is no evidence that firms already receiving some tax assistance will increase their R\&D investments in the event tax assistance is significantly increased. Accordingly, the phenomenon of crowding-out cannot be totally excluded in the case of Québec superdeductions, at least in the short run, and this is mainly because the tax assistance is level-based. The incremental-based fiscal measures suffer less from this problem. However, it may be argued that this negative result is presumably due to adjustment delays related to the fact that $R \& D$ investments in companies are typically undertaken in multi-year programs. Unfortunately, the relatively short duration of the superdeductions program does not permit to test the effects of this program in the long term.

Several other issues could be analyzed to complete this chapter. Among these issues there is for example the estimation of the impact of the superdeductions program on recipient companies that received no tax support before the introduction of the superdeductions program. However, data files available to us contained no relevant information to conduct such an analysis. Also, we could have done the analysis by size class to see in what group size the problem is most severe, but relatively small subsamples have forced us to abandon this option. This option would therefore be an interesting avenue for future research. 


\section{CHAPTER 8}

\section{Impact of Government \\ Funding on R\&D, Innovation, and Productivity: Results from the Innovation Survey Microdata in Québec}

Rufin Baghana

Geneviève Renaud*

* Institut de la Statistique du Québec

Submitted for publication 


\subsection{Introduction}

Technological innovation is believed to be one of the key elements explaining the collective wealth and job creation. This belief has even strengthened in recent years notably because of the necessity for all the countries to have a competitive economy in front of the imperatives of the globalization as well as the knowledge economy. Thus, several countries or even some regional groupings have adopted national or regional policies based primarily on direct and indirect public subsidies to stimulate innovation and hence productivity. To assess the validity and effectiveness of these policies, both microeconomic and macroeconomic studies attempted to establish the direct link between public support and innovation on the one hand, and between public support and productivity, on the other hand. At the microeconomic level in which the present study falls, several studies have found a positive link between public assistance granted to businesses and innovation. These include for example, Czarnitzki and Hussinger (2004), Czarnitzki et al. (2005), Czarnitzki and Licht (2006), Bérubé and Mohnen (2009), Garcia and Mohnen (2010).

However, despite these encouraging results, the question of knowing whether firms benefiting from government support invest on average more on innovation than those that don't receive support still remains. There are cases where the socalled crowding-out phenomenon is present as shown in some studies. Moreover, this phenomenon has given rise to a formidable literature in the late 90 s and early 2000s (see Klette et al. (2000) or David et al. (2000) for some review). Thus, the evidence on the issue is mixed and we believe that this will remain unchanged since the review of the literature shows a heterogeneity such that, results vary from one country to another, from one jurisdiction to another and even from one program to another within the same jurisdiction or country. For example, we can cite Wallsten (2000) who shows that young US firms in the technology sector have reduced their R\&D spending after being funded under the Small Business Innovation Research program (SBIR) or, Lach (2002) who, using data on Israeli manufacturing firms, concludes that $R \& D$ subsidies stimulated long-run company-financed R\&D expenditures, but this positive effect however, is inferior to what was expected. Thus, one can assume that the existence or not of crowding-out or its severity (partial crowding-out vs. total crowding-out) appears to be directly related to the types of programs and policies, their objectives or to their rules. Investigation in each case is therefore necessary to determine whether a particular program or policy provides the anticipated results or not. More specifically, whether, on the one hand, subsidized firms invest on average more on innovation than nonsubsidized firms and, on the other hand, if these firms perform better in terms of productivity. 
Another concern addressed by this study is the question of what causes the lag experienced by Québec in its labour productivity compared to other Canadian provinces and major industrialized countries. Several studies have addressed this issue which remains valid for several decades (e.g.: Green (1971), Bélanger (1980), Davenport (1981), Migué (1998), Paquet (1999), Boyer (2001), Fortin (2001), Tessier and Lagacé (2003)). Among these studies, some have pointed to shortcomings in the productivity of Québec businesses as one of the elements explaining this mystery. In a recent study, the HEC Montréal Centre for Productivity and Prosperity (CPP (2010)) shows that, among these causes, is the fact that Québec industry is turned towards low-productivity sectors and that the sector of goods production contributed poorly to labour productivity in the last years. This study examines this puzzle by comparing the performance of Québec's manufacturing firms to those of major industrialized countries in order to check if the first are underperforming.

Finally, another objective of this study is to fill the gap in the literature regarding the evaluation of the effectiveness of government support for innovation in Québec which, it must be remembered, is among the most attractive in the world (see for example Warda (1999)). Indeed, if at the Canadian level a few attempts have been made to answer these questions ${ }^{36}$, it has not been the case for Québec: To our knowledge, this is the first study using Québec data to examine these issues empirically. Baghana (2010) addresses these issues with Québec data, but in this case, the analysis is limited to the evaluation of the effects of direct public funding, namely R\&D subsidies, on firms R\&D intensity and productivity. In this study however, the emphasis is rather on the combined effects of direct and indirect subsidies on innovation output (innovation sales) and on productivity.

One of the main advantages of this study is that, unlike most studies, it uses a unique and comprehensive dataset that includes administrative data on the amounts of government support actually received by firms. Indeed, a common criticism is the fact that most studies use data from innovation surveys which mostly do not include the actual amount of government support received by firms. The consequence of the absence of this variable is that these studies are often constrained to use only a dummy indicating whether or not a firm received public support. This severely limits the scope of these studies.

To carry out this study, we use, as mentioned above, a broad and rich dataset from multiple sources consisting of an initial sample composed of an unbalanced panel of 3161 manufacturing firms in Québec over the period 2002-2004. As regards to the methodological approach, our starting point is the OECD core model (OECD (2009)) which is a multi-country study based on data from 17 OECD

\footnotetext{
${ }^{36}$ Studies exploring the relationship between public assistance paid to businesses and innovation using Canadian data include Czarnitzki et al. (2005), Bérubé and Mohnen (2009).
} 
countries $^{37}$ and one major developing economy, Brazil. The OECD model uses harmonized data from the $\mathrm{CIS}^{38}$ in the case of European Union countries and from surveys on innovation carried out by national statistical offices in the case of the other participating countries. The OECD core model is an extension of the model described by Crépon et al. (1998), also known as the CDM model. In this study, we further extend the OECD core model to explicitly include a function of government support in a way to effectively assess the effect of public incentives on innovation as well as on productivity. At this point, it should be noted that this study examines the total effect rather than the effect of specific type of government assistance because Québec firms can benefit simultaneously from multiple type of public support for innovation, including $R \& D$ tax credits and R\&D grants.

The remainder of this study consists of six sections. In Section 8.2, as this study is based on a CDM-type model, we provide a brief summary of the main results found in studies using this model in recent years. Section 8.3 presents the characteristics of innovative firms in Québec. Section 8.4 devoted to the methodological approach first presents the OECD core CDM model which is our starting model and then introduces the extended CDM model for Québec. Section 8.5 describes the data sources, the construction of the database and the variables used. Section 8.6 presents in two sub-sections the results found: in the first sub-section, the results of the extended model for Québec are presented. In the second sub-section, the results of the OECD core model estimated on Québec data are presented and compared to the results found for the 18 countries included in the OECD study. Finally, Section 8.7 presents a summary of the work before concluding.

\subsection{Literature Review}

The CDM model is a system of three recursive equations that links research efforts to innovation output and productivity. The first stage models the firm's decision to engage in research activities and the amount of efforts devoted to it. The second stage estimates the knowledge production function, i.e. the effect of research and other innovation inputs on innovation output (patents or innovation sales). The last stage estimates the effect of innovation output on productivity. The CDM model has been widely used since the 1998 seminal paper by Crépon, Duguet and Mairesse.

\footnotetext{
${ }^{37}$ Australia, Austria, Belgium, Canada, Denmark, Finland, France, Germany, Italy, Korea, Luxembourg, Netherlands, New Zealand, Norway, Sweden, Switzerland, United Kingdom.

${ }^{38} \mathrm{CIS}$ is the acronym for Community Innovation Surveys which are a series of surveys executed by national statistical offices throughout the European Union and in Norway and Iceland. The harmonized surveys are designed to give information on the innovativity of different sectors and regions.
} 
However, the results obtained in these studies are hardly comparable because several extensions have been introduced and this, in different directions.

Hence, while the original CDM model focused on product innovation as output, number of paper introduced other types of innovation output, for example, process and non-technological innovation. Other extensions rather concentrated on innovation input by replacing or adding to R\&D other types of input. In the original CDM model, R\&D capital was specified as innovation input in the knowledge production function. The number of patents and the share of innovation sales were used as "proxies" for innovation output; both proxies turned out to have a positive and significant effect on productivity. Following studies used R\&D intensity instead of R\&D capital in the knowledge production function. These include Griffith, Huergo, Mairesse and Peters (2006) and Hall, Lotti and Mairesse (2009). Additionally, these studies added investment intensity as innovation input. The former also added appropriability conditions, information sources and a proxy for demand pull. Both papers found similar results using two separate indicators to proxy innovation output; that is the probability of introducing a product innovation and the probability of introducing a process innovation. Results indicate positive and significant effect of the product output on productivity ${ }^{39}$ and no effect for the process output ${ }^{40}$.

Parisi, Schiantarelli and Sembenelli (2006) found opposite results using dummy variables instead of probabilities for introducing product and process innovations to proxy innovation output: the product output had no significant effect on productivity while the process output had a positive and significant effect. Polder, Leeuwen, Mohnen and Raymond (2009) also used dummy variables to proxy innovation output. Their results indicate that the introduction of a product or a process innovation has to involve an organizational innovation to have a significant effect on productivity. Their extension of the CDM model includes ICT inputs in the knowledge production function ICT investments being access to broadband and dummy variables indicating whether a firm has done e-sales and e-purchases. van Leeuwen and Farooqui (2008) also included ICT inputs in the knowledge production function, i.e. an indicator for broadband connectivity and the amount of the firm's e-sales. Using Dutch data, they found an elasticity of productivity with respect to innovation output (proxied by innovation sales per employee) of 0.14.

The OECD (2009) model, which was built primarily with the objective to allow comparison between countries, was estimated on a set of variables slightly reduced to ensure that it is usable on data from a large number of countries. The model main variables include $R \& D$ expenditures, process innovation and cooperation in

\footnotetext{
${ }^{39}$ Griffith et al. (2006) found a positive and significant effect for France, Spain and the UK and no significant effect for Germany.

${ }^{40}$ Griffith et al. (2006) found a positive and significant effect for France and no significant effect for Germany, Spain and the UK.
} 
innovation as innovation inputs and labour productivity, proxied by the ratio of sales per employee. The elasticities found for the 18 countries range from 0.23 for Luxembourg to 0.69 for Korea. The elasticity found for Canada is 0.43 . Likewise, using Canadian data on a similar model but extended to include a richer set of variables including human capital and value added, Therrien and Hanel (2009) found an elasticity of 0.22 , which is about half of the value estimated in the OECD core model. Following the OECD project also, Johansson and Lööf (2009) estimated elasticities between 0.36 and 0.52 with Swedish data and Berger (2009) did the same with Thai data and reported elasticities between 0.54 and 1.22.

Finally, other papers estimating the elasticity of productivity with respect to innovation sales per employee include: Lööf, Heshmati, Asplund and Naas (2001), Lööf and Heshmati (2006) and OECD (2009). For Finish firms, Lööf et al. (2001) found no significant effect of innovation output on productivity, but they found positive and significant elasticities ranging from 0.10 to 0.26 for Norwegian and Swedish firms. The corresponding elasticities produced by Lööf and Heshmati (2006) for Swedish firms in the manufacturing and services sectors were positive and significant in both sectors with a greater magnitude in the manufacturing sector.

Although constructed from the OECD model and including most of the variables as those used by Therrien and Hanel (2009), the model used in this study differs from previous studies. Unlike these, it models explicitly a function of government support into the standard CDM model in order to estimate the effect of government incentives on R\&D investment, innovation and productivity. Consequently, the results apply to the restricted sample of beneficiaries of government incentives. The review of the literature concerning studies that used such a model is scarce. Among the few studies available, we found for example the study by Halpern and Muraközy (2010) that uses Hungarian CIS data on a CDM model in which is included, among explanatory variables, a binary for government support in all the equations. The results show a strong positive relationship between government subsidies and innovation in general and the elasticity of productivity with respect to innovation sales per employee of 0.33 .

\subsection{Patterns of Innovation in Québec}

\subsubsection{Characteristics of Innovative Firms}

The characteristics of firms described in this section are based on the results from the Canadian survey of innovation 2005, which we refer as IS2005, that covers the years 2002 to 2004 (see detailed description in Section 4.2.3). The IS2005 comprises two types of innovation, that is, product and process innovations. The survey questionnaire defines a product innovation as the market introduction of a new or 
significantly improved good or service within the last three years. The innovation, new or improved, must be new to the firm and should exclude the simple resale of new goods purchased from other plants and changes of a solely aesthetic nature. A process innovation is defined as the implementation of a new or significantly improved production process or distribution method for goods or services, or support activity for processes, within the last three years. It is also required that the process innovation be new to the firm. To distinguish between different types of product innovators, the survey questionnaire asks if the new or significantly improved products introduced by the firm were also new to the market. In addition, it asks if any product and any process innovation introduced by the firm was a first in Québec, in Canada, in North America or a world-first.

Table 8.3-1: Distribution of innovating firms in Québec's manufacturing sector, 2002-2004

\begin{tabular}{|c|c|c|c|c|}
\hline & & Number of firms & $\%$ of all firms & $\%$ of innovators \\
\hline \multicolumn{2}{|c|}{ All firms } & 4310 & 100.0 & - \\
\hline \multicolumn{2}{|c|}{ Product innovators } & 1909 & 44.3 & 100.0 \\
\hline \multicolumn{5}{|c|}{ Product innovators that introduced: } \\
\hline - & A new to firm product & 1909 & 44.3 & 100.0 \\
\hline - & A new to market product & 1484 & 34.4 & 77.7 \\
\hline - & A first in Québec product & 1180 & 27.4 & 61.8 \\
\hline - & A first in Canada product & 923 & 21.4 & 48.4 \\
\hline - & A first in North America product & 597 & 13.9 & 31.3 \\
\hline - & A world-first product & 251 & 5.8 & 13.2 \\
\hline \multicolumn{2}{|c|}{ Process innovators } & 1423 & 33.0 & 74.6 \\
\hline \multicolumn{5}{|c|}{ Process innovators that introduced: } \\
\hline - & A first in Québec process & 525 & 12.2 & 27.5 \\
\hline - & A first in Canada process & 358 & 8.3 & 18.8 \\
\hline - & A first in North America process & 219 & 5.1 & 11.5 \\
\hline - & A world-first process & 94 & 2.2 & 4.9 \\
\hline \multicolumn{2}{|c|}{ Ongoing innovation activities } & 1610 & 37.4 & 84.3 \\
\hline \multicolumn{2}{|c|}{ Abandoned innovation activities } & 1069 & 24.8 & 56.0 \\
\hline
\end{tabular}

Sources: Statistics Canada, compiled by the Institut de la Statistique du Québec and the author.

Results are summarized in Table 8.3-1. The weighted sample comprised 4310 firms of which, 44.3\% (1 909 firms) declared themselves as product innovators and $33.0 \%$ (1 423 firms) processes innovators. Out of 1,909 product innovators, about $77.7 \%$ (1 484 firms) introduced at least a product innovation that was a new to the firm market, 27.4\% (1 180 firms) a first in Québec and $21.4 \%$ (923 firms) a first in Canada. When considering North America and the World, the proportion of product innovators that introduced a first drops to $13.9 \%$ (597 firms) and 5.8\% ( 251 firms) respectively. Thus, the proportion of product innovators having introduced a first decreases when considering an increasingly large geographic territory. This is also true in the case of process innovation, because, as one can see in Table 8.3-1, the proportion of process innovators varies from $12.2 \%$ (525 firms) in the case of the 
first process in Québec to only $2.2 \%$ (94 firms) for the case of process innovators having introduced a process in the world for the first time. Finally, the questionnaire asks additional question about ongoing and abandoned innovation activities. 1610 firms declared they still had ongoing innovation activities at the end of the reference period and 1069 declared they abandoned innovation activities during the 2002 to 2004 period.

Table 8.3-2: $\quad$ Product innovations distribution following STAN aggregation ${ }^{41}, 2002-2004$

\begin{tabular}{|c|c|c|c|c|c|c|}
\hline & \multirow{2}{*}{\multicolumn{2}{|c|}{$\begin{array}{l}\text { Total population } \\
\text { Number of firms }\end{array}$}} & \multicolumn{4}{|c|}{ Product innovatiors } \\
\hline & & & \multicolumn{2}{|c|}{$\begin{array}{l}\text { Number of } \\
\text { firms }\end{array}$} & \multicolumn{2}{|c|}{$\begin{array}{c}\text { Number of produc } \\
\text { innovations }\end{array}$} \\
\hline & $\mathbf{n}$ & $\%$ & $\mathbf{n}$ & $\%$ & $\mathbf{n}$ & $\%$ \\
\hline Food and Tobacco & 437 & 10.1 & 223 & 11.7 & 6584 & 13.7 \\
\hline Textile Clothing and Leather & 511 & 11.9 & 178 & 9.3 & 14298 & 29.8 \\
\hline Wood products & 473 & 11.0 & 127 & 6.7 & 768 & 1.6 \\
\hline Paper and Printing & 386 & 9.0 & 155 & 8.1 & 2158 & 4.5 \\
\hline $\begin{array}{l}\text { Petroleum Chemical and Plastics \& } \\
\text { Rubber }\end{array}$ & 510 & 11.8 & 265 & 13.9 & 6135 & 12.8 \\
\hline Non-metal products & 163 & 3.8 & 61 & 3.2 & 501 & 1.0 \\
\hline $\begin{array}{l}\text { Primary metal and Fabricated metal } \\
\text { products }\end{array}$ & 621 & 14.4 & 222 & 11.6 & 2609 & 5.4 \\
\hline $\begin{array}{l}\text { Machinery Electrical Electronic computer } \\
\text { and communication }\end{array}$ & 556 & 12.9 & 355 & 18.6 & 6798 & 14.2 \\
\hline Transportation & 152 & 3.5 & 72 & 3.8 & 618 & 1.3 \\
\hline $\begin{array}{l}\text { Furniture and NEC manufacturing } \\
\text { industries }\end{array}$ & 500 & 11.6 & 251 & 13.1 & 7434 & 15.5 \\
\hline Total & 4310 & 100.0 & 1909 & 100.0 & 47903 & 100.0 \\
\hline
\end{tabular}

Sources: Statistics Canada, compiled by the Institut de la Statistique du Québec and the author.

We seek to further characterize the data by disaggregating the samples into different categories, in particular we look, in what follows, at the distribution of product innovators by industry, the innovation rate by geographic localization, size class, and technology level, and the firm's usage of government sponsored programs. Table 8.3-2 shows the distribution of product innovators by industry. As can be seen in the second column of this table, product innovators are distributed fairly evenly across industries with proportions ranging between $9.0 \%$ and $14.4 \%$ excluding the transportation industry and the non-metal industry products, which represent only $3.5 \%$ and $3.8 \%$ respectively in the number of innovative firms. The textile, clothing and leather industry ranks first with 14298 product innovations, or $29.8 \%$ of total product innovations for the three years (2002-2004). It is followed by the furniture and NEC manufacturing industries (15.5\%) and by the machinery,

\footnotetext{
${ }^{41}$ STAN (Structural analysis) is an OECD database for industrial analysis that provides a comprehensive tool for analyzing industrial performance at a relatively detailed level of activity across countries. It is based on the International Standard Industrial Classification of all economic activities (ISIC).
} 
electrical, electronic and computer communication industry (14.2\%). The three industries at the bottom of the ranking are the non-metal products industry, the transportation industry and the wood products industry with $1.0 \%, 1.3 \%$ and $1.6 \%$ of total product innovations.

Breaking firms into technology level (Table 8.3-3) reveals the identity of the innovators: medium-high-technology and high technology firms are more active with rates of innovation close to $60 \%$ (58.7\% and $58.4 \%$ respectively). Furthermore, the distribution of the innovation rate by size class, as detailed in Table 8.3-4, shows that larger firms are more likely to innovate. In this case too, the rate of innovation is around $60 \%$ for firms with 250 to 499 employees and for firms with 500 employees or more.

Table 8.3-3: Innovation rate by technology level ${ }^{42}, 2002-2004$

\begin{tabular}{lcc}
\hline & Number of firms & Innovation rate \\
\hline High-technology & 224 & 58.4 \\
Medium-high-technology & 650 & 58.7 \\
Medium-low-technology & 1127 & 41.0 \\
Low-technology & 2308 & 40.5 \\
\hline Total & $\mathbf{4 3 1 0}$ & $\mathbf{4 4 . 3}$ \\
\hline
\end{tabular}

Sources: Statistics Canada, compiled by the Institut de la Statistique du Québec and the author.

Table 8.3-4: Innovation rate by size class, 2002-2004

\begin{tabular}{lcc}
\hline & Number of firms & Innovation rate \\
\hline$\geq 500$ employees & 100 & 59.0 \\
250 to 499 employees & 200 & 60.4 \\
50 to 249 employees & 1983 & 45.6 \\
20 to 49 employees & 2027 & 40.7 \\
\hline Total & $\mathbf{4 3 1 0}$ & $\mathbf{4 4 . 3}$ \\
\hline
\end{tabular}

Sources: Statistics Canada, compiled by the Institut de la Statistique du Québec and the author.

Previous studies have shown that firms' innovation rate may vary according to geographic location (see for example Jaffe, Trajtenberg and Henderson (1993)). It is assumed that innovators concentrate in large urban areas as they face fewer constraints and have lower costs. In the case of the province of Québec, there are 17 administrative regions that may be aggregated into four economic regions of more or less homogeneous economic intensity. These comprise two metropolitan regions, Capitale-Nationale and Montreal and Laval, and two peripheral regions,

\footnotetext{
42 Based on OECD classification of manufacturing industries using International Standard Industrial Classification (ISIC) rev.3 activity breakdown (see classification in Table 6.D-4, Chapter 6).
} 
that is, Central and Resources regions ${ }^{43}$. As can be seen in Table 8.3-5, the effect of localization is not clear as innovation rates for three regions, namely CapitaleNationale, Montreal and Laval, and Central regions, are not very different. On the other hand, the innovation rate in the Resources regions, which are the regions with the lowest population density and regions remote from metropolitan regions, is clearly lower than that of these last ones.

Table 8.3-5: Innovation rate by geographic localization, 2002-2004

\begin{tabular}{lcc}
\hline & Number of firms & Innovation rate \\
\hline Capitale-Nationale region & 248 & 48.3 \\
Montreal and Laval region & 1428 & 44.8 \\
Central regions & 2144 & 46.1 \\
Resources regions & 490 & 32.8 \\
\hline Total & $\mathbf{4 3 1 0}$ & $\mathbf{4 4 . 3}$ \\
\hline
\end{tabular}

Sources: Statistics Canada, compiled by the Institut de la Statistique du Québec and the author.

Table 8.3-6: $\quad$ Firms usage of government sponsored programs, 2002-2004

\begin{tabular}{|c|c|c|c|c|c|c|c|}
\hline & & \multicolumn{2}{|c|}{$\begin{array}{c}\text { Total } \\
\text { population }\end{array}$} & \multicolumn{2}{|c|}{$\begin{array}{c}\text { Product } \\
\text { innovators }\end{array}$} & \multicolumn{2}{|c|}{$\begin{array}{c}\text { Process } \\
\text { innovators }\end{array}$} \\
\hline & & $\mathbf{n}$ & $\%$ & n & $\%$ & $\mathbf{n}$ & $\%$ \\
\hline \multicolumn{2}{|c|}{ R\&D tax credits: } & 2371 & 55.0 & 1492 & 78.1 & 1140 & 80.1 \\
\hline & Federal government & 2258 & 52.4 & 1434 & 75.1 & 1096 & 77.0 \\
\hline - & Québec government & 1953 & 45.3 & 1248 & 65.4 & 964 & 67.7 \\
\hline \multicolumn{2}{|c|}{ R\&D grants } & 544 & 12.6 & 361 & 18.9 & 272 & 19.1 \\
\hline - & Federal government & 406 & 9.4 & 269 & 14.1 & 205 & 14.4 \\
\hline - & Québec government & 340 & 7.9 & 236 & 12.4 & 186 & 13.1 \\
\hline \multicolumn{2}{|c|}{ Venture capital support } & 175 & 4.1 & 114 & 6.0 & 86 & 6.1 \\
\hline - & Federal government & 74 & 1.7 & 54 & 2.8 & 39 & 2.7 \\
\hline - & Québec government & 116 & 2.7 & 71 & 3.7 & 55 & 3.8 \\
\hline \multicolumn{2}{|c|}{ Technology support and assistance programs } & 352 & 8.2 & 231 & 12.1 & 188 & 13.2 \\
\hline - & Federal government & 191 & 4.4 & 134 & 7.0 & 108 & 7.6 \\
\hline - & Québec government & 231 & 5.4 & 153 & 8.0 & 123 & 8.7 \\
\hline \multicolumn{2}{|l|}{ Total } & 4310 & & 1909 & & 1423 & \\
\hline
\end{tabular}

Sources: Statistics Canada, compiled by the Institut de la Statistique du Québec and the author.

\footnotetext{
${ }^{43}$ See Table 8.C-3 for the classification of Québec's administrative regions into the four economic regions used in this study.
} 
Table 8.3-6 shows firms' usage of government sponsored programs. The last question of the Canadian survey of innovation 2005 asked firms to select the types of programs sponsored by the federal government or provincial governments they used during the three survey years. Government programs to choose from include $R \& D$ tax credits, R\&D grants, venture capital support, technology support and assistance programs.

\subsection{The Model}

In this study, we use two variants of the CDM model that extend the latter in order to capture effects not explicitly accounted for. The first variant is the OECD 2009 core model which is used to compare the Québec results to those of the OECD project. The second variant is an extension of the first variant. In addition to the first variant features, it seeks to capture the role of government incentives by specifying explicitly additional functional equations that explain the effect of government support on firm's R\&D investment and on innovation.

\subsubsection{The OECD Core Model}

The OECD core model has been developed in the framework of the Innovation micro-data project ${ }^{44}$ Phase 2 in which participated research teams from 18 countries ${ }^{45}$. It consists in a system of four equations estimated in three stages as formally presented bellow.

\section{First-stage: Innovation selection equation and innovation input equation}

The first stage is composed of two equations. The first is the innovation selection equation that estimates the probability of firms to engage in innovation activities. The second is the innovation input equation which explains the intensity of innovation expenditures. Formally, the first equation is given by:

$$
\text { innov }_{i}=\left\{\begin{array}{l}
1 \text { if innov }{ }_{i}=x_{2 i} b_{2}+\mu_{2 i}>0 \\
0 \text { if innov }{ }_{i}=x_{2 i} b_{2}+\mu_{2 i} \leq 0
\end{array}\right.
$$

where $\mu_{2 i} \sim N(0,1)$, innov $v_{i}$ is a binary variable that takes the value 1 if a firm has positive innovation expenditures, and 0 otherwise. innov ${ }_{i}{ }_{i}$ is the unobservable

\footnotetext{
${ }^{44}$ The OECD Innovation micro-data project was first launched in 2006.

45 Australia, Austria, Belgium, Brazil, Canada, Denmark, Finland, France, Germany, Italy, Korea, Luxembourg, Netherlands, New Zealand, Norway, Sweden, Switzerland, United Kingdom.
} 
counterpart of innov $v_{i}, X_{2 i}$ is a vector of variable that explains the decision of the firm to engage in innovating activities or not and $b_{2}$ is an unknown parameters to be estimated.

The second equation is given by:

Irtotpe $_{i}=X_{3 i} b_{3}+\mu_{3 i}$ if innov in $_{i} 1$

where $\mu_{3 i} \sim N\left(0, \sigma_{3}\right)$ and, by construction, $\mu_{2}$ is correlated to $\mu_{3}$ so that $\operatorname{Corr}\left(\mu_{2 i}, \mu_{3 i}\right)=\rho_{23}$. Irtotpe ${ }_{i}$ represents the input of innovation which is proxied by the ratio of innovation expenditures to the number of employees. $X_{3 i}$ is a vector of variables that explains innovation input (see Section 8.5.3) for a more detailed description). $b_{3}$ is the parameter to be estimated.

Equations (8-1) and (8-2) are modeled as a generalized Tobit model that explains simultaneously the probability for a firm to engage in innovating activities and the intensity of the firm's innovation input. The two equations are estimated using the Heckman's sample selection model, first introduced by James Heckman (Heckman (1979)). The Heckman's selection model permits to correct for sample selection bias that could arise if the sample of innovators was used in a simple OLS regression. It leads to the computation of the Inverse Mills Ratio (IMR) which is used in the subsequent equations to take into account the sample selection bias.

\section{Second stage: Innovation output equation}

The innovation output equation which is the third equation represents the output side of innovation in the model and is given by:

$$
\text { lispe }_{i}=X_{4 i} b_{4}+\overline{\text { Irtotpe }_{i}}+\text { Mill }_{3 i} b_{m r}+\mu_{4 i} \text { if innov } i=1
$$

where lispe $_{i}$ represents the ratio of innovative sales to the number of employees, $X_{4 i}$ is a vector of variables that explains innovation output (see Section 8.5.3 for a more detailed description). $b_{4}$ and $b_{m r}$ are the parameters to be estimated. $\overline{\text { Irtotpe }_{i}}$ is the predicted value of Irtotpe ${ }_{i}$ estimated in the previous equation, (Equation (8-2)). The variableMill ${ }_{3 i}$ is the IMR constructed in the first stage. The term $\mu_{4}$ is an i.i.d. disturbance that follows a normal distribution with zero mean and variance such that, $\mu_{4 i} \sim N\left(0, \sigma_{4}\right)$. 


\section{Third-stage: Productivity equation}

The model's fourth equation estimates a measure of the firm's performance such that:

$$
\text { Ivape }_{i}=X_{5 i} b_{5 i}+\overline{\text { lispe }_{i}}+\text { Mill }_{3 i} b_{m r}+\mu_{5 i} \text { if innov } i
$$

where Ivape $_{i}$ is the productivity of the firm proxied by the log of value added per employee (or alternatively by the log of sales per employee). $X_{5 i}$ is a vector of independent variables that explain the firm's productivity, $\overline{l i s p e_{i}}$ is the predicted value of lispe $_{i}$, the ratio of innovative sales to the number of employees, and finally the disturbance $\mu_{5 i} \sim \mathrm{N}\left(0, \sigma_{5}\right)$.

\subsubsection{Extending the OECD Model: The Québec Model}

As mentioned in the introduction, this study emphasizes the role of government incentives in firms' decision to innovate. Hence, relying on the richness of the data set constructed by linking the data from the Innovation survey 2005 to administrative data on R\&D (see Section 5 for a detailed description of the dataset), we could add to the OECD model additional functional equations that explain the effect of government support on firm's R\&D investment and on innovation.

In the first stage, the core model of the OECD is modified and extended to take account of the determinants of government support for innovation. Following González, Jaumandreu and Pazó (2005) and Garcia and Mohnen (2010), we do this by estimating in the first stage, prior to modeling firms' decision to innovate (i.e., Equation (8-1)), an equation that model firms' expectations about government funding for innovation. We also estimate along that equation, another equation that takes account of the intensity of government support.

These two equations also are modeled as a generalized Tobit model as in the case of Equations (8-1) and (8-2) since all firms in the sample are not government support recipients. In this case, the generalized Tobit model permits to explain simultaneously the probability for a firm to claim government support as well as the intensity of the government support. In sum, the extended model for Québec is build by transforming the four-equation OECD model into a six-equation model. Since, the two additional equations are estimated prior to the estimation of the first equation in the OECD model, only the first stage is now modified to contain four equations rather than two. 


\section{Modified first-stage: Selection equation for government support, government support equation and innovation selection equation}

The two additional equations, i.e. the selection equation for government support and the government support equation respectively denoted recip $p_{i}$ and Igovsup pe $e_{i}$ are given by:

$$
\operatorname{recip}_{i}=\left\{\begin{array}{l}
1 \text { if recip }{ }_{i}=X_{0 i} b_{0}+\mu_{0 i}>0 \\
0 \text { if recip }{ }_{i}=X_{0 i} b_{0}+\mu_{0 i} \leq 0
\end{array}\right.
$$

Igovsuppe $_{i}=X_{1 i} b_{1}+\mu_{1 i}$ if recip r $_{i}=1$

where recip $_{i}$ is a binary variable that takes the value 1 if a firm receives any type of government support, and 0 otherwise. recip ${ }_{i}{ }_{i}$ is the latent variable that generates the observed state in the firm's decision variable recip $p_{i}$, i.e. whether to apply for government support or not. Igovsuppe $e_{i}$ represents the amount of government support per employee. $X_{0 i}$ is a vector of variables that explain the decision of the firm to apply for governmental support or not, and $X_{1 i}$ is a vector of variables that explain the intensity of the government support. The variables in vectors $X_{0 i}-X_{1 i}$ are described in more details in Section 8.5.3. $b_{0}$ and $b_{1}$, are the unknown parameters to be estimated. Finally, the disturbance terms are distributed such that $\mu_{0 i} \sim N(0,1)$ and $\mu_{1 i} \sim N\left(0, \sigma_{1}\right)$ and $\operatorname{corr}\left(\mu_{0 i}, \mu_{1 i}\right)=\rho_{01}$.

Equation (8-1) is modified to include $\overline{\text { Igovsuppe }}_{i}$, the predicted value of Igovsuppe $e_{i}$ computed from Equations (8-5) and (8-6):

$$
\text { innov }_{i}=\left\{\begin{array}{l}
1 \text { if innov }{ }_{i}=x_{2 i} b_{2}+\overline{\overline{\operatorname{lgovsup}}_{i}}+\mu_{2 i}>0 \\
0 \text { if innov }{ }_{i}=x_{2 i} b_{2}+\overline{\operatorname{lgovsup} p e_{i}}+\mu_{2 i} \leq 0
\end{array}\right.
$$

Similarly, Equation (8-2) is also modified to include the IMR computed from Equations (8-5) and (8-6):

$$
\text { Irtotpe }_{i}=X_{3 i} b_{3}+\text { Mill }_{1 i} b_{m r}+\mu_{3 i} \text { if innov } i
$$

The rest of the model remains unchanged. Figure 8.4-1 compares the OECD core model to the extended model for Québec. 
Figure 8.4-1: The OECD core model and the extended model for Québec

EXTENDED MODEL FOR QUÉBEC

OEDC CORE MODEL
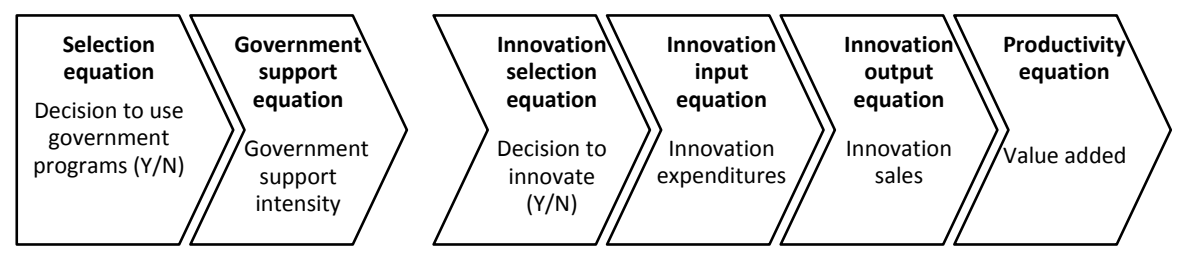

First stage

$\begin{array}{ll}\text { I Second } & \text { I Third stage } \\ \text { I stage } & \text { I }\end{array}$

\subsection{Data and Variables}

\subsubsection{Data}

The data are sourced from four microdata files, namely the IS2005, the ASM, the RCDI and the ADRQ. From these microdata, we constructed two datafiles: the IS2005-ASM matched data ${ }^{46}$ for the estimation of the OECD core model and the IS2005-ASM-RCDI-ADRQ matched data for the estimation of the Québec extended model. The final sample for the IS2005-ASM matched data has 2747 observations and the IS2005-ASM-RCDI-ADRQ data is constituted of 2166 observations.

The four microdata sources and the matching process are described in details in Section 4.3.3.

\subsubsection{Correspondance CIS 4 - IS2005}

The data of OECD countries used for the estimation of the OECD model come from the fourth Community Innovation Statistics (CIS 4) which was produced in 27 Member States of the European Union, 3 countries of the European Free Trade Association (EFTA). Both the CIS 4 and the Statistics Canada Survey of Innovation 2005 are based on the second edition of the Oslo Manual (OECD (1997)) which gives methodological guidelines and defines the innovation concept. In addition, they were implemented based on the reference years 2002/2004. For these reasons, data gathered through these surveys may be used for comparative purposes like in the present study. However, despite the harmonization effort, there are differences between the two surveys. The IS2005 questionnaire contains a number of questions

\footnotetext{
${ }^{46}$ Matching of the IS2005 to the ASM data was necessary because the IS2005 alone lacks a number of variables necessary for the estimation of the model such as the number of employees, turnovers and value added.
} 
that are not part of the harmonised CIS 4 and vice versa. Table 8.5-1 shows the correspondence between both surveys. Survey questionnaires are presented in full in the appendix to this chapter (Appendix 8.D for IS2005 and Appendix 8.E for CIS 4). We added variable names used in this chapter which are identical to those used in the OECD 2009 study.

Table 8.5-1: Correspondance between CIS 4 and IS2005

\begin{tabular}{|c|c|}
\hline Fourth Community Innovation Survey (CIS 4) & Survey of Innovation 2005 (IS2005) \\
\hline Q1.1 & Q1 \\
\hline Q1.2 & Q1 \\
\hline Q2.1 & Q9 \\
\hline Q2.2 & Q11 \\
\hline Q2.3 & Q12-Q13 \\
\hline Q3.1 & Q17 \\
\hline Q3.2 & Q18 \\
\hline Q4.1 & Q20-Q21 \\
\hline Q5.1 & Q23 \\
\hline Q5.2 & - \\
\hline Q5.3 & Q41 \\
\hline Q6.1 & Q25 \\
\hline Q6.2 & Q26 \\
\hline Q6.3 & Q27 \\
\hline Q6.4 & Q28 \\
\hline Q7.1 & Q29 \\
\hline Q.8.1 & - \\
\hline Q8.2 & Q30 \\
\hline Q9.1 & Q31 \\
\hline Q10 & - \\
\hline Q11 & - \\
\hline
\end{tabular}

Here are the main differences between the CIS 4 and the IS2005:

1. The respondent unit is the enterprise in the case of CIS 4 while it is the establishment in the case of the IS2005.

2. In the CIS 4 questionnaire, questions 5.1 to 8.1 were asked to enterprises that had product or process innovations or innovation activities during 2002 to 200 and those that have product or process innovations that were abandoned during this period. In the IS2005 questionnaire, corresponding questions are asked only to establishments that have product or process innovation between 2002 and 2004.

3. In Question 5.1 of the CIS 4, enterprises were asked if the "R\&D was performed continuously or occasionally during 2002 to 2004. The Canadian survey did not ask to make such difference. 
4. Question 5.2 of CIS 4 has no counterpart in the Canadian survey (the latter did not ask establishments to estimate the amount of expenditures devoted to intramural $R \& D$, to extramural $R \& D$, to the acquisition of machinery, equipment and software, and to the acquisition of other external knowledge).

5. Question 8.1 of CIS 4 has no counterpart in the Canadian survey (the latter did not ask establishment if during the three years, 2002 to 2004, if any innovation activities or projects were abandoned in the concept stage, after the activity or project was begun, or seriously delayed).

6. In the CIS 4 survey, Question 8.2 (barriers to innovation) is asked to all enterprises. In the Canadian survey, the corresponding question (30) is asked only to establishments that have innovated between 2002 and 2004.

8. Question 10 (organisational and marketing innovations) and Question 11 (economic information of the enterprise such as enterprise's total turnover and number of employees during the reference period) of CIS 4 have no equivalent in the Canadian survey.

As it will be noticed in the following sections, in the data processing and interpretation of results, these differences are taken into account.

\subsubsection{Variables}

This section describes the vectors of independent variables $X_{0 i}-X_{5 i}$ presented in Section 8.4. It should be noted that these refer only to the vectors of variables included in the extended model for Québec which uses a set of variables richer than the one used in the OECD core model. However, Table 8.5-2 presents the complete list of variables included in the OECD core model and in the extended model. Finally, variables construction and sample descriptive statistics for the extended model of Québec are presented in Table 8.5-3.

\subsubsection{First-Stage Variables}

\section{Variables of the selection and the government support equations}

$$
\begin{aligned}
& X_{0 i}=\left(l e m p_{i}, l_{e m p_{i}^{2}}^{2}, r d_{-} \text {pers } s_{i}, m k t_{-} s h r_{i}, c r 4_{i}, \text { export_ot }{ }_{i},\right. \\
& \text { export_us }{ }_{i}, r d \_o u t_{i}, \text { sic }_{i}, \text { geo }_{i} \text { ) }
\end{aligned}
$$

and

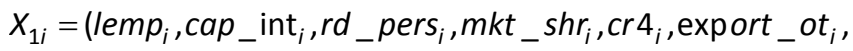

$$
\begin{aligned}
& \text { export_us }{ }_{i}, r d \_o u t_{i}, \text { sic }_{i}, g o_{i} \text { ) }
\end{aligned}
$$


where lemp is the log of employment and rd_pers is the firm's share of employees involved in R\&D activities. To take account of variables that control for market competition, we included four variables: market share (mkt_shr), a concentration ratio (cr_4) and export_ot and export_us, respectively, the percentage of the firm's total revenue that comes from sales outside Canada and the US and the percentage of the firm's total revenue that comes from the US. Another covariate added in the equation is rd_out a dummy variable indicating if the firm has contracted-out for R\&D services. Industrial dummies, sic, are also added to take account of the heterogeneity among industries. Also, we take account of the localization effect by including geographic dummies into the model ( $g e o$ ). Specially, these dummies are included in the first four equations of the model (first stage equations) as the localization of the firm can affect its probability of applying for public funds and that of engaging in research and innovation activities. Indeed, this probability is expected to be higher for firms located in the major economic regions such as the Montreal and Laval urbain communities or the National Capital region because of the concentration of research activities and of regional incentives in these regions. Concerning this last point, one can cite for example the indirect measures established by the Government of Québec in the late 1990s and early 2000s for firms carrying out innovation activities in certain designated sites, i.e. CDTIs, CMM, CNNTQ, CNEs, CCE and CDBs (see Section 3.3.1).

In the selection Equation (8-7), in addition to lemp, the square of the latter is included to help control for size effects arising from the potential endogeneity between the receipt of public funding and the hiring of R\&D personnel. In the government support Equation (8-8), cap_int, the firm's capital intensity, measured by the ratio of total asset per employee, is added to capture the influence of capital structure on the intensity of R\&D activities. In addition, the level of a firm's total asset influences the percentage of fiscal relief given by the government.

\section{Variables of the innovation selection equation}

$$
\begin{aligned}
& X_{2 i}=\left(l e m p_{i}, \text { export_ot }_{i}, \text { export_us }{ }_{i}, f a c_{-} \text {new }_{i}, \text { fac_custom }_{i}\right. \text {, } \\
& f a c_{-} d e v_{i}, m k t \_s h r_{i}, c r 4_{i}, m_{-} e_{i}, \text { method_inst }{ }_{i}, \text { method_priv } v_{i} \text {, } \\
& \text { method_stra } i, \mathrm{sic}_{i}, \mathrm{geo}_{i} \text { ) }
\end{aligned}
$$

In the innovation selection equation, three important success factors for the firm are included: fac_new, seeking new markets, fac_custom, developing customdesign products for clients and fac_dev developing niche, specialized markets, export markets or domestic markets. We also included three dummy variables that indicate what types of methods firms use to protect their intellectual property: method_inst institutional methods (patents, trademarks or copyrights), 
method_priv private methods (confidentiality agreements or secrecy) and method_stra strategic methods (complexity of design or lead-time advantage on competitors).

\section{Variables of the innovation input equation}

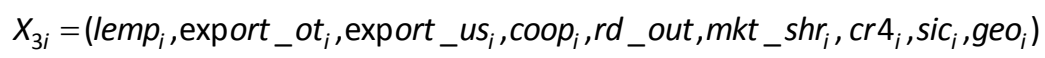

The variable coop, an indicator for co-operation on innovation activities between the firm and other firms or institutions, is included to test the hypothesis that co-operation is correlated with innovation.

\subsubsection{Second-Stage Variables: Innovation Output Equation}

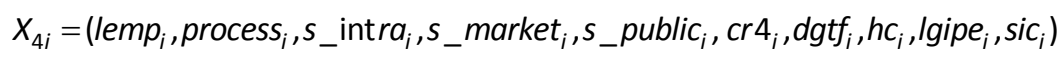

In the innovation output equation, process a dummy indicating the introduction of a process innovation, is added to assess its effects on innovation sales. We included three types of information for the firm's innovation activities. These types of sources are s_intra, internal sources (R\&D, sales and marketing, production or management staff), s_market, market sources (suppliers, clients or customers, competitors, consultants, commercial labs or R\&D firms) and s_public, institutional sources (universities, colleges, technical institutes or government or private nonprofit research laboratories). Also included in the equation is the distance from the technology frontier, dgtf, which is the difference between the firm's productivity level and the productivity level of the top global firms at the $90^{\text {th }}$ percentile. The frontier has been computed by the OECD team by pooling firms from all participating countries and the US using Compustat for Canadian and US firms and Orbis/Amadeus for firms from Europe and Asia. Finally, we also experimented with capital intensity and (Igipe) and human capital (hc).

\subsubsection{Third-Stage Variables: Productivity Equation}

$$
X_{5 i}=\left(\text { lemp }_{i}, \text { process }_{i}, \mathrm{cr}_{i}, \text { dgtf }_{i}, h c_{i}, \text { Igipe }_{i}, \text { sic }_{i}\right)
$$

As it appears in Equation (8-12), process is also included among covariates in order to allow the estimation of the contribution of process innovation to labour productivity along with that of product innovation to labour productivity. 
Table 8.5-2: Causality of the OECD core model and the extended model for Québec

\begin{tabular}{|c|c|c|c|c|c|c|}
\hline & \multicolumn{4}{|c|}{ First stage } & $\begin{array}{l}\text { Second } \\
\text { stage }\end{array}$ & Third stage \\
\hline & \multicolumn{6}{|c|}{ Extended model for Québec } \\
\hline & & & \multicolumn{4}{|c|}{ OECD model } \\
\hline & $\begin{array}{c}\text { Government } \\
\text { support } \\
\text { selec-tion } \\
\text { equation }\end{array}$ & $\begin{array}{l}\text { Government } \\
\text { support } \\
\text { equation }\end{array}$ & $\begin{array}{l}\text { Innovation } \\
\text { selection } \\
\text { equation }\end{array}$ & $\begin{array}{l}\text { Innovation } \\
\text { input } \\
\text { equation }\end{array}$ & $\begin{array}{l}\text { Innovation } \\
\text { output } \\
\text { equation }\end{array}$ & $\begin{array}{c}\text { Productivity } \\
\text { equation }\end{array}$ \\
\hline $\begin{array}{c}\text { Dependant } \\
\text { variable }\end{array}$ & recip & Igovsuppe & innov & Irtotpe & lispe & Ivape (IIpe) \\
\hline \multicolumn{7}{|l|}{ Regressors } \\
\hline$g p$ & & & $\square$ & $\square$ & $\square$ & $\square$ \\
\hline lemp & $\bullet$ & $\bullet$ & $\mathbf{x}$ & $\bullet$ & $\mathbf{x}$ & $\mathbf{x}$ \\
\hline lemp2 & $\bullet$ & & & & & \\
\hline cap_int & & $\bullet$ & & & & \\
\hline rd_pers & $\bullet$ & $\bullet$ & & & & \\
\hline$m k t \_s h r$ & $\bullet$ & $\bullet$ & $\bullet$ & $\bullet$ & & \\
\hline export_ot & $\bullet$ & $\bullet$ & $\bullet$ & $\bullet$ & & \\
\hline export_us & $\bullet$ & $\bullet$ & $\bullet$ & $\bullet$ & & \\
\hline for_mkt & & & $\square$ & $\square$ & & \\
\hline haknow & & & $\square$ & & & \\
\hline hamarket & & & $\square$ & & & \\
\hline hacost & & & $\square$ & & & \\
\hline finsup & & & $\square$ & & & \\
\hline fac_new & & & $\bullet$ & & & \\
\hline fac_custom & & & $\bullet$ & & & \\
\hline fac_dev & & & $\bullet$ & & & \\
\hline coop & & & & $x$ & & \\
\hline coop_client & & & & & $\square$ & \\
\hline coop_supplier & & & & & $\square$ & \\
\hline coop_private & & & & & $\square$ & \\
\hline coop_public & & & & & $\square$ & \\
\hline rd_out & $\bullet$ & $\bullet$ & & $\bullet$ & & \\
\hline process & & & & & $x$ & $x$ \\
\hline s_intra & & & & & $\bullet$ & \\
\hline s_market & & & & & $\bullet$ & \\
\hline s_public & & & & & $\bullet$ & \\
\hline$h c$ & & & & & $\bullet$ & $\bullet$ \\
\hline lgipe & & & & & $\bullet$ & $\bullet$ \\
\hline $\mathrm{cr} 4$ & $\bullet$ & $\bullet$ & $\bullet$ & $\bullet$ & $\bullet$ & \\
\hline$d g t f$ & & & & & $\bullet$ & $\bullet$ \\
\hline m_e & & & $\bullet$ & & & \\
\hline method_inst & & & $\bullet$ & & & \\
\hline method_priv & & & $\bullet$ & & & \\
\hline method_stra & & & $\bullet$ & & & \\
\hline sic & $\bullet$ & $\bullet$ & $x$ & $x$ & $x$ & $x$ \\
\hline geo & $\bullet$ & $\bullet$ & $\bullet$ & $\bullet$ & & \\
\hline Mill $_{1}$ & & & & $\bullet$ & & \\
\hline $\mathrm{Mill}_{3}$ & & & & & $x$ & $x$ \\
\hline Igovsuppe & & & $\bullet$ & $\bullet$ & & \\
\hline Irtotpe & & & & & $x$ & \\
\hline lispe & & & & & & $x$ \\
\hline \multirow[t]{3}{*}{ Legend: } & $\bullet$ & \multirow{3}{*}{\multicolumn{5}{|c|}{$\begin{array}{l}\text { Only in the extended model for Québec } \\
\text { Only in the OECD model } \\
\text { In both the extended model for Québec a }\end{array}$}} \\
\hline & $\square$ & & & & & \\
\hline & $x$ & & & & & \\
\hline
\end{tabular}


Table 8.5-3: Variables construction and sample descriptive statistics, all firms

\begin{tabular}{|c|c|c|c|}
\hline Variable & Description & Mean & S.D. \\
\hline$g p$ & Dummy for belonging to a group, 1=if firm belongs to a group & 0.19 & 0.39 \\
\hline lemp & Log of employment, proxy for the firm's size & 3.72 & 0.90 \\
\hline lemp2 & Square of log employment & 14.61 & 7.36 \\
\hline cap_int & Capital intensity (firm's real assets per employee) & 11.49 & 0.74 \\
\hline Igipe & Log capital intensity & 11.64 & 0.69 \\
\hline rd_pers & Percentage of full time employees involved in R\&D activities & 8.98 & 13.55 \\
\hline$m k t \_s h r$ & $\begin{array}{l}\text { Market share, constructed as firm's real sales divided by industry's } \\
\text { real sales (NACE } 4 \text { digits level) }\end{array}$ & 0.02 & 0.05 \\
\hline export_ot & Firm's percentage of sales exported outside Canada and the US & 0.04 & 0.12 \\
\hline export_us & Firm's percentage of sales exported to the US & 0.21 & 0.27 \\
\hline for_mkt & $\begin{array}{l}\text { Dummy for presence in international markets, } 1=\text { if firm is active in } \\
\text { foreign markets }\end{array}$ & 0.69 & 0.46 \\
\hline haknow & $\begin{array}{l}\text { Dummy for obstacles to innovation, } 1=\text { if firm is influenced by know- } \\
\text { ledge factors (lack of, qualified personnel, information on technology } \\
\text { or on market; difficulty to find cooperation partners for innovation) }\end{array}$ & na & na \\
\hline hamarket & $\begin{array}{l}\text { Dummy for obstacles to innovation, } 1=\text { if firm is influenced by market } \\
\text { factors (market dominated by established enterprises, uncertain } \\
\text { demand for innovative goods or services) }\end{array}$ & na & na \\
\hline hacost & $\begin{array}{l}\text { Dummy for obstacles to innovation, } 1=\text { if firm is influenced by cost } \\
\text { factors (lack of fund, innovation costs too high) }\end{array}$ & na & na \\
\hline fac_new & $\begin{array}{l}\text { Dummy for success factors, } 1=\text { if seeking new markets is highly } \\
\text { important for the firm }\end{array}$ & 0.33 & 0.47 \\
\hline fac_custom & $\begin{array}{l}\text { Dummy for success factors: } 1=\text { if developing custom-designed } \\
\text { products (good or services) for clients is highly important for the firm }\end{array}$ & 0.47 & 0.50 \\
\hline fac_dev & $\begin{array}{l}\text { Dummy for success factors, } 1=\text { if developing niche, specialized } \\
\text { markets, export markets or domestic markets is highly important for } \\
\text { the firm }\end{array}$ & 0.53 & 0.50 \\
\hline coop & $\begin{array}{l}\text { Dummy for co-operation, } 1=\text { if firm co-operated with other firms or } \\
\text { organizations on innovation activities }\end{array}$ & 0.13 & 0.34 \\
\hline rd_out & $\begin{array}{l}\text { Dummy for contracting out R\&D services, } 1=\text { if firm contracted out for } \\
\text { R\&D services }\end{array}$ & 0.15 & 0.35 \\
\hline process & $\begin{array}{l}\text { Dummy for process innovation, } 1=\text { if firm introduced a new or } \\
\text { significantly improved production process, distribution method, or } \\
\text { support activity for its goods or services }\end{array}$ & 0.54 & 0.50 \\
\hline s_intra & $\begin{array}{l}\text { Dummy for sources of information, } 1=\text { if internal sources of } \\
\text { information is highly important for firm' innovation activities }\end{array}$ & 0.54 & 0.50 \\
\hline s_market & $\begin{array}{l}\text { Dummy for sources of information, } 1=\text { if market sources of } \\
\text { information is highly important for firm' innovation activities }\end{array}$ & 0.43 & 0.49 \\
\hline s_public & $\begin{array}{l}\text { Dummy for sources of information, } 1=\text { if public sources of information } \\
\text { is highly important for firm' innovation activities }\end{array}$ & 0.04 & 0.19 \\
\hline$h c$ & $\begin{array}{l}\text { Human capital (percentage of full time employees with university } \\
\text { degree) }\end{array}$ & 0.07 & 0.11 \\
\hline cr4 & $\begin{array}{l}\text { Concentration ratio, constructed as the revenue of the four largest } \\
\text { firms divided by the industry's total revenue (NACE } 4 \text { digits level) }\end{array}$ & 0.34 & 0.20 \\
\hline dgtf & $\begin{array}{l}\text { Global technology frontier (distance to top firm productivity level } \\
\text { from } 18 \text { countries) }\end{array}$ & 50.45 & 34.90 \\
\hline m_e & $\begin{array}{l}\text { Dummy for machinery and equipment, } 1=\text { if firm bought new } \\
\text { machinery and equipment }\end{array}$ & 0.60 & 0.49 \\
\hline method_inst & $\begin{array}{l}\text { Dummy for intellectual property protection method, 1=if firm used } \\
\text { patents, trademarks or copyrights }\end{array}$ & 0.38 & 0.49 \\
\hline method_priv & $\begin{array}{l}\text { Dummy for intellectual property protection method, 1=if firm used } \\
\text { confidentiality agreements or secrecy }\end{array}$ & 0.46 & 0.50 \\
\hline
\end{tabular}




\begin{tabular}{|c|c|c|c|}
\hline Variable & Description & Mean & S.D. \\
\hline method_stra & $\begin{array}{l}\text { Dummy for intellectual property protection method, } 1=\text { if firm used } \\
\text { complexity of design or lead-time advantage on competitors }\end{array}$ & 0.49 & 0.50 \\
\hline recip(finsup) & $\begin{array}{l}\text { Dummy for government support recipient, } 1=\text { if firm received public } \\
\text { financial support }\end{array}$ & 0.35 & 0.48 \\
\hline Igovsuppe & Log of amount of government support per employee & 7.10 & 1.37 \\
\hline innov & Dummy for innovator, $1=$ if firm has positive innovation expenditures & 0.67 & 0.47 \\
\hline Irtotpe & Log of total innovation expenditures per employee & 9.04 & 1.17 \\
\hline lispe & Log of innovation sales per employee & 10.01 & 1.13 \\
\hline Ivape & Log of value added per employee & 11.14 & 0.53 \\
\hline Ilppe & Log of labour productivity per employee & 12.02 & 0.56 \\
\hline \multicolumn{4}{|c|}{ Industrial dummies } \\
\hline Sic1 & 1.Food and Tobacco & 0.08 & 0.26 \\
\hline Sic2 & 2.Textile, Clothing and Leather & 0.13 & 0.34 \\
\hline Sic3 & 3. Wood products & 0.11 & 0.31 \\
\hline Sic4 & 4.Paper and Printing & 0.08 & 0.28 \\
\hline Sic5 & 5.Petroleum, Chemical and Plastics \& Rubber & 0.10 & 0.30 \\
\hline Sic6 & 6.Non-metal products & 0.03 & 0.17 \\
\hline Sic7 & 7.Primary metal and Fabricated metal products & 0.16 & 0.37 \\
\hline Sic8 & 8.Machinery, Electrical, Electronic computer and communication & 0.15 & 0.36 \\
\hline Sic9 & 9.Transportation & 0.03 & 0.17 \\
\hline Sic10 & 10.Furniture and NEC manufacturing industries & 0.13 & 0.33 \\
\hline \multicolumn{4}{|c|}{ Geographic dummies } \\
\hline Geo1 & 1.Central regions & 0.50 & 0.50 \\
\hline Geo2 & 2.Resources regions & 0.09 & 0.29 \\
\hline Geo3 & 3.National Capital region & 0.06 & 0.23 \\
\hline Geo4 & 4.Montreal and Laval region & 0.35 & 0.48 \\
\hline $\begin{array}{l}\text { Number of } \\
\text { observations }\end{array}$ & 2166 & & \\
\hline
\end{tabular}

Sources: Revenu Québec, Statistics Canada, compiled by the Institut de la Statistique du Québec and the authors.

\subsection{Results}

\subsubsection{Results of the Québec Extended Model}

\subsubsection{Empirical Analysis}

We estimated a CDM type model which, by adding an explicit function of government support, allows assessing the effects of public incentives on innovation and productivity. This section describes the samples used and how we handled keys hurdles and challenges in parameter estimation of the model. The results for the extended model for Québec are presented in the next section.

Table 8.6-1 presents the samples used in the estimation of the model. Our CDM model has a more detailed structure capable of handling the rich data available to us, but this is not without cost: as we move forward in the estimation, we lose a significant number of observations. Thus, the structure of the model is such that, for 
the first two equations of the model (Equations (8-5) and (8-6)), the total sample composed of 2166 observations is used. For the third equation and fourth equation (Equations (8-7) and (8-2)), the sample reduces to the sample of recipients firms which comprises a total of 765 observations. Finally, for the fifth and sixth equations (Equations (8-3) and (8-4)), only recipients firms with positive innovation expenditures and innovation sales are left in the estimation sample (501 observations $)^{47}$. This sample is defined as the innovative firms' sample in both the extended and the OECD models. The choice of the innovation definition used in our model was made so as to be consistent with the study of OECD (2009). However, in our case, it should be noted that there is almost no difference in the estimation results whether we use this definition or a wider definition in which is considered as innovative firm, the ones with positive innovation expenditures. As shown in Table 8.6-1, it is merely due to the relatively small gap between the number of observations with positive innovation expenditures (659) and the number of observations with positive innovation expenditure and sales (501).

Table 8.6-1: Description of the samples for the Québec extended model

\begin{tabular}{|c|c|c|c|c|c|c|c|}
\hline \multicolumn{4}{|c|}{ Total sample (n) } & \multicolumn{4}{|c|}{ Total sample (\%) } \\
\hline \multirow[b]{2}{*}{ Innovation expenditures>0 } & \multicolumn{3}{|c|}{ Innovation sales>0 } & \multirow[b]{2}{*}{ Innovation expenditures $>0$} & \multicolumn{3}{|c|}{ Innovation sales>0 } \\
\hline & No & Yes & Total & & No & Yes & Total \\
\hline No & 691 & 22 & 713 & No & 58.3 & 2.2 & 32.9 \\
\hline Yes & 495 & 958 & 1453 & Yes & 41.7 & 97.8 & 67.1 \\
\hline Total & 1186 & 980 & 2166 & Total & 100.0 & 100.0 & 100.0 \\
\hline \multicolumn{4}{|c|}{ Recipients sample (n) } & \multicolumn{4}{|c|}{ Recipients sample (\%) } \\
\hline & \multicolumn{3}{|c|}{ Innovation sales >0 } & & \multicolumn{3}{|c|}{ Innovation sales>0 } \\
\hline Innovation expenditures >0 & No & Yes & Total & Innovation expenditures $>0$ & No & Yes & Total \\
\hline No & 100 & 6 & 106 & No & 38.8 & 1.2 & 13.9 \\
\hline Yes & 158 & 501 & 659 & Yes & 61.2 & 98.8 & 86.1 \\
\hline Total & 258 & 507 & 765 & Total & 100.0 & 100.0 & 100.0 \\
\hline \multicolumn{4}{|c|}{ Non-recipients sample (n) } & \multicolumn{4}{|c|}{ Non-recipients sample (\%) } \\
\hline & \multicolumn{3}{|c|}{ Innovation sales>0 } & & \multicolumn{3}{|c|}{ Innovation sales>0 } \\
\hline Innovation expenditures >0 & No & Yes & Total & Innovation expenditures $>0$ & No & Yes & Total \\
\hline No & 591 & 16 & 607 & No & 63.7 & 3.4 & 43.3 \\
\hline Yes & 337 & 457 & 794 & Yes & 36.3 & 96.6 & 56.7 \\
\hline Total & 928 & 473 & 1401 & Total & 100.0 & 100.0 & 100.0 \\
\hline
\end{tabular}

\footnotetext{
${ }^{47}$ To minimize the loss of observations, another possibility would be to use the full sample again in the second stage rather than the sample of subsidized firms from the first step. This possibility could not be explored in depth because of restrictions on access to data.
} 
Several estimation techniques have been used in order to correct for potential biases such as selectivity or simultaneity bias. For the first four equations forming two Heckman selection models, the estimation is done using the fullinformation maximum likelihood estimator (MLE). The fifth equation (innovation output) and sixth equation (productivity) are estimated by two-stage least squares (2SLS) instrumental variables (IV) as explained further below.

In the estimation process of our model, the first main concern is the validity of each of the two Heckman selection models used to correct the potential selection bias that may arise respectively from: i) in the first case, using the total sample while only $35.3 \%$ of the observations received government support; ii) in the second case, using the total sample of recipients while only $98.8 \%$ of the observations pertain to innovators. To validate the use of the Heckman's selection model, we tested the statistical significance of both rho's $\left(\rho^{\prime}\right.$ and $\left.\rho^{\prime \prime}\right)$. The likelihood ratio test of rho $=0$ in the first case reported a chi2 of 2.78 with a P-value of 0.0955 and, in the second case, a chi2 of 2.98 with a P-value of 0.0843 . This provides evidence that in both cases rho is different from zero and that sample selection bias cannot safely be ignored. In other words, the statistically significant coefficient of the rho's legitimates the use of the double Heckman's sample selection models.

Another main concern is the question of potential endogeneity mentioned in Section 8.4. To address this question, we investigated whether the following couple of variables entering the model should be considered as endogenous: first, government support (Igovsuppe) in innovation decision (innovation selection equation, i.e. innov equation), second, innovation input (Irtotpe) in innovation output (innovation output equation), and third, innovation output in productivity (productivity equation). To check for the triple endogeneity, we performed, as suggested by Davidson and MacKinnon (1993), the Durbin-Wu-Hausman augmented regression test (DWH test). In both the case of government support and innovation decision and in the case of innovation input and innovation output, we fail to reject the null hypothesis of the DWH test, suggesting that endogeneity is present. However, we could not find evidence of endogeneity in the case of innovation output and productivity. In order to deal with endogeneity, some corrections are applied. Specifically, in the innovation selection equation, the endogenous variable, i.e. government support variable (Igovsuppe), is replaced by its predicted value computed from the previous model of Heckman. In the innovation output equation, the innovation input variable (Irtotpe) is instrumented and the equation is estimated by 2SLS instrumental variables so as to provide consistent estimates of the structural parameter. Finally, for the last case concerning the productivity equation, even if endogeneity is rejected, a correction is also applied because of high potential endogeneity between innovation output (lispe) and productivity (Ivape) according to the empirical literature. Moreover, this is in line with Therrien 
and Hanel (2009) who found, for Canadian data, evidence of endogeneity between those two variables. Therefore, lispe is also instrumented and Ivape is estimated by 2SLS instrumental variables.

\subsubsection{Findings}

The results for the full sample are presented in two tables. Table 8.6-2 reports results from the four equations forming the two Heckman selection models of the first stage. Although the equations were estimated on several different sets of variables, we report only results from our preferred model. Table 8.6-3 reports results from the second and the third stages of the model. In this case, results from our preferred model are presented along with results from alternative specifications for sensibility analysis and robustness check. Furthermore, in order to verify in the case of sponsored firms, the evidence pointed out in the literature, concerning empirical differences in the performance of firms of different size (You (1995), Lee and Newton (2001)) as well as that of firms of different technological level (Balcerowicz, Peczkowski and Wziatek-Kubiak (2009), Kirner, Kinkel and Jaeger (2009)), additional estimations were conducted using different sub-samples. The results are reported in Table 8.B-2. More precisely, we used the following four subsamples: medium-low and low technology, high and medium high technology, SME's and large firms. For this last case however, because of an insufficient number of observations, we could not conduct the analysis for the entire model, which is very demanding in this regard. Hence, estimation results in Table 8.B-2 exclude those relating to large firms. Also, for the sake of brevity, only the results for the second and third stages are reported.

\section{Selection equation for government support}

The selection equation for government support is estimated on the total sample which includes recipients and non-recipients firms (2,166 observations) in order to determine which firms are more likely to use public sponsored programs. It uses a probit model to estimate the probability of using programs sponsored by the federal or the provincial governments. The results for this equation are shown in Column 1 of Table 8.6-2 and the coefficients reported are marginal effects. As can be seen, several factors affect this probability. Firm size (lemp) effect is significant, suggesting that the probability to use public sponsored programs increases with the size of the firm. The share of personnel working in R\&D activities (engineers, scientists) which is a good indicator of firms' involvement in innovation activities also has a significant positive coefficient, thus adding to the evidence that the probability to use public sponsored programs is affected by the share of R\&D personnel in the firm (Baghana (2010)). The highly significant positive effects on export_ot (1.108) and on export_us (0.461) indicate that exporting firms (to both 
the US and the rest of the world) have a higher probability of being sponsored by public programs than firms that do not export. Czarnitzki and Licht (2006) found similar results for firms in Eastern and Western Germany. The last factor entering into the equation with significant positive effect is the indicator variable for contracting out R\&D services. This result is in line with the fact that most R\&D programs from the federal and the provincial governments recognize $R \& D$ contracted out and entitle firms that do so with the same incentive than firms doing R\&D themselves. Finally, for market share ( $\left.m k t_{-} s h r\right)$ and the concentration ratio (cr4), we fail to reject the hypothesis that their respective coefficients are null.

\section{Government support equation}

Table 8.6-2, Column 2, reports the results for the intensity of the government support given by the amount of government support per employee. All coefficients, except that of the indicator variable for contracting out R\&D services, are statistically significant at the $1 \%$ level. Estimation results indicate that the intensity of the government support varies positively with capital intensity, the share of R\&D personnel, the share of sales exported outside Canada and the concentration ratio, and negatively with firm size and market share. Results also indicate that contracting out R\&D services does not affect the magnitude of government support. This result is important since it provides econometric evidence about theoretical determinants of the intensity of government support, using actual amounts of government support received by firms instead of binary variables used in most studies. While the meaning of the positive coefficients reported is straightforward, that of the negative ones may be intriguing. In the case of size, that is proxied by the log of employment, the explanation lies in the fact that most of the public support provided to firms in the estimation sample comes from the main fiscal measure for R\&D support of the Government of Québec, which subsidizes mainly human capital, namely the refundable tax credit for salaries and wages of researchers. For larger firms, there is a ceiling from where the magnitude of the government support decreases, hence explaining the negative coefficient. In the same vein, since firms with the largest market share tend to be large firms, it is straightforward that the magnitude of the government support varies negatively with market share as well.

\section{Innovation selection equation}

The selection equation of innovation identifies factors influencing firms' decisions to be innovative. The estimated regression marginal effects for this equation are presented in Column 3 of Table 8.6-2. The magnitude of government support (represented by the predicted log of government support per employee (Igovsuppe) (computed in the first model of Heckman) is, without surprise, positive and highly significant: an increase of $1 \%$ in government support is associated with a $0.32 \%$ 
higher probability of being innovative. A positive effect is also found by Halpern and Muraközy (2010) using Hungarian CIS data on a CDM model that include, among explanatory variables, a binary for government support in all the equations. They report a lower marginal effect (0.104), but highly significant.

Results in Column 3 also show the other factors influencing firms' decisions to be innovative.Factors influencing positively this probability are the size of the firm, the factors affecting firm's success (developing custom-designed products for clients, developing niche, specialized markets, export markets or domestic markets) and using strategic methods for intellectual property protection (patents, trademarks, copyrights, confidentiality agreements or secrecy, complexity of design or lead-time advantage on competitors). Factors affecting negatively this probability are the firm' share of sales exported outside Canada and the concentration ratio.

\section{Innovation input equation}

The innovation input equation seeks to pinpoint factors influencing the innovation process. Innovation input may be ICT, R\&D expenditure, training or other intangible assets. In this study, innovation input is measured by the firm's total expenditures devoted to innovation activities per employee. The coefficients reported (Table 8.6-2, Column 4) for coop and rd_out are marginal effects, but for lemp, mkt_shr, export_ot, export_us, cr4 and lgovsuppe, the reported coefficients are direct effects since they also appear in the innovation selection equation ${ }^{48}$.

As can be seen, the estimated elasticity of innovation expenditures with respect to government support is 0.989 . This means that a $1 \%$ increase in government support is associated with an increase in innovation expenditures of $0.99 \%$, that is, almost $1 \%$. The estimated effect for $r d$ out is not significant and the estimated effect for coop is significant and positive, which means that firms that cooperate invest more in innovation expenditure than those that don't. The magnitude of the estimated marginal effect for this variable, $23 \%$, is higher than the one reported by Therrien and Hanel (2009) on an unrestricted sample of Canadian firms (14\%). Firm size is statistically significant at the $1 \%$ level, and is positively correlated to innovation expenditures per employee. Results also show a positive relationship between market share and innovation expenditures and a negative relationship between the concentration ratio and innovation expenditures. Contrary

\footnotetext{
${ }^{48}$ Greene (2003), p. 783, emphasises about selection models that it is quite possible that the magnitude, sign, and statistical significance of the marginal effect might all be different from those of the estimated coefficients. Indeed, in the estimation of selection models such as Heckman models, the marginal effect of the independent variables on the dependant variable of the outcome equation (i.e. Igvosuppe and Irtotpe in the present case) in the observed sample consists of two components. First, there is the direct effect on the mean of the dependant variable, which corresponds to the reported coefficients in the table above. Second, there is an indirect effect if the independent variable also appears in the selection equation (recip and innov in the present case).
} 
to Therrien and Hanel (2009), we can't find any significant effect for the share of sales exported outside Canada.

\section{Innovation output equation}

The innovation output equation seeks to answer the question of knowing whetherspending on innovation input increases sales from product innovations (lispe). Table 8.6-3, Column 5, shows the results for the innovation output equation estimated with the predicted value of innovation expenditures per employee (Irtope), in accordance with the results of the endogeneity tests presented in the preceding section. Column 6 shows the results for the same equation but with a simple OLS estimation. As can be seen in Column 5 , the reported coefficient of 0.833 in the predicted value of Irtotpe is highly significant, leading to say that the elasticity of product innovation sales with respect to innovation expenditures is 0.833. The reported elasticity of 0.281 in the equation with the OLS estimation, conducted for sensitivity analysis, underestimates the impact of innovation expenditures but remains positive and highly significant, thus confirming our result. The reported elasticity of 0.833 is more than double of that reported by Therrien and Hanel (2009) on an unrestricted sample of Canadian manufacturing firms (0.33). Since our sample is restricted to firms receiving government support and taking into account that our model allows controlling for the magnitude of government support through the amounts actually received by firms, we can attribute the higher elasticity found to the effect of public support. The total effect of government support on innovation sales may be calculated by multiplying this estimated elasticity by the 0.989 elasticity of innovation expenditures with respect to government support, which gives a total effect of government support on innovation sales of 0.824 . In other words, a $1 \%$ increase in government support yields a $0.82 \%$ increase in innovation sales.

Concerning the variable process, the dummy indicating whether a firm introduced a new or significantly improved production process, the reported coefficient of 0.182 is not significant. This result is confirmed by the estimation result of the OLS (column 6). Other studies reporting similar results, but based on unrestricted sample, are Therrien and Hanel (2009) for large Canadians firms only, and Halpern and Muraközy (2010). Hence, we can conclude that there is no evidence, in the case of innovative manufacturing firms receiving support from the Government of Québec, that the introduction of a production process innovation positively affects product innovation sales. In other words, we cannot conclude that recipient firms that introduce both product and process innovations get greater returns than those introducing only product innovation.

Other factors that may potentially affect product innovation sales are cr4, the concentration ratio and dgtf, the distance to the global technology frontier. For the concentration ratio, estimation results show a negative but non significant 
coefficient. This provides an indication that recipient firms in more concentrated industries spend more on innovation and earn more sales from product innovation than the other firms. Concerning the global technology frontier, the estimation yields the expected negative coefficient, but the latter is very low $(-0.001)$ and not significant statistically, which is somewhat surprising. This could be due to a measurement issue given the fact that the variable dgtf is constructed from data sourced from several countries.

Finally, the other factors likely to influence product innovation sales are the sources of information for innovation proxied in the model by three dummies, s_intra, s_market and s_pub which are significant in the first case and nonsignificant in the two others. Thus, in the case of recipient firms in Québec, we cannot conclude about the importance of the sources information from market partners such as clients and suppliers (s_market) and from public research institutions (s_public) though Landry and Amara (2003) show positive effects of the these three sources of information on innovation with Canadian data. The evidence seems however mix since Therrien and Hanel (2009) find positive effects only for s_market.

The results for sub-samples in Table 8.B-2 show that, the introduction of improved production process affects positively product innovation sales in recipients SME's (Column 5), but the reported coefficient is near rejection. The coefficients of process in any technological level (Columns 6 and 7) are strongly rejected, thus confirming the result found in the full sample. Internal sources of information on innovation (s_intra) is important for the groups of SME's and of medium-low and low technology firms, but is not the case for high and mediumhigh technology firms. As regards to the elasticity of product innovation sales with respect to innovation expenditures, the results in all sub-samples confirm the full sample result and indicate not surprisingly that the elasticity for the group of high and medium-high technology is higher than that for the group of medium-low and low technology.

\section{Productivity equation}

The productivity equation estimates the link between product innovation sales and labour productivity which, in this study, is given by the log of value added per employee. Estimation results of our preferred model estimated by 2SLS instrumental variables are reported in Column 7 of Table 8.6-3. In addition, we experimented with several specifications of the same model for robustness check and report three other specifications. Column 8 includes two more variables to the equation, respectively human capital ( $h c$ ) as in OECD (2009) and physical capital (lgipe). Column 9 reports the OLS estimation results and Column 10, the estimation results with 2 SLS instrumental variables, using a latent predicted value for innovation output (lispe, the log of product innovation sales per employee) 
computed in the previous stage. As can be seen in Column 7, the coefficient on the instrumented variable lispe, 0.372 , is positive and significant at the $1 \%$ level. Hence, a $1 \%$ increase in firms' product innovation sales per employee is associated with a labour productivity increase of $0.37 \%$. This elasticity is in the same range than those reported for Canadian data in OECD (2009) (0.43) and by Therrien and Hanel (2009) in the case of large firms $(0.35)^{49}$. As already mentioned in Section 8.2, several CDM studies with different specifications also report results with positive and significant elasticity. These include Griffith et al. (2006), Hall et al. (2009), Johansson and Lööf (2009), Berger (2009), Halpern and Muraközy (2010), etc. Comparing the elasticity of our preferred model in Column 7 to that of Column 8 shows that, in general, including human and physical capital does not change significantly the results presented above. More specifically, the elasticity of the instrumented variable lispe decreases to 0.29 but remains positive and strongly significant. Human capital is negative and significant while physical capital is positive and significant, thus indicating that capital-intensive firms earn more innovation sales and have higher productivity than others. Concerning the result for the OLS estimation (Column 9), the computed elasticity is lower but significant and positive. The total effect of government support on the productivity of subsidized firms may be calculated by multiplying the estimated elasticity of labour productivity with respect to product innovation sales $(0.372)$ by the 0.824 elasticity of innovation sales with respect to government support, which gives 0.307 . Hence, raising government support by $1 \%$ causes a $0.31 \%$ increase in labour productivity. These results suggest that subsidized firms are more productive than non-subsidized ones.

The coefficient for process is negative and non-significant, what seems counterintuitive since normally labour productivity is expected to increase following the implementation of a new or significantly improved production process. One possible explanation for this contradiction broadly discussed in the literature as many studies report similar results, might be inevitably the lag between the implementation of an innovation process and its impact on productivity. Among studies reporting similar results, one can cite for example, Johansson and Lööf (2009), Berger (2009) or Halpern and Muraközy (2010). The coefficient on the global technology frontier is negative and, as in the innovation sales equation, it has a very low magnitude. In the present case however, it is significant at the $5 \%$ level. Thus, when the distance from the technology frontier decreases, firms productivity increase. In other words, successful firms are the ones that invest more on innovation or adopt rapidly new knowledge. The low magnitude of the coefficient of the variable $d g t f$ could certainly be improved in a future research by refining this variable.

\footnotetext{
${ }^{49}$ For full sample, Therrien and Hanel (2009) reports also a positive and significant elasticity of productivity with respect to innovation sales per employee of 0.22 .
} 
Finally, concerning the sub-samples presented in Table 8.B-2, results confirm those of the full sample. In particular, process is not significant and the elasticity of labour productivity with respect to product innovative sales is positive and highly significant in all sub-samples. The elasticity for the group of SME's (0.35) is close to that of the full sample (0.37) and it is more than twice as large for the group of high and medium-high technology (0.46) as for the group of medium-low and low technology firms (0.22).

Table 8.6-2: $\quad$ Results for the Québec extended model, first stage, full sample

\begin{tabular}{|c|c|c|c|c|}
\hline & \multicolumn{4}{|c|}{ First stage } \\
\hline & $\begin{array}{c}\text { Government } \\
\text { support selection } \\
\text { equation }\end{array}$ & $\begin{array}{c}\text { Government } \\
\text { support equation }\end{array}$ & $\begin{array}{l}\text { Innovation } \\
\text { selection } \\
\text { equation }\end{array}$ & $\begin{array}{c}\text { Innovation input } \\
\text { equation }\end{array}$ \\
\hline & 1 & 2 & 3 & 4 \\
\hline Dependant variable & $\begin{array}{c}\text { recip } \\
\text { (Heckman } \\
\text { 1:Probit) } \\
\end{array}$ & $\begin{array}{c}\text { lgovsuppe } \\
\text { (Heckman 1: MLE) }\end{array}$ & $\begin{array}{c}\text { innov } \\
\text { (Heckman 2: } \\
\text { Probit) }\end{array}$ & $\begin{array}{c}\text { Irtotpe } \\
\text { (Heckman 2: } \\
\text { MLE) }\end{array}$ \\
\hline \multicolumn{5}{|l|}{ Regressors } \\
\hline lemp & $0.475^{* *}$ & $-0.556 * * *$ & $0.214^{* * *}$ & $0.426 * * *$ \\
\hline lemp2 & -0.036 & & & \\
\hline cap_int & & $0.248 * * *$ & & \\
\hline rd_pers & $0.022 * * *$ & $0.024 * * *$ & & \\
\hline$m k t \_s h r$ & -0.117 & $-2.156 * * *$ & 0.929 & $2.835^{* *}$ \\
\hline export_ot & $1.108^{* * *}$ & $1.310 * * *$ & $-1.019 * * *$ & -0.099 \\
\hline export_us & $0.461 * * *$ & $0.666 * * *$ & $-0.512 * *$ & 0.117 \\
\hline fac_new & & & $0.183^{*}$ & \\
\hline fac_custom & & & $0.341 * * *$ & \\
\hline fac_dev & & & $0.371 * * *$ & \\
\hline coop & & & & $0.233^{* *}$ \\
\hline rd_out & $0.366^{* * *}$ & 0.159 & & 0.156 \\
\hline$c r 4$ & 0.155 & $0.840 * * *$ & $-0.509 *$ & $-0.597 * *$ \\
\hline m_e & & & 0.131 & \\
\hline methode_inst & & & $0.270 * *$ & \\
\hline methode_priv & & & $0.297 * * *$ & \\
\hline methode_stra & & & $0.572 * * *$ & \\
\hline sic1-sic 9 & included & included & included & included \\
\hline region1-region3 & included & included & included & included \\
\hline lgovsuppe (pred) & & & $0.324^{* * *}$ & $0.989 * * *$ \\
\hline Mill $_{1}$ & & & & $1.357 * * *$ \\
\hline Rho & $0.394 * *$ & & $-0.363^{*}$ & \\
\hline Constant & $-2.408 * * *$ & $5.014 * * *$ & $-3.336 * * *$ & -0.283 \\
\hline Observations & 2166 & 2166 & 764 & 764 \\
\hline
\end{tabular}

Notes: $\quad *$ significant at the $10 \%$ level; ${ }^{* *}$ significant at the $5 \%$ level; ${ }^{* * *}$ significant at the $1 \%$ level. The coefficients reported in Columns 1-4 are marginal effects, except for lemp, rd_pers, mkt_shr, export_ot, export_us, rd_out and cr4 in Column 2, and lemp, mkt_shr, export_ot, export_us, cr4 and Igovsuppe in Column 4. For these variables, the reported coefficients are direct effects since they also appear in the selections equations (resp. in Columns 1 and 3$)^{48 .}$ In the innovation selection equation (innov) and in the innovation input equation (Irtotpe), the predicted value of the intensity of government support (Igovsuppe) is used rather than its actual value. Significance levels in these equations are based on bootstrapped standard errors. 


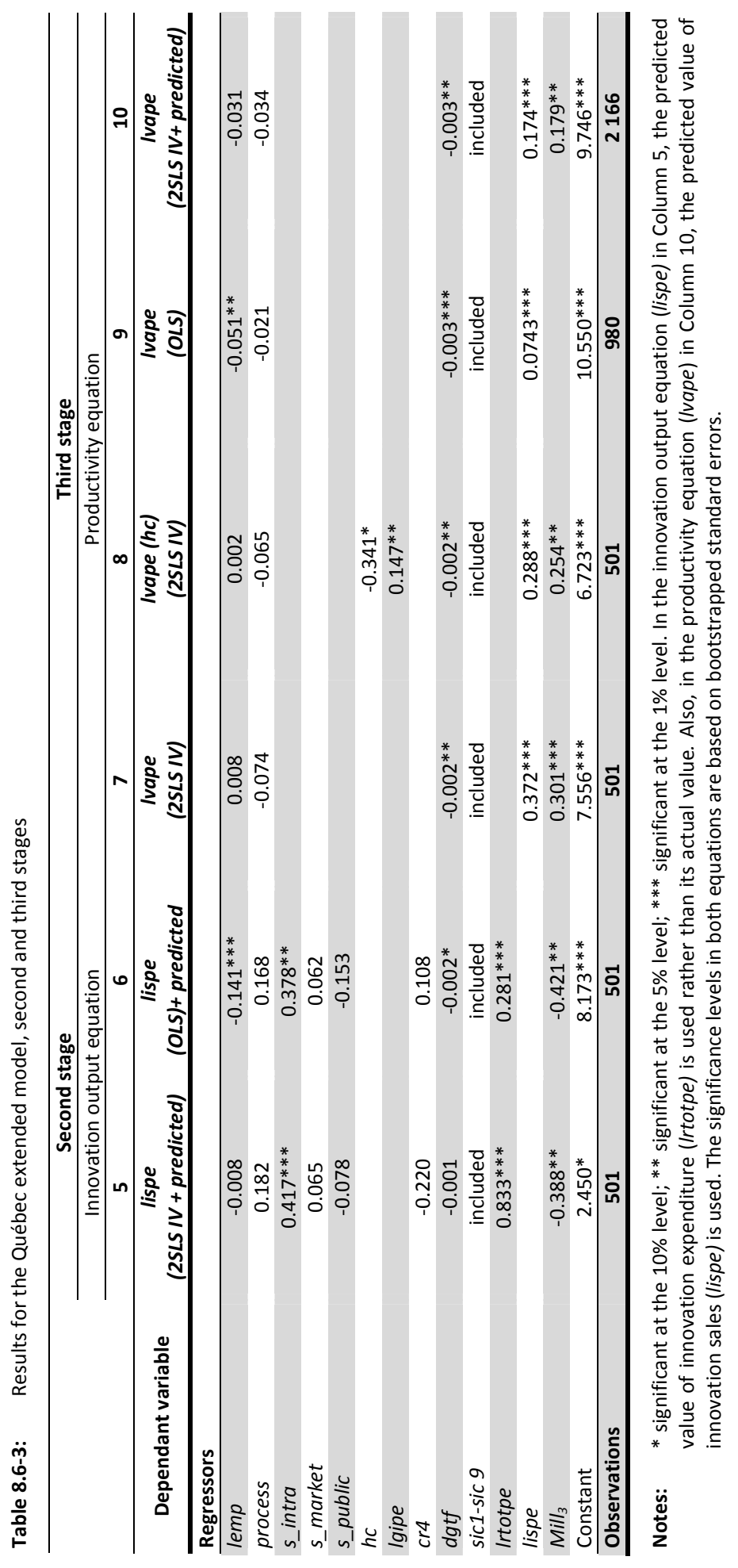




\subsubsection{Comparing Québec to International Econometric Findings (OECD Model)}

The OECD study makes an international comparison of the characteristics of firms and, from these characteristics, tries to identify the link between innovation and productivity. This is a much simpler model than the extended model for Québec presented in the previous section. It doesn't model the effect of government support, nor does it take advantage of all the set of the rich data that comes from innovation surveys. Indeed, in its development stage, several variables have been left apart in order to enable analysis on a larger number of countries. Nevertheless, this model is interesting because of its ability to allow an international comparison on the basis of a single model with harmonized data. We estimated this model with data from Québec to allow empirical evidence on how to compare Québec firms worldwide ${ }^{50}$. The four equations model used allows answering the usual questions of CDM frameworks: Which firms are more likely to be innovative? Which firms invest more on innovation? Does spending in innovation translate into sales from product innovation? What is the impact of product innovation on labour productivity? The following lines present the estimation results from Québec compared to the results obtained by OECD (2009) study.

\section{Which firms are more likely to be innovative?}

Table 8.6-4 shows the results reported in Table 3.1 in Chapter 3 of the OECD (2009) study along with our results for Québec. Coefficients reported are marginal effects from the first equation of the model, which is a probit model estimating the factors influencing the firm's decision to innovate. As can be seen, not surprisingly, results for Québec are quite similar to those of Canada, which themselves are close to those of other countries in the study. There is, however, one exception: the OECD study has found that, unlike other countries, being part of a group is negatively correlated with the probability of innovating in the case of Canada, Korea and Norway. Our results also show a negative marginal effect for this variable, but contrary to Canada these three countries we fail to reject the null that being part of a group is not significant. Despite this, the difference between Québec and Canada seems moderate since in the case of Canada, the marginal effect of -0.105 is significant at the $10 \%$ level only. Thus, being part of a group does not seem to affect the probability to be innovative for Québec firms. For the dummy variable that captures the influence of firms' openness to foreign markets on the decision to innovate, we find a highly significant positive coefficient of 0.332 for Québec. This confirms the result found for the other countries in the OECD study (except for

\footnotetext{
50 Unlike the Québec extended model, the OECD model was estimated on the whole Québec establishments' database which comprises 2908 observations.
} 
Brazil), hence, operating in a foreign market influences positively the probability of firms to innovate. For the size factor, we find that an increase of $1 \%$ in employment raises by more than $11 \%$ the probability of being innovative. This is not much different from the result found in the OECD study for Canada. Finally, as it is the case for Canada, our results for Québec do not include marginal effects for variables that control for the effects of obstacles to innovation (barriers related to knowledge, to markets and to costs). Indeed, for these variables no information is available since the Canadian survey collected such information for innovative firms only.

\section{Which firms invest more on innovation?}

Econometric results on factors that affect firms' investment in innovation are reported in Table 8.6-5 (results from Table 3.2 in Chapter 3 of the OECD (2009) study, except for Québec). Factors influencing firm's investment in innovation are operating in a foreign market, being engaged in co-operation and receiving financial public support. In all cases, the results for Québec support those in the OECD study. Particulary, cooperation and public financial support are strongly correlated with innovation expenditures. We find that firms that cooperate spend almost $31 \%$ more in innovation than firms that do not and those that receive public financial support spend $24 \%$ higher than average. Compared to Canada as a whole, the results of Québec are higher for these two factors for which the results found by the OECD study reported rates of respectively $17 \%$ for co-operation and $18 \%$ for public financial support. In the first case, the difference could be explained by the fact that part of public financial support in Québec is built on encouraging R\&D made through private partnerships or as part of a research consortium. In the second case, this difference could simply be attributed to the fact that on average the magnitude of public support available to firms located in Québec is higher than that available to firms located elsewhere in Canada.

\section{Does spending in innovation translate into sales from product innovation?}

The OECD study does not report the table of estimation results as for the two previous equations, but it states that investing in innovation is associated with an increase in sales from product innovation in all countries, except Switzerland. We find a correlation with sales of $35 \%$, which is comprised in the range of $14-40 \%$ found in the OECD study. Size is significant and negatively correlated to sales from product innovation, but we fail to find in Québec, as in the case of the other countries, strong evidence that firms that engage in collaboration with different partners have significantly more innovative sales. 


\section{What is the link between innovation and productivity?}

As in the previous cases, our results for Québec support the ones found in the OECD study. More specifically, we find that a $1 \%$ increase in product innovation sales per employee is associated with an estimated $0.43 \%$ increase in labour productivity. It is interesting to note that this is about the same rate found for Canada $(0.44 \%)$. Regarding process innovation, like for countries in the OECD study, we find a counterintuitive significant coefficient of -012 . However, this negative result is not surprising since in this case too, the coefficient is not much different to that of Canada. 


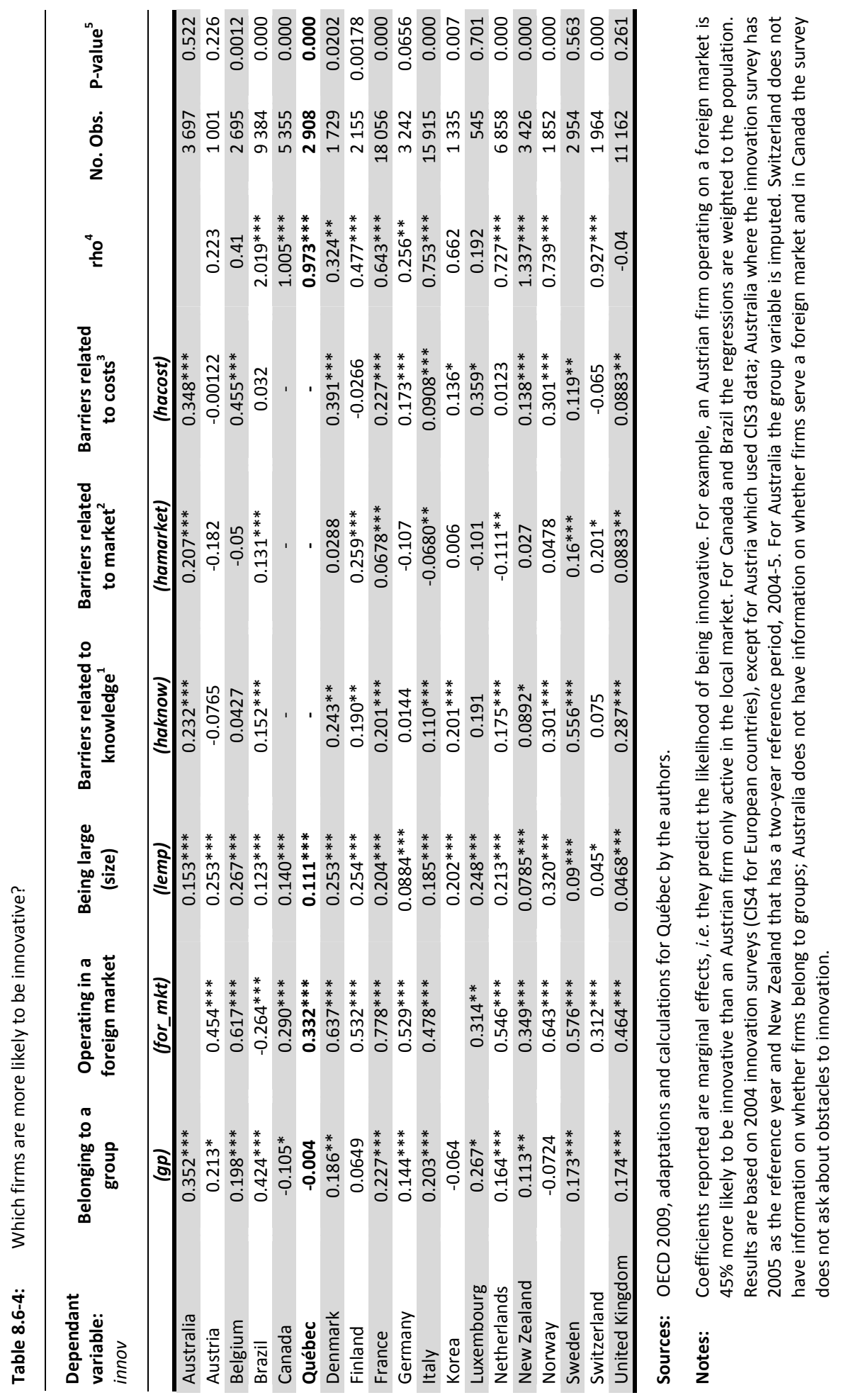




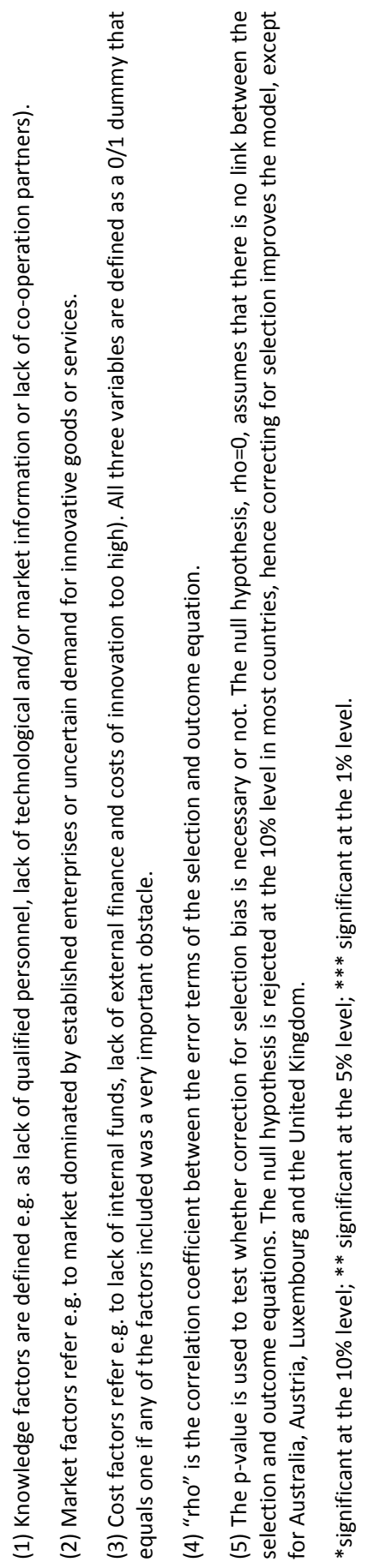


Table 8.6-5: Which firms spend more on innovation?

\begin{tabular}{|c|c|c|c|c|c|}
\hline $\begin{array}{l}\text { Dependant } \\
\text { variable: } \\
\text { Irtope }\end{array}$ & $\begin{array}{l}\text { Belonging to } \\
\text { a group }\end{array}$ & $\begin{array}{l}\text { Operating in a } \\
\text { foreign market }\end{array}$ & $\begin{array}{l}\text { Being engaged } \\
\text { in co-operation }\end{array}$ & $\begin{array}{c}\text { Receiving } \\
\text { financial } \\
\text { public support }\end{array}$ & $\begin{array}{r}\text { No. of } \\
\text { observations }\end{array}$ \\
\hline & (gp) & (for_mkt) & (coop) & (finsup) & \\
\hline Australia & $0.443^{* *}$ & & -0.161 & -0.0334 & 3697 \\
\hline Austria & 0.161 & $0.737 * * *$ & $0.408 * * *$ & $0.746 * * *$ & 1001 \\
\hline Belgium & $0.233^{*}$ & $0.524 * * *$ & -0.0205 & $0.714^{* * *}$ & 2695 \\
\hline Brazil & $0.875^{* * *}$ & $-0.204^{*}$ & $0.384 * * *$ & $0.332 * * *$ & 9384 \\
\hline Canada & $0.145^{*}$ & $0.448 * * *$ & $0.173^{* *}$ & $0.183^{*}$ & 5355 \\
\hline Québec & 0.0691 & $0.306 * * *$ & $0.306 * * *$ & $0.242 * * *$ & 2908 \\
\hline Denmark & $0.477 * * *$ & $0.762 * * *$ & 0.182 & $0.735^{* * *}$ & 1729 \\
\hline Finland & $0.260 * *$ & $0.361^{*}$ & $0.495 * * *$ & $0.460 * * *$ & 2155 \\
\hline France & $0.231 * * *$ & $1.158 * * *$ & $0.427 * * *$ & $0.683^{* * *}$ & 18056 \\
\hline Germany & 0.0538 & $0.610 * * *$ & $0.402 * * *$ & $0.469 * * *$ & 3242 \\
\hline Italy & $0.268 * * *$ & $0.511 * * *$ & $0.310 * * *$ & $0.412 * * *$ & 15915 \\
\hline Korea & -0.167 & & 0.079 & $0.407^{* * *}$ & 1335 \\
\hline Luxembourg & 0.212 & 0.434 & 0.102 & 0.352 & 545 \\
\hline Netherlands & $0.247^{* * *}$ & $0.675^{* * *}$ & $0.389 * * *$ & $0.569 * * *$ & 6858 \\
\hline New Zealand & $0.664 * * *$ & $0.740 * * *$ & $0.225^{* * *}$ & 0.143 & 3426 \\
\hline Norway & -0.0436 & $0.706 * * *$ & $0.354 * * *$ & $0.657 * * *$ & 1852 \\
\hline Sweden & $0.173^{* * *}$ & & $0.576 * * *$ & & 2954 \\
\hline Switzerland & & $-0.717^{* *}$ & $0.370 * *$ & -0.128 & 1964 \\
\hline $\begin{array}{l}\text { United } \\
\text { Kingdom }\end{array}$ & 0.0508 & $0.513^{* * *}$ & $0.377 * * *$ & $0.537 * * *$ & 11162 \\
\hline
\end{tabular}

Notes: Coefficients reported are marginal effects for the co-operation and financial support variables but not for the group and foreign markets variables because the latter enter both the selection (probability to innovate) and the outcome (innovation intensity) equation. When variables enter both the selection and outcome equations their marginal effect can be broken down into two parts: the first is the direct effect on the mean of the dependent variable (which is reported in this table) and the second comes from its effect through its presence in the selection equation.

For Canada and Brazil, the regressions are weighted to the population. Results are based on 2004 innovation surveys (CIS-4 for European countries), except for Austria which used CIS3 data; Australia where the innovation survey has 2005 as the reference year and New Zealand that has a two-year reference period, 2004-5.

Belonging to a group; operating in a foreign market; being engaged in co-operation and receiving financial support are $0 / 1$ dummies.

For Australia the group variable is imputed from responses to the question about whether the enterprise collaborated with other members of their group and is underreported as it omits enterprises that are part of an enterprise group but did not collaborate with other enterprises within the group on innovation projects.

For New Zealand information on innovation expenditure is codified as a categorical variable; to transform it to a continuous variable midpoints of each range are used and multiplied by total reported expenditure.

* significant at the $10 \%$ level; ${ }^{* *}$ significant at the $5 \%$ level; *** significant at the $1 \%$ level.

Source: OECD 2009, adaptations and calculations for Québec by the authors. 
Table 8.6-6: What is the impact of product innovation on labour productivity?

\begin{tabular}{|c|c|c|c|c|c|}
\hline $\begin{array}{l}\text { Dependant } \\
\text { variable: } \\
\text { Ilpe }\end{array}$ & $\begin{array}{l}\text { Belonging } \\
\text { to a group }\end{array}$ & $\begin{array}{c}\text { Being large } \\
\text { (Size) }\end{array}$ & $\begin{array}{c}\text { Having } \\
\text { implemented a } \\
\text { process } \\
\text { innovation } \\
\end{array}$ & $\begin{array}{l}\text { Log innovation } \\
\text { sales per worker } \\
\text { (product } \\
\text { innovation) }\end{array}$ & $\begin{array}{r}\text { No. of } \\
\text { observations }\end{array}$ \\
\hline & (gp) & (lemp) & (process) & (lispe) & \\
\hline Australia & 0.12 & $0.144^{* * *}$ & -0.089 & $0.557^{* * *}$ & 509 \\
\hline Austria & $0.182 * *$ & 0.0111 & 0.0443 & $0.312^{* * *}$ & 359 \\
\hline Belgium & $0.328 * * *$ & -0.003 & $-0.116 * *$ & $0.447 * * *$ & 718 \\
\hline Brazil & $0.183^{* *}$ & $0.140 * * *$ & $-0.211 * * *$ & $0.647 * * *$ & 1954 \\
\hline Canada & $0.250 * * *$ & $0.0772^{* *}$ & $-0.122 * *$ & $0.436 * * *$ & 2273 \\
\hline Québec & $0.264 * * *$ & $0.0515^{*}$ & $-0.120 * * *$ & $0.428 * * *$ & 1292 \\
\hline Denmark & $0.186 * *$ & $0.0732 * * *$ & -0.0405 & $0.345^{* * *}$ & 584 \\
\hline Finland & $0.244^{* * *}$ & $0.0859 * *$ & -0.0677 & $0.314 * * *$ & 698 \\
\hline France & $0.232 * * *$ & $0.0536 * * *$ & $-0.129 * * *$ & $0.474 * * *$ & 2511 \\
\hline Germany & $0.0838 * *$ & $0.0625 * * *$ & $-0.116 * * *$ & $0.500 * * *$ & 1390 \\
\hline Italy & 0.093 & 0.00391 & $-0.192 * *$ & $0.485^{* * *}$ & 747 \\
\hline Korea & $0.171 * * *$ & 0.084 & -0.083 & $0.689 * * *$ & 626 \\
\hline Luxembourg & $0.434 * * *$ & 0.0349 & -0.142 & $0.226^{*}$ & 207 \\
\hline Netherlands & 0.0219 & $0.0902 * * *$ & -0.044 & $0.409 * * *$ & 1374 \\
\hline New Zealand & $0.128 * *$ & $0.0662 * * *$ & $-0.135 * * *$ & $0.682 * * *$ & 993 \\
\hline Norway & $0.256 * * *$ & 0.0407 & -0.0716 & $0.344 * * *$ & 672 \\
\hline Switzerland & & $0.113^{* * *}$ & -0.091 & 0.295 & 394 \\
\hline $\begin{array}{l}\text { United } \\
\text { Kingdom }\end{array}$ & $0.150 * * *$ & $0.058 * * *$ & $-0.121 * * *$ & $0.550 * * *$ & 2989 \\
\hline
\end{tabular}

Notes: For Canada and Brazil the regressions are weighted to the population. Results are based on 2004 innovation surveys (CIS-4 for European countries), except for Austria which used CIS3 data; Australia where the innovation survey has 2005 as the reference year New Zealand that has a two-year reference period, 2004-5. Belonging to a group; and having implemented process innovation are 0/1 dummies. Size is measured as log employment. Industry dummies and inverse Mills ratio are included but not reported.

For Australia the group variable is imputed from responses to the question about whether the enterprise collaborated with other members of their group and is underreported as it omits enterprises that are part of an enterprise group but did not collaborate with other enterprises within the group on innovation projects.

For New Zealand information on innovation sales is codified as a categorical variable; to transform it to a continuous variable midpoints of each range are used and multiplied by total reported expenditure.

For all countries, except Belgium and Korea, significance levels are reported based on bootstrapped standard errors. * ${ }^{*}$ significant at the $10 \%$ level; ${ }^{* *}$ significant at the $5 \%$ level; *** significant at the $1 \%$ level.

Source: OECD 2009, adaptations and calculations for Québec by the authors. 


\subsection{Summary and Conclusions}

Direct and indirect government incentives are mostly the main components in national policies to encourage enterprises to innovate and increase their productivity. The question of evaluating the effectiveness of such public support remains open to debate, although recent studies that have examined the impact of public incentives on innovation and productivity rather show the existence of a positive correlation in most cases. Indeed, some studies show instead that there is no positive link while others show the existence of a crowding-out effect. Critics also raise a methodological weakness due to the fact that the models used do not reflect the heterogeneity of public support received by firms. The objective of this study was to contribute to this debate and, at the same time, to fill the gap in the literature on this issue for Québec.

In this study, two models were estimated: first, a modified and extended CDM model, in which government incentives may increase the profit from innovating, using the portion of the Québec data from the Canadian Survey of innovation 2005. Second, the core OECD model (2009) was estimated using the same data to allow for international comparison.

As regards to the extended model, estimation results are as follows: larger firms, firms with a certain share of $R \& D$ personnel, firms that contract-out $R \& D$ services and firms active in international markets have a higher probability of being sponsored by public programs than others firms. The intensity of the government support varies positively with capital intensity, the share of R\&D personnel, the share of sales exported outside Canada and the concentration ratio, and negatively with firms' size and market share. Factors affecting the firm's decision to be innovative are firms' size, the share of sales exported outside Canada and other factors contributing to the performance of the firm such as developing customdesigned good or services for clients or using intellectual property protection methods. Additionally, an increase of $1 \%$ in government support is associated with a $32 \%$ higher probability of being innovative. Concerning the factors determining innovation input, results show that the probability to invest in innovation expenditures is higher for firms that cooperate than for those that don't. In addition, results show a positive relationship between market share and innovation expenditures. The estimated elasticity of innovation expenditures with respect to government support is 0.989 , which means that a $1 \%$ increase in government support is associated with an increase in innovation expenditures of $0.99 \%$, that is, almost $1 \%$. To the question of knowing whether spending on innovation input increases the sales from product innovation, we find an elasticity of 0.833 . This provides support to the hypothesis that sponsored firms earn more sales from 
product innovation than non-sponsored firms. From our estimation parameters, we find a total effect of government support on innovation sales of 0.824 , meaning that a $1 \%$ increase in government support yields a $0.82 \%$ increase in innovation sales. Surprisingly we could not find evidence, in the case of sponsored innovative firms in the manufacturing sector in Québec, that the introduction of a process innovation positively affects product innovation sales. Also, such evidence could not be found on firm performance (labour productivity). Another variable of interest tested in this study is the distance to the global technology frontier. Results show that when the distance from the technology frontier decreases, firm's productivity increases. Finally, concerning the productivity of innovative firms receiving government incentives, we find that an increase of $1 \%$ in product innovation sales per employee is associated with a productivity increase of $0.37 \%$, which permit to conclude that innovative firms receiving government incentives have higher performance than other firms. Once again, from estimation parameters, we find a total effect of government support on the labour productivity of subsidized firms of 0.307 . Hence, raising government support by $1 \%$ causes a $0.31 \%$ increase in labour productivity. In general, estimation parameters are confirmed by those of the different sub-samples tested. Additionally, new evidence is brought about the differences in the performance of firms of different technology level. In particular, we show that high and medium-high technology sponsored firms earn more product innovation sales than medium-low and low technology ones and that the labour productivity elasticity for the first group is more than twice as large as for the second group.

Concerning the core model of the OECD, estimation results with Québec data were compared to the results obtained for Canada and 17 other OECD and nonOECD countries. In general, the results with this simpler model confirm those obtained for the Québec extended model, with a few exceptions. Additionally, in general, no significant difference in magnitude could be found between the results for Québec using the OECD core model and the results for Canada in the OECD microdata project. Briefly, the main results are the following. As found in the preceding model and in the OECD study, firms of a certain size and active in international markets are the one with a higher probability of being innovative. With regard to the investment effort in innovation, results also confirm those of the OECD study, namely that being engaged in co-operation and receiving financial public support induces higher innovation expenditures. Finally, for labour productivity elasticity, the results yield that a $1 \%$ increase in product innovation sales per employee is on average associated with an estimated $0.43 \%$ increase in labour productivity in Québec, such as was found for Canada in the OECD study.

The results found in this study allow us to draw some lessons. First, these results not only add to the evidence found in several other studies that firms that receive government support perform better than others, but also gives an estimate of the impact of the effect of government support on labour productivity. Second, 
using the results of international comparisons, we have shown that manufacturing firms in Québec that invest in innovation have a performance in respect of labour productivity comparable to that of OECD countries. However, it appears clearly that these firms are not sufficiently numerous to help bridge the productivity gap at the aggregate level of Québec. Consequently, one would think that Québec companies do not invest sufficiently on innovation and this is all the more preoccupying, especially considering that they benefit from fiscal incentives greater than in other Canadian provinces and in most countries of the OECD. However, low productivity could also be linked directly to shortcomings in the innovation process such as inadequate investment in ICT or lack of organization's research efficiency. These questions go beyond the scope of the present study and require further research. For their part, Therrien and Hanel (2011) who obtained results similar to ours on Canadian manufacturing data, suggest that there may be a weak coordination or design of existing government programs involving collaboration and support to business $R \& D$, innovation and cooperation. This is not negligible in the case of Québec, because the incentives offered by the Government of Québec to stimulate cooperation between firms are generally limited to tax credits for R\&D and the statistics show that only a small proportion of firms that conduct R\&D claimed for these tax credits in the last years (Baghana and Mohnen (2009)). Finally, there is also the question of Québec's industrial structure. A study by the HEC Montréal Centre for Productivity and Prosperity (CPP (2010)) concludes that the productivity lag of Québec behind the major industrialized countries is rather a structural problem, in particular the fact that Québec industry is turned towards lowproductivity sectors.

These elements and our results point to the policy recommendation that, in its effort to reduce the productivity gap, the Government of Québec should adopt more active and specific policies of R\&D and innovation support in order to target more specifically the industries or sectors where government intervention is needed. A cross-sector study to identify these last ones could be conducted in a future research. 


\section{Chapter Appendices}

\section{A. Descriptive Statistics of Groups}

Table 8.A-1: Group means comparison between innovators and non-innovators, total sample

\begin{tabular}{|c|c|c|c|c|c|}
\hline Variable & $\begin{array}{c}\text { Mean } \\
\text { innovators } \\
\text { (S.D) }\end{array}$ & $\begin{array}{c}\text { Mean } \\
\text { non-innovators } \\
\text { (S.D) }\end{array}$ & t-stat & $\begin{array}{l}\text { Number of } \\
\text { innovators }\end{array}$ & $\begin{array}{r}\begin{array}{r}\text { Number of } \\
\text { non- } \\
\text { innovators }\end{array} \\
\end{array}$ \\
\hline$g p$ & $\begin{array}{c}0.201 \\
(0.011)\end{array}$ & $\begin{array}{c}0.175 \\
(0.014)\end{array}$ & 1.423 & 1453 & 713 \\
\hline lemp & $\begin{array}{c}3.771 \\
(0.024)\end{array}$ & $\begin{array}{c}3.601 \\
(0.032)\end{array}$ & $4.159 * * *$ & 1453 & 713 \\
\hline lemp2 & $\begin{array}{l}15.048 \\
(0.196)\end{array}$ & $\begin{array}{l}13.711 \\
(0.263)\end{array}$ & $3.984^{* * *}$ & 1453 & 713 \\
\hline cap_int & $\begin{array}{l}11.503 \\
(0.029)\end{array}$ & $\begin{array}{l}11.425 \\
(0.075)\end{array}$ & 0.998 & 659 & 105 \\
\hline rd_pers & $\begin{array}{l}11.440 \\
(0.388)\end{array}$ & $\begin{array}{c}3.958 \\
(0.326)\end{array}$ & $12.498 * * *$ & 1453 & 713 \\
\hline$m k t \_s h r$ & $\begin{array}{c}0.021 \\
(0.001)\end{array}$ & $\begin{array}{c}0.016 \\
(0.002)\end{array}$ & $2.159 * *$ & 1453 & 713 \\
\hline for_mkt & $\begin{array}{c}0.748 \\
(0.011)\end{array}$ & $\begin{array}{c}0.567 \\
(0.019)\end{array}$ & $8.715^{* * *}$ & 1453 & 713 \\
\hline export_ot & $\begin{array}{c}0.049 \\
(0.003)\end{array}$ & $\begin{array}{c}0.028 \\
(0.004)\end{array}$ & $3.689 * * *$ & 1453 & 713 \\
\hline export_us & $\begin{array}{c}0.223 \\
(0.007)\end{array}$ & $\begin{array}{c}0.170 \\
(0.010)\end{array}$ & $4.312^{* * *}$ & 1453 & 713 \\
\hline fac_new & $\begin{array}{c}0.390 \\
(0.013)\end{array}$ & $\begin{array}{c}0.208 \\
(0.015)\end{array}$ & $8.605^{* * *}$ & 1453 & 713 \\
\hline fac_custom & $\begin{array}{c}0.532 \\
(0.013)\end{array}$ & $\begin{array}{c}0.352 \\
(0.018)\end{array}$ & $7.995^{* * *}$ & 1453 & 713 \\
\hline fac_dev & $\begin{array}{c}0.615 \\
(0.013)\end{array}$ & $\begin{array}{c}0.353 \\
(0.018)\end{array}$ & $11.832 * * *$ & 1453 & 713 \\
\hline coop & $\begin{array}{c}0.195 \\
(0.010)\end{array}$ & $\begin{array}{c}0.003 \\
(0.002)\end{array}$ & $12.910 * * *$ & 1453 & 713 \\
\hline rd_out & $\begin{array}{c}0.199 \\
(0.010)\end{array}$ & $\begin{array}{c}0.039 \\
(0.007)\end{array}$ & $10.102 * * *$ & 1453 & 713 \\
\hline process & $\begin{array}{c}0.788 \\
(0.011)\end{array}$ & $\begin{array}{c}0.046 \\
(0.008)\end{array}$ & $45.573 * * *$ & 1453 & 713 \\
\hline s_intra & $\begin{array}{c}0.779 \\
(0.011)\end{array}$ & $\begin{array}{c}0.038 \\
(0.007)\end{array}$ & $45.385^{* * *}$ & 1453 & 713 \\
\hline s_market & $\begin{array}{c}0.618 \\
(0.013)\end{array}$ & $\begin{array}{c}0.038 \\
(0.007)\end{array}$ & $30.727 * * *$ & 1453 & 713 \\
\hline s_public & $\begin{array}{c}0.056 \\
(0.006)\end{array}$ & $\begin{array}{c}0.004 \\
(0.002)\end{array}$ & $5.882 * * *$ & 1453 & 713 \\
\hline$h c$ & $\begin{array}{c}0.082 \\
(0.003)\end{array}$ & $\begin{array}{c}0.055 \\
(0.004)\end{array}$ & $5.764 * * *$ & 1453 & 713 \\
\hline lgipe & $\begin{array}{l}11.649 \\
(0.023)\end{array}$ & $\begin{array}{l}11.566 \\
(0.059)\end{array}$ & 1.440 & 879 & 174 \\
\hline $\mathrm{cr} 4$ & $\begin{array}{c}0.350 \\
(0.005)\end{array}$ & $\begin{array}{c}0.310 \\
(0.007)\end{array}$ & $4.461^{* * *}$ & 1453 & 713 \\
\hline dgtf & $\begin{array}{l}49.917 \\
(0.896)\end{array}$ & $\begin{array}{l}51.375 \\
(1.366)\end{array}$ & -0.912 & 1453 & 711 \\
\hline
\end{tabular}


Chapter 8

\begin{tabular}{|c|c|c|c|c|c|}
\hline Variable & $\begin{array}{c}\text { Mean } \\
\text { innovators } \\
\text { (S.D) }\end{array}$ & $\begin{array}{c}\text { Mean } \\
\text { non-innovators } \\
\text { (S.D) }\end{array}$ & t-stat & $\begin{array}{l}\text { Number of } \\
\text { innovators }\end{array}$ & $\begin{array}{r}\begin{array}{r}\text { Number of } \\
\text { non- }\end{array} \\
\text { innovators }\end{array}$ \\
\hline$\overline{m \_e}$ & $\begin{array}{c}0.688 \\
(0.012)\end{array}$ & $\begin{array}{c}0.433 \\
(0.019)\end{array}$ & $11.714^{* * *}$ & 1453 & 713 \\
\hline methode_inst & $\begin{array}{c}0.422 \\
(0.013)\end{array}$ & $\begin{array}{c}0.297 \\
(0.017)\end{array}$ & $5.648 * * *$ & 1453 & 713 \\
\hline methode_priv & $\begin{array}{c}0.539 \\
(0.013)\end{array}$ & $\begin{array}{c}0.292 \\
(0.017)\end{array}$ & $11.152^{* * *}$ & 1453 & 713 \\
\hline methode_stra & $\begin{array}{c}0.604 \\
(0.013)\end{array}$ & $\begin{array}{c}0.269 \\
(0.017)\end{array}$ & $15.431 * * *$ & 1453 & 713 \\
\hline finsup & $\begin{array}{c}0.719 \\
(0.012)\end{array}$ & $\begin{array}{c}0.222 \\
(0.016)\end{array}$ & $24.761^{* * *}$ & 1453 & 713 \\
\hline recip & $\begin{array}{c}0.454 \\
(0.013)\end{array}$ & $\begin{array}{c}0.149 \\
(0.013)\end{array}$ & $14.616^{* * *}$ & 1453 & 713 \\
\hline Igovsuppe & $\begin{array}{c}7.196 \\
(0.051)\end{array}$ & $\begin{array}{c}6.497 \\
(0.155)\end{array}$ & $4.961 * * *$ & 659 & 106 \\
\hline lispe & $\begin{array}{l}10.006 \\
(0.037)\end{array}$ & $\begin{array}{l}10.311 \\
(0.227)\end{array}$ & -1.246 & 958 & 22 \\
\hline Ivape & $\begin{array}{l}11.161 \\
(0.014)\end{array}$ & $\begin{array}{l}11.091 \\
(0.021)\end{array}$ & $2.871 * * *$ & 1453 & 713 \\
\hline Ilppe & $\begin{array}{l}12.026 \\
(0.014)\end{array}$ & $\begin{array}{l}12.011 \\
(0.023)\end{array}$ & 0.597 & 1453 & 713 \\
\hline
\end{tabular}

Note: $\quad * * *$ indicates significance at $1 \%, * *$ at $5 \%$ and $*$ at $10 \%$ level. 


\section{B. Econometric Findings from the Extended Model for Québec (Sub-Samples)}

Table 8.B-2: $\quad$ Findings from SME's and technology industries samples

\begin{tabular}{|c|c|c|c|c|c|c|}
\hline & \multicolumn{3}{|c|}{ Second stage } & \multicolumn{3}{|c|}{ Third stage } \\
\hline & \multicolumn{3}{|c|}{ Innovation output equation } & \multicolumn{3}{|c|}{ Productivity equation } \\
\hline & 5 & 6 & 7 & 8 & 9 & 10 \\
\hline & SME'S & $\begin{array}{l}\text { Medium- } \\
\text { low + Low }\end{array}$ & $\begin{array}{c}\text { High + } \\
\text { Medium- } \\
\text { high }\end{array}$ & SME's & $\begin{array}{l}\text { Medium- } \\
\text { low + Low }\end{array}$ & $\begin{array}{c}\text { High + } \\
\text { Medium- } \\
\text { high }\end{array}$ \\
\hline $\begin{array}{l}\text { Dependant } \\
\text { variable }\end{array}$ & $\begin{array}{c}\text { lispe } \\
\text { (2SLS IV + } \\
\text { predicted) } \\
\end{array}$ & $\begin{array}{c}\text { lispe } \\
\text { (2SLS IV + } \\
\text { predicted) }\end{array}$ & $\begin{array}{c}\text { lispe } \\
\text { (2SLS IV + } \\
\text { predicted) }\end{array}$ & $\begin{array}{c}\text { Ivape } \\
\text { (2SLS IV) }\end{array}$ & $\begin{array}{c}\text { Ivape } \\
\text { (2SLS IV) }\end{array}$ & $\begin{array}{c}\text { Ivape } \\
\text { (2SLS IV) }\end{array}$ \\
\hline \multicolumn{7}{|l|}{ Regressors } \\
\hline lemp & -0.106 & -0.011 & -0.072 & -0.017 & -0.027 & 0.016 \\
\hline process & $0.206^{*}$ & 0.262 & 0.122 & -0.080 & -0.102 & 0.021 \\
\hline s_intra & $0.406 * *$ & $0.427^{*}$ & 0.403 & & & \\
\hline s_market & 0.151 & 0.194 & -0.045 & & & \\
\hline s_public & -0.003 & -0.065 & -0.089 & & & \\
\hline $\mathrm{cr} 4$ & -0.003 & -0.065 & -0.089 & & & \\
\hline$d g t f$ & -0.002 & -0.001 & -0.002 & $-0.002^{* *}$ & $-0.005^{* * *}$ & -0.001 \\
\hline sic1-sic 9 & included & included & included & included & included & included \\
\hline Irtotpe (pred) & $0.595^{* *}$ & $0.713^{* *}$ & $0.883^{* *}$ & & & \\
\hline lispe & & & & $0.349 * * *$ & $0.215^{* * *}$ & $0.458 * *$ \\
\hline $\mathrm{Mill}_{3}$ & $-0.369 * *$ & -0.352 & -0.411 & $0.310 * * *$ & 0.041 & $0.382^{*}$ \\
\hline Constant & $4.675^{*}$ & 3.447 & 2.122 & $7.877 * * *$ & $9.364 * * *$ & $6.985^{* * *}$ \\
\hline Observations & 471 & 323 & 178 & 471 & 323 & 178 \\
\hline
\end{tabular}

Notes: $\quad *$ significant at the $10 \%$ level; ${ }^{* *}$ significant at the $5 \%$ level; ${ }^{* *}$ significant at the $1 \%$ level. In the innovation output equation lispe (Columns 5, 6 and 7), the predicted value of innovation expenditure (Irtotpe) is used rather than its actual value. 


\section{C. Main Economic Regions of Québec}

Table 8.C-3: Main economic regions of Québec

\begin{tabular}{ll}
\hline National Capital region & \\
\hline Region 03 & Capitale-Nationale \\
\hline Montreal and Laval region & \\
\hline Region 06 & Montreal \\
Region 13 & Laval \\
\hline Central region & \\
\hline Region 05 & Estrie \\
\hline Region 07 & Outaouais \\
\hline Region 12 & Chaudière-Appalaches \\
\hline Region 14 & Lanaudière \\
\hline Region 15 & Laurentides \\
\hline Region 16 & Montérégie \\
\hline Region 17 & Centre-du-Québec \\
\hline Resources regions & \\
\hline Region 01 & Bas-Saint-Laurent \\
\hline Region 02 & Saguenay-Lac-Saint-Jean \\
\hline Region 04 & Mauricie \\
\hline Region 08 & Abitibi-Témiscamingue . \\
\hline Region 09 & Côte-Nord \\
\hline Region 10 & Nord-du-Québec \\
\hline Region 11 & Gaspésie--iles-de-la-Madeleine \\
\hline
\end{tabular}




\section{D. Survey of Innovation 2005 - Questionnaire and Corresponding variables}

\section{General Information}

1. In this questionnaire, we refer both to your "plant" and to your "firm". By "plant", we refer to your local operations (as described on the label on the first page of the questionnaire). By "firm", we refer to all plants and operations in Canada or in other countries that comprise your company.

Are the operations of your plant part of a larger firm?

$G P$

$\begin{array}{lllll}0100 & 1 & \bigcirc & \text { Yes } & \\ & 3 & \bigcirc & \text { No } & \text { Please go to Question 2 }\end{array}$

$\underline{\text { If yes, }}$ please indicate the geographical location(s) of other plants and operations in your firm.

\begin{tabular}{|c|c|c|c|c|}
\hline & & & Yes & No \\
\hline 0101 & $\mathrm{a}$ & $\begin{array}{l}\text { In } \\
\text { province/territory your }\end{array}$ & $1 \bigcirc$ & $3 \bigcirc$ \\
\hline 0102 & $\mathrm{~b}$ & In the rest of Canada & $1 \bigcirc$ & $3 \bigcirc$ \\
\hline 0103 & c & United States & $1 \bigcirc$ & $3 \bigcirc$ \\
\hline 0104 & $d$ & Mexico & $1 \bigcirc$ & $3 \bigcirc$ \\
\hline 0105 & $\mathrm{e}$ & Europe & $1 \bigcirc$ & $3 \bigcirc$ \\
\hline 0106 & $f$ & Asia Pacific & $1 \bigcirc$ & $3 \bigcirc$ \\
\hline 0107 & g & All other countries & $1 \bigcirc$ & $3 \bigcirc$ \\
\hline
\end{tabular}

2. Please estimate (as best you can) the percentage of your full-time employees in your plant in 2004 who had:

0201 a A university degree

0202 b A college/technical institute diploma

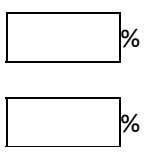

3. Please estimate (as best you can) the percentage of the full-time employees in RD_PERS your plant in $\mathbf{2 0 0 4}$ who were involved in research and development activities.

0301

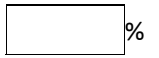

4. Please estimate (as best you can) the percentage of the full-time employees in your plant in $\mathbf{2 0 0 4}$ who were involved in marketing, sales or client services.

0401

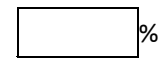


5. Please estimate (as best you can) the percentage of your plant's total revenue that came from the sale of products (goods or services) to clients in the following geographical markets in 2004.

$\begin{array}{lll}0501 & \text { a } & \text { In your province/territory } \\ 0502 & \text { b } & \text { In the rest of Canada } \\ 0503 & \text { c } & \text { United States } \\ 0504 & \text { d } & \text { Mexico } \\ 0505 & \text { e } & \text { Europe } \\ 0506 & f & \text { Asia Pacific } \\ 0507 & \text { g } & \text { All other countries }\end{array}$
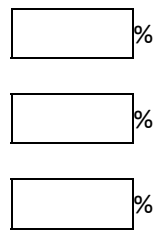

EXPORT_US

EXPORT_OT

EXPORT_OT

EXPORT_OT

EXPORT_OT

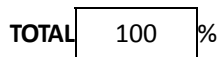

6. Please estimate (as best you can) the percentage of your plant's total revenue in $\underline{\mathbf{2 0 0 4}}$ that came from other plants in your firm?

(If not applicable, indicate 0\%)

0601

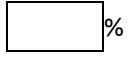

7. Please estimate (as best you can) the percentage of your plant's total revenue in $\underline{\mathbf{2 0 0 4}}$ that came from your most important customer or client (in terms of revenue) who is not a part of your firm.

0701

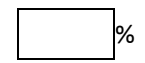

\section{Success factors}

8. Please rate the importance of each of the following factors for the success of your plant during the three years, 2002 to 2004 .

\section{Market and products}

Degree of Importance

\begin{tabular}{|c|c|c|c|c|c|c|c|}
\hline \multirow[b]{2}{*}{0801} & \multirow[b]{2}{*}{ a } & \multirow{2}{*}{$\begin{array}{l}\text { Market and products } \\
\text { Seeking new markets }\end{array}$} & \multirow{2}{*}{$\begin{array}{l}\text { High } \\
5 \bigcirc\end{array}$} & \multirow{2}{*}{$\begin{array}{l}\text { Medium } \\
3 \bigcirc\end{array}$} & \multirow{2}{*}{$\begin{array}{l}\text { Low } \\
1 \bigcirc\end{array}$} & \multicolumn{2}{|c|}{$\begin{array}{l}\text { Not } \\
\text { relevant }\end{array}$} \\
\hline & & & & & & $0 \bigcirc$ & FAC_NEW \\
\hline 0802 & $b$ & Satisfying existing clients & $5 \bigcirc$ & $3 \bigcirc$ & 10 & $0 \bigcirc$ & FAC_EXIST \\
\hline 0803 & c & $\begin{array}{l}\text { Developing niche or specialized } \\
\text { markets }\end{array}$ & $5 \bigcirc$ & $3 \bigcirc$ & $1 \bigcirc$ & $0 \bigcirc$ & $F A C \_D E V$ \\
\hline 0804 & d & Developing export markets & $5 \bigcirc$ & $3 \bigcirc$ & $1 \bigcirc$ & $0 \bigcirc$ & $F A C \_D E V$ \\
\hline 0805 & e & Developing domestic markets & $5 \bigcirc$ & $3 \bigcirc$ & 10 & $0 \bigcirc$ & $F A C \_D E V$ \\
\hline
\end{tabular}




\begin{tabular}{|c|c|c|c|c|c|c|}
\hline \multirow{2}{*}{\multicolumn{2}{|c|}{$0806 f$}} & $\begin{array}{l}\text { Developing custom-designed } \\
\text { products (goods or services) for } \\
\text { clients }\end{array}$ & $5 \bigcirc$ & $3 \bigcirc$ & $1 \bigcirc$ & $0 \bigcirc$ \\
\hline & & Other & & & & \\
\hline 0807 & g & $\begin{array}{l}\text { Active involvement in developing } \\
\text { new industry-wide standards }\end{array}$ & $5 \bigcirc$ & $3 \bigcirc$ & 10 & $0 \bigcirc$ \\
\hline 0808 & $\mathrm{~h}$ & $\begin{array}{l}\text { Ability to comply with } \\
\text { environmental standards and } \\
\text { regulations }\end{array}$ & 50 & 30 & 10 & $0 \bigcirc$ \\
\hline 0809 & $\mathrm{i}$ & $\begin{array}{l}\text { Implementing new information } \\
\text { and communications } \\
\text { technologies }\end{array}$ & $5 \bigcirc$ & $3 \bigcirc$ & $1 \bigcirc$ & 0 \\
\hline
\end{tabular}

\section{Product (good or service) innovation}

9. A PRODUCT INNOVATION is the market introduction of a new good or service or a significantly improved good or service. The innovation (new or improved) must be new to your plant. Exclude the simple resale of new goods purchased from other plants and changes of a solely aesthetic nature.

During the three years, $\mathbf{2 0 0 2}$ to $\mathbf{2 0 0 4}$, did your plant introduce:

$\begin{array}{llllll}0901 & \text { a } & \text { New or significantly improved goods } & \text { Yes } & \text { No } \\ 0902 & \text { b } & \text { New or significantly improved services } & 1 \bigcirc & 3 \bigcirc & \text { INPDGD }\end{array}$

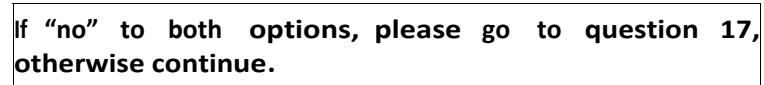

10. During the three years, $\mathbf{2 0 0 2}$ to 2004, how many new or significantly improved products (goods or services) did your plant introduce onto the market?

\section{Number of product innovations}

$\begin{array}{lll}1001 \text { a } & \begin{array}{l}\text { Number of new or significantly } \\ \text { improved goods }\end{array} \\ 1002 \text { b } & \begin{array}{l}\text { Number of new or significantly } \\ \text { improved services }\end{array}\end{array}$

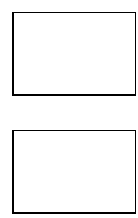

11. Who developed these product (good or service) innovations during the three INPDTW years, 2002 to $\mathbf{2 0 0 4}$ ?

(Select the most appropriate option only)

$\begin{array}{llll}1101 & 1 & \bigcirc & \text { Mainly your plant or your firm } \\ 2 & \bigcirc & \text { Your plant together with other firms or organizations } \\ 3 & \bigcirc & \text { Mainly other firms or organizations }\end{array}$


12. Did your plant introduce ANY new or significantly improved products (goods or NEWMKT services) onto your market before your competitors during the three years, $\underline{\mathbf{2 0 0 2}}$ to 2004?

$\begin{array}{llll}1201 & 1 & \bigcirc & \text { Yes } \\ & 3 & \bigcirc & \text { No Please go to question } 13\end{array}$

If yes, please estimate (as best you can) the percentage of your revenue in $\underline{2004}$ from these first-to-the-market product (good or service) innovations introduced during the three years, 2002 to 2004.

1201

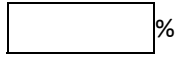

13. Did your plant introduce ANY new or significantly improved products (goods or NEWFRM services) onto your market that were already available from your competitors, during the three years, 2002 to 2004 ?

$\begin{array}{llll}1301 & 1 & \bigcirc & \text { Yes } \\ & 3 & \bigcirc & \text { No Please go to question } 13\end{array}$

If yes, please estimate (as best you can) the percentage of your revenue in 2004 from these already-on-the-market product (good or service) innovations that were introduced during the period, 2002 to 2004.

1302

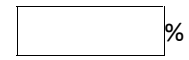

14. On average, how long does it take for your plant to develop a new or significantly improved product (good or service)?

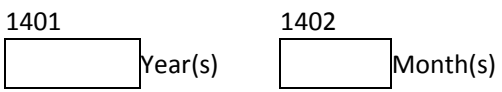

15. During the three years, $\mathbf{2 0 0 2}$ to $\mathbf{2 0 0 4}$, were ANY of your new or significantly improved products (goods or services) introduced by your plant:

\begin{tabular}{llllll}
1501 & a & A first in your province/territory & Yes & No & $\begin{array}{c}\text { Do not } \\
\text { know }\end{array}$ \\
1502 & b & A first in Canada & $1 \bigcirc$ & $3 \bigcirc$ & $0 \bigcirc$ \\
1503 & c & A first in North America? & $1 \bigcirc$ & $3 \bigcirc$ & $0 \bigcirc$ \\
1504 & d & A world first? & $1 \bigcirc$ & $3 \bigcirc$ & $0 \bigcirc$ \\
\hline
\end{tabular}


16. During the three years, $\mathbf{2 0 0 2}$ to $\mathbf{2 0 0 4}$, were ANY of your plant's new or significantly improved products (goods or services) sold to the following natural resource industries?

$\begin{array}{lllcc}1601 & \text { a } & \text { Forestry and logging } & \text { Yes } & \text { No } \\ 1602 & \text { b } & \text { Wood products manufacturers } & 1 \bigcirc & 3 \bigcirc \\ 1603 & \text { c } & \begin{array}{l}\text { Pulp and paper products } \\ \text { manufacturers }\end{array} & 1 \bigcirc & 3 \bigcirc \\ & & & 3 \bigcirc\end{array}$

\section{Process innovation}

17. A PROCESS INNOVATION is the implementation of a new or significantly improved production process, distribution method, or support activity for your goods or services. The innovation (new or improved) must be new to your plant.

During the three years, 2002 to 2004 , did your plant introduce:

PROCESS

$\begin{array}{llllll}1701 \text { a } & \text { Yes } & \text { No } & \\ 1702 \text { b or significantly improved methods of manufacturing } & 1 & 3 \bigcirc & \begin{array}{l}\text { New or significantly improved logistics, delivery or } 1 \bigcirc \\ \text { distribution methods for your inputs, goods or services }\end{array} & 3 \bigcirc & \text { INPSLG } \\ 1703 \text { c } \quad \text { New or significantly improved supporting activities for } 1 \bigcirc & 3 \bigcirc & \text { INPSSU }\end{array}$ your processes, such as maintenance systems or operations for purchasing, accounting, or computing

If "no" to all options, please go to question 20 , otherwise continue.

18. Who developed these process innovations during the three years, 2002-2004?

INPCSW

(Select the most appropriate option only)

$18011 \bigcirc$ Mainly your plant or your firm

$2 \bigcirc$ Your plant together with other firms or organizations

$3 \bigcirc$ Mainly other firms or organizations

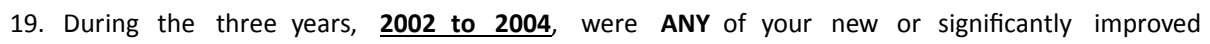
processes:

\begin{tabular}{llllll} 
& & & Yes & No & $\begin{array}{c}\text { Do not } \\
\text { know }\end{array}$ \\
1901 & a & A first in your province/territory & $1 \bigcirc$ & $3 \bigcirc$ & $0 \bigcirc$ \\
1902 & b & A first in Canada & $1 \bigcirc$ & $3 \bigcirc$ & $0 \bigcirc$ \\
1903 & c & A first in North America? & $1 \bigcirc$ & $3 \bigcirc$ & $0 \bigcirc$ \\
1904 & d & A world first? & $1 \bigcirc$ & $3 \bigcirc$ & $0 \bigcirc$ \\
\hline
\end{tabular}




\section{Ongoing or abandoned product and/or process innovations}

20. Did your plant have ANY activities to develop product (good or service) or process innovations that were still ongoing at the end of 2004?

$2001 \quad 1 \quad$ Yes

21. Did your plant have ANY activities to develop product (good or service) or/NONAB process innovations that were abandoned during the three years, $\underline{\mathbf{2 0 0 2}}$ to 2004?

$\begin{array}{llll}2101 & 1 & \bigcirc & \text { Yes } \\ & 3 & \bigcirc & \text { No }\end{array}$

Did your plant introduce ANY new or significantly improved innovations products or processes) during the three years, 2002-2004? (I.e. Did you answer "yes" to any item in question 9 or question 17?)

22. Why did your plant not develop or introduce any new or significantly improved product (goods or services) or processes during the three years, 2002 to 2004?

$\begin{array}{lllll} & & & \text { Yes } & \text { No } \\ 2201 & \text { a } & \text { Innovations were carried out prior to } 2002 \text { to } 2004 & 1 \bigcirc & 3 \bigcirc \\ 2202 & \text { b } & \text { Market doesn't require new products (goods or services) } & 1 \bigcirc & 3 \bigcirc \\ 2203 & \text { c } & \text { Lack of funds to carry out innovation projects } & 1 \bigcirc & 3 \bigcirc \\ 2204 & \text { d } & \text { Lack of trained staff to carry out innovation projects } & 1 \bigcirc & 3 \bigcirc \\ 2205 & \text { e } & \begin{array}{l}\text { Other reasons } \\ \text { please specify: }\end{array} & 1 \bigcirc & 3 \bigcirc\end{array}$

\section{Please proceed to Question 31.}

\section{Innovation activities}

23. During the three years, $\mathbf{2 0 0 2}$ to $\mathbf{2 0 0 4}$, did your plant engage in the following innovatiol activities?

\section{Internal R\&D (in plant)}

2301 a Research and development (R\&D) linked to new or significantly improved products (goods or services) or

\section{Yes No} processes carried out in your plant 


\section{R\&D performed by larger firm}

2302 b The same activities as above, but performed on your

behalf by other plants or R\&D laboratories that are

part of the larger firm to which your plant belongs

\section{External R\&D}

2303 c Same activities as above, but performed by other firms or by public or private research organizations and purchased by your plant

\section{Acquisition of machinery, equipment and software}

$2304 \mathrm{~d}$ Acquisition of advanced machinery, equipment or computer hardware or software to produce new or significantly improved products (goods or services) or processes

\section{Acquisition of other external knowledge}

2305 e Purchase or licensing of patents and non-patented inventions, know-how, and other types of knowledge from other firms or organizations

\section{Training}

$2306 \mathrm{f}$ Internal or external training for your personnel specifically for the development and/or introduction of new or significantly improved products (goods or services) or processes

\section{Market introduction of innovations}

Activities for the market introduction of your new or significantly improved products (goods or services):

$\begin{array}{lll}2307 & \text { g } & \text { Market research } \\ 2308 & \text { h } & \text { Launch advertising } \\ 2309 & \text { i } & \text { Market plan } \\ 2310 & \text { j } & \text { Product positioning or profiling } \\ 2311 & \text { k } & \text { Profitability analysis } \\ 2312 & \text { I } & \text { Project feasibility study } \\ 2313 & \text { m } & \text { Consumer acceptance testing } \\ 2314 & \text { n } & \begin{array}{l}\text { Other, } \\ \text { specify: }\end{array}\end{array}$

\section{Post-introduction commercialization}

Activities undertaken to assure the commercial success of your new or significantly improved products (goods or services):

$\begin{array}{cc}\text { Yes } & \text { No } \\ 1 \bigcirc & 3 \bigcirc \\ 1 \bigcirc & 3 \bigcirc \\ 1 \bigcirc & 3 \bigcirc \\ 1 \bigcirc & 3 \bigcirc \\ 1 \bigcirc & 3 \bigcirc\end{array}$


24. Please estimate (as best you can) the percentage of your plant's total expenditures in $\underline{\mathbf{2 0 0 4}}$ that were devoted to $\underline{\mathbf{A L L}}$ innovation activities described in Question 23.

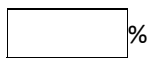

\section{Sources of information and co-operation for innovation activities}

25. During the three years, $\mathbf{2 0 0 2}$ to $\mathbf{2 0 0 4}$, how important to your plant's innovation activities were each of the following information sources? Please identify information sources that provided information for new innovation projects, contributed to the completion of existing innovation projects, or provided information for the commercialization of innovation.

Information sources

Degree of Importance

\begin{tabular}{lll}
\hline High Medium Low & $\begin{array}{l}\text { Not } \\
\text { relevant }\end{array}$
\end{tabular}

Internal sources

S_INTRA

$\begin{aligned} 2501 \text { a } & \text { Research and development } 50 \\ & \text { (R\&D) staff }\end{aligned}$

$\begin{array}{llllll}2502 \text { b Sales and marketing staff } \quad 5 \bigcirc & 3 \bigcirc & 1 & 0\end{array}$

$\begin{array}{lllll}2503 \text { c Production staff } & 5 \bigcirc & 3 \bigcirc & 10 & 0 \bigcirc\end{array}$

2504 d Management staff $\quad 5 \bigcirc \quad 3 \bigcirc \quad 10 \quad 0 \bigcirc$

$\begin{array}{lllll}2505 \text { e } & \text { Other plants or research } \quad 5 \bigcirc & 3 & 0 & 1\end{array}$

and development (R\&D)

laboratories in your firm

\begin{tabular}{cll}
\multicolumn{3}{c}{ Degree of Importance } \\
\hline High $\quad$ Medium Low $\quad \begin{array}{l}\text { Not } \\
\text { relevant }\end{array}$
\end{tabular}

\section{Market sources}

$5 \bigcirc \quad 3 \bigcirc \quad 1 \bigcirc \quad 0 \bigcirc$

$2506 \mathrm{f}$ Suppliers of equipment, materials, components, or software

\begin{tabular}{|c|c|c|c|c|c|c|c|}
\hline 2507 & $\mathrm{~g}$ & Clients or customers & $5 \bigcirc$ & $3 \bigcirc$ & 10 & $0 \bigcirc$ & \\
\hline 2508 & $\mathrm{~h}$ & $\begin{array}{l}\text { Competitors or other firms in } \\
\text { your sector }\end{array}$ & $5 \bigcirc$ & $3 \bigcirc$ & $1 \bigcirc$ & $0 \bigcirc$ & \\
\hline 2509 & $\mathrm{i}$ & Consultants & $5 \bigcirc$ & $3 \bigcirc$ & $1 \bigcirc$ & $0 \bigcirc$ & \\
\hline \multirow[t]{4}{*}{2510} & j & Commercial labs/R\&D firms & $5 \bigcirc$ & $3 \bigcirc$ & $1 \bigcirc$ & $0 \bigcirc$ & \\
\hline & & & \multicolumn{4}{|c|}{ Degree of Importance } & \\
\hline & & & High & Medium & Low & $\begin{array}{l}\text { Not } \\
\text { relevant }\end{array}$ & \\
\hline & & Institutional sources & & & & & S_PUBLIC \\
\hline 2511 & $\mathrm{k}$ & $\begin{array}{l}\text { Universities or other higher } \\
\text { education } \\
\text { institutions }\end{array}$ & $5 \bigcirc$ & $3 \bigcirc$ & 10 & $0 \bigcirc$ & \\
\hline 2512 & I & Colleges/technical institutes & $5 \bigcirc$ & $3 \bigcirc$ & $1 \bigcirc$ & $0 \bigcirc$ & \\
\hline 2513 & $\mathrm{~m}$ & Federal government & $5 \bigcirc$ & $3 \bigcirc$ & $1 \bigcirc$ & $0 \bigcirc$ & \\
\hline
\end{tabular}




\begin{tabular}{|c|c|c|c|c|c|c|}
\hline 2514 & $\mathrm{n}$ & $\begin{array}{l}\text { Provincial/territorial } \\
\text { government research } \\
\text { laboratories }\end{array}$ & $5 \bigcirc$ & $3 \bigcirc$ & $1 \bigcirc$ & $0 \bigcirc$ \\
\hline \multirow[t]{3}{*}{2515} & 0 & $\begin{array}{l}\text { Private non-profit research } \\
\text { laboratories }\end{array}$ & $5 \bigcirc$ & $3 \bigcirc$ & $1 \bigcirc$ & $0 \bigcirc$ \\
\hline & & & \multicolumn{4}{|c|}{ Degree of Importance } \\
\hline & & Other sources & High & Medium & Low & $\begin{array}{l}\text { Not } \\
\text { relevant }\end{array}$ \\
\hline 2516 & $\mathrm{p}$ & $\begin{array}{l}\text { Conferences, trade fairs, } \\
\text { exhibitions }\end{array}$ & $5 \bigcirc$ & $3 \bigcirc$ & $1 \bigcirc$ & $0 \bigcirc$ \\
\hline 2517 & $q$ & $\begin{array}{l}\text { Scientific journals and } \\
\text { trade/technical publications }\end{array}$ & $5 \bigcirc$ & $3 \bigcirc$ & $1 \bigcirc$ & $0 \bigcirc$ \\
\hline 2518 & $r$ & $\begin{array}{l}\text { Investors (banks, venture } \\
\text { capitalists, etc.) }\end{array}$ & $5 \bigcirc$ & $3 \bigcirc$ & $1 \bigcirc$ & $0 \bigcirc$ \\
\hline 2519 & $\mathrm{~s}$ & Industry associations & $5 \bigcirc$ & $3 \bigcirc$ & $1 \bigcirc$ & $0 \bigcirc$ \\
\hline 2520 & $\mathrm{t}$ & Internet & $5 \bigcirc$ & $3 \bigcirc$ & $1 \bigcirc$ & $0 \bigcirc$ \\
\hline 2521 & $\mathrm{u}$ & $\begin{array}{l}\text { Experienced risk-takers or } \\
\text { entrepreneurs }\end{array}$ & $5 \bigcirc$ & $3 \bigcirc$ & $1 \bigcirc$ & $0 \bigcirc$ \\
\hline 2522 & $\mathrm{v}$ & Other, please specify & $5 \bigcirc$ & $3 \bigcirc$ & 10 & $0 \bigcirc$ \\
\hline
\end{tabular}

26. During the three years, $\mathbf{2 0 0 2}$ to $\mathbf{2 0 0 4}$, did your plant co-operate on any of your innovation activities with other firms or institutions? Innovation co-operation is active participation with other firms or organizations on innovation activities. Exclude pure contracting out of work where there is no active co- operation.

$\begin{array}{llllll}2600 & 1 & \bigcirc & \text { Yes } & & \text { COOP } \\ & 3 & \bigcirc & \mathrm{No} & \text { Please go to Question } 29 & \end{array}$

If yes, please check which of the following reasons were important in determining the involvement of your business unit in co-operative arrangements to develop or commercialize new or significantly improved products (goods or services) and/or processes.

\section{Development of innovation}

2601 a Sharing the cost of developing innovations

2602 b Accessing research and development (R\&D)

2603 c Accessing critical expertise

2604 d Prototype development

2605 e Scaling up production processes

Commercialization of innovation

2606 f Accessing new markets

2607 g Accessing new distribution channels

Other
2608 h
Other reasons, please specify
Yes No

$1 \bigcirc \quad 3 \bigcirc$

$1 \bigcirc 3 \bigcirc$

$1 \bigcirc 3 \bigcirc$

$1 \bigcirc 3 \bigcirc$

$1 \bigcirc 3 \bigcirc$

$1 \bigcirc 3 \bigcirc$

$1 \bigcirc 3 \bigcirc$

$1 \bigcirc 3 \bigcirc$ 
27. Please indicate the type of co-operation partner and their geographical location during the three years 2002 to 2004 . (Check all that apply)

$\begin{array}{cccc}\begin{array}{c}\text { Type of co-operation In your In the US } \\ \text { provincerest of }\end{array} & \text { Mexico Europe } & \begin{array}{l}\text { Asia Other Not } \\ \text { partner }\end{array} \\ \text { or } & & \text { Pacificcountriesrelevant }\end{array}$

\section{territory}

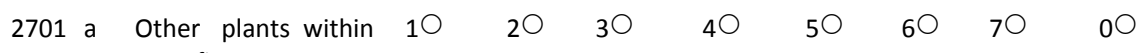
your firm

$\begin{array}{llllllllll}2702 \text { b Suppliers } & \text { of } 1 \bigcirc & 2 \bigcirc & 3 \bigcirc & 4 \bigcirc & 5 \bigcirc & 6 \bigcirc & 7 \bigcirc & 0\end{array}$ equipment, materials, components, or software

\begin{tabular}{|c|c|c|c|c|c|c|c|c|c|c|}
\hline 2703 & $c$ & $\begin{array}{l}\text { Clients } \\
\text { customers }\end{array}$ & $1 \bigcirc$ & $2 \bigcirc$ & $3 \bigcirc$ & $4 \bigcirc$ & $5 \bigcirc$ & 60 & $7 \bigcirc$ & $0 \bigcirc$ \\
\hline 2704 & $d$ & $\begin{array}{l}\text { Competitors or } \\
\text { other firms in your } \\
\text { sector }\end{array}$ & $1 \bigcirc$ & $2 \bigcirc$ & $3 \bigcirc$ & $4 \bigcirc$ & $5 \bigcirc$ & $6 \bigcirc$ & $7 \bigcirc$ & $0 \bigcirc$ \\
\hline 2705 & e & Consultants & $1 \bigcirc$ & $2 \bigcirc$ & $3 \bigcirc$ & $4 \bigcirc$ & $5 \bigcirc$ & 60 & $7 \bigcirc$ & $0 \bigcirc$ \\
\hline \multirow[t]{2}{*}{2706} & $f$ & $\begin{array}{l}\text { Commercial } \\
\text { labs/R\&D }\end{array}$ & $1 \bigcirc$ & $2 \bigcirc$ & $3 \bigcirc$ & $4 \bigcirc$ & $5 \bigcirc$ & $6 \bigcirc$ & $7 \bigcirc$ & $0 \bigcirc$ \\
\hline & & enterprises & & & & & & & & \\
\hline 2707 & g & $\begin{array}{lr}\text { Universities } & \text { or } \\
\text { other } & \text { higher } \\
\text { education } & \\
\text { institutions } & \end{array}$ & $1 \bigcirc$ & $2 \bigcirc$ & $3 \bigcirc$ & $4 \bigcirc$ & $5 \bigcirc$ & $6 \bigcirc$ & $7 \bigcirc$ & $0 \bigcirc$ \\
\hline 2708 & $\mathrm{~h}$ & $\begin{array}{l}\text { Colleges/technical } \\
\text { institutes }\end{array}$ & $1 \bigcirc$ & $2 \bigcirc$ & $3 \bigcirc$ & $4 \bigcirc$ & $5 \bigcirc$ & $6 \bigcirc$ & $7 \bigcirc$ & $0 \bigcirc$ \\
\hline 2709 & $\mathrm{i}$ & $\begin{array}{l}\text { Federal } \\
\text { government } \\
\text { laboratories } \\
\text { research institutes }\end{array}$ & $1 \bigcirc$ & $2 \bigcirc$ & $3 \bigcirc$ & $4 \bigcirc$ & $5 \bigcirc$ & 60 & $7 \bigcirc$ & $0 \bigcirc$ \\
\hline 2710 & j & $\begin{array}{l}\text { Provincial/territorial } \\
\text { government } \\
\text { laboratories } \\
\text { research institutes }\end{array}$ & $1 \bigcirc$ & $2 \bigcirc$ & $3 \bigcirc$ & $4 \bigcirc$ & $5 \bigcirc$ & $6 \bigcirc$ & $7 \bigcirc$ & $0 \bigcirc$ \\
\hline 2711 & k & $\begin{array}{l}\text { Private non-profit } \\
\text { research institutes }\end{array}$ & $1 \bigcirc$ & $2 \bigcirc$ & $3 \bigcirc$ & $4 \bigcirc$ & $5 \bigcirc$ & 60 & $7 \bigcirc$ & $0 \bigcirc$ \\
\hline 2712 & I & $\begin{array}{l}\text { Industrial } \\
\text { associations }\end{array}$ & 10 & $2 \bigcirc$ & $3 \bigcirc$ & $4 \bigcirc$ & $5 \bigcirc$ & $6 \bigcirc$ & $7 \bigcirc$ & $0 \bigcirc$ \\
\hline \multirow[t]{2}{*}{2713} & $\mathrm{~m}$ & $\begin{array}{l}\text { Other types of } \\
\text { partners }\end{array}$ & $1 \bigcirc$ & $2 \bigcirc$ & $3 \bigcirc$ & $4 \bigcirc$ & $5 \bigcirc$ & $6 \bigcirc$ & $7 \bigcirc$ & $0 \bigcirc$ \\
\hline & & Please specify & & & & & & & & \\
\hline
\end{tabular}


28 Which type of co-operation partner did you find the most valuable for your plant's innovation activities?

2801

(Give corresponding letter)

\section{Impact of innovation}

29. Please indicate the degree of importance of each of the following impacts of your product (good or service) and/or process innovationsintroduced during the three years, 2002 to 2004.

Impacts of innovation

Product oriented impacts

2901 a Increased range of goods or

2902 b Improved quality of goods or services

Process orientedimpacts

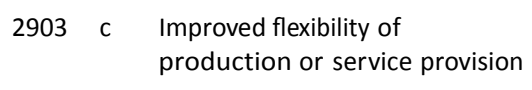

2904 d Increased the speed of supplying and/or delivering goods or services

2905 e Reduced labour costs per un output

$2906 \mathrm{f} \quad$ Increased capability of production or service provision

2907 g Reduced materials or energy per unit output

Impacts on plant

2908 h Increased the plant's productivity

2909 i Allowed plant's expansion

$2910 \mathrm{j} \quad$ Improved the quality of jobs

\section{Market impacts}

2911 k Allowed expansion to new markets

\section{2 | Increased the plant's} profitability

$2913 \mathrm{~m}$ Increased market share

$2914 \mathrm{n} \quad$ Allowed plant to maintain its profit margins
Degree of Importance

\begin{tabular}{lll}
\hline High Medium Low & $\begin{array}{l}\text { Not } \\
\text { relevant }\end{array}$
\end{tabular}

$\begin{array}{llll}5 & 3 & & \end{array}$

$5 \bigcirc \quad 3 \bigcirc \quad 1 \bigcirc \quad 0 \bigcirc$

Degree of Importance

\begin{tabular}{lll}
\hline High Medium Low & $\begin{array}{l}\text { Not } \\
\text { relevant }\end{array}$
\end{tabular}

$\begin{array}{llll}5 \bigcirc & 3 & 1 & 0\end{array}$

$5 \bigcirc \quad 3 \bigcirc \quad 1 \bigcirc \quad 0 \bigcirc$

$\begin{array}{cccc}5 \bigcirc & 3 \bigcirc & 1 \bigcirc & 0 \bigcirc \\ 5 \bigcirc & 3 \bigcirc & 1 \bigcirc & 0 \bigcirc \\ 5 \bigcirc & 3 \bigcirc & 1 \bigcirc & 0 \bigcirc\end{array}$

\section{Degree of Importance}

\begin{tabular}{llcl}
\hline High & Medium & Low & $\begin{array}{l}\text { Not } \\
\text { relevant } \\
5 \bigcirc\end{array}$ \\
& $3 \bigcirc$ & $1 \bigcirc$ & $0 \bigcirc$ \\
$5 \bigcirc$ & $3 \bigcirc$ & $1 \bigcirc$ & $0 \bigcirc$ \\
$5 \bigcirc$ & $3 \bigcirc$ & $1 \bigcirc$ & $0 \bigcirc$ \\
& Degree of Importance & \\
\hline High & Medium & Low & $\begin{array}{l}\text { Not } \\
\text { relevant }\end{array}$
\end{tabular}

$\begin{array}{llll}5 \bigcirc & 3 \bigcirc & 1 \bigcirc & 0 \bigcirc \\ 5 \bigcirc & 3 \bigcirc & 1 \bigcirc & 0 \bigcirc \\ 5 \bigcirc & 3 \bigcirc & 1 \bigcirc & 0 \bigcirc \\ 5 \bigcirc & 3 \bigcirc & 1 \bigcirc & 0 \bigcirc\end{array}$




\begin{tabular}{|c|c|c|c|c|c|c|}
\hline 2915 & 0 & $\begin{array}{l}\text { Allowed the plant to keep up } \\
\text { with its competitors }\end{array}$ & $5 \bigcirc$ & $3 \bigcirc$ & $1 \bigcirc$ & $0 \bigcirc$ \\
\hline 2916 & $\mathrm{p}$ & Increased market visibility & $5 \bigcirc$ & $3 \bigcirc$ & $1 \bigcirc$ & $0 \bigcirc$ \\
\hline \multirow[t]{3}{*}{2917} & $q$ & $\begin{array}{l}\text { Met requirements of existing } \\
\text { clients }\end{array}$ & & & & \\
\hline & & & \multicolumn{4}{|c|}{ Degree of Importance } \\
\hline & & Other impacts & High & Medium & Low & $\begin{array}{l}\text { Not } \\
\text { relevant }\end{array}$ \\
\hline 2918 & r & $\begin{array}{l}\text { Reduced environmental } \\
\text { impacts }\end{array}$ & $5 \bigcirc$ & $3 \bigcirc$ & $1 \bigcirc$ & $0 \bigcirc$ \\
\hline 2919 & s & Improved health and safety & $5 \bigcirc$ & $3 \bigcirc$ & $1 \bigcirc$ & $0 \bigcirc$ \\
\hline 2920 & $\mathrm{t}$ & Met regulatory requirements & $5 \bigcirc$ & $3 \bigcirc$ & $1 \bigcirc$ & $0 \bigcirc$ \\
\hline 2921 & u & Other, please specify & $5 \bigcirc$ & $3 \bigcirc$ & $1 \bigcirc$ & $0 \bigcirc$ \\
\hline
\end{tabular}

\section{Problems and obstacles}

30. During the three years, $\mathbf{2 0 0 2}$ to $\mathbf{2 0 0 4}$, how important were the following problems and obstacles for slowing down or causing problems for your innovation activities or innovation projects?

\section{Problems and obstacles: development of innovation}

3001 a Lack of funds within your plan or your firm for innovation

3002 b Lack of finance from sources outside your plant for innovation

3003 c Innovation costs too high

3004 d Lack of qualified personnel to work on innovation projects

3005 e Lack of information on 5 technology
$3006 \mathrm{f}$ Difficulty in finding cooperation 5 partners for innovation

3007 g Inability to devote staff to innovation projects on an ongoing basis because of production requirements

$3008 \mathrm{~h}$ Risk related to the feasibility of the innovation project

3009 i Other, please specify
Degree of Importance

\begin{tabular}{lll}
\hline High Medium Low & $\begin{array}{l}\text { Not } \\
\text { relevant }\end{array}$
\end{tabular}

$\begin{array}{llll}5 \bigcirc & 3 \bigcirc & 1 & 0\end{array}$

$5 \bigcirc \quad 3 \bigcirc \quad 1 \bigcirc \quad 0 \bigcirc$

$5 \bigcirc \quad 3 \bigcirc \quad 10 \quad 0 \bigcirc$

$5 \bigcirc \quad 3 \bigcirc \quad 1 \bigcirc \quad 0 \bigcirc$

$5 \bigcirc \quad 3 \bigcirc \quad 1 \bigcirc \quad 0 \bigcirc$

$5 \bigcirc \quad 3 \bigcirc \quad 1 \bigcirc \quad 0 \bigcirc$

$5 \bigcirc \quad 3 \bigcirc \quad 1 \bigcirc \quad 0 \bigcirc$

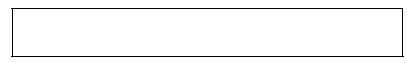

\section{.}

$\bigcirc$




\begin{tabular}{|c|c|c|c|c|c|c|}
\hline \multirow[b]{3}{*}{3010} & \multirow[b]{3}{*}{ j } & \multirow[b]{2}{*}{$\begin{array}{l}\text { Problems and obstacles: } \\
\text { commercialization of } \\
\text { innovation }\end{array}$} & \multicolumn{4}{|c|}{ Degree of Importance } \\
\hline & & & High & Medium & Low & $\begin{array}{l}\text { Not } \\
\text { relevant }\end{array}$ \\
\hline & & $\begin{array}{l}\text { Market dominated by } \\
\text { established firms }\end{array}$ & $5 \bigcirc$ & $3 \bigcirc$ & 10 & $0 \bigcirc$ \\
\hline 3011 & $\mathrm{k}$ & $\begin{array}{l}\text { Uncertain demand for innovative } \\
\text { goods or services }\end{array}$ & $5 \bigcirc$ & $3 \bigcirc$ & $1 \bigcirc$ & $0 \bigcirc$ \\
\hline 3012 & I & Lack of knowledge of markets & $5 \bigcirc$ & $3 \bigcirc$ & $1 \bigcirc$ & $0 \bigcirc$ \\
\hline 3013 & $\mathrm{~m}$ & Insufficient marketing effort & $5 \bigcirc$ & $3 \bigcirc$ & 10 & $0 \bigcirc$ \\
\hline 3014 & $\mathrm{n}$ & Inappropriate targeting & $5 \bigcirc$ & $3 \bigcirc$ & $1 \bigcirc$ & $0 \bigcirc$ \\
\hline 3015 & o & Inappropriate packaging & $5 \bigcirc$ & $3 \bigcirc$ & $1 \bigcirc$ & $0 \bigcirc$ \\
\hline 3016 & $\mathrm{p}$ & Lack of consumer acceptance & $5 \bigcirc$ & $3 \bigcirc$ & $1 \bigcirc$ & $0 \bigcirc$ \\
\hline 3017 & $q$ & $\begin{array}{l}\text { Lack of industry-wide } \\
\text { standards }\end{array}$ & $5 \bigcirc$ & $3 \bigcirc$ & $1 \bigcirc$ & $0 \bigcirc$ \\
\hline 3018 & $r$ & $\begin{array}{l}\text { Lack of government standards } \\
\text { and regulations }\end{array}$ & $5 \bigcirc$ & $3 \bigcirc$ & $1 \bigcirc$ & $0 \bigcirc$ \\
\hline 3019 & $\mathrm{~s}$ & Other, please specify: & $5 \bigcirc$ & $3 \bigcirc$ & $1 \bigcirc$ & $0 \bigcirc$ \\
\hline
\end{tabular}

\section{Intellectual property and acquisition of technology}

31. Please indicate which of the following methods were used by your plant $t$ protect its intellectual property during the three years, 2002 to 2004.

Yes No

\section{Formal methods}

\begin{tabular}{|c|c|c|c|c|c|}
\hline 3101 & $\mathrm{a}$ & Patents & $1 \bigcirc$ & $3 \bigcirc$ & METHOD_INST \\
\hline 3102 & $\mathrm{~b}$ & Trademark & $1 \bigcirc$ & $3 \bigcirc$ & METHOD_INST \\
\hline 3103 & $\mathrm{C}$ & Copyrights & $1 \bigcirc$ & $3 \bigcirc$ & METHOD_INST \\
\hline \multirow[t]{2}{*}{3104} & $d$ & Confidentiality agreements & 10 & $3 \bigcirc$ & METHOD_PRIV \\
\hline & & Strategic methods & & & \\
\hline 3105 & $\mathrm{e}$ & Secrecy & $1 \bigcirc$ & $3 \bigcirc$ & METHOD_PRIV \\
\hline 3106 & $f$ & Complexity of design & $1 \bigcirc$ & $3 \bigcirc$ & METHOD_STRA \\
\hline \multirow[t]{2}{*}{3107} & g & Lead-time advantage on competitors & $1 \bigcirc$ & $3 \bigcirc$ & METHOD_STRA \\
\hline & & Other & & & \\
\hline 3108 & $\mathrm{~h}$ & Other, please specify: & $1 \bigcirc$ & $3 \bigcirc$ & \\
\hline
\end{tabular}

32. Please estimate (as best you can) the percentage of your products (goods or services) that were protected by patents (in terms of their contribution to total revenue) in $\mathbf{2 0 0 4}$. 
33. Please estimate (as best you can) the percentage of your products (goods or services) that were protected by trademarks (in terms of their contribution to total revenue) in $\mathbf{2 0 0 4}$.

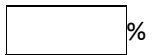

34. During the three years, 2002 to 2004 , did your plant apply for a patent?

$\begin{array}{cccc}3401 & 1 & \bigcirc & \text { Yes } \\ & 3 & \bigcirc & \text { No }\end{array}$

35. During the three years, $\underline{2002}$ to 2004, did your plant acquire licenses from other firms or organizations?

(Please exclude software licenses under $\$ 1,000)$

$\begin{array}{lllll}3500 & 1 & \bigcirc & \text { Yes } & \\ & 3 & \bigcirc & \text { No } & \text { Please go to question } 36\end{array}$

If yes, please indicate the source of the licenses:

\begin{tabular}{llllc} 
& & & Yes & No \\
3501 & a & A Canadian firm & $1 \bigcirc$ & $3 \bigcirc$ \\
3502 & b & A foreign firm & $1 \bigcirc$ & $3 \bigcirc$ \\
3503 & c & A Canadian university & $1 \bigcirc$ & $3 \bigcirc$ \\
3504 & d & A Canadian hospital & $1 \bigcirc$ & $3 \bigcirc$ \\
3505 & e & A Canadian federal government lab & $1 \bigcirc$ & $3 \bigcirc$ \\
3506 & f & A provincial/territorial government lab & $1 \bigcirc$ & $3 \bigcirc$ \\
3507 & g & Other, please specify & $1 \bigcirc$ & $3 \bigcirc$ \\
\cline { 2 - 3 } & & &
\end{tabular}

\section{Market and supply chain}

36. Please estimate (as best you can) the percentage of the total revenue of your plant in $\underline{\mathbf{2 0 0 4}}$ which came from the sale of products (goods or services) to the following industries:

\section{Percentage of total revenue}

\section{NATURAL RESOURCE INDUSTRIES}

3601 a Forestry and logging

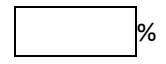

3602 b Wood products manufacturers

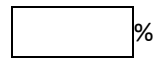

3603 c Pulp and paper products manufacturers

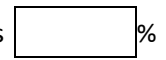

3604 d Mining

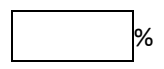

3605 e

Oil and gas extraction

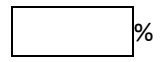




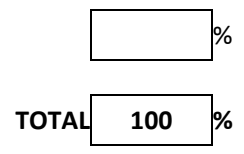

37. Please estimate (as best you can) the percentage of your plant's total expenditures on raw materials and components that were supplied from the different geographical locations listed below in 2004.

(Include suppliers that are part of your larger firm)

$\begin{array}{lll}3701 \text { a } & \text { In your province/territory } \\ 3702 \text { b } & \text { In the rest of Canada } \\ 3703 \text { c } & \text { United States } \\ 3704 \text { d } & \text { Mexico } \\ 3705 \text { e } & \text { Europe } \\ 3706 \text { f } & \text { Asia Pacific } \\ 3707 \text { g } & \text { All other countries }\end{array}$

Percentage of total expenditures

on raw materials and components

3707 g All other countries
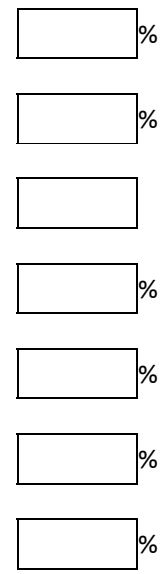

TOTAL $100 \%$

38. In 2004, did your plant buy new machinery or equipment?

If yes, please estimate (as best you can) the percentage of all your plant's expenditures on new machinery and equipment that were supplied from the following different geographical locations in $\mathbf{2 0 0 4}$.

(Include suppliers that are part of your larger firm)

Percentage of total expenditures on machinery and equipment

3801 a In your province/territory

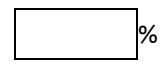

3802 b In the rest of Canada

3803 c United States
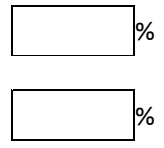

3804 d Mexico

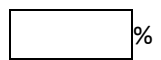




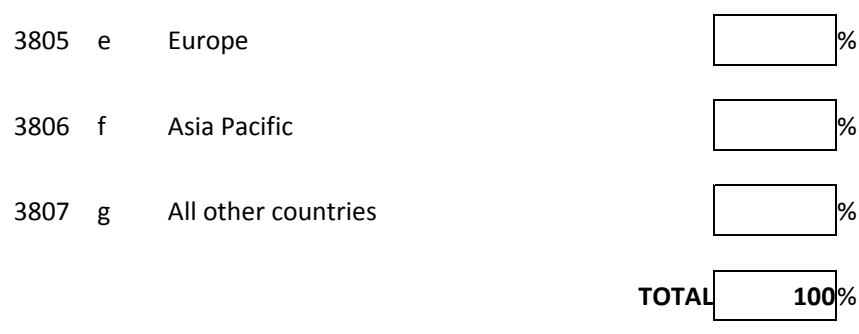

39. In 2004, did your plant contract-out for R\&D services? (Do not include $R \& D$ carried out on your behalf by other plants and $R \& D$ units in your
larger firm)

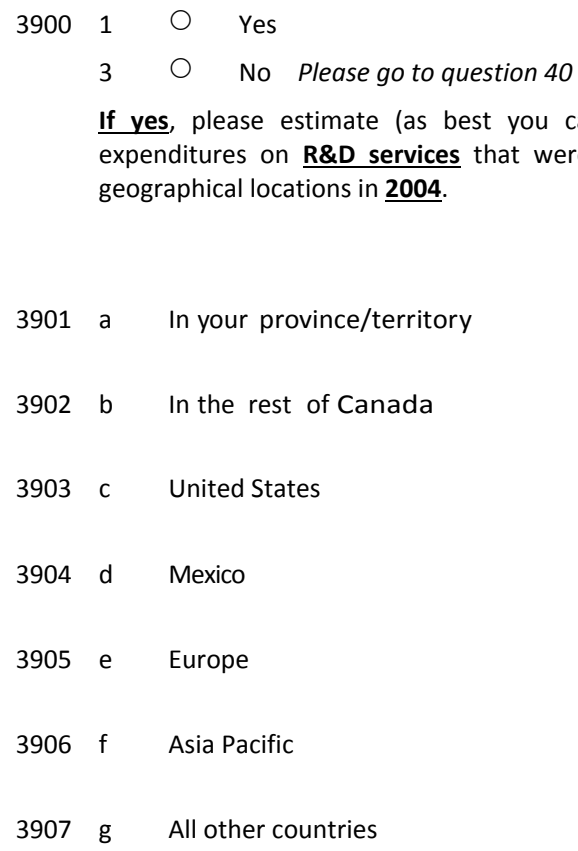
geographical locations in $\mathbf{2 0 0 4}$.

3901 a In your province/territory

3902 b In the rest of Canada

3903 c United States

3904 d Mexico

3905 e Europe

3906 Asia Pacific

3907 g All other countries

If yes, please estimate (as best you can) the percentage of all your plant's expenditures on $\underline{R} \& \mathbf{D}$ services that were supplied from the following different

\section{Percentage of total expenditures on R\&D services}
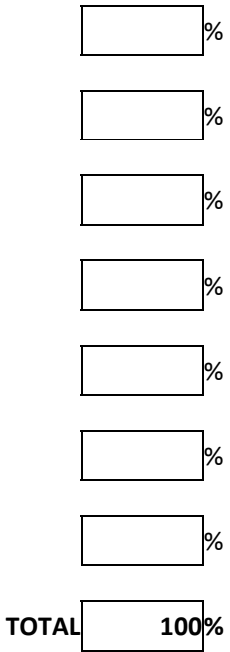

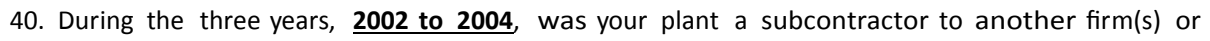
organization(s)?

(Include other plants within your larger firm)

$\begin{array}{llll}4000 & 1 & \bigcirc & \text { Yes } \\ & 3 & \bigcirc & \text { No Please go to question } 41\end{array}$ 
If yes, please estimate (as best you can) the percentage of the total value of production of your plant that was generated through work carried out under subcontracting arrangements with another firm(s) or organization(s), during the three years, 2002 to 2004.

$\begin{array}{lllll}4001 & \text { a } & 2004 & \text { Percentage } \\ 4002 & \text { b } & 2003 & \\ 4003 & \text { c } & 2002 & \\ \end{array}$

\section{Funding and support}

41. During the last three years, $\mathbf{2 0 0 2}$ to 2004, did your plant receive external funding?

$\begin{array}{llll}4100 & 1 & \bigcirc & \text { Yes } \\ & 3 & \bigcirc & \text { No Please go to question } 42\end{array}$

If yes, please indicate which external sources provided funding for your plant during the three years, 2002 to 2004.

\begin{tabular}{lllll}
4101 & a & Conventional sources (i.e. banks) & $1 \bigcirc$ & $3 \bigcirc$ \\
4102 & b & Canadian based venture capital & $1 \bigcirc$ & $3 \bigcirc$ \\
4103 & c & American based venture capital & $1 \bigcirc$ & $3 \bigcirc$ \\
4104 & d & Venture capital from other countries & $1 \bigcirc$ & $3 \bigcirc$ \\
4105 & e & Angel investors/family & $1 \bigcirc$ & $3 \bigcirc$ \\
4106 & f & Private placement & $1 \bigcirc$ & $3 \bigcirc$ \\
4107 & g & IPO (Initial Public Offering) & $1 \bigcirc$ & $3 \bigcirc$ \\
4108 & h & SPO (Secondary Public Offering) & $1 \bigcirc$ & $3 \bigcirc$ \\
4109 & i & Collaborative arrangements, alliances & $1 \bigcirc$ & $3 \bigcirc$ \\
4110 & j & Other, please specify & $1 \bigcirc$ & $3 \bigcirc$ \\
& & \multicolumn{2}{l}{} &
\end{tabular}

42. Did your firm use any of the following types of programs sponsored by the federal ${ }_{\text {RECIP }}$ government or provincial/territorial governments during the three years, 2002 to 2004?

\section{Government Programs}

$\begin{array}{ll}\text { Federal Provincial/Territorial } & \text { Did not use } \\ \text { GovernmentGovernment } & \begin{array}{l}\text { govent } \\ \text { program }\end{array}\end{array}$

Research and

4201 a development (R\&D) tax

10

30

$0 \bigcirc$

$4202 b$

credits

10

30

$0 \bigcirc$ 


\begin{tabular}{|c|c|c|c|}
\hline $4203 \mathrm{c}$ & $\begin{array}{l}\text { Government venture } \\
\text { capital support }\end{array}$ & 10 & $3 \bigcirc$ \\
\hline $4204 d$ & $\begin{array}{l}\text { Government technology } \\
\text { support and assistance } \\
\text { programs }\end{array}$ & 10 & $3 \bigcirc$ \\
\hline 4205 e & Government information & 10 & $3 \bigcirc$ \\
\hline $4206 \mathrm{f}$ & $\begin{array}{l}\text { Government support for } \\
\text { training }\end{array}$ & 10 & $3 \bigcirc$ \\
\hline $4207 \mathrm{~g}$ & Other, please specify: & 10 & $3 \bigcirc$ \\
\hline
\end{tabular}

\section{Adapted from: $\quad$ Statistics Canada, Innovation of Survey 2005 Questionnaire.}

Note: Variable names correspond to the nomenclature used in the OECD microdata project (see CIS IV questionnaire in Appendix 8.E ), with exceptions. 


\section{E. CIS 4 Questionnaire and Corresponding Variables}

\section{General information about the enterprise}

Name of enterprise

Address $^{51}$

Postal code

Main activity

ID

NUTS

NACE

\subsection{Is your enterprise part of an enterprise group? GP}

(A group consists of two or more legally defined enterprises under common ownership. Each enterprise in the group may serve different markets, as with national or regional subsidiaries, or serve different product markets. The head office is also part of an enterprise group.)

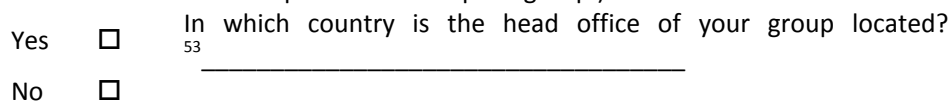

If your enterprise is part of an enterprise group, please answer all further questions only for your enterprise in [your country]. Do not include results for subsidiaries or parent enterprises outside of [your country]

1.2 In which geographic markets did your enterprise sell goods or services during the three years 2002 to 2004 ?

\begin{tabular}{|c|c|c|c|}
\hline & Yes & No & \\
\hline Local / regional within [your country] & $\square$ & $\square$ & MARLOC \\
\hline National & $\square$ & $\square$ & MARNAT \\
\hline Other European Union (EU) countries, EFTA, or EU candidate countries* & $\square$ & $\square$ & MAREUR \\
\hline All other countries & $\square$ & $\square$ & MAROTH \\
\hline
\end{tabular}

A product innovation is the market introduction of a new good or service or a significantly improved good or service with respect to its capabilities, such as improved software, user friendliness, components or subsystems. The innovation (new or improved) must be new to your enterprise, but it does not need to be new to your sector or market. It does not matter if the innovation was originally developed by your enterprise or by other enterprises.

\footnotetext{
${ }^{51}$ NUTS 2 code.

52 NACE 4 digit code.

${ }^{53}$ Country code according to ISO standard.
} 
2.1 During the three years 2002 to 2004 , did your enterprise introduce:

New or significantly improved goods. (Exclude the simple resale of new goods purchased from other enterprises and changes of a solely aesthetic nature.)

New or significantly improved services.

$\square \quad \square \quad I N P D G D$

$\square \quad \square \quad$ INPDSV

If no to both options, go to question 3.1, otherwise:

2.2 Who developed these product innovations?

INPDTW

Select the most appropriate option only

Mainly your enterprise or enterprise group

Your enterprise together with other enterprises or institutions

Mainly other enterprises or institutions

2.3 Were any of your goods and service innovations during the three years 2002 to 2004:

Yes No

New to Your enterprise introduced a new or significantly improved your good or service onto your market before your competitors (it market? may have already been available in other markets)

$\square \quad \square \quad N E W M K T$

Only new Your enterprise introduced a new or significantly improved to your good or service that was already available from your firm? competitors in your market

Using the definitions above, please give the percentage of your total turnover ${ }^{54}$ in 2004 from:

Goods and service innovations introduced during 2002 to 2004 that were new to your market

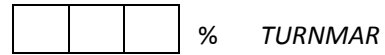

Goods and service innovations introduced during 2002 to 2004 that were only new to your firm

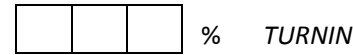

Goods and services that were unchanged or only marginally modified during 2002 to 2004 (include the resale of new goods or services purchased from other enterprises)

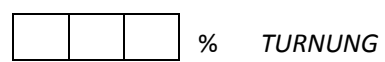

Total turnover in 2004

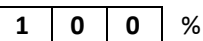

3. Process innovation

A process innovation is the implementation of a new or significantly improved production process, distribution method, or support activity for your goods or services. The innovation (new or improved) must be new to your enterprise, but it does not need to be new to your sector or market. It does not matter if the innovation was originally developed by your enterprise or by other enterprises. Exclude purely organisational innovations.

\footnotetext{
${ }^{54}$ For Credit institutions: Interests receivable and similar income, for insurance services: Gross premiums written.
} 
3.1 During the three years 2002 to 2004 , did your enterprise introduce:

Yes No

New or significantly improved methods of manufacturing or producing goods or services

INPSPD

New or significantly improved logistics, delivery or distribution methods for your inputs, goods or services

INPSLG

New or significantly improved supporting activities for your processes, such as maintenance systems or operations for purchasing, accounting, or computing

If no to all options, go to Section 4, otherwise:

3.2 Who developed these process innovations?

INPCSW

Select the most appropriate option only

Mainly your enterprise or enterprise group

Your enterprise together with other enterprises or institutions

Mainly other enterprises or institutions

\section{Ongoing or abandoned innovation activities}

Innovation activities include the acquisition of machinery, equipment, software, and licenses; engineering and development work, training, marketing and $R \& D^{55}$ when they are specifically undertaken to develop and/or implement a product or process innovation.

4.1 Did your enterprise have any innovation activities to develop product or process innovations that were abandoned during $\mathbf{2 0 0 2}$ to 2004 or still ongoing by the end of 2004 ?

Yes

INONAB

No

If your enterprise had no product or process innovations or innovation activity during 2002 to 2004 (no to all options in questions 2.1, 3.1, and 4.1), go to question 8.2.

Otherwise, go to question 5.1

\section{Innovation activities and expenditures}

5.1 During the three years 2002 to 2004 , did your enterprise engage in the following innovation activities:

\section{Yes No}

Creative work undertaken within your enterprise to

Intramural (in-house) R\&D increase the stock of knowledge and its use to devise new and improved products and processes (including software

RRDIN development)

If yes, did your firm perform R\&D during 2002 to 2004 :

RDENG

\footnotetext{
${ }^{55}$ Include basic R\&D as an innovation activity even if not specifically related to a product and/or process innovation.
} 
Continuously?

Occasionally?

Extramural R\&D companies (including other enterprises within your group) or by public or private research organisations and purchased by your enterprise

Acquisition of machinery, equipment and software

Acquisition of other external knowledge

Training

Market introduction of innovations

Other preparations
Acquisition of advanced machinery, equipment and computer hardware or software to produce new or significantly improved products and processes

Purchase or licensing of patents and non-patented inventions, know-how, and other types of knowledge from other enterprises or organisations

Internal or external training for your personnel specifically for the development and/or introduction of new or significantly improved products and processes

Activities for the market introduction of your new or significantly improved goods and services, including market research and launch advertising

Procedures and technical preparations to implement new or significantly improved products and processes that are not covered elsewhere

5.2 Please estimate the amount of expenditure for each of the following four innovation activities in 2004 only. (Include personnel and related costs) ${ }^{56}$

Tick 'nil' if your enterprise had no expenditures in $2004 \mathrm{Nil}$

Intramural (in-house) R\&D (Include capital expenditures on buildings and equipment specifically for R\&D)

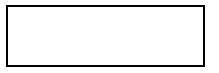

RRDINX

Acquisition of R\&D (extramural R\&D)

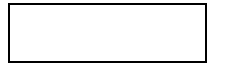

RRDEXX

Acquisition of machinery, equipment and software (Exclude expenditures on equipment for R\&D)

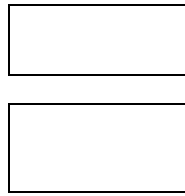

Acquisition of other external knowledge

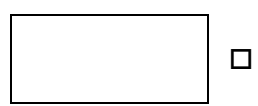

\footnotetext{
${ }^{56}$ Give expenditure data in national currency units to eight digits.
} 
5.3 During the three years $\mathbf{2 0 0 2}$ to 2004 , did your enterprise receive any public financial support for innovation activities from the following levels of government? Include financial support via tax credits or deductions, grants, subsidised loans, and loan guarantees. Exclude research and other innovation activities conducted entirely for the public sector under contract.

Local or regional authorities

Yes No

Central government (including central government agencies or ministries)

FUNLOC

The European Union (EU)

FUNGMT

If yes, did your firm participate in the EU's $5^{\text {th }}(1998-2002)$ or $6^{\text {th }}(2003-2006)$ Framework Programme for Research and Technical Development

FUNEU

FUNRTD

\section{Sources of information and co-operation for innovation activities}

6.1 During the three years 2002 to 2004 , how important to your enterprise's innovation activities were each of the following information sources? Please identify information sources that provided information for new innovation projects or contributed to the completion of existing innovation projects.

Degree of importance

Tick 'not used' if no information was obtained from a source.

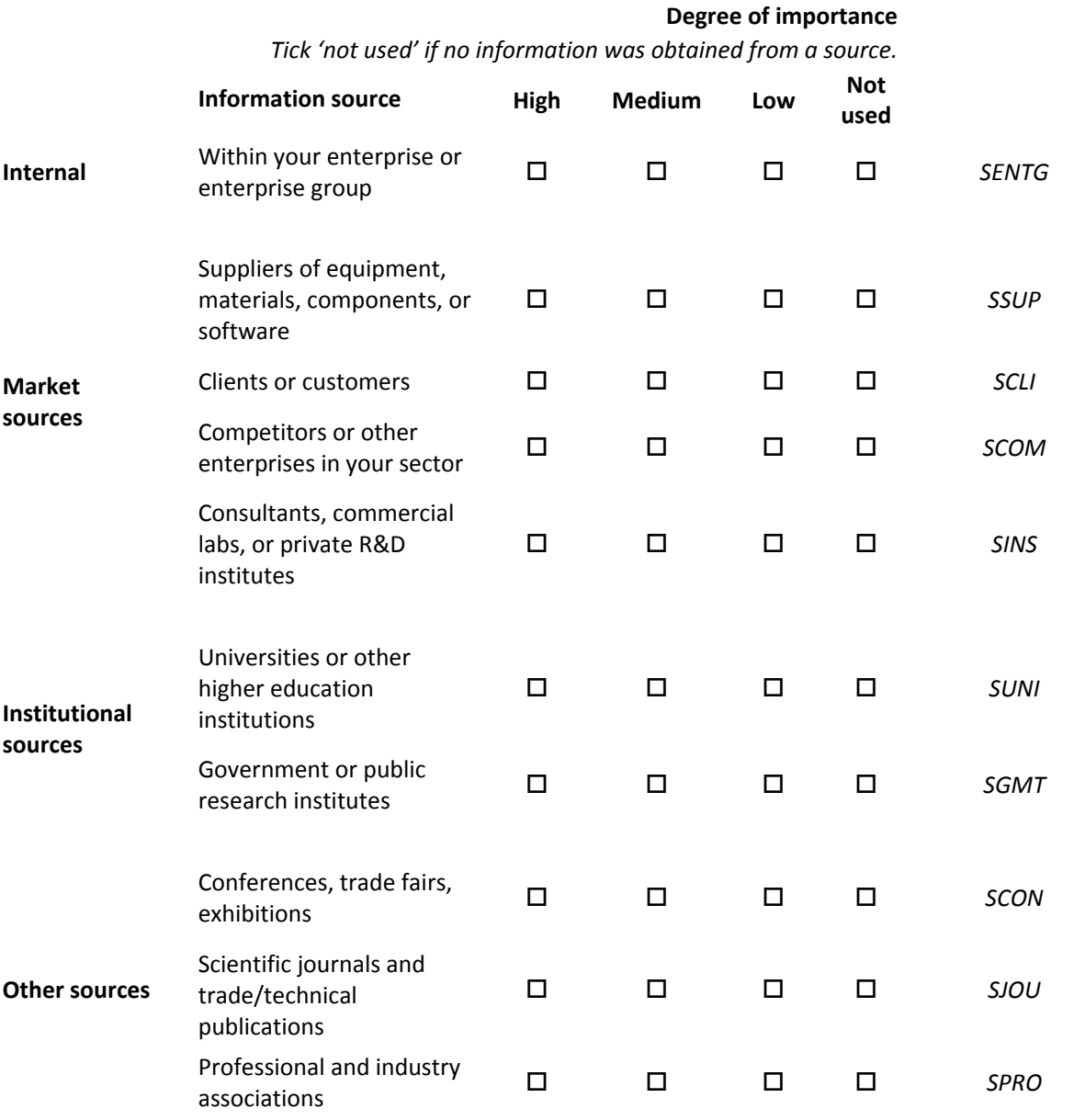


6.2 During the three years 2002 to 2004 , did your enterprise co-operate on any of your innovation activities with other enterprises or institutions? Innovation co-operation is active participation with other enterprises or non-commercial institutions on innovation activities. Both partners do not need to commercially benefit. Exclude pure contracting out of work with no active co-operation.

Yes

No $\quad \square \quad$ (Please go to question 7.1)

\subsection{Please indicate the type of co-operation partner and location}

\section{Type of co-operation partner}

A. Other enterprises within your enterprise group

B. Suppliers of equipment, materials, components, or software

C. Clients or customers

D. Competitors or other enterprises in your sector

E. Consultants, commercial labs, or private R\&D institutes

F. Universities or other higher education institutions

G. Government or public research institutes

$\begin{array}{cccc}\text { [Your } & \text { Other } & \text { United } & \text { All other } \\ \text { country] } & \text { Europe* } & \text { States } & \text { countries }\end{array}$

$\begin{array}{llll}\text { Co11 } & \text { Co12 } & \text { Co13 } & \text { Co14 } \\ \text { Co21 } & \text { Co22 } & \text { Co23 } & \text { Co24 } \\ \text { Co31 } & \text { Co32 } & \text { Co33 } & \text { Co34 } \\ \text { Co41 } & \text { Co42 } & \text { Co43 } & \text { Co44 } \\ \text { Co51 } & \text { Co52 } & \text { Co53 } & \text { Co54 } \\ \text { Co61 } & \text { Co62 } & \text { Co63 } & \text { Co64 } \\ \text { Co71 } & \text { Co72 } & \text { Co73 } & \text { Co } 74\end{array}$

*: Include the following European Union (EU) countries, EFTA, or EU candidate countries: Austria, Belgium, Bulgaria, Croatia, Cyprus, Czech Republic, Denmark, Estonia, Finland, France, Germany, Greece, Hungary, Iceland, Italy, Ireland, Latvia, Liechtenstein, Lithuania, Luxembourg, Malta, Netherlands, Norway, Poland, Portugal, Romania, Slovenia, Slovakia, Switzerland, Turkey, Spain, Sweden and the United Kingdom.

6.4 Which type of co-operation partner did you find the most valuable for your enterprise's innovation activities? (Give corresponding letter) PMOS

\section{Effects of innovation during 2002-2004}

7.1 How important were each of the following effects of your product (good or service) and process innovations introduced during the three years 2002 to 2004 ?

Degree of observed effect

High Medium Low $\begin{gathered}\text { Not } \\ \text { relevant }\end{gathered}$

Increased range of goods or services

ERANGE

Product oriented Entered new markets or increased market share

Improved quality of goods or services 


\begin{tabular}{|c|c|c|c|c|c|c|}
\hline & $\begin{array}{l}\text { Improved flexibility of } \\
\text { production or service } \\
\text { provision }\end{array}$ & $\square$ & $\square$ & $\square$ & $\square$ & EFLEX \\
\hline \multirow{3}{*}{$\begin{array}{l}\text { Process } \\
\text { oriented } \\
\text { effects }\end{array}$} & $\begin{array}{l}\text { Increased capacity of } \\
\text { production or service } \\
\text { provision }\end{array}$ & $\square$ & $\square$ & $\square$ & $\square$ & $E C A P$ \\
\hline & $\begin{array}{l}\text { Reduced labour costs per } \\
\text { unit output }\end{array}$ & $\square$ & $\square$ & $\square$ & $\square$ & $E L B R$ \\
\hline & $\begin{array}{l}\text { Reduced materials and } \\
\text { energy per unit output }\end{array}$ & $\square$ & $\square$ & $\square$ & $\square$ & $E M A T$ \\
\hline \multirow{2}{*}{$\begin{array}{l}\text { Other } \\
\text { effects }\end{array}$} & $\begin{array}{l}\text { Reduced environmental } \\
\text { impacts or improved health } \\
\text { and safety }\end{array}$ & $\square$ & $\square$ & $\square$ & $\square$ & EENV \\
\hline & $\begin{array}{l}\text { Met regulatory } \\
\text { requirements }\end{array}$ & $\square$ & $\square$ & $\square$ & $\square$ & ESTD \\
\hline
\end{tabular}

8. Factors hampering innovation activities

8.1 During the three years 2002 to 2004 , were any of your innovation activities or projects:

$\begin{array}{lccc} & \text { Yes } & \text { No } & \\ \text { Abandoned in the concept stage } & \square & \square & \text { HCON } \\ \text { Abandoned after the activity or project was begun } & \square & \square & \text { HBEG } \\ \text { Seriously delayed } & \square & \square & \text { HDLAY }\end{array}$

TO BE ANSWERED BY ALL ENTERPRISES:

8.2 During the three years 2002 to 2004, how important were the following factors for hampering your innovation activities or projects or influencing a decision not to innovate?

Degree of importance

\begin{tabular}{|c|c|c|c|c|c|c|}
\hline & & High & Medium & Low & $\begin{array}{l}\text { Factor not } \\
\text { experienced }\end{array}$ & \\
\hline \multirow{3}{*}{ Cost factors } & $\begin{array}{l}\text { Lack of funds within } \\
\text { your enterprise or group }\end{array}$ & $\square$ & $\square$ & $\square$ & $\square$ & HFENT \\
\hline & $\begin{array}{l}\text { Lack of finance from } \\
\text { sources outside your } \\
\text { enterprise }\end{array}$ & $\square$ & $\square$ & $\square$ & $\square$ & HFOUT \\
\hline & $\begin{array}{l}\text { Innovation costs too } \\
\text { high }\end{array}$ & $\square$ & $\square$ & $\square$ & $\square$ & HCOS \\
\hline \multirow{4}{*}{$\begin{array}{l}\text { Knowledge } \\
\text { factors }\end{array}$} & $\begin{array}{l}\text { Lack of qualified } \\
\text { personnel }\end{array}$ & $\square$ & $\square$ & $\square$ & $\square$ & HPER \\
\hline & $\begin{array}{l}\text { Lack of information on } \\
\text { technology }\end{array}$ & $\square$ & $\square$ & $\square$ & $\square$ & HTEC \\
\hline & $\begin{array}{l}\text { Lack of information on } \\
\text { markets }\end{array}$ & $\square$ & $\square$ & $\square$ & $\square$ & HINF \\
\hline & $\begin{array}{l}\text { Difficulty in finding } \\
\text { cooperation partners for }\end{array}$ & $\square$ & $\square$ & $\square$ & $\square$ & HPAR \\
\hline
\end{tabular}


innovation

Market dominated by established enterprises

HDOM

Market

Uncertain demand for innovative goods or services

No need due to prior

Reasons not innovations

No need because of no

demand for innovations

$\square \quad \square$

to innovate

\section{Intellectual property rights}

\subsection{During the three years 2002 to 2004 , did your enterprise:}

Apply for a patent

Yes No

Register an industrial design

$\square \quad$ PROPAT

Register a trademark

$\square \quad P R O D S G$

PROTM

Claim copyright

PROCP

\section{Organisational and marketing innovations}

An organisational innovation is the implementation of new or significant changes in firm structure or management methods that are intended to improve your firm's use of knowledge, the quality of your goods and services, or the efficiency of work flows. A marketing innovation is the implementation of new or significantly improved designs or sales methods to increase the appeal of your goods and services or to enter new markets.

10.1 During the three years 2002 to 2004 , did your enterprise introduce:

\begin{tabular}{|c|c|c|c|c|}
\hline & & Yes & No & \\
\hline & $\begin{array}{l}\text { New or significantly improved knowledge } \\
\text { management systems to better use or exchange } \\
\text { information, knowledge and skills within your } \\
\text { enterprise }\end{array}$ & $\square$ & $\square$ & ORGSYS \\
\hline $\begin{array}{l}\text { Organisational } \\
\text { innovations }\end{array}$ & $\begin{array}{l}\text { A major change to the organisation of work within } \\
\text { your enterprise, such as changes in the management } \\
\text { structure or integrating different departments or } \\
\text { activities }\end{array}$ & $\square$ & $\square$ & ORGSTR \\
\hline & $\begin{array}{l}\text { New or significant changes in your relations with } \\
\text { other firms or public institutions, such as through } \\
\text { alliances, partnerships, outsourcing or sub- } \\
\text { contracting }\end{array}$ & $\square$ & $\square$ & ORGREL \\
\hline $\begin{array}{l}\text { Marketing } \\
\text { innovations }\end{array}$ & $\begin{array}{l}\text { Significant changes to the design or packaging of a } \\
\text { good or service (Exclude routine/ seasonal changes } \\
\text { such as clothing fashions) }\end{array}$ & $\square$ & $\square$ & MKTDES \\
\hline
\end{tabular}


New or significantly changed sales or distribution methods, such as internet sales, franchising, direct sales or distribution licenses

10.2 If your enterprise introduced an organisational innovation during the three years 2002 to 2004 , how important were each of the following effects?

\section{Degree of observed effect}

High Medium Low $\begin{gathered}\text { Not } \\ \text { relevant }\end{gathered}$

Reduced time to respond to customer or supplier needs

Improved quality of your goods or services

Reduced costs per unit output

$\begin{array}{lllll}\square & \square & \square & \square & \text { EFORED } \\ \square & \square & \square & \square & \text { EFORQU } \\ \square & \square & \square & \square & \text { EFORCO } \\ \square & \square & \square & \square & \text { EFORSA }\end{array}$

Improved employee satisfaction and/or

reduced rates of employee turnover

\section{Basic economic information on your enterprise}

11.1 What was your enterprise's total turnover for $\mathbf{2 0 0 2}$ and $\mathbf{2 0 0 4 ? ^ { 5 7 }}$ Turnover is defined as the market sales of goods and services (Include all taxes except VAT ${ }^{58}$ ).

2002

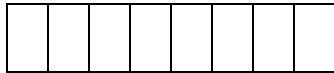

TURNO2
2004

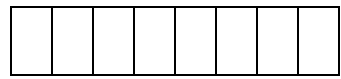

TURNO4

11.2 What was your enterprise's total number of employees in 2002 and $2004 ?^{59}$

2002

2004

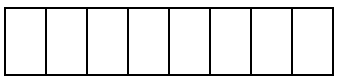

EMPO2

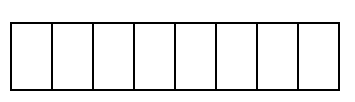

EMP04

Adapted from: OECD, The fourth Community Innovation Survey (CIS IV): The harmonised Questionnaire.

\footnotetext{
${ }^{57}$ Give turnover in '000 of national currency units to nine digits.

${ }^{58}$ For Credit institutions: Interests receivable and similar income; for Insurance services: Gross premiums written.

${ }^{59}$ Annual average. If not available, give the number of employees at the end of each year. Give figures to six digits.
} 

PART III

GENERAL DISCUSSION 

CHAPTER 9

Conclusions 


\subsection{Summary of Key Findings}

This study aimed to empirically assess the effectiveness of direct and indirect measures of government support to R\&D and innovation in Québec on both the private $R \& D$ investment of firms and their economic performance, in particular in terms of labour productivity. These issues were addressed through an examination of the research questions that led to the findings presented below. However, to begin, we present the main messages gathered through the examination of the descriptive statistics in Chapters 5 to 8 regarding the use of tax credits and subsidies by the firms.

First, there is an asymmetric distribution of both types of government incentives between large and small firms. The latter use government incentives more often than large firms, but the greater part of the incentives goes to the former. The same contrast was also found between the high technology group and the medium-low and low technology group. The high technology group, which represents a small percentage of applicants, is the one that receives the largest portion of the funding.

Second, among the fiscal incentives offered to firms, the tax credit for salaries of researchers was the most popular tax credit - challenged for a short period by the superdeductions that were abolished after one year - with amounts of tax credits across all sizes of firms above $75 \%$ of total attributed tax credits.

Empirical analysis and the estimation results were very successful and helped to highlight important policy considerations. The findings are presented through the following research questions:

Research question 1: Does fiscal policy subsidizing salaries and wages of researchers translates into increased ratios of researchers in total employment?

Researchers play a key role in the development of a knowledge-based economy. Since the early 1980s, incentives for R\&D and innovation in Québec have primarily been paid out as salaries to researchers. We examined in Chapter 3 the impact of this policy on the ratio of researchers. A review of statistics on employees assigned to R\&D activities has shown that over the last decade the latter increased at an average rate of $4.7 \%$ in Québec. This increase has not been sufficient to close the gap with Ontario and the Canadian average regarding the proportion of researchers. For example, in 2008, the share of researchers in total employees engaged in R\&D in Québec stood at 57.6\%, while it reached $63.4 \%$ in Ontario and $61.4 \%$ in Canada as a whole. Regarding the distribution of researchers by sector of execution of R\&D, the province of Québec ranked first among Canadian provinces with the highest 
proportion of R\&D personnel in the business sector (70\%) in 2005. However, the gap with other top R\&D performers in Canada was not significant. Indeed, Ontario and British Columbia followed closely with ratios around $69 \%$ and $65 \%$. Despite this, the ratio of the number of researchers to $R \& D$ personnel in the business sector in Québec has remained below the national average in recent years. For example, in 2005 this ratio was about 54\% against $60 \%$ for Canada as a whole, $62 \%$ for Prince Edward Island and Alberta, 63\% for Ontario, and British Columbia topped the list with $68 \%$.

One conclusion that emerges from these statistics is that, despite the fact that for nearly three decades Québec businesses have received the most advantageous tax assistance program in Canada for jobs related to R\&D, the proportion of researchers has so far failed to increase significantly compared to the national average. In a recent report, an expert panel on Research and Development charged by the Government of Canada to examine how to strengthen the impact of federal investments in support of a more innovative economy (Innovation Canada: A Call to Action, Canada (2011)) made the recommendation to simplify the Scientific Research and Experimental Development program by basing the tax credit for SMEs on labour-related costs. Clearly, our findings in Québec do not support such a recommendation.

Research question 2: Are R\&D tax incentives (indirect incentives) effective in Québec?

The interest of this question is obvious in a context where government incentives for R\&D from both the Government of Québec and the federal government are heavily weighted towards tax incentives and are among the most generous in the world. Furthermore, these tax incentives are level-based and none of the actual tax incentives are incremental. In 1999, the Government of Québec implemented a tax credit based on the increase in R\&D expenditures as part of an effort to increase the ratio of R\&D expenditures to GDP to the average performance of G7 countries, but only for a five-year period. Thus, this tax credit ended in 2004. In this respect, our mission was to shed light on the question of whether the money spent by the government in R\&D tax incentives gives plenty of "bang for the buck" (BFTB) or, in other words, how much private R\&D increased per dollar of R\&D tax receipts foregone? (Chapter 5). More formally, the BFTB may be thought of as the ratio of cumulative additional $R \& D$ to cumulative government expenses to support $R \& D$.

We estimated the price elasticity of R\&D and found values of -0.10 in the short run and -0.14 in the long run, with a slightly higher elasticity for small firms than for large firms. Using these short and long-run estimated price elasticities, we computed the impacts of increases in provincial tax incentives on the BFTB along 
the entire trajectories towards their new steady states to the saving in government costs to support R\&D along these trajectories.

First, if the level-based provincial tax incentives are increased by $10 \%$, we showed that the BFTB for small firms starts at a value much higher than one, and slowly drops to a value that stays above 1 even after 20 years. Hence for small firms it is beneficial to support R\&D through tax incentives. More R\&D gets created than it costs the government to support it, even after 20 years. For large firms, however, the BFTB starts at 3, but falls below 1. Already after seven years the cumulative additional R\&D no longer covers the cumulative government costs to support this R\&D. By considering all the firms, the BFTB falls below the bar of one after 13 periods. Thus considering the whole adjustment towards a new steady state, the level-based R\&D tax incentives do not come out as very effective. The reason for this ineffectiveness is that level-based tax incentives contain a deadweight loss, namely the tax support on the R\&D that would be done in the absence of all support.

Second, if only the incremental based R\&D tax credit is increased by $10 \%$, there can also be a deadweight loss, but this deadweight loss is likely to be small. By simulating the effect of the incremental tax change on R\&D and government support for R\&D over a period of 20 years, we find that the bang for the buck is about $\mathbf{2 . 9 8}$ for small firms and $\mathbf{2 . 7 9}$ for large firms. The incremental R\&D tax credit is much more effective than the level based tax credit, and its effectiveness does not vary very much by firm size.

Research question 3: Are level-based R\&D tax incentives more efficient than incremental tax incentives?

As emphasized in the preceding research question, the level-based R\&D tax incentives do not come out as very effective because they contain a deadweight loss attributable to the tax support on the R\&D that would be done in the absence of all support. As indicated, this deadweight loss is particularly acute for large firms. For small firms it is not sizeable enough to overcome the R\&D additionality in the short run. Incremental R\&D tax credits do not suffer from this deadweight loss and are from that perspective preferable to level-based tax incentives (Chapter 5).

Research question 4: Is private R\&D spending sensitive to increments in fiscal incentives?

This research question is answered in Chapter 7, where we study the case of Québec firms that were offered a tax benefit of up to three times the value of the existing tax benefit through the government's superdeductions program. More specifically, the question asked in this chapter is whether the reduction of the 
marginal cost of R\&D led by the superdeductions program in Québec prompted companies already benefiting from advantages from the SR\&ED tax credit to increase their R\&D effort. The results obtained show no evidence that such companies increase their $R \& D$ investments when tax assistance is significantly incremented. Thus, there is a certain threshold beyond which government funding becomes less effective. Accordingly, the phenomenon of crowding-out cannot be totally excluded in the case of the Québec superdeductions program, at least in the short run, and this is mainly because the tax assistance is level-based. As argued in the preceding research question, the incremental-based fiscal measures are preferable since they are less sensitive to this problem.

Research question 5: Do public subsidies (direct incentives) increase private R\&D investment? How do these incentives impact firms' productivity?

After assessing the impacts of the indirect measures on private $R \& D$, we naturally looked at the impacts of direct measures. The specificity in the case of Québec is that, since all the firms are de facto offered the possibility to claim tax credits for their R\&D expenditures, subsidies appear as complementary incentives to fiscal incentives. In this framework, an interesting avenue of research was to empirically analyse the impacts of public R\&D subsidies on private $R \& D$ investments and on the productivity growth of firms in a context where fiscal incentives are present.

Our results (Chapter 6 ) show that firms that use public grants for $R \& D$ in conjunction with tax credits for $R \& D$ perform better in terms of $R \& D$ input additionality and in terms of growth productivity than firms that use only tax credits for R\&D. In terms of input additionality, for manufacturing firms in Québec already benefiting from tax credits in the period 1998 to 2004, the average treatment effect of subsidies on R\&D intensity varied between $2 \%$ to $11 \%$. This result was also mirrored in that of low-medium technology firms, but not that of high technology firms. In the latter case, our estimates were a bit higher than the ones in the two previous cases, i.e. they varied between $4 \%$ and $13 \%$. These results suggest the exclusion of possible crowding-out effects in a context where firms are given the choice of adding R\&D grants to R\&D tax credits. Furthermore, the results in the case of high technology firms suggest that in a fiscal environment such as that of Québec, high technology firms may be more responsive than other firms to direct public subsidies.

Regarding the productivity growth, we find positive rates of return to $R \& D$ expenditures funded by public grants and to R\&D expenditures funded privately and by tax credits. More specifically, for each additional dollar of public R\&D grant, output increases by 0.134 dollars in the former case, and for each additional dollar of the remaining R\&D expenditures (funded privately and by tax credits), output increases by 0.322 dollars. It is noteworthy that, although these positive results 
support direct subsidy programs, they also show that the additional return of direct subsidies is positive, but lower than the return on the R\&D financed by private funds or R\&D tax credits. The ratio of both rates of return is of 0.42 , which indicates that public R\&D grants represent $42 \%$ of the productivity induced by both private funds and R\&D tax credits. Finally, from estimation results, the hypothesis that firms in Québec are facing constant return to scale was rejected in favor of decreasing return to scale.

Research question 6: Are the combinations of public intervention methods currently used in Québec to encourage R\&D optimal?

The question of choosing the optimal mix between direct and indirect measures is a central issue in the success of a policy favouring R\&D and innovation. However, there is no clear framework for this purpose. Thus, as we have shown in Section 2.3, the choice of combination differs from country to country. From our analysis, Canada appears as an outlier in comparison to other countries. This also applies to Québec since it is a major R\&D funder in Canada. While most countries use exclusively direct incentives or a mix of incentives weighted toward direct incentives, Canada is the country whose share of funds spent on indirect measures over direct measures is highest: in 2007, the amounts spent on indirect measures represented almost 10 times that of direct measures.

The results obtained in the previous chapters do not suggest that combinations of public intervention methods currently used in Québec to encourage R\&D are optimal. First, the research question 5 indicated that firms that use direct incentives in conjunction with indirect incentives such as tax credits perform better in terms of $R \& D$ input additionality and in terms of growth productivity than firms that use only tax credits for R\&D. Secondly, we also showed that although overall they give positive results, the indirect incentives in the case studied, which are level-based, may result in some cases in deadweight loss or in crowding out.

Research question 7: What is the impact of overall public incentives (direct and indirect) on firms' $R \& D$, innovation, and productivity in Québec? How do firms in Québec compare to firms in other jurisdictions?

Concerning the impact of overall public direct and indirect incentives on firms' R\&D, innovation, and productivity in Québec, the estimation results in Chapter 8 are very conclusive and, as we used the actual amounts of government support received by firms, they therefore strongly add to the evidence on the theoretical determinants concerning the intensity of government support, factors influencing firms' decisions to be innovative, and factors influencing the innovation process and productivity. Among these estimation results, we can highlight the following findings. 
First, an increase of $1 \%$ in government support is associated with a $0.32 \%$ higher probability of being innovative and an increase in innovation expenditures of $0.99 \%$, thus supporting government intervention. In addition, estimation results report high elasticity of product innovation sales with respect to innovation expenditures of 0.83 for sponsored firms. Also, this elasticity is higher for the group of high and medium-high technology firms than for the group of medium-low and low technology firms. Using the results from estimation parameters, a total effect of government support on innovation sales of 0.824 was computed. This means that raising government support by $1 \%$ increases innovation sales by $0.82 \%$. The result above led to the conclusion that sponsored firms perform better than nonsponsored firms.

Concerning the effect of new or significantly improved production processes however, estimation results yielded that there is no evidence, in the case of innovative firms receiving government support in the manufacturing sector in Québec, that the introduction of production process innovation positively affects innovation sales. In other words, we cannot conclude that recipient firms that introduce both product and process innovations have greater returns than those introducing only product innovation. This intriguing result calls for further research.

As regards to the link between innovation and labour productivity, results show that a $1 \%$ increase in firms' innovation sales per employee is associated with a productivity increase of $0.37 \%$ and we found that high and medium-high technology firms are more responsive to government incentives since for this group the elasticity (0.46) is more than twice as large as for the group of medium-low and low technology firms (0.22). The computed total effect of government support on the productivity of subsidized firms is 0.307 . Hence, raising government support by $1 \%$ causes a $0.31 \%$ increase in labour productivity. Finally, we introduced in our estimation approach some of the competition variables being tested recently in the literature, such as the concentration ratio and the global technology frontier, and found that recipient firms in more concentrated industries or closer to the technology frontier spend more on innovation and earn more sales from innovation than those further away, and this is especially the case for high and medium-high technology firms.

Second, in addition to the estimation that led to the results summarized above, we ran another set of experiments in which Québec's manufacturing firms were compared to those of 18 OECD and non-OECD countries using a model developed by an OECD team as part of a microdata project. In this case, the estimation results enabled us to address the following four questions: Which firms are more likely to be innovative? Which firms invest more on innovation? Does spending in innovation translate into sales from product innovation? What is the link between innovation and productivity? We found an elasticity of product innovation sales to innovation expenditures of 0.35 , which falls in the $14-40 \%$ range estimated by the OECD team. 
Lastly, with regards to productivity, we found that a $1 \%$ increase in product innovation sales per employee is associated with an estimated $0.43 \%$ increase in labour productivity in Québec. This is about the same rate as that found for Canada in the OECD study. Hence, we concluded that manufacturing firms in Québec that invest in innovation have a performance in respect of labour productivity comparable to that of OECD countries.

Research question 8: Can we attribute the causes of the lag experienced by Québec in its labour productivity to the underperformance of firms in innovation?

This research question is addressed in Chapter 3 and Chapter 8 as a contribution to a puzzling question: what causes the lag experienced by Québec in its labour productivity compared to other Canadian provinces and major industrialized countries? Indeed, we showed that the R\&D investments of Québec companies have experienced strong growth over the last thirty years, enabling them to reach and surpass the Canadian average and several OECD countries. However, despite this convergence, aggregate productivity continues to lag behind the latter and even behind its immediate neighbor, Ontario, whose growth rate of investments in R\&D firms was less significant during the same period.

On the basis of the results of international comparisons mentioned in the preceding research questions, we can at least claim that innovative firms in the manufacturing sector in Québec have a performance in respect to labour productivity comparable to that of OECD countries and, hence, rule out the hypothesis of under-performance for this group of firms.

At the aggregate level, however, such an assertion cannot be made and we have proposed some hypotheses to explain this productivity lag, but further research is needed to validate them.

\subsection{Policy Implications}

In light of the results found in this research project, we are in position to make some suggestions and recommendations to decision makers. First, the lessons learned from research question 2 about the effectiveness of R\&D tax incentives suggest the need to redefine the measures currently in place. Thus, as emphasized, if the Government of Québec wants to run an efficient fiscal policy in support of R\&D, it would be advised to continue using both the incremental R\&D tax credit and the level-based tax credits in favour of small firms. For large firms, however, level-based tax credits have a serious drawback.

Second, regarding direct support measures, an important implication of the findings in Chapter 6 is that the high tax incentives, low subsidies approach used by 
both the governments of Québec and Canada could be causing a loss of efficiency of the public support system for innovation activities. Indeed, by examining the additionality of public support and productivity, we found that, on average, firms that combine subsidies to tax incentives perform better than firms that use only tax incentives. This leads us to suggest that the governments of Québec and Canada should use more direct incentives as additional policy instrument to tax credits. Moreover, this suggestion is in line with the approach taken by several other countries as it has been demonstrated in this research project. It is also in line with the recommendations' of the Expert Panel on Research and Development (Innovation Canada: A Call to Action, Industry Canada (2011)). From this point on, however, it is more a question of the capacity of public agencies responsible of the attribution of R\&D grants to identify the projects for which R\&D tax credits fail to offer an incentive for the private projects and to find a suitable level of additional funding in a context where firms already benefit from fiscal incentives.

Third, regarding the productivity gap of Québec, we recommend that if the Government of Québec wants to increase firms' labour productivity, it will need to adopt a more active and specific policy for R\&D support in order to more efficienttly target industries or sectors where government intervention is needed.

Fourth, based on the subsidiary issues highlighted in our review of the policy for $R \& D$ and innovation, we can make some recommendations regarding its effectiveness. On the one hand, as often discussed in the literature, innovation policy cannot be discussed separately from the policy on R\&D. To this end, some countries have begun to adopt a more integrated approach in this regard. Québec politics should follow this model. In addition, we believe it would be advantageous to establish an integrated federal-provincial government action for all innovation activities to avoid duplication of support measures, to achieve economies of scale, and especially to increase system efficiency by increasing its ability to identify areas where public intervention is needed. On the other hand, it is important for an efficient policy that the government ensure that public assistance programs be simpler and easier to access. We have shown in Chapter 2 the complexity of the institutional environment for the administration of government programs offered to Québec businesses. Access to some government programs can lead to significant administrative costs such as hiring or the allocation of full-time staff to prepare the documents necessary to access public funding. Companies are sometimes forced to turn to the services of consultants. On this last point, indeed, there is an important industry in Québec that offers consulting services to companies to take over for them and prepare the documentation required to qualify for government assistance. The amounts paid by companies to consulting agencies are not well known, but according to some estimates they are significant. These costs reduce the value of the financial support. 


\subsection{Limitations and Suggestions for Future Research}

This work is an important first step because it helps fill the gap in the literature related to public policies concerning R\&D and innovation in Québec, but it still leaves several questions unanswered. This project would highly benefit from the data by subsidy programs if it were available. Indeed, the present study was conducted using the amount of subsidies received by firms, without distinguishing among subsidy programs. It would be important to continue the detailed analysis by program.

Among the avenues of future research that would complement this study, an important consideration is the social rate of return on tax-supported R\&D. It would be interesting in future work to compare the social returns on government supported R\&D via tax credits and direct subsidies, on government supported and non-supported R\&D, and on R\&D support for large and small firms. Other aspects of policy which were not analyzed in this research project concern the evaluation of the effectiveness of subsidies to research performed in public labs and universities, where costs for the government are high, as demonstrated in Chapter 3. Finally, a study of interest for the purposes of policies with respect to the productivity gap would be to establish a detailed portrait of firms' characteristics in different industries or sectors with respect to their patterns of investment in innovation. 


\section{References}

AliC, J. A., D. C. Mowery, and E. S. Rubin (2003): "U.S. Technology and Innovation Policies: Lessons for Climate Change," Arlington, VA: Pew Center on Global Climate Change.

Almus, M., and D. CZARnitzki (2003): "The Effects of Public R\&D Subsidies on Firms' Innovation Activities: The Case of Eastern Germany," Journal of Business and Economic Statistics, 21, 226-36.

ARROW, K. (1962): "Economic Welfare and the Allocation of Resources for Invention," in The Rate and Direction of Inventive Activity: Princeton University Press, 609-626.

BaghanA, R., and P. Mohnen (2009): "Effectiveness of R\&D Tax Incentives in Small and Large Enterprises in Québec," Small Business Economics, 33, 91-107.

BaghanA, R. S. (2008): "Une évaluation de l'impact du programme québécois de superdéductions à la R-D," in Compendium d'indicateurs de l'activité scientifique et technologique au Québec. Édition 2008, ed. by Institut de la Statistique du Québec, Québec.

BaghanA, R. S. (2010): "Public R\&D Subsidies and Productivity: Evidence from FirmLevel Data in Quebec," United Nations University, Maastricht Economic and social Research and training centre on Innovation and Technology.

Balcerowicz, E., M. Peczkowski, and A. Wziatek-Kubiak (2009): "The Innovation Patterns of Firms in Low and High Technology Manufacturing Sectors in the New Member States," CASE-Center for Social and Economic Research.

BÉLANGER, G. (1980): "La productivité croît-elle trop rapidement Au Québec?," Montréal.

Berger, M. (2009): "The Innovation-Productivity Link: Comparing Thailand with a Sample of OECD Countries," Hong Kong.

BernsteIn, I. J. (1986): "The Effect of Direct and Indirect Tax Incentives on Canadian Industrial R\&D Expenditures," Canadian Public Policy, 12, 438-448.

BÉRUbÉ, C., and P. Mohnen (2009): "Are Firms That Receive R\&D Subsidies More Innovative," Canadian Journal of Economics, 42, 206-225.

Bloom, N., R. Griffith, and J. Van Reenen (2002): "Do R\&D Credits Work? Evidence from a Panel of Countries 1979-97," Journal of Public Economics, 85, 1-31. 
BLUNDELL, R., and M. CostA DiAs (2000): "Evaluation Methods for Non-Experimental Data," Fiscal Studies, 21, 427-468.

BOYER, M. (2001): "La performance économique du Québec: constats et défis," Montreal: Centre universitaire de recherche en analyse des organisations.

Brouwer, E., P. Den Hertog, T. Poot, and J. Segers (2002): " Wbso Nader Beschouwd. Onderzoek Naar De Effectiviteit Van De Wbso," Opdracht van het Ministerie van Economische Zaken.

BUREAU OF LABOR STATISTICS (1989): "The Impact of Research and Development on Productivity Growth," Washington, DC: Bureau of Labor Statistics.

CAPpelen, Å., A. RAKNerud, and M. Rybalka (2008): "The Effects of R\&D Tax Credits on Patenting and Innovations," Oslo: Research Department of Statistics Norway.

Chirinko, R. S., S. M. Fazzari, and A. P. Meyer (1999): "How Responsive Is Business Capital Formation to Its User Cost?: An Exploration with Micro Data," Journal of Public Economics, 74, 53-80.

Cleveland, W. S. (1979): "Robust Locally Weighted Regression and Smoothing Scatterplots," Journal of the American Statistical Association, 74, 859-836.

Cochran, W. G., and D. B. RuBIN (1973): "Controlling Bias in Observational Studies: A Review " Sankhyā: The Indian Journal of Statistics, 35, 417-446.

Corchuelo Martínez-AzúA, B., and E. Martínez-Ros (2008): "Aplicación de los incentivos fiscales a la inversión en I+D en las empresas españolas," Hacienda Pública Española/Revista de Economía Pública, 187, 9-39.

Cornet, M., and B. VRoomen (2005): Hoe Effectief Is Extra Stimulering Van Speur- En Ontwikkelingswerk? : Centraal Planbureau.

CPP (2010): "Productivity and Prosperity in Quebec: 2010 Overview," Montréal: HEC Montréal Centre for Productivity and Prosperity.

CRÉPON, B., E. Duguet, and J. MAIRESSE (1998): "Research, Innovation and Productivity : An Econometric Analysis at the Firm Level," Economics of Innovation and New Technology, 7, 115-158.

CST (2002): Conseil de la science et de la technologie: 30 ans d'histoire. Québec: CST.

CST (2010): "Le financement de l'innovation dans les entreprises," Québec: Conseil de la science et de technologie.

CZARNITZKI, D., P. HANEL, and J. M. RosA (2005): "Evaluating the Impact of R\&D Tax Credits on Innovation: A Microeconometric Study on Canadian Firms," Mannheim: Centre for European Economic Research (ZEW). 
CZARNITZKI, D., and K. Hussinger (2004): "The Link between R\&D Subsidies, R\&D Spending and Technological Performance."

CZARNITZKI, D., and G. LICHT (2006): "Additionality of Public R\&D Grants in a Transition Economy: The Case of Eastern Germany," Economics of Transition, 14, 10131.

Dagenais, M., P. Mohnen, and P. Therrien (2004): "Les firmes canadiennes repondent-elles aux incitations fiscales a la recherche-developpement?," L'Actualite économique/Revue d'analyse économique, 80, 175-205.

DAHLBY, B. (2005): "A Framework for Evaluating Provincial R\&D Tax Subsidies," Canadian Public Policy, 31, 45-58.

DAVENPORT, P. (1981): "L'économie québécoise et le ralentissement de la productivité," Montreal: McGill University.

David, P. A., B. H. HAll, and A. A. Toole (2000): "Is Public R\&D a Complement or Substitute for Private R\&D? A Review of the Econometric Evidence," Research Policy, 29, 497-529.

DAVIDSON, R., and J. G. MACKINNON (1993): Estimation and Inference in Econometrics. Oxford University Press.

De JONG, J. P. J., and W. H. J. Verhoeven (2007): "Evaluatierapport Wbso 2001-2005: Effecten, Doelgroepbereik En Uitvoering," EIM, UNU-MERIT.

DeheJIA, R. H., and S. WAHBA (2002): "Propensity Score-Matching Methods for Nonexperimental Causal Studies," The Review of Economics and Statistics, 84, 151-161.

Department of Finance, Canada (2010a): "Tax Expenditures and Evaluations 2010," Government of Canada, 66.

Department of FinANCE, CANAdA (2010b): "Tax Expenditures: Notes to the Estimates/Projections 2010," Government of Canada.

Department of Finance, Canada, and Revenue Canada (1997): "The Federal System of Income Tax Incentives for Scientific Research and Experimental Development-Evaluation Report," Government of Canada.

Department of Finance Canada (2007): "Tax Expenditures and Evaluations 2006," Government of Canada.

Dinges, M., M. Berger, R. Frietsch, and A. Kaloudis (2007): "Public Versus Private Funded Business R\&D: Sector Specific Specialisation Indices as a Tool for Policy Analysis," Institute of Technology and Regional Policy.

DUGUET, E. (2008): "L'effet du crédit d'impôt recherche sur le financement privé de la recherche: une évaluation économétrique," Évry, France: Centre d'études des politiques économiques de l'université d'évry. 
FAN, J. (1992): "Design Adaptive Nonparametric Regression," Journal of the American Statistical Association, 87, 998-1004.

Fazzari, S. M., G. R. Hubbard, and B. C. Petersen (1988): "Financing Constraints and Corporate Investment," Brookings Papers on Economic Activity, 1, 141-195.

FoRTIN, P. (2001): "Has Quebec's Standard of Living Been Catching Up?," in The State of Economics in Canada: Festschrift in Honour of David Slater: Centre for the Study of Living Standards, 381-402.

GarciA, A., and P. Mohnen (2010): "Impact of Government Support on R\&D and Innovation," Maastricht: UNI-MERIT.

González, X., J. Jaumandreu, and C. PAZó (2005): "Barriers to Innovation and Subsidy Effectiveness," Rand Journal of Economics, 36, 930-950.

GouVERnEMENT DU QUÉBEC (1988): "La maîtrise de notre avenir technologique : un défi à relever, plan d'action québec 1988-1992, Document de consultation," Gouvernement du Québec, 107.

Gouvernement du QuÉBec (2001): "Politique québécoise de la science et de l'innovation : savoir changer le monde," Gouvernement du Québec, 169.

GOUVERNEMENT DU QUÉBEC (2004): Briller parmi les meilleurs : la vision et les priorités du gouvernement du Québec. Québec: Gouvernement du Québec.

Green, A. G. (1971): Regional Aspects of Canada's Economic Growth. Toronto: University of Toronto Press.

Greene, W. (2003): Econometric Analysis. New Jersey: Prentice Hall.

Griffith, R., E. Huergo, J. Mairesse, and B. Peters (2006): "Innovation and Productivity across Four European Countries," Oxford Review of Economic Policy, 22, 483-498.

GRILICHES, Z. (1973): "Research Expenditures and Growth Accounting," in Science and Technology in Economic Growth, ed. by B.R. Williams. London: Macmillan, 59-83.

GRILICHES, Z., and J. MAIRESSE (1991): "R\&D and Productivity Growth: Comparing Japanese and U.S. Manufacturing Firms," National Bureau of Economic Research, Inc.

Guellec, D., and B. Van Pottelsberghe (2000): "The Impact of Public R\&D Expenditure on Business R\&D," Paris: OECD, Directorate for Science, Technology and Industry.

HÆEGELAND, T., and J. MøEN (2007): "Input Additionality in the Norwegian R\&D Tax Credit Scheme," Statistics Norway. 
Hall, B., F. LotTI, and J. MaIResSe (2009): "Innovation and Productivity in Smes: Empirical Evidence for Italy," Small Business Economics, 33, 13-33.

HaLL, B., and J. Van ReEnen (2000): "How Effective Are Fiscal Incentives for R\&D? A Review of the Evidence," Research Policy, 29, 449-469.

HALL, B. H. (1993): "R\&D Tax Policy During the Eighties: Success or Failure?," Tax Policy and the Economy, 7, 1-36.

HALL, B. H., and J. MAIRESSE (1995): "Exploring the Relationship between R\&D and Productivity in French Manufacturing Firms," Journal of Econometrics, 65, 263-293.

HALPERN, L., and B. MuRAKÖZY (2010): "R\&D Subsidies and Firm Performance in Hungary," MICRO-DYN.

HeCKMAN, J., H. ICHIMURA, J. SMITH, and P. TOdD (1998a): "Characterizing Selection Bias Using Experimental Data."

HeCKMAN, J. J. (1979): "Sample Selection Bias as a Specification Error," Econometrica, $47,153-161$.

HeCKMAN, J. J., H. ICHIMURA, and P. TOdD (1998b): "Matching as an Econometric Evaluation Estimator," Review of Economic Studies, 65, 261-94.

HeCKMAN, J. J., H. ICHIMURA, and P. E. Todd (1997): "Matching as an Econometric Evaluation Estimator: Evidence from Evaluating a Job Training Programme," Review of Economic Studies, 64, 605-54.

HeCKMAN, J. J., and R. RoBb, JR. (1985): "Alternative Methods for Evaluating the Impact of Interventions : An Overview," Journal of Econometrics, 30, 239267.

INDUSTRY CANADA (2011): "Innovation Canada: A Call to Action," Gouvernement du Canada.

Jaffe, A. B., M. Trajtenberg, and R. Henderson (1993): "Geographic Localization of Knowledge Spillovers as Evidenced by Patent Citations," The Quarterly Journal of Economics, 108, 577-98.

Johansson, B., and H. LöÖF (2009): "Innovation, R\&D and Productivity - Assessing Alternative Specifications of Cdm-Models," Royal Institute of Technology, CESIS - Centre of Excellence for Science and Innovation Studies.

KIRNeR, E., S. KINKEL, and A. JAEger (2009): "Innovation Paths and the Innovation Performance of Low-Technology Firms-an Empirical Analysis of German Industry," Research Policy, 38, 447-458.

Kleibergen, F., and R. PaAp (2006): "Generalized Reduced Rank Tests Using the Singular Value Decomposition," Journal of Econometrics, 133, 97-126. 
KLETTE, T. J., and Z. GRILICHES (1996): "The Inconsistency of Common Scale Estimators When Output Prices Are Unobserved and Endogenous," Journal of Applied Econometrics, 11, 343-61.

Klette, T. J., J. Moen, and Z. Griliches (2000): "Do Subsidies to Commercial R\&D Reduce Market Failures? Microeconometric Evaluation Studies," Research Policy, 29, 471-95.

LACH, S. (2002): "Do R\&D Subsidies Stimulate or Displace Private R\&D? Evidence from Israel," Journal of Industrial Economics, 50, 369-90.

LANDRY, R., and N. AMARA (2003): "Effects of Sources of Information on Novelty of Innovation in Canadian Manufacturing Firms," in Understanding Innovation in Canadian Industry, ed. by Fred Gault. Montreal, Kingston: McGillQueen's University Press.

LebeAu, D. (1996): "Les mesures d'aide fiscale à la R-D et les entreprises québécoises," in L'efficacite des mesures d'aide fiscale à la $R-D$ des entreprises du Canada et du Québec, ed. by Conseil de la science et de la technologie du Québec. Sainte-Foy, Québec: Gouvernement du Québec.

LeE, F., and K. NeWton (2001): "Innovation by Smes in the Kbe," Journal of Small Business and Entrepreneurship, Spring.

Leuven, E., and B. SiAnesI (2003): "Psmatch2: Stata Module to Perform Full Mahalanobis and Propensity Score Matching, Common Support Graphing, and Covariate Imbalance Testing," Boston College Department of Economics.

LI, Q., and J. S. RACINE (2007): Nonparametric Econometrics: Theory and Practice. Princeton University Press.

LOKSHIN, B., and P. MoHNEN (2007): "Measuring the Effectiveness of R\&D Tax Credits in the Netherlands," United Nations University, Maastricht Economic and social Research and training centre on Innovation and Technology.

LöÖF, H., and A. Heshmatı (2006): "On the Relation between Innovation and Performance: A Sensitivity Analysis," Economics of Innovation and New Technology, 15, 317-344.

LöÖF, H., A. HeshmatI, R. AsPlund, and A. NAAS (2001): "Innovation and Performance in Manufacturing Industries: A Comparison of the Nordic Countries," SSE and EFI Working Paper NO.457.

MAdDALA, G. S. (1983): Limited Dependent and Qualitative Variables in Econometrics Cambridge, England Cambridge University Press.

MAdore, O. (1989): "Scientific Research and Experimental Development: Tax Policy," Ottawa: Parliament of Canada. 
MAIRESSE, J., and B. MuLKAY (2004): "Une évaluation du crédit d'impôt recherche en France, 1980-1997," Revue d'économie politique, 114, 747-778.

MAnsfield, E., and L. SWitzer (1985a): "The Effects of R\&D Tax Credits and Allowances in Canada," Research Policy, 14, 97-107.

MAnsfield, E., and L. Switzer (1985b): "How Effective Are Canada's Direct Tax Incentives for R and D?," Canadian Public Policy, 11, 241-246.

MAté-Garcia, J. J., and J. M. RodRIGUeZ-Fernandez (2008): "Productivity and R\&Amp;D: An Econometric Evidence from Spanish Firm-Level Data," Applied Economics, 40, 1827 - 1837.

McFetRIDGE, D. G., and J. P. WARDA (1983): Canadian R\&D Incentives: Their Adequacy and Impact. Toronto, Canada: Canadian Tax Foundation.

MIGUÉ, J.-L. (1998): Étatisme et déclin du Québec: Bilan de la révolution tranquille. Montreal: Varia.

Ministère des FinANCES dU QuÉBec (2003a): "Additional Information on the Fiscal Measures," Gouvernement du Québec.

Ministère des FinANCES DU QuÉBEC (2003b): "Tax Expenditures 2003," Gouvernement du Québec.

MinistÈRE des FinANCES du QuÉBEC (2005): "Tax Expenditures 2005," Gouvernement du Québec.

Ministère des FinANCES du QuÉBEC (2008): "Tax Expenditures 2008," Gouvernement du Québec.

Ministère des FinANCES du QuÉBEC (2009): "Tax Expenditures 2009," Gouvernement du Québec.

MinistèRE des FinANCES DU QuÉBEC (2010): "Tax Expenditures 2010," Gouvernement du Québec.

Ministère du DÉVELOPPEMENT ÉCONOMIQUe, DE L'INNOVATION ET DE L'EXPORTATION, QuÉBeC (2006): "Stratégie québécoise de la recherche et de l'innovation: un Québec innovant et prospère," Gouvernement du Québec, 80.

Ministère du DÉVEloppement ÉCONOMIQUe, DE L'INNOVATION ET DE L'EXPORTATION, QUÉBEC (2010): "Stratégie québécoise de la recherche et de l'innovation 20102013: mobiliser innover prospérer," Québec: Gouvernement du Québec, 45.

Ministère du DéVELopPement ÉCONOMIQUe, de L'INNOVATION ET DE L'EXPORTATION, QuÉBEC (2005): L'avantage québécois. Québec: Gouvernement du Québec. 
Mohnen, P. (2000): "R\&D Tax Incentives: Issues and Evidence," in The Political Economy of Industrial Policy in Europe, the Member States, ed. by Damien J. Neven, and L. H. Rölle. Bohn (Berlin): Sigma Rainer, 183-199.

NeLSON, R., R. (1959): "The Simple Economics of Basic Scientific Research," Journal of Political Economy, 67, 297-306.

OECD (1996): The Measurement of Scientific and Technological Activities: Proposed Guidelines for Collecting and Interpreting Technological Data (Oslo Manual). OECD.

OECD (1997): The Measurement of Scientific and Technological Activities: Proposed Guidelines for Collecting and Interpreting Technological Data (Oslo Manual). OECD.

OECD (1998): Technology, Productivity and Job Creation: Best Policy Practice. OECD Publishing.

OECD (2002): Frascati Manual 2002: Proposed Standard Practice for Surveys on Research and Experimental Development. OECD.

OECD (2005): The Measurement of Scientific and Technological Activities: Guidelines for Collecting and Interpreting Innovation Data (Oslo Manual). OECD.

OECD (2009): Innovation in Firms: A Microeconomic Perspective. OECD.

OECD (2010): Measuring Innovation: A New Perspective. Paris: OECD.

OECD (2011): "Innovation Union Competitiveness Report 2011," OECD.

PAGÉ, L. (1995): "L'incidence des subventions sur les dépenses privées en recherche et développement: une approche microéconomique," Sherbrooke: Université de Sherbrooke.

PAQUET, G. (1999): "Oublier la révolution tranquille," Montreal: Liber.

Parisi, M. L., F. Schiantarelli, and A. Sembenell (2006): "Productivity, Innovation and R\&D: Micro Evidence for Italy," European Economic Review, 50, 2037-2061.

Parsons, M., and N. PhILlips (2007): "An Evaluation of the Federal Tax Credit for Scientific Research and Experimental Development," Department of Finance, Canada.

Polder, M., G. v. LeeuWen, P. Mohnen, and W. Raymond (2009): "Productivity Effects of Innovation Modes," University Library of Munich, Germany.

ReVenu QuéBec (2008): "Tax Assistance for Scientific Research and Experimental Development," Government of Québec.

Rosenbaum, P. R., and D. B. Rubin (1983): "The Central Role of the Propensity Score in Observational Studies for Causal Effects," Biometrika, 70, 41-55. 
Rosenbaum, P. R., and D. B. Rubin (1985): "Constructing a Control Group Using Multivariate Matched Sampling Methods That Incorporate the Propensity Score," The American statistician, 39, 33-38.

RUBIN, D. B. (1977): "Assignment to Treatment Group on the Basis of a Covariate," Journal of Educational and Behavioral Statistics, 2, 1-26.

SASCHA, O. B., and I. ANDREA (2002): "Estimation of Average Treatment Effects Based on Propensity Scores," Stata Journal, 2, 358-377.

SMITH, J., and P. TODD (2003): "Does Matching Overcome Lalonde's Critique of Nonexperimental Estimators?," University of Western Ontario, CIBC Human Capital and Productivity Project.

SMITH, J., and P. TODD (2005): "Does Matching Overcome Lalonde's Critique of Nonexperimental Estimators?," Journal of Econometrics, 125, 305-353.

Smith, V., M. Dilling-Hansen, T. ERiksson, and E. S. Madsen (2004): "R\&D and Productivity in Danish Firms: Some Empirical Evidence," Applied Economics, $36,1797-1806$.

Statistics CANAda (2002): "Industrial Research and Development (2002 Intentions), Catalogue No : 88-202-Xif," Statistics Canada.

Stock, J. H., and M. Yogo (2005): "Testing for Weak Instruments in Linear Iv Regression," in Identification and Inference for Econometric Models: Essays in Honor of Thomas Rothenberg, ed. by Donald W. K. Andrews, and James H. Stock. Cambridge: Cambridge University Press, 80-108.

StONE, C. J. (1977): "Consistent Nonparametric Regression," Annals of Statistics, 5, 595-620.

TESSIER, S., and D. LAGACÉ (2003): "Productivité et activité en R-D des entreprises manufacturières québécoises : constats et implications," Gestion, 28.

TherRien, P., and P. HAnel (2009): "Innovation and Establishments' Productivity in Canada: Results from the 2005 Survey of Innovation," Centre interuniversitaire de recherche sur la science et la technologie (CIRST).

Therrien, P., and P. Hanel (2011): "Innovation and Productivity in Canadian Manufacturing Establishments," International Productivity Monitor, Fall 2011.

TRÉPANIER, M. (1992): Politique de la science au québec et autonomie du champ scientifique. Société québécoise de science politique.

van Leeuwen, G., and S. Faroogul (2008): "Ict, Innovation and Productivity," in Information Society: Ict Impact Assessment by Linking Data from Different Sources, ed. by Eurostat: Eurostat, chap. 12. 
WAKELIN, K. (2001): "Productivity Growth and R\&D Expenditure in Uk Manufacturing Firms," Research Policy, 30, 1079-1090.

WALLSTEN, S. J. (2000): "The Effects of Government-Industry R\&D Programs on Private R\&D: The Case of the Small Business Innovation Research Program," RAND Journal of Economics, 31, 82-100.

WARDA, J. P. (1999): "Measuring the Attractiveness of R\&D Tax Incentives: Canada and Major Industrial Countries," Ottawa: Statistics Canada.

You, J.-I. (1995): "Small Firms in Economic Theory," Cambridge Journal of Economics, $19,441-462$. 


\section{List of Abbreviations and}

\section{Acronyms}

ACER

ACFAS

ADRQ

AQVIR

ASM

ASML

BDC

BERD

CCE

CCPC

CCTT

CDB

CDTI

CÉROM

CFI

ClHR

CIS 4

CLT

CMM

CNE

CNNTQ
Centre de recherche, de développement et de transfert technologique en acériculture

Association canadienne-française pour l'avancement des sciences

Administrative Data from Revenu Québec

Agence québécoise de valorisation de la industrielle de la recherche

Annual Survey of Manufactures, ancestor of the ASML

Annual Survey of Manufactures and Logging that replaced the ASM

Business Development Bank of Canada

Business expenditure on Research and Development

E-Commerce Place

Canadian-controlled private corporations

Centres collégiaux de transfert de technologie

Biotechnology Development Centres

Information Technology Development Centres

Centre de recherche sur les grains

Canada Foundation for Innovation

Canadian Institutes of Health Research

Fourth Community Innovation Statistics

Centres de liaison et de transfert

Cité Multimédia de Montréal

New Economy Centres

Centre national des nouvelles technologies de Québec 
COREM Consortium de recherche minérale

CPP Centre for Productivity and Prosperity, HEC

CPS Conseil de la Politique Scientifique

CQRS Conseil québécois de la recherche sociale

CRA Canada Revenue Agency

CRIQ Centre de recherche industrielle du Québec

CRSAD Centre de recherche en sciences animales de Deschambault

CSA Canadian Space Agency

CST Conseil de Science et de Technologie

DIP Defence Industry Productivity Program

DIRP Defence Industrial Research Program

DRB Defence Research Board

DWH Durbin-Wu-Hausman augmented regression test

EFTA European Free Trade Association

EU European Union

FAI Fonds de l'autoroute de l'information

FCAC Programme de subvention de formation de chercheurs et d'action concertée

FDT Fonds de développement technologique

Fonds FCAR Fonds pour la formation de chercheurs et l'aide à la recherche

FQRNT Fonds de recherche du Québec - Nature et technologies

FQRSC Fonds de recherche du Québec - Société et culture

FRSQ Fonds pour la recherche en santé du Québec

FTE Full-time equivalent

GDP Gross Domestic Product

GERD Gross domestic expenditure on R\&D

HEC École des hautes études commerciales, Montréal

HSF Health Services Fund

ICT Information and communications technology 
IDB

IGIF

INRS

IRAP

IRDA

IRDIA

IREQ

IRS

IS2005

ISQ

ITC

ITO

LTÉÉ

MAPAQ

MDEIE

MELS

MLE

MRC

MRNF

NABST

NAICS

NCE

NEQ

NESTI

NRC

NSERC

OECD
Industrial Development Bank of Canada

Inspector General of Financial Institutions of Québec

Institut national de la recherche scientifique

Industrial Research Assistance Program

Institut de recherche et de développement en agroenvironnement

Industrial research and development incentives act

Institut de recherche d'Hydro-Québec

Internal Revenue Service

Survey of innovation 2005, Statistics Canada

Institut de la statistique du Québec

Investment tax credit

Industrial Technologies Office

Laboratoire des technologies électrochimiques et des électrotechnologies

Ministère de l'Agriculture, des Pêcheries et de l'Alimentation

Ministère du Développement économique, de I'Innovation et de I'Exportation du Québec

Ministère de l’Éducation, du Loisir et du Sport

Full-information maximum likelihood estimator

Medical Research Council of Canada

Ministère des Ressources naturelles et de la Faune

National Advisory Board on Science and Technology

North American Industry Classification System

Networks of Centres of Excellence

Numéro d'entreprise du Quebéc

National Experts in Science and Technology

National Research Council of Canada

Natural Sciences and Engineering Research Council of Canada

Organisation for Economic Co-operation and Development 
PAI

Programme d'appui à l'innovation

PAIT

Program for Advancement of Industrial Technology

PART Programme d'aide à la recherche technologique

PPP

Purchasing Power Parity

PSIP

Program for Strategic Industrial Projects

PSR

Programme pour le soutien à la recherche (MDEIE)

QR\&E

Qualified Research Expenditure

R\&D

Research and Development

R\&E

Research and experimental expenditure

RDCI

Survey of Research and Development in Canadian Industry

REXFOR

SADI

Société de récupération, d'exploitation et de développement forestiers du Québec

SDI Société de développement industriel du Québec

SME Small and Medium Sized Enterprise

SOQUIP Société québecoise $d$ 'initiative pétrolière

SQRI Stratégie québécoise de la recherche et de l'innovation

SR\&ED Scientific research and experimental development

SSHRC Social Sciences and Humanities Research Council

TPC Technology Partnerships Canada

UES Unified Enterprise Survey program of Statistics Canada

VRQ Valorisation-Recherche Québec 


\section{Summary}


Chapter 1, General Introduction, introduced the topic of the thesis and presented its relevance and the research questions addressed.

For a long time, Québec has offered businesses generous direct and indirect incentives for R\&D and innovation which, in the case of indirect incentives, are among the largest in the world. However, it is not clear whether these government incentives actually reach their objective, that is to say, that they lead to a significant increase in private investment in R\&D capable of driving the growth of innovation and of labour productivity outputs. It is even less clear since, in spite of these generous incentives, Québec continues to lag far behind its neighbors with regards to its productivity. In the econometric literature, there is practically no published work assessing the effectiveness of these government incentives in regard to these issues. This study aimed to fill this gap by empirically assessing the effectiveness of direct and indirect measures of government support to R\&D and innovation on both the private investment in R\&D of firms and their resulting economic performances, in particular in terms of innovation output and labour productivity. To address these issues, specific research questions were formulated on the basis of the available data. The specific research questions, which together form this thesis, were:

1) Does fiscal policy subsidizing salaries and wages of researchers translates into increased ratios of researchers in total employment? (Chapter 3)

2) Are R\&D tax incentives (indirect incentives) effective in Québec? In particular, how much private R\&D increases per dollar of R\&D tax receipts foregone? (Chapter 5)

3) Are level-based R\&D tax incentives more efficient than incremental tax incentives? (Chapter 5)

4) Is private R\&D spending sensitive to increments in fiscal incentives? (Chapter 7)

5) Do public subsidies (direct incentives) increase private R\&D investment? How do these incentives impact firms' productivity? (Chapter 6)

6) Are the combinations of public intervention methods currently used in Québec to encourage R\&D optimal? (Chapter 6)

7) What is the impact of overall public incentives (direct and indirect) on firms' $R \& D$, innovation, and productivity in Québec? How do firms in Québec compare to firms in other jurisdictions? (Chapter 8 )

8) Can we attribute the causes of the lag experienced by Québec in its labour productivity to the underperformance of firms in innovation? (Chapter 3 and Chapter 8) 
Chapter 2, Overview, defined the key concepts such as "research and experimental development" according to legislation applicable in Québec. It also addressed the justification of government support in R\&D and innovation and, finally, it presented the provincial and federal administrative environment for the funding of R\&D and innovation.

Chapter 3, R\&D and Innovation Policies in Québec, provided an overview of where Québec stands with regards to $R \& D$ and innovation. It first presented a historical overview of R\&D and innovation policies in Québec. It then presented an in-depth review of direct and indirect incentives, including their costs, offered to $R \& D$ performers and innovators located in Québec by both the federal government and the Government of Québec. The chapter included an interprovincial comparison of R\&D incentives offered to firms and presented the statistics on R\&D and innovation in Québec, in particular those linked to private and public investments. In addition, it presented an interprovincial and global comparison in order to situate Québec in the world. Finally, it discussed the R\&D intensity and Québec's productivity gap issue in light of the statistics presented.

Chapter 4, Data Sources and Construction of Datasets, described the sources of databases and the construction of the datasets used in the empirical analysis. The approach used and the hurdles encountered in the process of linking the files from Statistics Canada surveys and administrative data from Revenu Québec was addressed. It also presented the results of the process of linking the datafiles.

Chapter 5, Effectiveness of R\&D Tax Incentives in Manufacturing Sector in Québec, dealt with the evaluation of the effectiveness of R\&D tax incentives in Québec, using manufacturing firm data from 1997 to 2003 originating from R\&D surveys, annual surveys of manufactures and administrative data. The estimated price elasticity of R\&D is -0.10 in the short run and -0.14 in the long run, with a slightly higher elasticity for small firms than for large firms. We showed that there is a deadweight loss associated with level-based R\&D tax incentives that is particularly acute for large firms. For small firms it is not sizeable enough to suppress the R\&D additionality, at least not during quite a number of years after the initial tax change. Incremental R\&D tax credits do not suffer from this deadweight loss and are, from that perspective, preferable to level-based tax incentives. From these results, we recommended that if it wants to run an efficient fiscal policy in support of R\&D, the province of Québec should continue using both the incremental R\&D tax credit and the level-based tax credits in favour of small firms. It is precisely small firms that have difficulty in getting outside financing for their R\&D efforts. Lastly, we suggested that it would be interesting in future work to compare the social returns 
on government supported R\&D via tax credits and direct subsidies, on government supported and non-supported $R \& D$, and on $R \& D$ support for large and small firms.

Chapter 6, Public R\&D Subsidies and Productivity: Evidence from Firm-Level Data in Québec, empirically analyzed the impacts of public R\&D grants on private $R \& D$ investments and on the productivity growth of the manufacturing firms within a context where fiscal incentives are present. Using the conditional semiparametric difference-in-differences estimator on longitudinal data from Québec, we showed that firms that use public grants for $R \& D$ in conjunction with tax credits for $R \& D$ perform better in terms of R\&D input additionality than firms that use only tax credits for R\&D. We then used a production function to assess the effectiveness of public R\&D grants in the productivity growth of firms. In particular, we found in the first step of the study that the R\&D intensity of firms in the former group is higher than that of firms in the latter group. In the second step of the study, the first main result was that the rate of return to $R \& D$ expenditures funded by public $R \& D$ grants is of 0.134 i.e. for each additional dollar of public R\&D grant, output increase by 0.134 dollars. The second main result was that public R\&D grants represent $42 \%$ of the productivity induced by both private funds and R\&D tax credits. This ratio supports the use of public R\&D grants as an additional policy instrument to tax credits.

Chapter 7, Is Private R\&D Spending Sensitive to Increments in Fiscal Incentives? Evidence from the Superdeductions Program in Québec, examined the sensitivity of $R \& D$ private spending to increments in fiscal programs for R\&D support. Specifically, it determined whether companies receiving superdeductions have passed the surplus tax gain of this new aid provided over the tax credits on to their R\&D spending. In other words, the main question addressed was whether the reduction of the marginal cost of R\&D led by the superdeductions program in Québec prompted companies already benefiting from a tax advantage to increase their $R \& D$ effort. The results obtained showed no evidence that the firms already receiving some tax assistance increase their R\&D investments when tax assistance is significantly incremented. Thus, there is a certain threshold beyond which government funding becomes less effective. Accordingly, the phenomenon of crowding-out could be totally excluded in the case of Québec superdeductions, at least in the short run, and this is mainly because the tax assistance is level-based.

Chapter 8, Impact of Government Funding on R\&D, Innovation, and Productivity: Results from the Innovation Survey Microdata in Québec, analyzed the impacts of government funding on innovation output and labour productivity using data from manufacturing firms in Québec from 2002 to 2004 on a model in which government incentives may increase the profit from innovating. Building on its 
ability to control for the intensity of government support, selectivity and potential simultaneity bias, the model added to the evidence that sponsored firms perform better than non-sponsored firms. In particular, an increase of $1 \%$ in government support is associated with a $0.32 \%$ higher probability of being innovative, thus supporting government intervention. In addition, estimation results report high elasticity of innovation sales with respect to innovation expenditures of 0.83 for sponsored firms and labour productivity elasticity with regards to firms' innovation sales per employee of 0.37 . With regards to the impact of government funding, results showed that a $1 \%$ increase in government support is associated with an increase in innovation expenditures of $0.99 \%$, that is, almost $1 \%$. On the other hand, raising government support by $1 \%$ increases innovation sales by $0.82 \%$ and firms' labour productivity by $0.31 \%$. In addition, the results of Québec's manufacturing firms were compared to those of 18 OECD and non-OECD countries using a model developed by an OECD team as part of a microdata project. The results showed that manufacturing firms in Québec that invest in innovation have a performance in respect of labour productivity comparable to that of OECD countries. The policy recommendation arising from these results was that, in its effort to reduce the productivity gap, the Government of Québec should adopt more active and specific policies for R\&D and innovation support in order to target more specifically the industries or sectors where government intervention is needed.

Chapter 9, Conclusions, presented the main findings for the specific research questions addressed. It then presented policy implications and recommendations. Lastly, it highlighted the limitations and drawn avenues for future research. 

Summary in Dutch (Samenvatting) 


\section{De impact van overheidsincentives op onderzoek en ontwikkeling (R\&D), innovatie en productiviteit: een micro-econometrische analyse van het geval Québec}

In Hoofdstuk 1, Algemene inleiding, wordt het onderwerp van de dissertatie ingeleid en worden de relevantie ervan en de behandelde onderzoeksvragen voorgesteld.

Québec biedt bedrijven al lange tijd aanzienlijke directe en indirecte incentives aan voor R\&D en innovatie, die in het geval van de indirecte incentives bij de grootste van de wereld horen. Het is echter niet duidelijk of die overheidsincentives hun doel bereiken, namelijk leiden tot een aanzienlijke groei in de private investeringen in R\&D die in staat zijn de groei van de output van de innovatie en de arbeidsproductiviteit te stuwen. Het is zelfs minder duidelijk omdat Québec ondanks die aanzienlijke incentives op het vlak van productiviteit flink achter ligt op zijn buren. In de econometrische literatuur is er bijna geen gepubliceerd werk waarin de doeltreffendheid van die overheidsincentives wordt geanalyseerd met betrekking tot deze thema's. In deze studie wordt ernaar gestreefd dit hiaat op te vullen door de doeltreffendheid empirisch te evalueren van directe en indirecte overheidssteun aan R\&D en innovatie aan zowel de private investeringen in $R \& D$ in bedrijven als aan hun economische prestatie, vooral op het vlak van innovatieoutput en arbeidsproductiviteit. Om deze thema's te behandelen werden er specifieke onderzoeksvragen geformuleerd op grond van de beschikbare gegevens. De specifieke onderzoeksvragen die samen deze dissertatie vormen, zijn:

9) Vertaalt het fiscaal beleid van het subsidiëren van de salarissen en lonen van onderzoekers zich in een stijging van het aantal onderzoekers? (Hoofdstuk 3)

10) Zijn belastingsincentives op het vlak van $R \& D$ (indirecte incentives) in Québec doeltreffend? Hoeveel stijgt de private R\&D per dollar aan belastingsvoordeel in het bijzonder? (Hoofdstuk 5)

11) Zijn op niveaus gebaseerde belastingsincentives voor $R \& D$ doeltreffender dan incrementele belastingsincentives? (Hoofdstuk 5)

12) Zijn de private uitgaven aan R\&D gevoelig aan stijgingen van de fiscale incentives? (Hoofdstuk 7)

13) Vergroten openbare subsidies (directe incentives) de private investeringen in $R \& D$ ? Hoe hebben deze incentives een invloed op de productiviteit van de bedrijven? (Hoofdstuk 6) 
14) Worden er momenteel combinaties van openbare interventiemethoden gebruikt in Québec om R\&D optimaal aan te moedigen? (Hoofdstuk 6)

15) Wat is de impact van algemene openbare incentives (directe en indirecte) op $R \& D$, de innovatie en de productiviteit van bedrijven in Québec? Hoe presteren bedrijven in Québec in vergelijking met bedrijven in andere rechtsgebieden? (Hoofdstuk 8)

16) Kunnen we de oorzaken van de achterstand van Québec op het vlak van zijn arbeidsproductiviteit toeschrijven aan de ondermaatse prestatie van de bedrijven op het vlak van innovatie? (Hoofdstuk 3 en Hoofdstuk 8)

In Hoofdstuk 2, Overzicht, worden de belangrijkste concepten zoals "onderzoek en experimentele ontwikkeling" gedefinieerd in de zin van de toepasbare wetgeving van Québec. In het hoofdstuk wordt ook de rechtvaardiging van de overheidssteun voor R\&D en innovatie behandeld en ten slotte worden de provinciale en federale administratieve omgevingen voor de financiering van R\&D en innovatie voorgesteld.

Hoofdstuk 3, R\&D en Innovatiebeleid in Québec geeft een overzicht van waar Québec staat op het vlak van R\&D en innovatie. Eerst wordt er een historisch overzicht gepresenteerd van het beleid op het vlak van R\&D en innovatie in Québec. Daarna worden er diepere beoordelingen van directe en indirecte incentives voorgelegd, met inbegrip van hun kosten, die door de federale regering en de regering van Québec aangeboden werden aan onderzoekers, ontwikkelaars en vernieuwers. Deze presentatie wordt vervolledigd door een interprovinciaal vergelijk van de R\&D-incentives die aangeboden worden aan bedrijven. Vervolgens worden de statistieken van R\&D en innovatie in Québec voorgesteld, vooral diegene die verbonden zijn aan private en openbare investeringen in R\&D en innovatie. Bovendien wordt er een interprovinciale en globale vergelijking gepresenteerd om Québec te situeren in de wereld. Ten slotte worden de R\&D-intensiteit en de productiviteitskloof in Québec besproken in het licht van de voorgelegde statistieken.

Hoofdstuk 4, Gegevensbronnen en constructie van datasets, beschrijft de bronnen van de databases en de constructie van datasets die gebruikt werden bij de empirische analyse. Het presenteert de gebruikte aanpak en de hindernissen die zich opwierpen bij het proces van het linken van de bestanden van de enquêtes van Statistics Canada en de administratieve gegevens van Revenu Québec. Daarna wordt het proces van het linken van de gegevensbestanden gepresenteerd.

In hoofdstuk 5, Doeltreffendheid van belastingsincentives voor $R \& D$ in de productiesector in Québec, wordt de evaluatie besproken van de doeltreffendheid 
van belastingsincentives voor R\&D in Québec, door middel van gegevens van producerende bedrijven van 1997 tot 2003 afkomstig uit enquêtes over R\&D, jaarlijkse enquêtes van fabrikanten en administratieve gegevens. De geschatte prijselasticiteit van $R \& D$ is $-0,10$ op korte termijn en $-0,14$ op lange termijn, met een licht hogere elasticiteit voor kleine bedrijven dan voor grote bedrijven. We hebben aangetoond dat er een deadweight loss geassocieerd wordt met op niveaus gebaseerde belastingsincentives voor R\&D dat vooral acuut is voor grote bedrijven. Bij kleine bedrijven is het niet groot genoeg om de R\&D-additionaliteit te onderdrukken, tenminste niet gedurende een vrij groot aantal jaren na de oorspronkelijke belastingswijziging. Incrementele R\&D-belastingsvoordelen zijn niet onderhevig aan dit welvaartsverlies en genieten uit dat perspectief de voorkeur tegenover op niveau gebaseerde belastingsincentives. Op grond van die resultaten hebben we aanbevolen dat de provincie Québec, als ze een doeltreffend fiscaal beleid wil voeren ter ondersteuning van $R \& D$, zowel de incrementele R\&Dbelastingsvoordelen als de op niveaus gebaseerde belastingsincentives moet blijven gebruiken ten behoeve van kleine bedrijven. Het zijn net de kleine bedrijven die moeilijkheden hebben om financiering van buitenaf te vinden voor hun inspanningen op het vlak van R\&D. Ten slotte hebben we gesuggereerd dat het bij toekomstig werk interessant zou zijn om het sociaal rendement te vergelijken op door de regering ondersteunde R\&D via belastingsvoordelen en directe subsidies, op door de regering gesteunde en niet-gesteunde R\&D en en op de R\&Dondersteuning voor grote en kleine bedrijven.

Hoofdstuk 6, Openbare subsidies voor R\&D en Bewijs van Productiviteit van gegevens op bedrijfsniveau in Québec, voert een empirische analyse door van de impact van openbare toelagen voor $R \& D$ op private investeringen in $R \& D$ en op de productiviteitsgroei van de producerende bedrijven in een context waar er fiscale incentives aanwezig zijn. Door de conditionele semiparametrische difference-indifferences-schatter te gebruiken op longitudinale gegevens van Québec hebben we getoond dat bedrijven die openbare toelagen gebruiken voor $R \& D$ in combinatie met belastingsvoordelen voor R\&D beter presteren op het vlak van de additionele $R \& D$-input dan bedrijven die alleen belastingsvoordelen voor R\&D gebruiken. We hebben vervolgens een productiefunctie gebruikt om de doeltreffendheid te evalueren van openbare R\&D-toelagen in de productiviteitsgroei van bedrijven. In het bijzonder hebben we in de eerste stap van de studie opgemerkt dat de R\&Dintensiteit van bedrijven in de eerste groep hoger is dan die van bedrijven in de laatste groep. In de tweede stap van de studie is het eerste voornaamste resultaat dat de rendementsratio op uitgaven voor $R \& D$ die gefinancierd worden door openbare R\&D-toelagen 0,134 is. Dus groeit de output met 0,134 dollar voor elke extra dollar openbare R\&D-toelage. Het tweede voornaamste resultaat is dat openbare R\&D-toelagen $42 \%$ van de productiviteit vormen die veroorzaakt wordt 
door openbare toelagen en belastingsvoordelen voor R\&D. Deze ratio ondersteunt het gebruik van openbare toelagen voor $R \& D$ als een beleidsinstrument ter aanvulling van belastingsvoordelen.

Hoofdstuk 7, Is het private spenderen aan R\&D gevoelig aan stijgingen van de fiscale incentives? Het Bewijs van het superbelastingsaftrekprogramma in Québec, onderzoekt de gevoeligheid van private uitgaven voor R\&D aan verhogingen van fiscale programma's ter ondersteuning van R\&D. Het bepaalde specifiek of bedrijven die een supergrote belastingsaftrek genoten, de belastingswinst van dit nieuw hulpmiddel vertaald hebben in meer uitgaven voor R\&D. In andere woorden werd de hoofdvraag besproken of de vermindering van de marginale kosten van R\&D ten gevolge van het superbelastingsaftrekprogramma in Québec de bedrijven die al genoten van een belastingsvoordeel, ertoe hebben aangezet hun inspanningen op het vlak van $R \& D$ te verhogen. De verkregen resultaten vertonen geen bewijs dat de bedrijven die al wat fiscale tegemoetkomingen genieten hun investeringen in R\&D verhogen als de belastingsvoordelen aanzienlijk verhoogd worden. Dus is er een zekere drempel achter dewelke overheidssteun minder doeltreffend wordt. In overeenstemming met daarmee mag het crowding-out-fenomeen in het geval van de superbelastingsaftrek in Québec niet volledig uitgesloten worden en dat vooral omdat de fiscale tegemoetkomingen gebaseerd zijn op het niveau.

In hoofdstuk 8, De impact van overheidsfinanciering op $R \& D$, innovatie en productiviteit. Resultaten van de microdata-enqûete in Québec, wordt de impact geanalyseerd van overheidsfinanciering op de innovatie-output en op de arbeidsproductiviteit door middel van gegevens van producerende bedrijven in Québec van 2002 tot 2004 volgens een model waarbij de overheidsincentives de winst van de innovatie verhogen. Bouwend op zijn vermogen om de intensiteit van de overheidssteun, de selectiviteit en de potentiële simultane standaardafwijking te controleren voegt het model toe aan het bewijs dat gesponsorde bedrijven beter presteren dan niet-gesponsorde bedrijven. In het bijzonder wordt een stijging van 1 $\%$ van de overheidssteun geassocieerd met een hogere waarschijnlijkheid van 0,32 $\%$ om innovatief te zijn, wat dus spreekt voor de tussenkomst van de regering. Bovendien rapporteren de resultaten van de schattingen een hoge elasticiteit van de innovatieve verkoop met betrekking tot de uitgaven voor innovatie van 0,83 voor gesponsorde bedrijven en een arbeidsproductiviteitselasticiteit met betrekking tot de innovatieve verkoop van het bedrijf per werknemer van 0,37 . Wat de impact van overheidsfinanciering betreft, tonen de resultaten dat een verhoging van de overheidssteun met $1 \%$ geassocieerd wordt met een verhoging in de uitgaven voor innovatie van 0,99 \%, d.w.z. bijna $1 \%$. Aan de andere kant doet een stijging van de overheidssteun van $1 \%$ de innovatieve verkoop stijgen met $0,82 \%$ en de arbeidsproductiviteit van het bedrijf met $0,31 \%$. Bovendien worden de resultaten 
van Québecs producerende bedrijven vergeleken met die van 18 landen, al dan niet leden van de OESO, waarbij een model wordt gebruikt dat ontwikkeld werd door een team van de OESO als deel van een microdataproject. Uit de resultaten blijkt dat producerende bedrijven in Québec die investeren in innovatie een prestatie op het vlak van de arbeidsproductiviteit kunnen voorleggen die vergelijkbaar is met die van de OESO-landen. De aanbeveling voor het beleid die volgt uit die resultaten is dat de regering van Québec bij zijn inspanningen om de productiviteitskloof te verkleinen een actiever en specifieker beleid op het vlak van de ondersteuning van $R \& D$ en innovatie moet aannemen om zich specifieker te richten op de industrieën en sectoren waar er tussenkomst van de overheid nodig is.

Hoofdstuk 9, Conclusies, presenteert de voornaamste bevindingen voor de specifieke behandelde onderzoeksvragen. Daarna worden er beleidsimplicaties en aanbevelingen gepresenteerd. Ten slotte worden de beperkingen benadrukt en wordt er een kader getekend voor toekomstig onderzoek. 


\section{Summary in French (Resumé)}




\section{Impacts des incitatifs gouvernementaux à la R-D, l'innovation et la productivité: \\ Une analyse microéconométrique du cas du Québec}

Le Chapitre 1, General introduction, a introduit l'objet de la thèse et a présenté sa pertinence et les questions de recherche abordées.

Depuis longtemps, le Québec offre aux entreprises de généreux incitatifs directs et indirects à la R-D et à l'innovation qui, dans le cas des incitatifs indirects, sont parmi les plus importants au monde. Toutefois, il n'est pas certain que ces encouragements gouvernementaux atteignent leur objectif, c'est-à-dire, permettre une augmentation significative de l'investissement privé de R-D capable de stimuler l'innovation et la productivité du travail. Cela est d'autant moins clair qu'en dépit de ces généreux incitatifs, le Québec accuse toujours un retard comparativement à ses voisins à l'égard de la productivité. Dans la littérature économétrique, il n'y a pratiquement pas de travaux publiés concernant l'évaluation de l'efficacité de ces mesures incitatives du gouvernement. Cette étude visait à combler ce vide en évaluant de manière empirique l'efficacité des mesures directes et indirectes de soutien du gouvernement à la R-D et l'innovation sur l'investissement privé de R-D des entreprises et sur leur performance économique, en particulier en termes d'innovation et de productivité du travail. Pour aborder ces problèmes, des questions de recherche spécifiques ont été formulées sur la base des données disponibles. Ces questions, qui ensemble forment cette thèse, étaient les suivantes :

1) Une politique fiscale qui consiste à subventionner les traitements et salaires de chercheurs se traduit-elle par un taux accru de chercheurs? (Chapitre 3)

2) Les incitatifs fiscaux à la R-D (incitations indirectes) au Québec sont-ils efficaces? En particulier, de combien l'investissement privé de R-D augmente-t-il pour un manque à gagner d'un dollar de recettes fiscales de R-D? (Chapitre 5)

3) Les incitatifs fiscaux en volume sont-ils plus efficaces que les incitatifs fiscaux incrémentaux? (Chapitre 5)

4) L'investissement privé de R-D est-il sensible aux incréments dans les incitations fiscales? (Chapitre 7)

5) Les subventions publiques directes augmentent-elles l'investissement privé de R-D? Quel est l'impact de ces incitatifs sur la productivité des entreprises? (Chapitre 6)

6) Les combinaisons des méthodes d'intervention publique actuellement utilisées au Québec pour encourager la R-D sont-elles optimales? (Chapitre 6) 
7) Quel est l'impact de l'ensemble des incitatifs publics (directs et indirects) sur la R-D des entreprises, l'innovation et la productivité au Québec? Comment se comparent les entreprises du Québec aux entreprises d'autres juridictions? (Chapitre 8)

8) Peut-on attribuer les causes du retard de la productivité du travail du Québec à une sous-performance des entreprises? (Chapitres 3 et 8)

Le Chapitre 2, Overview, définit les concepts clés tels que " recherche et développement expérimental » au sens de la législation applicable au Québec. II a également abordé la justification de l'appui du gouvernement à la R-D et l'innovation et, enfin, il a présenté l'environnement administratif provincial et fédéral pour le financement de la R-D et l'innovation.

Le Chapitre 3, R\&D and Innovation Policies in Québec, a donné un aperçu de la situation du Québec en ce qui concerne la R-D et l'innovation. Il a d'abord présenté un aperçu historique des politiques de R-D et d'innovation menées au Québec. Il a ensuite présenté un examen approfondi des incitatifs directs et indirects, y compris leurs coûts, offerts aux entreprises performant la R-D et aux innovateurs situés au Québec par le gouvernement fédéral et le gouvernement du Québec. Cette présentation est complétée par une comparaison interprovinciale des incitatifs à la R-D offerts aux entreprises. Ensuite, il a présenté les statistiques sur la R-D et l'innovation au Québec, en particulier, celles liées aux investissements publics et privés à la R-D et à l'innovation. En outre, il a présenté une comparaison interprovinciale et à l'échelle internationale afin de situer le Québec dans le monde. Enfin, il a discuté l'intensité de R-D et la question du retard de productivité à la lumière des statistiques présentées.

Le Chapitre 4, Data sources and construction of datasets, décrit les sources des bases de données et la construction des données utilisées dans l'analyse empirique. L'approche utilisée et les obstacles rencontrés dans le processus de jumelage des fichiers provenant d'enquêtes de Statistique Canada et des données administratives de Revenu Québec. II a ensuite présenté les résultats du processus de jumelage entre les fichiers de données.

Le Chapitre 5, Effectiveness of R\&D Tax incentives in manufacturing sector in Québec, a traité de l'évaluation de l'efficacité des incitatifs fiscaux à la R-D au Québec, à l'aide de données d'entreprises manufacturières de 1997 à 2003 provenant d'enquêtes de R-D, d'enquêtes annuelles sur les manufactures et de données administratives. L'élasticité-prix de la R-D estimée est de -0,10 à court terme et de -0,14 à long terme, avec une élasticité légèrement plus élevée pour les petites entreprises que pour les grandes entreprises. Nous avons montré qu'il y a 
une perte sèche liée aux incitatifs fiscaux à la R-D basés sur le volume et celle-ci est particulièrement accentuée pour les grandes entreprises. Pour les petites entreprises, elle n'est pas suffisante pour supprimer l'additionnalité de la R-D, du moins, pas pendant un certain nombre d'années après le changement initial à la mesure fiscale. Les crédits d'impôt incrémentaux à la R-D ne souffrent pas d'une telle perte sèche et sont, de ce point de vue, préférable aux incitatifs fiscaux basés sur le volume. Sur la base de ces résultats, nous avons recommandé que, si elle veut mener une politique fiscale de soutien à la R-D efficiente, la province du Québec devrait continuer à utiliser à la fois le crédit d'impôt incrémental et les crédits d'impôt basés sur le volume en faveur des petites entreprises. C'est précisément les petites entreprises qui ont des difficultés à obtenir du financement extérieur pour leur effort de R-D. Enfin, nous avons suggéré qu'il serait intéressant dans les travaux futurs de comparer les rendements sociaux de la R-D supportée par le gouvernement par le biais des crédits d'impôt et des subventions directes, sur la R-D supportée et non supportée par le gouvernement, et sur le soutien à la R-D pour les grandes et petites entreprises.

Le Chapitre 6, Public R\&D Subsidies and Productivity Evidence from firmlevel data in Québec, a analysé empiriquement les effets des subventions publiques directes à la R-D sur l'investissement privé de R-D et sur la croissance de la productivité des entreprises manufacturières dans un contexte où les incitatifs fiscaux sont présents. En utilisant l'estimateur semi-paramétrique conditionnel de différence-en-différences sur des données longitudinales de firmes québécoises, nous avons montré que les entreprises qui utilisent des subventions directes à la R-D avec les crédits d'impôt à la R-D obtiennent de meilleurs résultats en termes d'additionnalité de la R-D que les entreprises qui n'utilisent que les crédits d'impôt à la R-D. Nous avons ensuite utilisé une fonction de production pour évaluer l'efficacité des subventions publiques à la R-D sur la croissance de la productivité des entreprises. En particulier, nous avons trouvé dans la première étape de l'étude que l'intensité de R-D des entreprises dans le premier groupe est supérieure à celle des entreprises dans le dernier groupe. Dans la deuxième étape de l'étude, le premier résultat principal était que le rendement des dépenses de R-D financées par les subventions publiques à la R-D est de 0,134 , ce qui veut dire que pour chaque dollar supplémentaire de R-D de subvention publique, la production augmente de 0,134 dollar. Le deuxième résultat principal était que les subventions publiques à la R-D représentent $42 \%$ de la productivité induite par l'investissement privé et par les crédits d'impôt à la R-D. Ce résultat appui l'utilisation des subventions publiques à la R-D comme instrument de politique supplémentaire aux crédits d'impôt. 
Le Chapitre 7, Is Private R\&D Spending Sensitive to Increments in Fiscal incentives? Evidence from the Superdeductions Program in Québec, a examiné la sensibilité des dépenses privées de R-D aux augmentations des encouragements fiscaux à la R-D. Spécifiquement, il a déterminé si les entreprises recevant les superdeductions ont transferé sur leurs dépenses de R-D le gain fiscal additionnel provenant de ce programme d'aide. En d'autres termes, la question principale était de savoir si la réduction du coût marginal de la R-D entraînée par le programme de superdeductions au Québec avait conduit les entreprises bénéficiant déjà d'un avantage fiscal à augmenter leur effort de R-D. Les résultats obtenus ne montrent pas d'évidence que les entreprises qui reçoivent déjà un soutien fiscal à la R-D augmentent leur investissement en R-D dans une situation où l'aide fiscale est significativement incrémentée. Ainsi, il y a un certain seuil au-delà duquel le financement du gouvernement devient moins efficace. En conséquence, le phénomène d'éviction ne peut pas être totalement exclu dans le cas du programme québécois de superdeductions, du moins à court terme, et c'est principalement parce que l'aide fiscale est basée sur le volume des dépenses de R-D.

Le Chapitre 8, Impact of government funding on R\&D, innovation, and productivity Results from the innovation survey microdata in Québec, a analysé les impacts du financement du gouvernement sur l'output d'innovation et la productivité du travail en appliquant, sur des données provenant d'entreprises manufacturières du Québec de 2002 à 2004, un modèle dans lequel les incitatifs gouvernementaux peuvent augmenter les profits des firmes innovatrices. Grâce à sa capacité de prendre en compte l'intensité du soutien gouvernemental, la sélectivité et le potentiel biais de simultanéité, le modèle économétrique utilisé permet de montrer que les entreprises bénéficiaires des incitatifs gouvernementaux ont une meilleure performance que les entreprises non bénéficiaires. Chez ces entreprises, les estimations montrent une élasticité élevée de 0,83 des ventes d'innovation par rapport à l'input, constitué notamment de dépenses de R-D induites par les incitatifs gouvernementaux. De plus, l'élasticité de la productivité du travail estimée par rapport aux ventes d'innovation est de 0,37. Cette élasticité est plus élevée pour les entreprises de haute et moyenne-haute technologie que pour les entreprises de moyenne-basse et basse technologie. En ce qui concerne l'impact du soutien gouvernemental, les résultats montrent qu'une augmentation de $1 \%$ de ce dernièr est associée à une croissance de 0,99\%, donc presque $1 \%$, des dépenses privées d'innovation. D'autre part, une augmentation de $1 \%$ du soutien gouvernemental est liée à une augmentation des ventes d'innovation de $0,82 \%$ et de la productivité du travail des firmes de 0,31\%. Par ailleurs, les entreprises manufacturières du Québec ont été comparés à ceux de 18 pays membres et non membres de I'OCDE en utilisant un modèle développé par une équipe de l'OCDE dans le cadre d'un projet de microdonnées. Les résultats montrent que les entreprises manufacturières 
du Québec qui investissent en innovation ont une performance en matière de productivité du travail comparable à celle des pays de l'OCDE. La recommandation de politique découlant de ces résultats est que, dans son effort pour réduire l'écart de productivité, le gouvernement du Québec devrait adopter des politiques de soutien à la R-D et à l'innovation plus actives et spécifiques afin de cibler plus spécifiquement les industries ou les secteurs nécessitant une intervention gouvernementale.

Le Chapitre 9, Conclusions, a présenté les principales conclusions pour les questions de recherche spécifiques abordées. II a ensuite présenté des implications politiques et des recommandations. Enfin, il a souligné les limites et a tracé quelques avenues pour la recherche future. 




\section{Curriculum Vitae}

Rufin Sosthène Arsene Baghana was born on April 16, 1971 in Brazzaville, Congo. He studied Economics at the École des Science de la Gestion (ESG), Université du Québec à Montréal (Canada), where he graduated with a Master's degree. He also followed part of his doctoral studies at the ESG. During his academic career, Rufin held teaching positions for two consecutive years (2000-2001). He acted as teaching assistant for an Econometric course for the MBA program at the ESG. In 2001, Rufin joined the Ministère des Finances du Québec, where he currently works as an advisor in the Financial Organisation and State-Owned Enterprises division. 


\section{UNITED NATIONS UNIVERSITY}

\section{UNU-MERIT}

2013

\section{Rufin Baghana}

Impacts of Government Incentives to R\&D, Innovation and Productivity:

A Microeconometric Analysis of the Québec Case

\section{Lilia I. Stubrin}

High-Tech Activities in Emerging Countries: A Network perspective on the Argentinean biotech activity

\section{2}

\section{Abdul Waheed}

Innovation Determinants

and Innovation as a Determinant:

Evidence from Developing Countries

\section{Bilal Mirza}

Energy Poverty and Rural Energy Markets in Pakistan

\section{Benjamin Engelstätter}

Enterprise Software and Video Games: An

Empirical Analysis

\section{Fulvia Farinelli}

Natural Resources, Innovation and Export Growth: The Wine Industry in Chili and Argentina

\section{Rodolfo Lauterbach}

Innovation in Manufacturing: From Product Variety and Labor Productivity Growth to Economic Development in Chile

\section{Kirsten Wiebe}

Quantitative Assessment of Sustainable Development and Growth in Sub-Saharan Africa.

\section{Julio Miguel Rosa}

Organizational Strategies, Firms' Performance and Spatial Spillovers. The Canadian Case in Research and Development.

\author{
Johannes Wilhelmus Marie Boels \\ Joseph Schumpeter, honderd jaar \\ economische ontwikkeling. Een historisch- \\ theoretische beschouwing.
}

2011

\section{Daniel Vertesy}

Interrupted Innovation: Emerging economies in the structure of the global aerospace industry.

\section{Tina Saebi}

Successfully managing alliance portfolios: an alliance capability view.

\section{Nora Engel}

Tuberculosis in India - A case of innovation and control.

\section{Evans Mupela}

Connectivity and growth in Sub-Saharan Africa: The role of communication satellites

\section{Nantawan Kwanjai}

Cross cultural intelligence amid intricate cultural webs - A tale of the UnDutchables in the land of 1002 smiles

\section{Lina Sonne}

Innovation in Finance to Finance Innovation: Supporting pro-poor entrepreneur-based innovation

2010

\section{Fernando Santiago}

Human Resources Management Practices and Learning for Innovation in Developing Countries: Pharmaceutical Firms in Mexico

\section{Zakaria Babutsidze}

Essays on Economies with Heterogenous Interacting Consumers

\section{Bertha Vallejo}

Learning and Innovation Under Changing Market Conditions: The Auto Parts Industry in Mexico

\section{Donatus Ayitey}

Technical Change, Competitiveness and Poverty Reduction: A Study of the Ghanaian Apparel Industry

\section{Sergey Fillipov}

Multinational Subsidiary Evolution: Corporate Change in New EU Member States 


\section{Asel Doranova}

Technology Transfer and Learning under the Kyoto regime; Exploring the Technological Impact of CDM projects in developing countries

\section{9}

\section{Alexis Habiyaremye}

From Primary Commodity Dependence to Diversification and Growth". "Absorptive Capacity and Technological Catch Up in Botswana and Mauritius".

\section{Yoseph Getachew}

The Role of Public Capital in Economic Development

\section{Sandra Leitner}

Embodied Technological Change and Patterns of Investment in Austrian Manufacturing

\section{Semih Akçomak}

The Impact of Social Capital on Economic and Social Outcomes

\section{Abraham Garcia}

The Role of Demand in Technical Change

\section{Saurabh Arora}

Coherence in socio-technical systems: a network perspective on the innovation process

\section{8}

\section{Rutger Daems}

Medicines for the developing world

\section{Johannes Hanel}

Assessing Induced Technology - Sombart's Understanding of Technical Change in the History of Economics

\section{Rifka Weehuizen}

Mental Capital: the economic significance of mental health

\section{Danielle Cloodt}

The relationship between R\&D partnership formation, social embeddedness and innovative performance

\section{Sabine Fuss}

Sustainable Energy Development under Uncertainty
2007

\section{Tobias Kronenberg}

Reconciling Environmental Conservation with Economic Prosperity: The Feasibility of Double Dividends in the Short and Long Run

\section{Viktoria Kravtsova}

Assessing the Impact of Foreign Direct Investment in Transition Economies

\section{Suhail Sultan}

The Competitive Advantage of Small and Medium Sized Enterprises: The Case of Jordan's Natural Stone Industry

2006

\section{Bulat Sanditov}

Essays on Social Learning and Imitation

\section{Mamata Parhi}

Dynamics of New Technology Diffusion: A Study of the Indian Automotive Industry

\section{Andreas Reinstaller}

Social structures and the innovation process:

Their role in the demand of firms and consumers

\section{Rose Kiggundu}

Innovation systems and development : the journey of a Beleaguered Nile Perch Fishery in Uganda

\section{Thomas Pogue}

The Evolution of Research Collaboration in South African Gold Mining: 1886-1933

\section{Geoffrey Gachino}

Foreign Direct Investment, Spillovers and Innovation: The Case of Kenyan Manufacturing Industry

\section{0. Önder Nomaler}

Technological Change, International Trade and Growth - An Evolutionary, Multi-Agents-Based Modeling Approach

2005

\section{Samia Satti Osman Mohamed-Nour}

Change and Skill Development in the Arab Gulf Countries

\section{Elad Harison}

Intellectual Property Rights: Economics and

Policy Analysis 


\section{Daniel Dalohoun}

The relationship between R\&D partnership formation, social embeddedness and innovative performance: a multi-level approach of social embeddedness

\section{Müge Ozman}

Networks, Organizations and Knowledge

\section{Bas Straathof}

Product variety and economic growth - The counteracting effects of scale and idiosyncrasy

\section{Wilfred Schoenmakers}

Knowledge Flows between Multinational Companies: A Patent Data Analysis

\section{Myriam Cloodt}

Mergers and Acquisitions (M\&As) in High-Tech Industries: Measuring the Post-M\&A

Innovative Performance of Companies

\section{4}

\section{Paola Criscuolo}

R\&D Internationalisation and Knowledge Transfer. Impact on MNEs and their Home Countries

\section{Maarten Verkerk}

Trust and Power on the Shop Floor

\section{Gottfried Leibbrandt}

Adoption, harmonization and succession of network technologies across countries

\section{Mark Sanders}

Skill Biased Technical change - Its Origins, the Interaction with the Labour Market and Policy Implications

\section{3}

\section{Nadine Roijakkers}

Inter-firm cooperation in high-tech industries: a study of R\&D partnerships in pharmaceutical biotechnology

\section{Viki Sonntag}

Speed, Scale and Sustainability

\section{Masaru Yarime}

From End-of-Pipe Technology to Clean

Technology

\section{Stéphane Malo}

The combinatorial Chemistry Revolution Sustaining a Superior Performance Position through Technological Learning
2002

\section{Annelies Hogenbirk}

Determinants of Inward Foreign Direct

Investment: the Case of the Netherlands

2001

\section{John Adeoti}

Technology Investment in Pollution Control in Sub-Saharan Africa: The Case of the Nigerian Manufacturing Industry

\section{Edward Huizenga}

Innovation Management: How Frontrunners Stay Ahead. An Empirical Study on Key Success Factors in the ICT sector

2000

\section{Machiel van Dijk}

Technological Change and the Dynamics of Industries. Theoretical Issues and Empirical evidence from Dutch Manufacturing

1999

\section{Jan Cobbenhagen}

Managing Innovation at the Company Level: A Study on Non-Sector-Specific Success Factors

\section{Marjolein Caniëls}

Regional Growth Differentials: The Impact of Locally Bounded Knowledge Spillovers

\section{8}

\section{Aldo Geuna}

Resource allocation and knowledge production: Studies in the economics of university research

\section{6}

\section{Reinoud Joosten}

Dynamics, Equilibria, and Values

\section{Hugo Kruiniger}

Investment, R\&D, and the Financing Decisions of the Firm 


\section{Hans van Meijl}

Endogenous Technological Change: The Case of Information Technology. Theoretical

Considerations and Empirical Results

\section{René Kemp}

Environmental Policy and Technical Change. A Comparison of the Technological Impact of Policy Instruments

\section{Rohini Acharya}

The Impact of New Technologies on Economic Growth and Trade. A Case Study of Biotechnology

\section{Geert Duysters}

The Evolution of Complex Industrial Systems.

The Dynamics of Major IT Sectors

\section{Marjan Groen}

Technology, Work and Organisation, A Study of the Nursing Process in Intensive Care Units

\section{4}

\section{Huub Meijers}

On the Diffusion of Technologies in a Vintage Framework; Theoretical Considerations and Empirical Results

\section{Theon van Dijk}

The Limits of Patent Protection. Essays on the Economics of Intellectual Property Rights

\section{Hans Voordijk}

Naar Integrale Logistiek in Bedrijfsketens, Ontwikkelingen in de Bouw

\section{3}

\section{Paul Diederen}

Technological Progress in Enterprises and Diffusion of Innovations. Theoretical Reflections and Empirical Evidence.

\section{Ben Dankbaar}

Economic Crisis and Institutional Change. The crisis of Fordism from the perspective of the automobile industry

\section{Hanno Roberts}

Accountability and Responsibility: The Influence of Organisation Design on

Management Accounting

\section{Bart Verspagen}

Uneven Growth Between Interdependent

Economies. An Evolutionary View on

Technology Gaps, Trade and Growth

\section{Sjoerd Romme}

A Self-organization Perspective on Strategy Formation

1989

\section{John Spangenberg}

Economies of Scale, and Atmosphere in Research Organisations

1988

\section{John Hagedoorn}

Evolutionary and heterodox innovation analysis : a study of industrial and technological development in process control and information technology 